\title{
Transcriptional activity of Npas4l controls endothelial lineage specification and restriction in zebrafish
}

\author{
Dissertation \\ zur Erlangung des Doktorgrades \\ der Naturwissenschaften \\ vorgelegt beim Fachbereich 15 \\ der Goethe-Universität \\ in Frankfurt am Main von
}

Kenny Mattonet

aus Berlin, Deutschland

Frankfurt 2021

(D30)
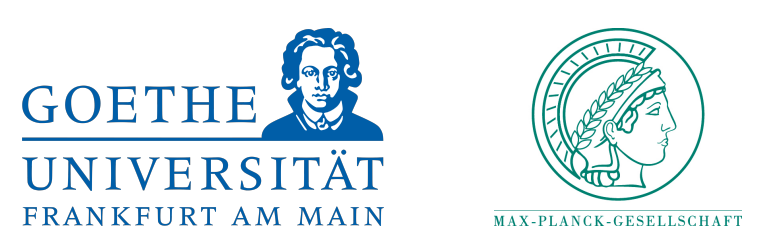

Max Planck Institute for Heart and Lung Research for Heart and Lung Rese
W.G. Kerckhoff Institute 



\section{vom Fachbereich Biowissenschaften (FB15) der \\ Johann Wolfgang Goethe - Universität als Dissertation angenommen.}

Dekan: Prof. Dr. Sven Klimpel

Gutachter:

Prof. Dr. Didier Y. R. Stainier

Prof. Dr. Ingo Ebersberger

Datum der Disputation: 



\section{Dem Andenken meiner Oma Marianne Glas (25.5.1929 - 10.12.2020) gewidmet.}

Du hast mich oft daran erinnert, wie ich dir als Kind versprochen habe, Krebs und Diabetes zu heilen.

Ich habe es nicht vergessen, es ist nur viel schwieriger als ich mir das damals vorstellen konnte. 



\section{Contents}

$\begin{array}{ll}\text { Deutsche Zusammenfassung } & 1\end{array}$

$\begin{array}{ll}\text { English summary } & 7\end{array}$

$\begin{array}{ll}\text { Populärwissenschaftliche Zusammenfassung } & 13\end{array}$

$\begin{array}{ll}\text { Popular science summary } & 15\end{array}$

1 Introduction $\quad 17$

1.1 Evolution of the vascular system . . . . . . . . . . . . 17

1.2 A cross-section through vascular develop-

ment in zebrafish . . . . . . . . . . . . . . . . 20

1.2.1 Endothelial cell origin . . . . . . . . . . . . 20

1.2.2 Specification of endothelial cells . . . . . . . . . 21

1.2.3 Endothelial migration and tubulogenesis . . . . . . . 23

1.2.4 Angiogenesis and ISV formation . . . . . . . . . . 24

1.2.5 Vascular maturation . . . . . . . . . . . . . 25

1.3 Transcription factors inducing endothelial specification . . . . 25

1.3.1 Npas4l .......................... 25

$1.3 .2 \quad$ Etsrp . . . . . . . . . . . . . . . . . . . . 29

1.3.3 Tall . . . . . . . . . . . . . . . 30

1.3.4 Lmo2 . . . . . . . . . . . . . . . . . 32

1.3.5 Other early endothelial factors . . . . . . . . . 32

2 Aims $\quad 35$

3 Materials and methods $\quad 37$

3.1 Materials . . . . . . . . . . . . . . . . . 37

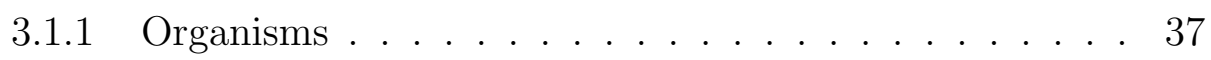

3.1.1.1 Bacterial strain . . . . . . . . . . 37

3.1.1.2 Established zebrafish lines used in this study . 37

3.1.1.3 Self-generated zebrafish lines . . . . . . . 38 
3.1.1.4 Zebrafish feeding . . . . . . . . . . . . 39

3.1.1.5 Laboratory equipment . . . . . . . . . . . 39

3.1.1.6 Laboratory consumables . . . . . . . . . . . . 42

3.1.2 Chemical reagents and macromolecules . . . . . . . . . 43

3.1.2.1 Antibiotics . . . . . . . . . . . . 43

3.1.2.2 Antibodies . . . . . . . . . . . . 43

3.1.2.3 Buffers. . . . . . . . . . . . . . . 44

3.1.2.4 Chemicals . . . . . . . . . . . . . . 44

3.1.2.5 Enzymes . . . . . . . . . . . . . 46

3.1.2.6 Growth media . . . . . . . . . . . . 46

3.1 .2 Kits . . . . . . . . . . . . . . 46

3.1.2.8 Oligonucleotides . . . . . . . . . . . 47

3.1.2.9 Plasmids ................ 53

3.1 .3 Software . . . . . . . . . . . . . . 54

3.1 .4 Databases . . . . . . . . . . . . . . 54

3.1.5 Services ..................... 55

3.2 Methods . . . . . . . . . . . . . . . . . 55

3.2.1 Genome editing using CRISPR/Cas9 technology . . . . 55

3.2.1.1 npas4l $l^{\text {Pt(npas } 4 l-V 5) b n s 314} \ldots \ldots \ldots \ldots 5$

3.2.1.2 npas4l $l^{\text {Pt(npas } 4 \text { l-p2A-Gal4-VP16)bns313 } \ldots \ldots . . . . ~} 56$

3.2.1.3 npas4l $l^{\left.\text {Pt(+36bp-npas } 4 l-p 2 A-G a l_{4}-V P 16\right) b n s 423} \ldots \ldots 56$

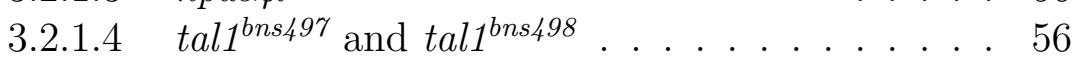

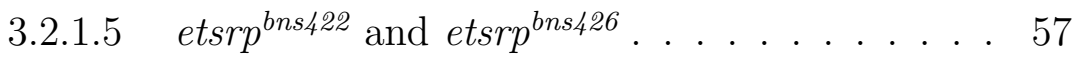

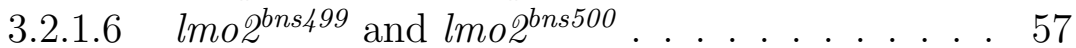

3.2.1.7 il11ra ${ }^{\text {Pt(il11ra-p2a-Gal4-VP16)bns424 } \ldots . . . . . . ~} 57$

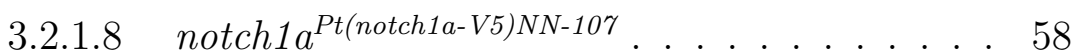

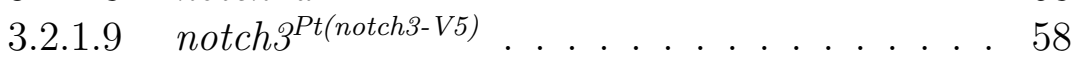

3.2.1.10 pdgfab bns322 (promoter deletion) . . . . . . . 59

3.2.1.11 pdgfba $a^{b n s 323} \ldots \ldots \ldots \ldots$. . . . . . . . . . 59

3.2.1.12 pdgfbb bns324 . . . . . . . . . . . . . 59

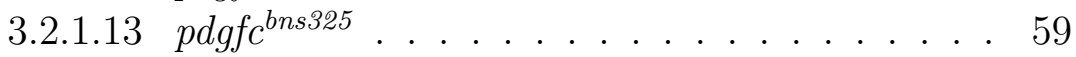

3.2.1.14 pdgfd bns326 . . . . . . . . . . . . 60

3.2.2 npas 4 - alleles and genotypes of the crosses used to generate the data . . . . . . . . . . . . 60

3.2.3 Zebrafish methods ............... 61

3.2.3.1 Zebrafish maintenance . . . . . . . . . . 61

3.2.3.2 Zebrafish breeding ............. . 61

3.2.3.3 Handling of zebrafish embryos . . . . . . . . . 62

3.2.3.4 Microinjections . . . . . . . . . . . . 62

3.2.3.5 Zebrafish screening . . . . . . . . . . . 63

3.2.3.6 Transplantation . . . . . . . . . . . . . 64 
3.2.3.7 Anesthesia of adult zebrafish . . . . . . . 65

3.2.3.8 Zebrafish genotyping . . . . . . . . . 65

3.2.3.9 Zebrafish killing. . . . . . . . . . . . . 66

3.2.4 Molecular biology . . . . . . . . . . . . . . 66

3.2.4.1 gDNA-extraction . . . . . . . . . . . . . 66

3.2.4.2 RNA-extraction . . . . . . . . . . . 66

3.2.4.3 DNA/RNA quantification and quality assessment . . . . . . . . . . . . 67

3.2.4.4 Agarose gel electrophoresis . . . . . . . . 68

3.2.4.5 cDNA first strand synthesis . . . . . . . . . 68

3.2.4.6 in vitro transcription . . . . . . . . . . . . . 68

3.2.4.7 Primer design . . . . . . . . . . . . . . . 69

3.2.4.8 Polymerase chain reaction (PCR) . . . . . . . 69

3.2.4.9 High resolution melt analysis (HRMA) . . . . 70

3.2.4.10 T7-endonuclease assay . . . . . . . . . . 70

3.2.4.11 Quantitative real-time PCR (qPCR) . . . . 70

3.2.4.12 Cloning of constructs using restriction enzymes 70

3.2.5 Histology and microsections . . . . . . . . . . . 71

3.2 .6 Immunohistochemistry . . . . . . . . . . . . . . 71

3.2 .7 in situ hybridization . . . . . . . . . . . . . 72

3.2.8 Transcriptional profiling . . . . . . . . . . . 73

3.2.8.1 Sample preparation and cell sorting . . . . . 73

3.2.8.2 Bulk RNAseq . . . . . . . . . . . . . . . . 73

3.2.8.3 Single cell RNAseq . . . . . . . . . . . . . . 74

3.2 .9 Imaging . . . . . . . . . . . . . . . . . . . . 74

3.2.9.1 Mounting . . . . . . . . . . . 74

3.2.9.2 Confocal and spinning disk imaging . . . . . 75

3.2.9.3 Lightsheet imaging . . . . . . . . . . . . . . 75

3.2.10 Image processing and analysis . . . . . . . . . . 76

3.2.11 Quantification of ISV numbers . . . . . . . . . . . 76

3.2.12 Statistical analyses . . . . . . . . . . . . 76

4 Results $\quad 77$

4.1 Targeting and knock-in strategies . . . . . . . . . . . . 77

4.2 Generation of the npas4l-V5bns314 allele . . . . . . . . . . 81

4.3 Npas4l-V5 is detectable on the protein level during early embryonic development . . . . . . . . . . . . . . 83

4.4 Generation of the npas $4 l^{\text {bns313 }}$ reporter allele . . . . . . . . . 85

4.5 npas 4 l reporter positive cells can be detected in endothelial cells from early somite stages onwards . . . . . . . . 86

4.6 npas4l reporter expression in adult zebrafish . . . . . . . . . 90 
4.7 The npas $4 \mathrm{l}$ reporter is re-expressed during regenerative processes 93

4.8 Generation of a UAS:mTagBFP reporter line for multi-color imaging of Gal4-reporters . . . . . . . . . . . . . . . 95

4.9 Generation of a mutated version of the npas4l reporter lacking Npas4l function . . . . . . . . . . . 97

4.10 Characterization of the mutated npas $4 l^{\text {bns } 423}$ reporter allele . . . . . . . . . . . . 100

4.11 Bulk transcriptomic profiling of the npas 4 l reporter positive lineage in npas $4 l$ heterozygous and mutant embryos . . . . . . 105

4.12 Single cell transcriptomic profiling of the npas $4 l$ positive lineage in npas4l heterozygous and mutant embryos . . . . . . 108

4.13 Generation of out-of-frame and in-frame mutants for etsrp, tal1 and lmo2 . . . . . . . . . . . . . 114

4.14 etsrp, tal1 and lmo2 mutants show distinct phenotypes . . . . 115

4.15 npas $_{4} \mathrm{l}^{-} ; \mathrm{etsrp}^{-/}$and tal1-/- etsrp $^{-/}$double mutants lack endothelial cells completely . . . . . . . . . . . . . . . . 121

4.16 tal1-mRNA overexpression rescues specification of endothelial cells in npas4l mutants . . . . . . . . . . . . . . . . . . . . . 122

4.17 npas 4 l-mRNA injection enhances endothelial transplantation efficiency . . . . . . . . . . . . . . . 127

\section{Discussion}

5.1 npas4l-V5ns314 enables Npas4l protein detection and retains Npas4l function . . . . . . . . . . . . . . . . . . 131

5.2 The npas4 $l^{\text {bns313 }}$ allele labels npas $4 l$ positive cells in vivo in embryos and adults . . . . . . . . . . . . . . 132

5.3 The npas $4 l$ reporter is reactivated during regenerative processes 133

5.4 The npas 4 l mutant phenotype is caused by multiple defects phenocopied by different downstream effectors . . . . . . . . 133

5.4.1 npas $4 \mathrm{l}$ reporter positive cells in npas $4 \mathrm{l}$ mutants do not migrate or differentiate and instead contribute to paraxial and intermediate mesoderm-derived tissues . . . . . 133

5.4.2 Tal1 and Lmo2 suppress an intermediate mesoderm fate134 5.4.3 Etsrp suppresses a paraxial mesoderm fate . . . . . . 135

5.5 Endothelial cells in zebrafish cannot form in absence of both Tall and Etsrp function . . . . . . . . . . . . 136

5.6 Future perspectives . . . . . . . . . . . . . . . . 137 5.6.1 What is the earliest defect in npas $4 \mathrm{l}$ mutants and when can it be observed for the first time? . . . . . . . . 137

5.6.2 A platform for the systematic testing of early transcription factors during vasculogenesis . . . . . . . . . 138 
5.6.3 npas4l function during heart development . . . . . . 139

5.6.4 Does tal1 overexpression in low doses cause somite defects? . . . . . . . . . . . . . . . . . . 139

5.6.5 Direct interaction partners of Npas4l . . . . . . . . . 140

5.6.6 How is etsrp expressed in the remaining endothelium of npas 4 l mutants? . . . . . . . . . . . . . . . . . . . 141

5.6.7 An upstream candidate of npas4l . . . . . . . . . . 142

5.6.8 The evolution of early endothelial transcription factors 143

5.6.9 npas4l reporter expression in species that lack endothelium or npas4l . . . . . . . . . . . . . . . 143

5.6.10 Rescuing npas4l mutants to adulthood . . . . . . . . . 144

5.6.11 Can npas4l overexpression be harnessed as a tool to improve endothelial transplantation efficiency? . . . . . 145

5.6.12 Miscellaneous experiments . . . . . . . . . . . . 145

5.6.12.1 Do npas $4 l$ mutants have an increased number of npas $4 l$ reporter positive myocardial cells? . 146

5.6.12.2 Is the npas4l reporter expressed in lymphangioblasts? . . . . . . . . . . . . 146

5.6.12.3 Is there a common progenitor of endothelial and blood cells?. . . . . . . . . . 146

5.6.12.4 Is the fli1a:GFP transgene transiently present in endothelial progenitors of npas 4 l mutants? 146

5.6.12.5 Does the npas4l reporter label all cardiomyocytes in the first heart field? . . . . . . . 147

\section{Appendix}

I List of abbreviations . . . . . . . . . . . . . . . . . 151

II Acknowledgements . . . . . . . . . . . . . . . . . . . . 153

III curriculum vitae. . . . . . . . . . . . . . . . . . . . . 155 



\section{Deutsche Zusammenfassung}

\section{Einleitung}

Die Bildung von Blutgefäßen ist essentiell für die Entwicklung und Homöostase von Wirbeltieren und die Endothelzellspezifikation ist ein wichtiger erster Schritt in diesem Prozess. Unser Wissen über die Endothelzellspezifikation stammt größtenteils aus Studien an Mäusen und Zebrafischen. Da sich Zebrafische in transparenten Eiern entwickeln und resistent gegen kardiovaskuläre Defekte in der frühen Entwicklung sind, stellen sie das ideale Modellsystem für die Untersuchung des Endothelzellverhaltens während der frühen Gefäßentwicklung dar (Stainier et al., 1996; Isogai et al., 2001).

Das früheste bekannte Ereignis bei der Endothelzellspezifikation im Zebrafisch ist die Expression des bHLH-PAS-Transkriptionsfaktor-Gens npas $4 l$ (Reischauer et al., 2016). cloche/npas4l-Mutanten fehlen die meisten Endothel- und Blutzellen (Stainier et al., 1995; Liao et al., 1997; Parker and Stainier, 1999). Mechanistisch reguliert Npas4l die Expression der Transkriptionsfaktorgene etsrp, tal1 und lmo2 (Qian et al., 2005; Marass et al., 2019), wodurch die Bildung von Endothel- und Blutzellen gefördert wird. Die Vorläuferzellen bilden sich im Seitenplattenmesoderm und bewegen sich entlang der ventralen Seite der Somiten in Richtung Mittelachse, wo sie zu einem Gefäßstrang zusammenwachsen, der die beiden großen axialen Gefäße bildet: die dorsale Aorta und die hintere Kardinalvene (Jin et al., 2005; Helker et al., 2015).

Etsrp ist ein Mitglied der ETS-Transkriptionsfaktorfamilie, das für die Spezifikation von Endothel und myeloiden Zellen erforderlich ist (Sumanas und Lin, 2006; Pham et al., 2007). In etsrp-Mutanten und -Morphanten im Zebrafisch erwerben Endothelvorläuferzellen alternative Schicksale, einschließlich dem von Herz- und Skelettmuskelzellen, was zu einem Mangel an Endothelzellen führt (Palencia-Desai et al., 2011; Chestnut et al., 2020). Globale etsrp-Überexpression in Zebrafischembryonen führt zu einem Anstieg 
der Expression von Endothelmarkern (Kuan et al., 2009; Veldman et al., 2013).

TAL1 ist ein bHLH-Transkriptionsfaktor, der für die Entwicklung von Endothelzellen und Blut benötigt wird. In Mäusen, denen Tal1 fehlt, sind sowohl der Gefäßplexusumbau als auch die hämatopoetische Entwicklung beeinträchtigt (Faloon et al., 2000; Robertson et al., 2000). In ähnlicher Weise fehlen bei tal1-Zebrafischmutanten die Expression des erythroiden Markers gata1a sowie Erythrozyten (Liao et al., 1998). Außerdem weisen tal1-Mutanten vaskuläre Defekte auf (Bussmann et al., 2007). Die globale Überexpression von tal1 in npas4l-Mutanten führt zu einer teilweisen Rettung der Endothel- und Blutzelldifferenzierung (Liao et al., 1998).

LMO2 ist ein LIM-Domänen enthaltender Transkriptionsfaktor, der für die hämatopoetische Entwicklung benötigt wird. LMO2-Funktionsverlustmodelle in Mäusen wie auch Zebrafischen zeigen eine defekte erythroide Entwicklung und Markergenexpression (Warren et al., 1994; Patterson et al., 2007). lmo2Zebrafischmutan-ten haben auch vaskuläre Defekte, die wahrscheinlich durch reduzierte Endothelzellmigration hervorgerufen werden (Weiss et al., 2012; Matrone et al., 2017). LMO2 ist an der Bildung von Transkriptionsfaktorkomplexen beteiligt, die unter anderem TAL1 enthalten(Warren et al., 1994; Osada et al., 1995), und es wurde berichtet, dass LMO2 als Teil eines Gerüsts für Transkriptionsfaktorkomplexe funktioniert (El Omari et al., 2011).

\section{Zwei Knock-in-Reporterlinien für npas $4 l$ ermöglichen die Visualisierung der npas4l-Expression und den Nach- weis des Npas4l-Proteins}

Ich habe eine transgene V5-Linie zum Nachweis des markierten Npas4l auf Proteinebene und eine Gal4-VP16-Reporterlinie zur Visualisierung und Verfolgung von npas4l exprimierenden Zellen in vivo generiert. Beide Linien können bereits in frühen Entwicklungsstadien nachgewiesen werden und werden unter anderem in Endothelvorläuferzellen exprimiert. Während das Npas4l-V5-Signal schwach und transient in den Zellkernen früher Endothelvorläuferzellen detektiert wird, zeigt die Gal4-Reporterlinie eine starke und stabile Expression, die eine Verfolgung von npas 4l-Reporter-positiven Zellen während der gesamten Embryonal- und frühen Larvenentwicklung ermöglicht. Beide Reporterlinien sind als homozygote Träger des jeweiligen Allels lebensfähig und komplementieren auch starke npas\&l-Mutantenallele, was darauf 
hindeutet, dass die endogene Npas4l-Funktion der Allele, die den Reporter tragen, nicht gestört ist.

\section{npas4l-Reporter exprimierende Zellen in npas $4 l \mathrm{Mu}-$ tanten wandern nicht zur Mittelachse und differenzieren zu Skelettmuskelzellen und Zellen der pronephrischen Tubuli}

Um npas4l-Reporter exprimierende Zellen in npas4l-Mutanten zu verfolgen, habe ich eine mutierte Variante der im vorigen Abschnitt beschriebenen Gal4Reporter-linie erzeugt. In dieser Mutante trägt die Region, die für die DNABindungsstelle von Npas4l kodiert, eine Insertion, die den Leserahmen nicht verschiebt. Dadurch stört sie die Npas4l-Funktion, aber nicht die Reporterexpression. Dieses mutierte Reporterallel komplementiert nicht die npas4lMutanten und zeigt einen starken Phänotyp, was darauf hindeutet, dass es sich um ein funktionelles Nullallel handelt.

Phänotypische Analysen zeigten, dass npas4l-Reporter exprimierende Zellen in npas4l-Mutanten nicht spezifizieren oder zur Mittelachse wandern. Stattdessen tragen sie zu den vom intermediären Mesoderm abgeleiteten pronephrischen Tubuli und dem vom paraxialen Mesoderm abgeleiteten Skelettmuskel bei. Ich habe diese Phänotypen durch Einzelzell-RNAseq an den npas4l-Reporter positiven Zellen in npas4l $l^{+/-}$und npas4t/- Embryonen bestätigt. Zusammen erklären diese beiden alternativen Zellschicksale den Großteil der beobachteten Veränderungen zwischen den Genotypen.

\section{Etsrp, Tal1 und Lmo2 regulieren verschiedene Aspekte der Endothelentwicklung}

Npas4l ist dafür bekannt, die Expression der drei Transkriptionsfaktorgene etsrp, tal1 und lmo2 (Marass et al., 2019) zu fördern. Ich stellte die Hypothese auf, dass das Fehlen jedes dieser Transkriptionsfaktoren in npas4lMutanten verschiedene Aspekte des npas4l-Phänotyps verursacht. Daher habe ich Mutantenlinien für alle drei Gene generiert und sie sowohl in vaskulären Reporterlinien als auch im npas4l-Reporterhintergrund analysiert. Diese neuen mutierten Allele rekapitulieren alle zuvor veröffentlichten Phänotypen, einschließlich der vaskulären Defekte und des Verlusts von roten Blutkörperchen in tal1- (Bussmann et al., 2007) und lmo2-Mutanten (Weiss et al., 2012). Ich beobachtete, dass Endothelvorläufer in etsrp-Mutanten in Richtung Mittelachse wandern, aber nicht differenzieren und keine Vaskulogenese 
durchlaufen. Einige von ihnen scheinen zu Skelettmuskelzellen zu differenzieren, was zuvor bereits bei etsrp-Mutanten beobachtet wurde (Chestnut et al., 2020). Im Gegensatz dazu zeigen tal1-Mutanten eine defekte Migration von npas4l-Reporter exprimierenden Zellen, aber keine Zunahme von npas 4l-Reporter exprimierenden Skelettmuskelzellen. Stattdessen differenzieren diese Zellen zu aus dem intermediären Mesoderm stammenden pronephrischen Tubuluszellen. Ich zählte die Anzahl der npas4l/Pax2a doppelt positiven Zellen in den pronephrischen Tubuli aller vier Mutanten und sah deren Zunahme in npas4l- und tal1-Mutanten, aber nicht in etsrp-oder lmo2Mutanten. Allerdings waren viele npas 4 -Reporter exprimierende Zellen in der hinteren Kardinalvene von lmo2-Mutanten auch Pax2a-positiv, was auf endotheliale Differenzierungsdefekte hinweist.

\section{fli1a positive Endothelzellen entwickeln sich nicht in etsrp $^{-/-} ;$tal1 $^{-/-}$oder etsrp ${ }^{-/-} ;$npas4 $\mathrm{l}^{/-}$Mutanten}

npas4l-, etsrp- und tal1-Mutanten zeigen alle schwere vaskuläre Phänotypen. Einige Endothelzellen und vaskuläre Strukturen bleiben jedoch in jeder Mutante erhalten. Der Phänotyp ist am stärksten in npas $4 l^{-/}$-Embryonen, aber selbst in diesen Embryonen können einige fli1a-positive Endothelzellen in der Schwanzregion beobachtet werden. Es war unklar, ob sich diese Population von Endothelzellen unabhängig von der Npas4l-, Tal1- und EtsrpFunktion entwickelt oder als Folge einer restlichen tal1- oder etsrp-Expression unabhängig von Npas4l. Um diese Frage zu untersuchen, habe ich Doppelmutanten generiert und nach dem Vorhandensein von fli1a-positiven Endothelzellen in diesen Mutanten gesucht. während fli1a-positive Endothelzellen in npas $4 t^{-/-}$- und npas4t $\mathrm{t}^{--} ;$tal1 $^{-/-}$-Embryonen deutlich vorhanden sind, können keine solchen Zellen in npas4t/--; etsrp $^{-/-}$- oder etsrp $^{-/-} ;$tal1 $^{-/-}$-Embryonen beobachtet werden. Diese Daten deuten darauf hin, dass sich im Zebrafisch keine Endothelzellen entwickeln können, wenn npas $4 l$ und etsrp oder etsrp und tal1 zugleich gestört sind. während der Verlust von etsrp zu stärkeren Defekten in npas 4l-Mutanten führt, gibt es keinen zusätzlichen Phänotyp, der durch den Verlust von tal1 verursacht wird, was darauf hindeutet, dass die Expression von etsrp aber nicht die von tal1, unabhängig von Npas4l auftreten kann. Zur Unterstützung dieser Idee kann etsrp, aber nicht tal1Expression in den meisten fli1a-exprimierenden Zellen in npas $4 \mathrm{l}^{-/-}$-Embryonen beobachtet werden. Dennoch wird der Großteil der etsrp-Expression durch Npas4l reguliert (Marass et al., 2019). 
Diese Daten legen nahe, dass verschiedene Gene unterschiedliche Prozesse während der frühen Endothelentwicklung regulieren. In npas $4^{-/-}$- und etsrp $^{-/-}$ Embryonen differenzieren npas 4 -Reporter exprimierende Zellen nicht zu Endothelzellen und tragen stattdessen zur Skelettmuskelzellpopulation bei. In npas $4 l^{-/}$- und tal1-/--Embryonen können npas4l-Reporter exprimierende Zellen nicht migrieren und tragen stattdessen zu der Bildung der pronephrischen Tubuli bei. Um die Beziehung zwischen diesen Faktoren besser zu verstehen, habe ich getestet, ob die Injektion von etsrp-, tal1-oder lmo2-mRNA verschiedene Aspekte des npas4l-Phänotyps retten würde.

tal1-mRNA-Injektionen reichten aus, um eine Wildtyp-ähnliche vaskuläre

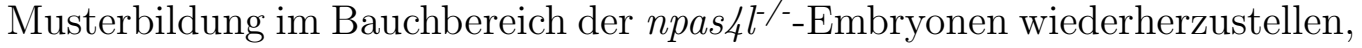
einschließlich der Rettung sowohl der Zellmigration als auch der Differenzierung. Da Npas4l mehrere unterschiedliche transkriptionelle Effektoren hat, war eine so starke Rettung durch nur einen dieser Effektoren unerwartet. In den geretteten Mutanten wurde die bilaterale Population von npas4l-Reporter-positiven pronephrischen Tubuluszellen nicht entdeckt, aber die Anzahl der ektopischen npas4l-Reporter exprimierenden Muskelzellen war im Vergleich zu nicht injizierten npas4l-Mutanten gleichbleibend. Injektionen von etsrp- oder lmo2-mRNA hatten keinen signifikanten Effekt auf die Endothelentwicklung in npas4l-Mutanten. tal1-mRNA-Injektionen in etsrp-Mutanten führten nicht zu einer Rettung der Bauchgefäße, was darauf hindeutet, dass die durch tal1-mRNA vermittelte Rettung des npas4lMutantenphänotyps von der Etsrp-Funktion abhängt. Diese Daten legen auch nahe, dass tal1 die etsrp-Expression fördert. Um diese Hypothese zu testen, habe ich eine quantitative PCR verwendet, um die mRNA-Spiegel von tal1 und etsrp bei 10 Stunden nach Befruchtung und Injektion von npas 4 l-, etsrp-, tal1- und lmo2-mRNA in Wildtypembryonen zu messen. Ich fand heraus, dass tal1 in diesem Stadium nur durch Npas4l induziert werden konnte, während etsrp sowohl durch Npas4l als auch Tal1 induziert werden konnte.

\section{Fazit}

Insgesamt habe ich mehrere Werkzeuge entwickelt, um sowohl das Npas4lProtein nachzuweisen als auch npas4l-exprimierende Zellen in vivo zu verfolgen. Diese Werkzeuge erlaubten mir zu untersuchen, was mit den npas 4 lexprimierenden Zelltypen in npas4l-Mutanten passiert. Ich stellte fest, dass diese Zellen weder differenzieren noch migrieren und stattdessen zu den pronephrischen Tubuli und der Skelettmuskulatur beitragen. Der Migrationsdefekt und der Beitrag zu den pronephrischen Tubuli werden beide durch das Fehlen von tal1 vermittelt, während das Fehlen von etsrp die Tubulogenese 
und Differenzierungsdefekte vermittelt und zu einer Differenzierung von Endothelvorläuferzellen zur Skelettmuskulatur hervorruft. Außerdem habe ich die ersten Wirbeltiermodelle generiert, die komplett frei von Endothelzellen sind und gezeigt, dass sich bei gleichzeitiger Abwesenheit von npas $4 l$ und etsrp oder etsrp und tal1 keine Endothelzellen entwickeln können.

Diese Daten geben einen Einblick in die ersten Schritte der endothelialen Differenzierung. Detaillierte Kenntnisse über die Abstammungsentscheidungen während der Endothelentwicklung können auch bei der Umwandlung adulter Zellen in Endothelzellen im therapeutischen Kontext helfen. Wird es zum Beispiel einfacher sein, pronephrische Tubulus- und Skelettmuskelzellen in Endothelzellen zu konvertieren als andere Zelltypen? Es von Interesse sein, solche Fragen in der Zukunft zu klären. 


\section{English summary}

\section{Introduction}

The formation of blood vessels is essential for vertebrate development and homeostasis, and endothelial cell specification is a key initial step in this process. Most of our knowledge about endothelial cell specification comes from studies in mice and zebrafish. As zebrafish are developing in transparent eggs and are resilient against cardiovascular defects in early development, they represent the ideal model system for the investigation of endothelial cell behavior during early vascular development (Stainier et al., 1996; Isogai et al., 2001).

The earliest known event in endothelial specification in zebrafish is the expression of the bHLH-PAS transcription factor gene cloche/npas4l (Reischauer et al., 2016). cloche/npas 4 l mutants lack most endothelial and hematopoietic cells (Stainier et al., 1995; Liao et al., 1997; Parker and Stainier, 1999). Mechanistically, Npas4l regulates the expression of several targets including the transcription factor genes etsrp, tal1 and lmo2 (Qian et al., 2005; Marass et al., 2019), thereby promoting the formation of endothelial and hematopoietic cells. These endothelial and hematopoietic progenitors in the lateral plate mesoderm move along the ventral side of the somites towards the midline, where they coalesce into a vascular cord that forms the two major axial vessels: the dorsal aorta (DA) and posterior cardinal vein (PCV) (Jin et al., 2005; Helker et al., 2015).

Etsrp is an ETS-transcription factor family member required for endothelial and myeloid specification (Sumanas and Lin, 2006; Pham et al., 2007). In zebrafish etsrp mutants and morphants, endothelial progenitors acquire alternative fates including cardiac and skeletal muscle leading to the absence of most endothelial cells (Palencia-Desai et al., 2011; Chestnut et al., 2020). Global etsrp overexpression in zebrafish embryos leads to an increase in endothelial marker gene expression (Kuan et al., 2009; Veldman et al., 2013). 
TAL1 is a bHLH-transcription factor required for endothelial and hematopoietic development. In mice lacking Tal1, both vascular plexus remodeling and hematopoietic development are impaired (Faloon et al., 2000; Robertson et al., 2000). Similarly, zebrafish tal1 mutants lack the expression of the erythroid marker gata1a and erythrocytes (Liao et al., 1998), and they exhibit vascular defects (Bussmann et al., 2007). Global overexpression of tal1 in npas4l mutants leads to a partial rescue of endothelial and blood cell differentiation (Liao et al., 1998).

LMO2 is a LIM domain containing transcription factor required for hematopoietic development. Both zebrafish and mouse Lmo2 loss-of-function models exhibit defective erythroid development and marker gene expression (Warren et al., 1994; Patterson et al., 2007). Zebrafish lmo2 mutants also display vascular defects that are linked to reduced endothelial cell migration (Weiss et al., 2012; Matrone et al., 2017). LMO2 is part of transcription factor complexes involving TAL1 (Warren et al., 1994; Osada et al., 1995), and it has been reported to function as part of a scaffold for transcription factor complexes (El Omari et al., 2011).

\section{Two knock-in reporter lines for npas 4 l enable the visu- alization of npas $4 l$ expression and detection of Npas4l protein}

I generated a knock-in V5 line to detect the tagged Npas4l on the protein level and a Gal4-VP16 reporter line to visualize and trace npas4l expressing cells in vivo. Both lines can be detected in early stages and are expressed in early endothelial cells. While the Npas4l-V5 signal is weak and transiently detected in the nuclei of early endothelial progenitor cells, the Gal4 reporter line shows a strong and stable expression that allows tracing of npas $4 \mathrm{l}$ reporter positive cells throughout embryonic and early larval development. Both reporter lines are viable and fertile as homozygous carriers of the respective allele and also complement strong npas $4 \mathrm{l}$ mutant alleles suggesting that the knock-ins did not disrupt the endogenous Npas4l function of alleles carrying the reporter.

\section{npas 4 l reporter expressing cells in npas $4 l$ mutants do not migrate to the midline and instead contribute to skeletal muscle and pronephric tubules}

To follow npas $4 l$ reporter expressing cells in npas $4 l$ mutants, I generated a mutant variant of the Gal4 reporter line described in the previous paragraph. 
In this mutant, the region encoding the DNA-binding site of Npas4l carries an in-frame indel that disrupts Npas4l function but retains the reporter expression. This mutant reporter allele fails to complement npas $4 l$ mutants and shows a strong phenotype suggesting it to be a functional null allele.

Phenotypic analyses showed that npas $4 l$ reporter expressing cells in npas $4 l$ mutants do not specify or migrate to the midline. Instead, they contribute to the intermediate mesoderm-derived pronephric tubules and the paraxial mesoderm-derived skeletal muscle. I confirmed these phenotypes by single cell RNAseq on the npas $4 l$ reporter positive cells in $n p a s 4 l^{+/-}$and npas $4 l^{-/-}$ embryos. Together these two alternative cell fates account for the majority of changes observed between the genotypes.

\section{Etsrp, Tal1 and Lmo2 regulate distinct aspects of en- dothelial development}

Npas4l is known to drive the expression of the three transcription factor genes etsrp, tal1 and lmo2 (Marass et al., 2019). I hypothesized that the consequent lack of each of these transcription factors in npas 4 l mutants cause different aspects of the npas 4 l phenotype. Therefore, I generated mutant lines for all three genes and analyzed them in vascular reporter lines as well as in the npas $4 l$ reporter background. These new mutant alleles recapitulate all previously published phenotypes, including the vascular defects and the loss of red blood cells in tal1 (Bussmann et al., 2007) and lmo2 (Weiss et al., 2012) mutants. I observed that endothelial progenitors in etsrp mutants migrate towards the midline but fail to differentiate and undergo vasculogenesis. Some of them appear to contribute to paraxial mesoderm-derived skeletal muscle, as previously reported for other etsrp mutant alleles (Chestnut et al., 2020). In contrast to that, tal1 mutants display a defective migration of npas $4 l$ reporter expressing cells but no increase in npas $4 l$ reporter expressing skeletal muscle cells. Instead, these non-migratory cells contribute to the intermediate mesoderm-derived pronephric tubules. I counted the number of npas4l/Pax2a double positive cells in the pronephric tubules of all four mutants and confirmed an increase in npas $4 l$ and tal1 mutants but not in etsrp or lmo2 mutants. However, many npas 4 l reporter expressing cells in the PCV of lmo2 mutants were also Pax2a+, indicating endothelial differentiation defects. 


\section{fli1 a positive endothelial cells fail to develop in etsrp;tal1 or etsrp;npas 4 l mutants}

npas4l, etsrp and tal1 mutants all exhibit severe endothelial phenotypes. However, some endothelial cells remain in each mutant. The endothelial phenotype is strongest in npas4t/- embryos, but even in these embryos some fli1a positive endothelial cells can be observed in their tail region. It is unclear whether this population of endothelial cells develops independently of Npas4l, Tal1 and Etsrp function or as a consequence of residual tal1 or etsrp expression independent of Npas4l function. To investigate this question, I generated double mutants and looked for the presence of fli1a+ endothelial cells. While fli1a + endothelial cells are clearly present in $n p a s 4^{-/}$and npas $4 t^{-/} ;$tal1 $^{-/-}$embryos, no such cells can be observed in npas $4 l^{-/} ;$etsrp $^{-/-}$ or etsrp $^{-/-} ;$tal1 $^{-/-}$embryos. These data suggest that in zebrafish, endothelial cells cannot develop if npas $4 l$ and etsrp or etsrp and tal1 are both disrupted. While the loss of etsrp leads to stronger defects in npas $4 l$ mutants, there is no additional phenotype caused by the loss of tal1, suggesting that etsrp but not tal1 expression can occur independently of Npas4l. In support of this idea, etsrp but not tal1 expression can be observed in most fli1a expressing cells in npas $_{4} \mathrm{l}^{-}$embryos. Nevertheless, the majority of etsrp expression is regulated by Npas4l (Marass et al., 2019).

These data suggest that distinct genes regulate different processes during early endothelial development. In npas4 $\mathrm{l}^{-/}$and etsrp $^{-/-}$embryos, npas $4 \mathrm{l}$ reporter expressing cells fail to differentiate into endothelial cells and instead contribute to skeletal muscle cells. In npas $4 l^{-/}$and tal1 $^{-/-}$embryos, npas $4 l$ reporter expressing cells fail to migrate and instead contribute to the pronephric tubules. In order to better understand the relationship between these factors, I tested whether injecting etsrp, tal1, or lmo2 mRNA would rescue different aspects of the npas4l phenotype.

\section{tal1 mRNA injections rescue endothelial development in $n p a s 4^{-/-}$embryos in an Etsrp-dependent manner}

tal1 mRNA injections were sufficient to restore wild-type like ISV patterning in npas $4 \mathrm{l}^{-}$- embryos, including the rescue of both cell migration and differentiation. As Npas4l has several distinct transcriptional effectors, such a strong rescue by just one of these effectors was unexpected. In addition, this rescuing ability of tal1 was observed at doses as low as $0.25 \mathrm{pg}$. In these rescued mutants, the bilateral population of npas $4 l$ reporter positive pronephric tubule cells was not detected, but the number of ectopic npas $4 l$ 
reporter expressing muscle cells was not reduced compared with uninjected npas4l mutants. Injections of etsrp or lmo2 mRNA did not have a significant effect on endothelial development in npas $4 \mathrm{l}$ mutants. tal1 mRNA injections into etsrp mutants did not lead to a rescue of ISV patterning, suggesting that the tal1-mediated rescue of the npas $4 l$ mutant phenotype depends on Etsrp function. These data also suggest that tal1 promotes etsrp expression. To test this hypothesis, I used qPCR to measure tal1 and etsrp mRNA levels at $10 \mathrm{hpf}$ after npas4l, etsrp, tal1 and lmo2 mRNA injection into wild-type embryos. I found that tal1 could only be induced by Npas4l at this stage, while etsrp could be induced by both Npas4l and Tal1.

\section{Conclusion}

Taken together, I developed multiple tools to detect several transgenic tools to detect the Npas4l protein as well as to follow npas4l-expressing cells in vivo. These tools allowed me to investigate what happens to the npas $4 \mathrm{l}$ expressing lineage in npas $4 \mathrm{l}$ mutants. I determined, that these cells fail to differentiate or migrate and instead contribute to the pronephric tubules and skeletal muscle. The migration defect and contribution to the pronephric tubules both are mediated through a lack of tal1, whereas tubulogenesis the differentiation defect and skeletal muscle contribution are mediated through the lack of etsrp. Also, I generated the first vertebrate models completely devoid of endothelial cells showing that no endothelial cells can develop in the simultaneous absence of npas4l and etsrp or etsrp and tal1.

These data give insight into the first steps of endothelial differentiation. Detailed knowledge of lineage decisions during endothelial development may also help in converting adult cells into endothelial cells in a therapeutic context. Will it for instance be easier for instance to convert pronephric tubule and skeletal muscle cells into endothelial cells than other cell types? It will be interesting to address such questions in the future. 



\section{Populärwissenschaftliche Zusammenfassung}

Die korrekte Bildung von Blutgefäßen ist ein wichtiger Schritt in der Entwicklung aller Wirbeltiere und erfordert die Entwicklung von mit Blut gefüllten Hohlräumen, die mit spezialisierten Zellen ausgekleidet sind. Diese Zellen entwickeln sich im Zebrafish an der Seite des Embryos und wandern von dort aus in die Körpermitte, wo sie aneinander haften und ein strangartiges Gebilde formen. Innerhalb dieses Strangs formt sich ein Hohlraum und füllt sich mit Blut.

Das Startsignal für die komplexen Prozesse, die dazu notwendig sind, gibt Npas4l: ein Protein das an die DNA bindet und die Gene kontrolliert und koordiniert, die die Bildung von Blugefäßzellen starten. Schaltet man Npas4l aus, dann bilden sich im Embryo weder Blutgefäße noch Blut. Injiziert man mehr Npas4l in einen Embryo, dann bildet dieser auch mehr Blutgefäßzellen.

Ich habe genetische Werkzeuge geschaffen, mit denen ich die Zellen, in denen Npas4l vorhanden ist in lebenden Fischen zum Leuchten bringen kann. Dadurch konnte ich experimentell zeigen, was mit Zellen passiert, die bei einem Funktionsverlust von Npas4l nicht zu Blutgefäßzellen werden können. Ich habe herausgefunden, dass diese Blutgefäßvorläuferzellen stattdessen zu der Skelettmuskulatur und Nierenzellen werden. Außerdem zeigen meine Experimente, dass diese Effekte durch verschiedene von Npas4l kontrollierte Gene ausgelöst werden.

Diese Erkenntnisse liefern Hinweise darauf, welche Gewebe und Zelltypen den Blutgefäßzellen am ähnlichsten sind und eventuell wieder umprogrammiert werden könnten, um neue Blutgefäße zu bilden. Diese Frage ist zum Beispiel nach größeren Verletzungen oder Operationen relevant, da dort unter anderem die Fähigkeit des Körpers neue Blutgefäße zu bilden die Heilung des Gewebes limitiert. 



\section{Popular science summary}

The correct formation of blood vessels is an important step in the development of all vertebrates and requires the development of blood-filled cavities lined with specialized cells. In zebrafish, these cells develop on the side of the embryo from where they migrate to the center of the body. There they adhere to each other and form a strand-like structure. Within this strand, cavities form and fill with blood.

The starting signal for these complex processes is given by Npas4l, a protein that binds to DNA and coordinates the genes that start the formation of cells lining the blood vessels. If Npas4l is switched off, neither blood vessels nor blood form in the embryo. Respectively, if you inject more of it into an embryo, it will form more blood vessel cells.

I created genetic tools that turn the cells in which Npas4l is present fluorescent in living fish. This allowed me to investigate what happens to the cells that fail to become blood vessel cells when Npas4l loses function. I found that these blood vessel progenitor cells become skeletal muscle and kidney cells instead. Moreover, my experiments show that these effects are caused by the action of different Npas4l-controlled genes.

These findings provide clues as to which tissues and cell types most resemble blood vessel cells and might be reprogrammed to form new blood vessels. This question is relevant, for example, after major injury or surgery, where tissue healing is limited in part by the ability of the body to form new blood vessels. 



\section{Chapter 1}

\section{Introduction}

The cardiovascular system is the first organ system that becomes functional in the vertebrate embryo. This thesis focuses on the earliest step in vascular development - the specification of endothelial cells and some of the most important transcription factors involved in this process. The introduction gives a brief overview over the evolutionary origin of the vascular system and endothelial cells (section 1.1) and vascular development in zebrafish (section 1.2), before focusing on the most important genes involved in endothelial specification (section 1.3).

\subsection{Evolution of the vascular system}

The increasing size and complexity of animals goes hand in hand with the development of more complex systems that facilitate the directed distribution of molecules beyond free diffusion. A phylogenetic schematic of vascular development is provided in Figure 1.1. In invertebrate animals, various designs of both open and closed vascular systems without an endothelial lining are found. The first appearance of a primitive closed circulatory system is evident in annelids (Johnston and Johnson, 1902). In annelids, peristaltic contractions of the vascular structures move blood devoid of red blood cells or haemoglobin through the body. Open systems are found in arthropods and non-cephalopod mollusks. They consist of hemolymph that is pumped through a contractile heart into a mesoderm-derived body cavity called the hemocoel, where it mixes with the interstitial fluid and flows freely around the organs. This system has disadvantages however, as it perfuses one organ after the other so that organs proximal to the heart receive most nutrients whereas distal organs receive most waste products. Also, the velocity and pressure of the fluid coming from the heart drops when entering the hemocoel. 
Cephalopods, chordates and vertebrates all possess closed circulatory systems (Monahan-Earley et al., 2013, Figure 1.1).

In all invertebrates and cephalochordates, the vascular system is not lined by endothelial cells. Instead, their vascular lumen is lined by extracellular matrix and contains amoebocytes but no red blood cells or haemoglobin

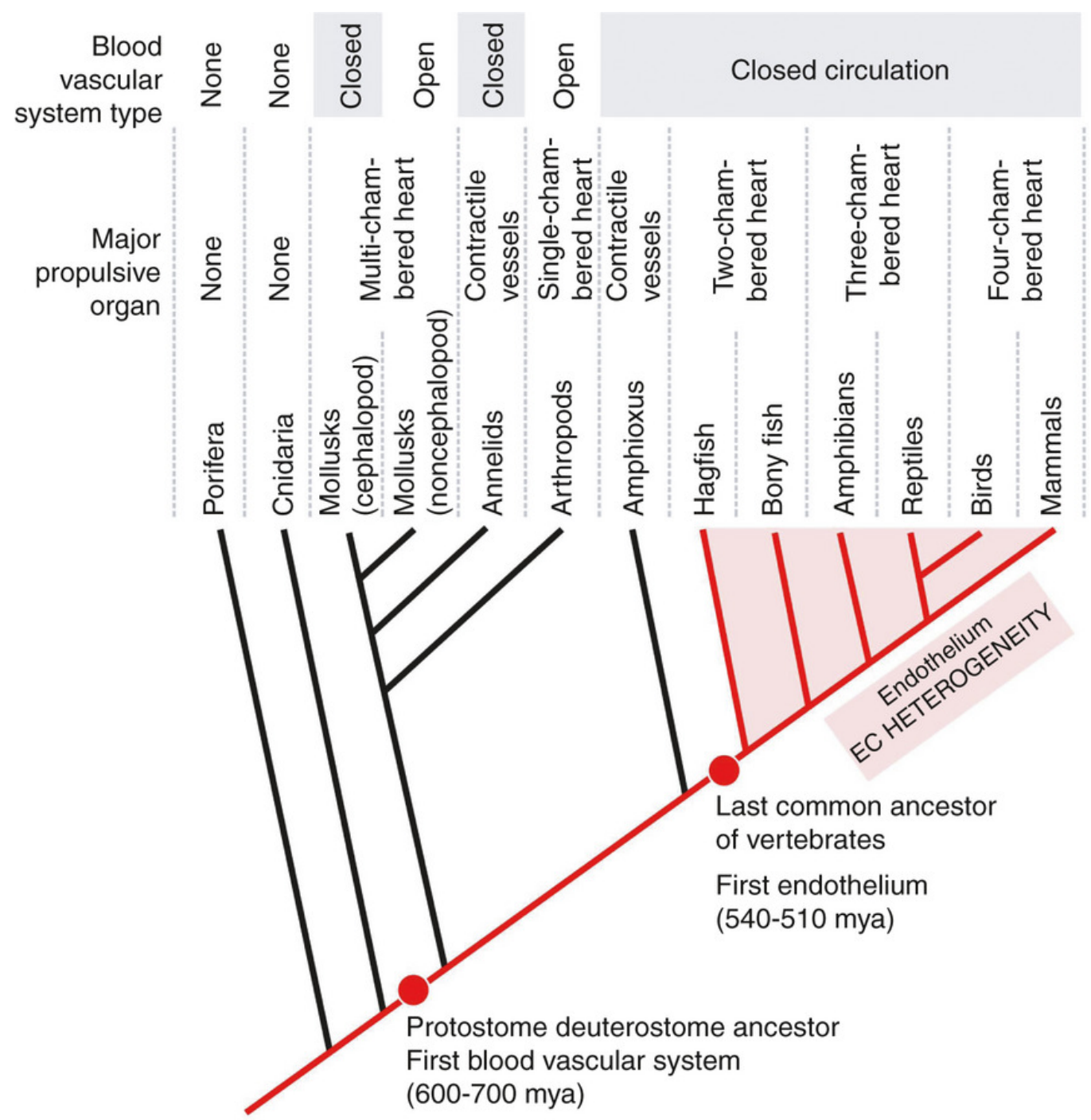

Figure 1.1: Phylogenetic schematic of blood and vascular system development. Blood vascular system type and major propulsive organ. Evolutionary time is not drawn to scale. EC: endothelial cells, mya: million years ago. Reprinted from Journal of Thrombosis and Haemostasis, 11 (Monahan-Earley et al., 2013), Figure 7 with permission from John Wiley and Sons. 
(Monahan-Earley et al., 2013). Endothelial cells with an apico-basal polarity that are firmly attached to the luminal side of the basal membrane and connect through cell adhesion molecules are first found in early vertebrates such as the hagfish (Yano et al., 2007).

In vertebrates themselves, many functional components such as adhesion molecules like Cdh5 are highly conserved and shared between all vertebrates. Similarities between vertebrate model species are often highlighted as they ease the transfer of basic discoveries to other species. However, especially for early endothelial transcription factors and guidance cues, remarkable differences in functionality can be observed that are equally important to understand endothelial development in different model organisms.

The first example of such differences is Npas4l itself, which is found in most vertebrates but not in mammals (Ingo Ebersberger, collaboration and personal communication). Given the strong phenotype npas $4 l$ mutations cause in zebrafish, it is very surprising that mammals lost this gene. Various other genes show different defects in mouse mutants compared to zebrafish mutants. In some cases this can be attributed to the partial genome duplication present in teleost fish and the consequent redundancy of many genes, but in other cases the reasons for the observed differences are unclear.

One example for such an unclear difference is the ETS transcription factor ETV2. It is currently debated whether this mammalian transcription factor has an orthologue in zebrafish (Ingo Ebersberger, collaboration and personal communication), but even if zebrafish Etsrp and mammalian ETV2 are orthologues, the phenotypes caused by mutations of these genes are remarkably different: While mouse Etv2 mutants lack blood and endothelial cells, zebrafish etsrp mutants display only endothelial and myeloid specification defects but an increased number of red blood cells (Chestnut et al., 2020). One reason for that appears to be that mouse ETV2 is a direct upstream regulator of Tal1 (Liu et al., 2015), whereas in zebrafish, tal1 expression is mainly induced by Npas4l in early stages (Marass et al., 2019). Therefore, the phenotype of mouse Etv2 mutants is more similar to the one observed in zebrafish npas 4 l mutants than it is to the etsrp mutant phenotype.

Also other mutants display different phenotypes during vascular development in mouse and zebrafish. A recent example is the lack of a phenotype in zebrafish tie2 mutants, that show no obvious vascular defects (Jiang et al., 2020), whereas the mouse Tie2 mutants display strong global endothelial 
defects (Dumont et al., 1993, 1994). Similarly, also the VEGF-VEGFR2 signaling axis, which guides angioblast migration in mice (Shalaby et al., 1995; Ferrara et al., 1996, 2003), is not required during angioblast migration in zebrafish (Habeck et al., 2002; Helker et al., 2015; Hogan and Schulte-Merker, 2017). Similarly, mouse Fli1 mutants display strong vascular and hematopoietic defects (Spyropoulos et al., 2000), whereas zebrafish fli1a/fli1b double mutants do not display morphological defects (Craig et al., 2015).

All in all it is important to transfer knowledge to other model organisms, but it appears to be particularly important in zebrafish to keep speciesspecific effects of the various factors in mind that are involved in vascular development. The species-specific variations in mutant phenotypes of early expressed endothelial genes suggest that the developmental mechanisms regulating endothelial specification and vasculogenesis may be plastic.

\subsection{A cross-section through vascular develop- ment in zebrafish}

This section gives a brief overview of the early developmental events that lead to the formation of the zebrafish vasculature. It also summarizes key events that happen at later stages. As this is a vast field with many specialized areas, this section does not intend to be a complete review of all the complex processes involved, but to put the induction of an endothelial cell fate in a broader context.

\subsubsection{Endothelial cell origin}

The main source of endothelial cells in zebrafish is a bilateral cell population in the anterior and posterior lateral plate mesoderm. (Reischauer et al., 2016; Prummel et al., 2019). The mesoderm develops from the mesendoderm with the involvement of factors such as eomesodermin (Xu et al., 2014), brachyury (Halpern et al., 1993; Schulte-Merker et al., 1994) and BMPsignaling (Mullins et al., 1996; Kishimoto et al., 1997). The lateral plate mesoderm itself gives rise to progenitors of myeloid, cardiopharyngeal, intestinal smooth muscle, pectoral fin, endothelial and blood progenitors (Prummel et al. 2019, Figure 1.2). Whether pax2a positive pronephric mesoderm should be considered to be a lateral plate mesoderm-derived (Prummel et al., 2019) or an intermediate mesoderm-derived (Perens et al., 2016) cell population is debated. 


\section{m}

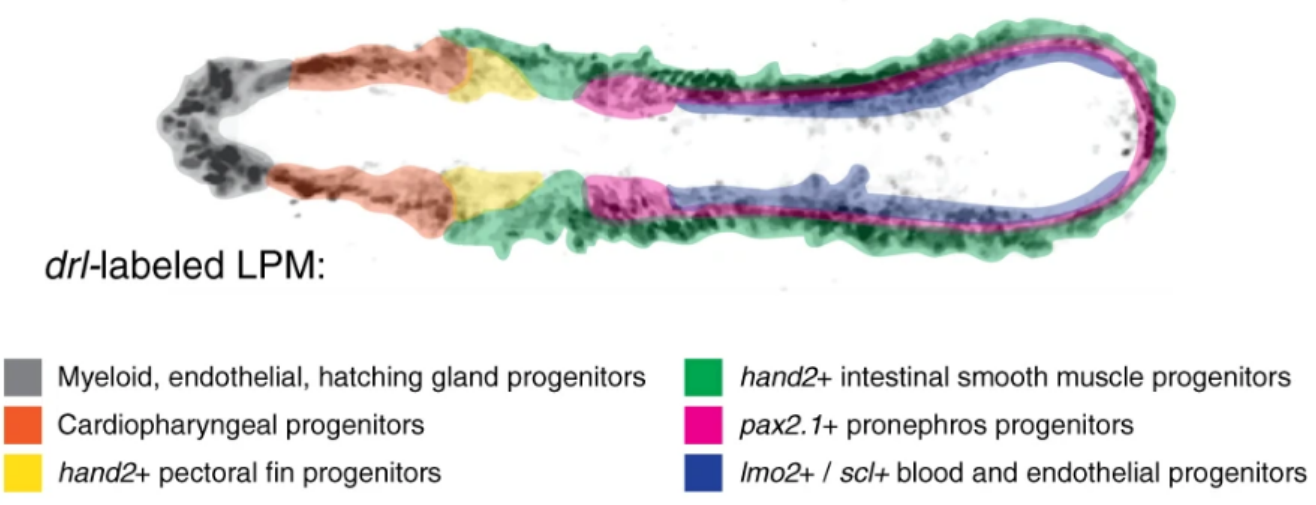

Figure 1.2: Schematic representation of different territories of the lateral plate mesoderm and cell fates they give rise to. Dorsal view of a flat mount projected embryo at early somite stages. Anterior side on the left. Adapted from Prummel et al. (2019), Figure 1m. License: CC BY 2.0.

Although the lateral plate mesoderm is the main origin of endothelial cells in zebrafish, other mesodermal sources of specialized endothelial cells exist. In some species they are the main source of certain types of specialized endothelial cells. The paraxial mesoderm has been suggested to be an additional source of endothelial cells in various species (Couly et al., 1995; Wilting et al., 2000; Wasteson et al., 2008; Nguyen et al., 2014; Stone and Stainier, 2019). In zebrafish, the interaction with the paraxial mesoderm is required for the differentiation of hematopoietic cell progenitors (Rohde et al., 2004), and the paraxial mesoderm itself contains bipotent progenitors that can give rise to hematopoietic stem and progenitor cells or muscle progenitors (Nguyen et al., 2014; Sahai-Hernandez et al., 2020). Also the endoderm has been suggested but not yet shown to give rise to endothelial cells.

\subsubsection{Specification of endothelial cells}

Vascular development starts with the specification of endothelial cells and their assembly into the major axial vessels (Jin and Patterson, 2009; Ellertsdóttir et al., 2010; Bautch, 2011; Herbert and Stainier, 2011; Bautch and Caron, 2015; Hogan and Schulte-Merker, 2017). In zebrafish the initial step of endothelial specification is mediated by the transcription factor Npas4l, that induces the development of the first endothelial cells in the lateral plate mesoderm (Reischauer et al., 2016, Figure 1.3A). Upon npas 4 l expression 


\section{A Angioblast specification}

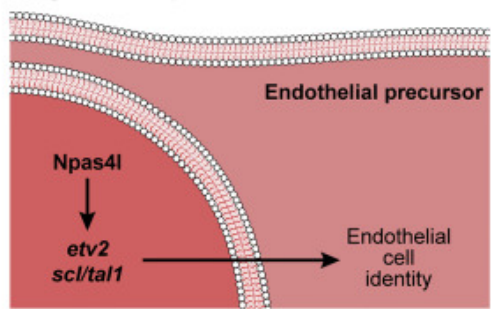

C Transverse

$10 \mathrm{~s}$

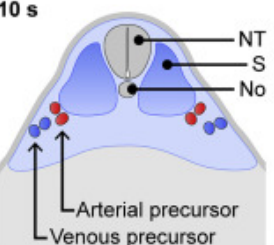

$11 \mathrm{~s}$

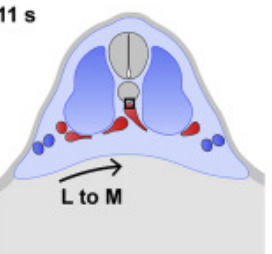

$16 \mathrm{~s}$

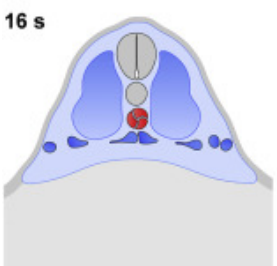

$22 \mathrm{~s}$

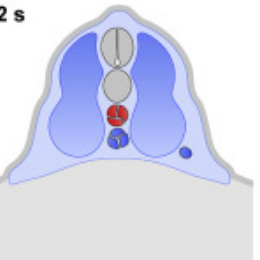

Lateral
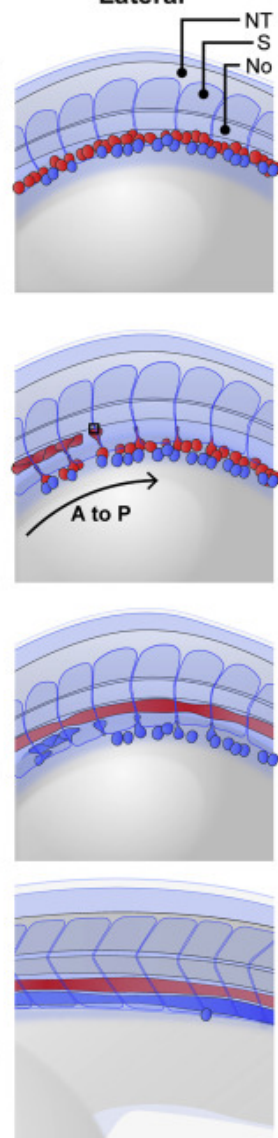

B 10 somites Lateral

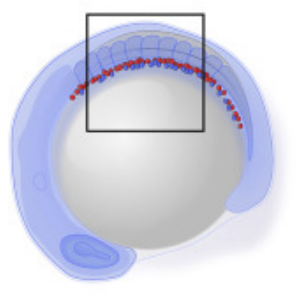

Dorsal
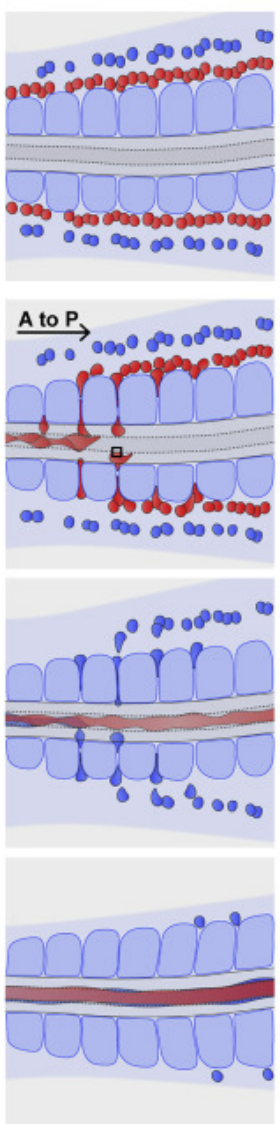

D Angioblast migration

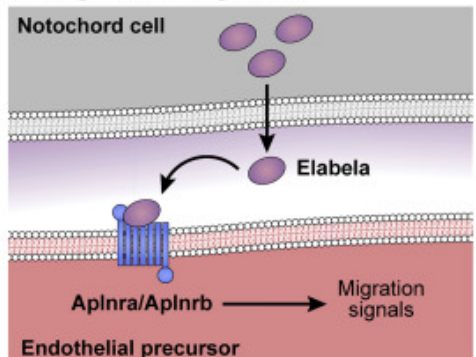

E 22 somites Latera

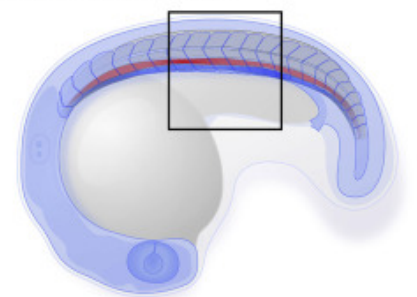

F Tubulogenesis

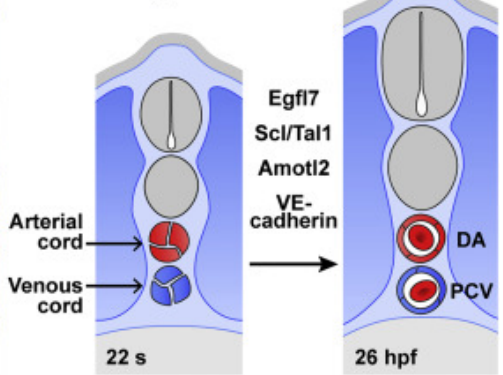

Figure 1.3: Schematic representation of vasculogenesis in zebrafish. (A) Endothelial specification is induced by transcriptional activity of Npas4l. (B) Until $14 \mathrm{hpf}$ (10 somite stage), endothelial progenitors are located on the lateral sides of the somites. (C) From 14 to 20 hpf (10-22 somite stage), angioblasts migrate ventral to the somites towards the midline. Anterior and arterial endothelial progenitors move earlier than venous and posterior progenitors. (D) The migration of endothelial cells is guided by Elabela secretion from the notochord and sensed by Aplnra/Aplnrb receptors on the endothelial progenitor cells. Figure legend continued on the next page. 
Figure 1.3: (E) At 20 hpf (22 somite stage) endothelial progenitors have coalesced into vascular cords. (F) The cells then undergo tubulogenesis and fill with blood. Reprinted from Developmental Cell, Vol 42, Issue 6, (Hogan and Schulte-Merker, 2017), Figure 1 with permission from Elsevier.

during late gastrulation and early somite stages, it triggers complex morphogenetic processes leading to the assembly of a lumenized vascular structure lined with highly specialized endothelial cells (Jin et al., 2005; Helker et al., 2015).

\subsubsection{Endothelial migration and tubulogenesis}

Around 14 hours post fertilization (hpf, also known as 10 somite stage), the endothelial and hematopoietic progenitors in the lateral plate mesoderm start to move along the ventral side of the somites towards the midline (Figure 1.3B-C). They coalesce into a vascular cord (Figure 1.3E) that lumenizes and fills with blood (Figure 1.3F), thereby forming the two major axial vessels, the dorsal aorta (DA) and posterior cardinal vein (PCV) (Eriksson and Löfberg, 2000; Jin et al., 2005; Helker et al., 2015). The migration is guided by notochord derived Elabela sensed through Aplnra/Aplnrb of endothelial progenitor cells (Helker et al., 2015; Figure 1.3D). The developmental processes described until this point are summarized under the term "vasculogenesis" - the de novo formation of blood vessels. Their correct and timely completion are the necessary requirement for the formation of a closed vascular loop and the numerous processes that refine the vascular system such as angiogenic sprouting, vascular heterogeneity and, in a wider sense, all embryonic processes that require a reliable supply with oxygen, nutrients and circulating factors. Figure $\mathbf{1 . 4}$ depicts the vasculature at approximately 1.5 dpf, shortly after blood starts circulating in the developing vascular system.

Vasculogenesis describes the process of de novo formation of tubular structures whereas angiogenesis requires pre-existing vessels from which new vascular structures sprout. It is surprising that compared to angiogenesis the sprouting of blood vessels from pre-existing ones, the process of vasculogenesis is not very well studied. However, researching vasculogenesis in mammalian model systems like the mouse model faces a number of unique technical challenges like the visualization of dynamic processes in very early (E7.5-E8.5) mouse embryos. Also, strong defects in such early stages and the consequent lack of vascular structures in the placenta lead to the abortion of the embryos before most defects would become obvious. In contrast, 
zebrafish embryos develop in transparent, extraembryonic eggs and are very resilient against vascular defects during the period most informative to study endothelial specification and vasculogenesis, allowing us to study these processes in real time with high resolution (Stainier et al., 1996; Isogai et al., 2001). Combined with the latest advances in zebrafish genome editing these advantages now enable the tracing of endothelial progenitors in living animals.

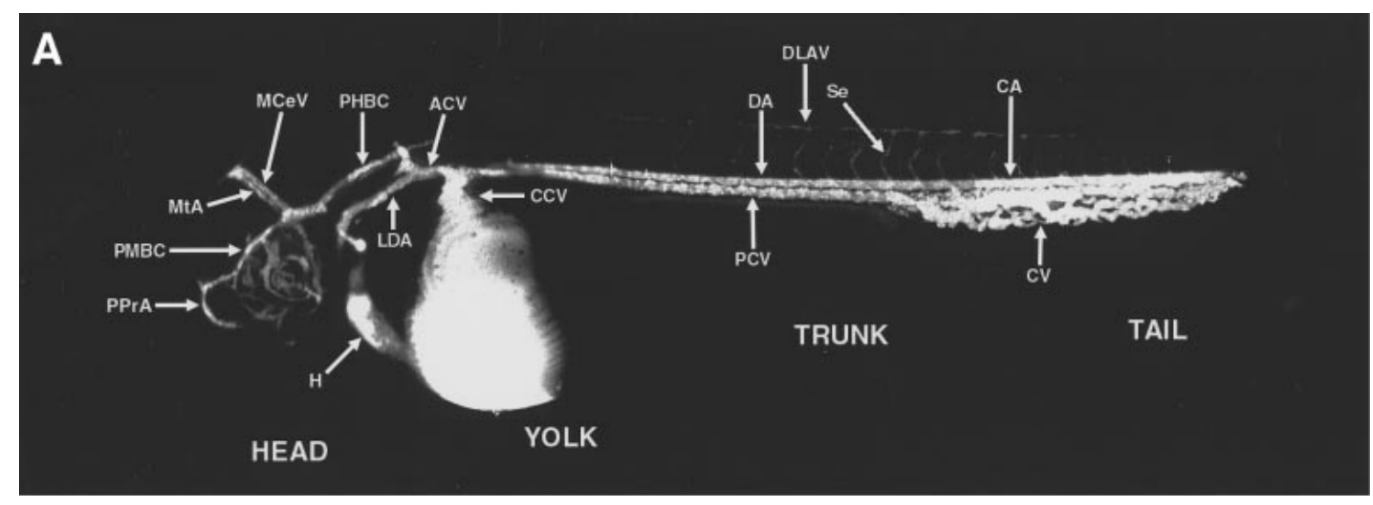

Figure 1.4: Angiogram of a laterally mounted zebrafish embryo at approximately 1.5 dpf. Anatomical structures from anterior to posterior: PPrA: primitive prosencephalic artery, PMBC: primordial midbrain channel, MtA: metencephalic artery, $\mathrm{MCeV}$ : middle cerebral vein, $\mathrm{H}$ : heart, PHBC: primordial hindbrain channel, LDA: lateral dorsal aorta, ACV: anterior (rostral) cardinal vein, CCV: common cardinal vein, PCV: posterior (caudal) cardinal vein, DA: dorsal aorta, DLAV: dorsal longitudinal anastomotic vessel, Se: intersegmental vessel (ISV), CA: caudal artery, CV: caudal vein. Reprinted from Developmental Biology, Vol 230, Issue 2, (Isogai et al., 2001), Figure 2A with permission from Elsevier.

\subsubsection{Angiogenesis and ISV formation}

Before the heart starts pumping, angiogenic sprouting of intersomitic arteries from the DA towards the dorsal side of the trunk begins (Isogai et al., 2001) guided by interactions between Vegfr2 and Vegfaa, whereas venous sprouting events are mediated by Bmp-signaling (Wiley et al., 2011). Sprouts start to be seen on the dorsal side of the DA, where endothelial tip cells form filopodia and are pushed along by stalk cells in between the somites to form the dorsal longitudinal anastomotic vessel dorsal to the neural tube. In the selection of tip and stalk cells and the determination of arteriovenous identity, Notch signaling plays a critical role (Siekmann and Lawson, 2007; Phng and Gerhardt, 2009; Quillien et al., 2014). 


\subsubsection{Vascular maturation}

The first ISV sprouts originate from the DA and are not connected to any vein. In a second wave of sprouting at around $30 \mathrm{hpf}$, about $50 \%$ of the ISV disconnect stochastically from the DA and re-connect to the PCV leading to a network of vessels that perfuse the trunk and tail region of the zebrafish embryo (Geudens et al., 2019). Parallel to these processes, the CCV develops through lumen ensheathment (Helker et al., 2013), and the major brain vessels develop through angioblast cluster-derived angiogenesis (Proulx et al., 2010). Whereas first lymphatic vessels sprout from specialized endothelial cells in the veins (Nicenboim et al., 2015) in a Vegfc/Vegfr3 signaling dependent manner (Küchler et al., 2006), facial lymphatics also recruit additional angioblasts to the tip of the sprouting lymphatic vessels (Okuda et al., 2012). In addition to endothelial development, perivascular mural cells start to cover the vasculature and add another layer of complexity to the vascular system (Santoro et al., 2009; Wang et al., 2014; Ando et al., 2016). The end result is a highly heterogeneous and organ-specific vasculature.

\subsection{Transcription factors inducing endothe- lial specification}

At the tailbud stage of zebrafish development, the expression of the earliest hematovascular transcription factor npas $4 \mathrm{l}$ can be observed in rows of cells located in the anterior and posterior lateral plate mesoderm, inducing endothelial differentiation (Reischauer et al., 2016). This is the earliest event clearly associated with vascular development in this model organism (expression shown in Figure 1.5).

Mechanistically, Npas4l acts by directly promoting the expression of the transcription factor genes etsrp, tal1 and lmo2 (Marass et al., 2019). The three transcription factors encoded by these genes in turn strongly promote the development of endothelial and hematopoietic cells.

\subsubsection{Npas4l}

To date, the earliest known event in endothelial specification in zebrafish is the expression of the bHLH-PAS transcription factor gene cloche/npas $4 \mathrm{l}$ (Reischauer et al., 2016). However, for a long time, mutants of npas $4 \mathrm{l}$ were known as cloche mutants, describing a distinct bell-shape of the heart (Figure 1.6) caused by the absence of endocardium (Stainier et al., 1995). 


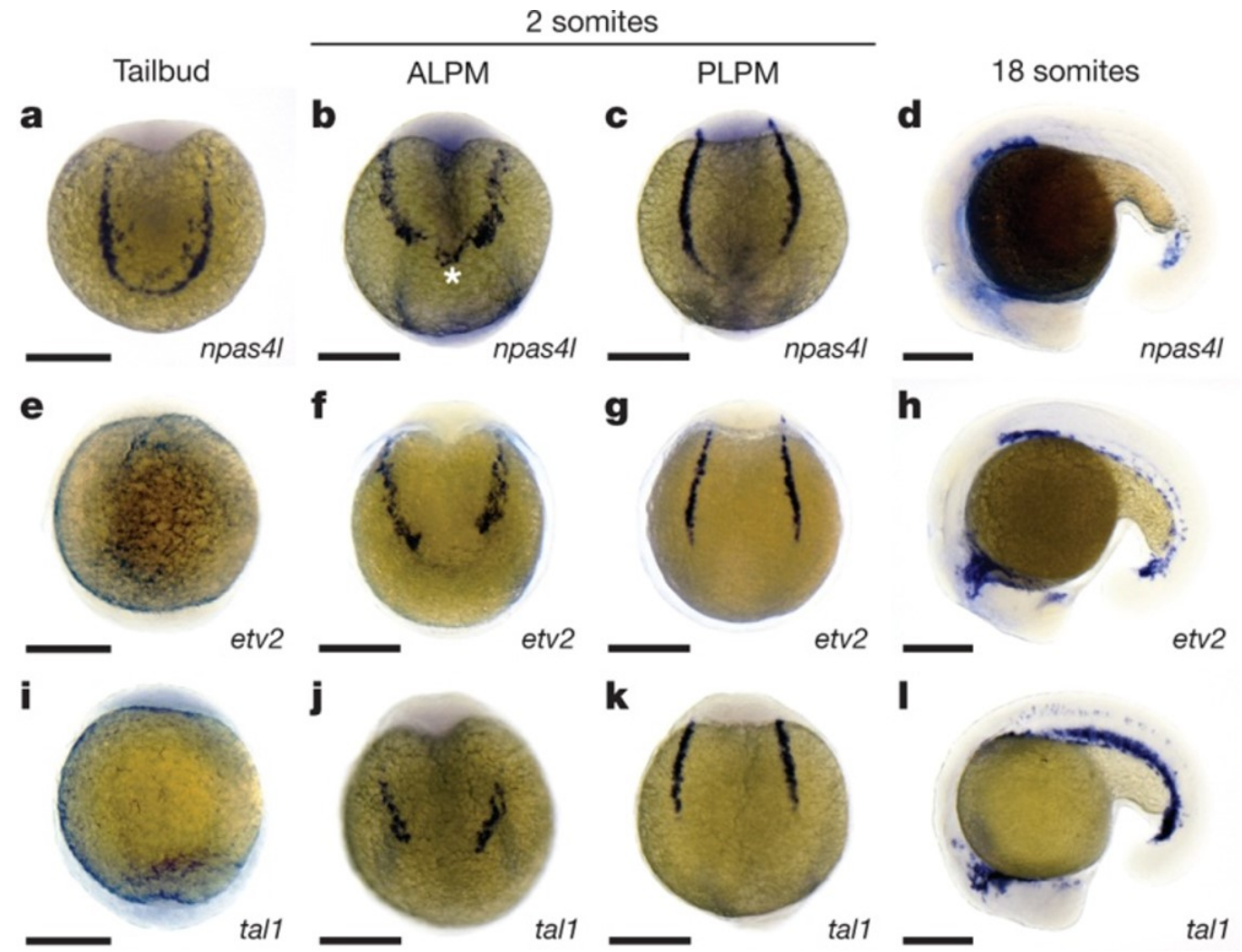

Figure 1.5: Expression of npas4l precedes the expression of etsrp(etv2) and tal1. npas 4 l expression (a-d) can be observed in wholemount in situ hybridization from the tailbud stage (10 hpf) onwards whereas etsrp (e-h) and tal1 (i-l) expression is first observed at the 2 somite stage $(10.33 \mathrm{hpf})$. Scale bars: $200 \mu \mathrm{m}$. Reprinted with permission from Springer Nature: Nature (Reischauer et al., 2016), Figure 3.

cloche/npas $4 \mathrm{l}$ mutants lack most endothelial and hematopoietic cells (Stainier et al., 1995; Liao et al., 1997; Thompson et al., 1998; Parker and Stainier, 1999).

The first cloche $^{m 39}$ mutant allele that has been investigated is a spontaneous chromosomal aberration discovered in a semi-wild population of Indonesian zebrafish (Stainier et al., 1995). In two independent ENU screens, additional cloche alleles were discovered: The strong cloch $e^{s 5}$ allele was identified in the San Francisco screen shortly after the discovery of the $m 39$ allele, and the weak cloche ${ }^{172}$ allele was later identified in a screen for lyC-deficient fish (Ma et al., 2011). 

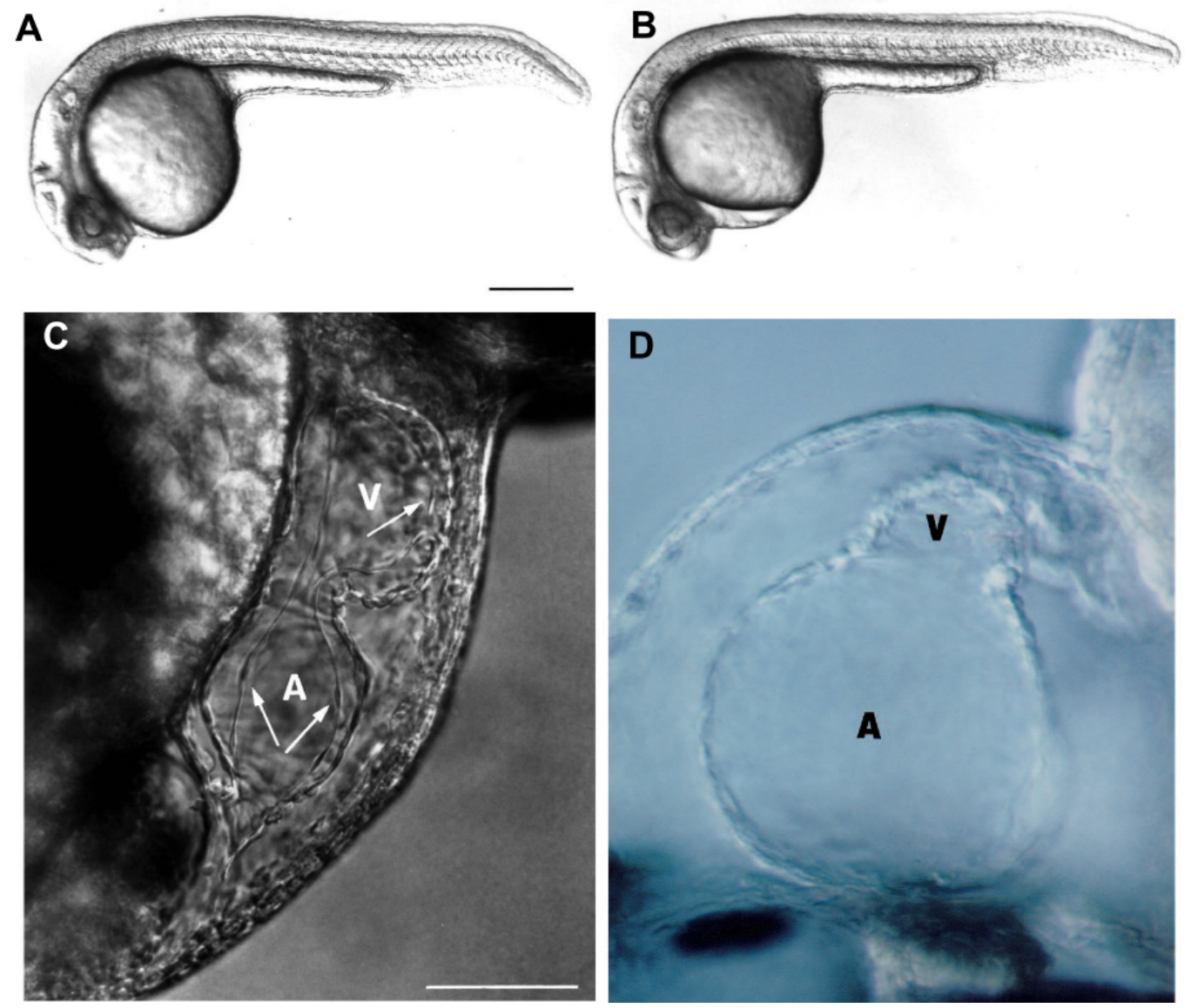

Figure 1.6: cloche mutant phenotype. cloche mutants specifically lack endothelial cells. Wildtype (A) and homozygous cloche $e^{m 39}$ mutants are morphologically similar at 24 hpf. At 40 hpf, a distinct endocardial layer (arrows) can be clearly observed in the wild-type (C) but not in the mutant (D) heart, suggesting the absence of endothelial cells. Morphologically, mutant hearts are characterized by an enlarged atrium and a collapsed ventricle. A: atrium, V: ventricle. Scale bars: $200 \mu \mathrm{m}$ (A-B), $50 \mu \mathrm{m}$ (C-D). Reproduced and adapted with permission by Development (Stainier et al., 1995).

Mechanistically, cloche was determined to be cell-autonomously required for the formation of endothelial cells (Stainier et al., 1995) upstream of $f l k 1$ (encoding Vegfr2) (Liao et al., 1997). A cell-non autonomous requirement for the development of blood was detected as well (Parker and Stainier, 1999). While many studies investigated the loss of endothelial cells in this mutant and the genes associated with it (Qian et al., 2005; Sumanas et al., 2005), only little is known about what happens to the cells that do not become endothelial cells or blood in the cloche mutant. One effect that has been reported before is that npas 4 l mutants have more myocardium which sug- 
gests that endothelial progenitors in the cloche mutant may contribute to this cell type (Schoenebeck et al., 2007). Remaining endothelial cells in cloche mutants express genes associated with neurogenesis and somitogenesis, suggesting defects in their specification or a cellular origin distinct from conventional endothelial cells (Pak et al., 2020). Besides functional research on cloche mutants themselves, they were often used as an avascular and bloodless model system, to investigate the lack of endothelial and blood cells on developmental (Rasmussen et al., 2015; Fortuna et al., 2015; Posner et al., 2019) and regenerative (Hasegawa et al., 2015) processes.

When publishing the initial mapping of cloche to the telomeric region of chromosome 13 Liao et al. (2000) predicted that it would be hard to identify this gene by positional cloning due to its position in a repetitive region. Nevertheless, the interest in the identity of cloche and the need for tools to investigate it was great enough to fuel a continued search for its identity which resulted in ever more precise mappings. With the ability to profile the transcriptome by next generation sequencing, it became possible to compare the transcriptomes of wild-type and cloche $e^{m 39}$ mutant embryos, in which the region containing this gene was completely missing and, therefore, no reads matching genes located on the missing region would be detected. Mutants for all the genes discovered by this method were cloned in the Stainier lab, but the gene causing the cloche phenotype was still not found. Only with the release of the zebrafish genome assembly GRCz10 (Genome Reference Consortium, PRJNA11776), a new unmapped gene lost in the m39 mutant was discovered that also mapped to the cloche locus. On September $25^{\text {th }}$ 2015, lab gossip spread quickly, that Didier had been visiting the zebrafish screening room himself, indicating that this meant cloche might finally have been found. Indeed, Reischauer, Stone, Villasenor, Chi, Jin, Martin, Lee, Fukuda, Marass, Witty, Fiddes, Kuo, Chung, Salek, Lerrigo, Alsio, Luo, Tworus, Augustine, Mucenieks, Nystedt, Giraldez, Schroth, Andersson, and Stainier (2016) published shortly after, that npas4l, a gene encoding a bHLH transcription factor is responsible for the cloche phenotype. This success showed the potential of approaching long-standing problems with creative ideas and new technologies, in this case next generation sequencing and and the ability to generate mutants quickly and cheaply through CRISPR/Cas9 genome editing.

Knowing the identity of cloche/npas 4 l, predictions regarding potential binding motifs and interaction partners could be made for the first time. As a type 1 bHLH-PAS (basic helix-loop-helix-PER-ARNT-SIM) transcription 
factor, Npas4l is part of the larger HLH transcription factor family (Massari and Murre, 2000). It shares the common domain architecture of bHLHPAS transcription factors consisting of a DNA-binding bHLH domain, two PAS-domains and regulatory domains such as transactivation domains in the C-terminus (Bersten et al., 2013; Fribourgh and Partch, 2017). bHLH transcription factors bind to the DNA by forming heterodimers with type 2 aryl hydrocarbon receptor nuclear translocator (AHR/ARNT) proteins. In zebrafish, five of these proteins are present, but which one of them interacts with Npas4l is not known.

In addition to predictions, the discovery of npas4l enabled several functional experiments, such as the search for potential targets using CHIP sequencing and mRNA overexpression. Such analyses lead to the rapid identification of the direct transcriptional targets of Npas4l - etsrp, tal1 and lmo2 (Reischauer et al., 2016; Marass et al., 2019) and thereby to a basic mechanism of Npas4l function (Figure 1.3A). Moreover, it led to the discovery of npas $4 \mathrm{l}$ orthologues in other species such as the chicken (Wei Weng et al., 2020) and the identification of enhancers upstream of npas 4 l, that regulate npas $4 l$ expression in different cell types such as endothelial cells and skeletal muscle (McGarvey et al., 2020).

\subsubsection{Etsrp}

Etsrp is an ETS-transcription factor family member required for endothelial and myeloid specification (Sumanas and Lin, 2006; Pham et al., 2007). The gene was first identified through microarrays of cloche mutants (Sumanas et al., 2005). In zebrafish etsrp mutants and morphants, endothelial progenitors including endocardial cells acquire alternative fates including cardiac and skeletal muscle, leading to the consequent absence of most endothelial cells (Palencia-Desai et al., 2011; Chestnut et al., 2020; Capon and Smith, 2019). In addition to vascular defects also the number of myeloid cells such as macrophages and granulocytes is reduced in the mutants (Sumanas et al., 2008), whereas the number of red blood cells is increased (Chestnut et al., 2020). Although such an effect could be explained by a cell non-autonomous mechanism, it has been suggested based on morpholino studies that hemangioblasts may need to down-regulate etsrp in order to commit to the hematopoietic cell fate (Takeuchi et al., 2015). Global etsrp overexpression in zebrafish embryos on the other hand leads to an increase in endothelial marker gene expression (Kuan et al., 2009). Such overexpression can also lead to the induction of endothelial gene expression in skeletal muscles (Veldman et al., 2013). 
During endothelial development, etsrp is mainly induced by direct transcriptional activity of Npas4l. However, it has also been shown convincingly to be a direct downstream target of foxc1a and foxc1b as well (Veldman and Lin, 2012). Interactions and functional overlap between etsrp and fliib (Baltrunaite et al., 2017), clec14a (Pociute et al., 2019) and ets1 (Casie Chetty and Sumanas, 2020) have been reported.

As mammalian ER71/ETV2 caused the induction of vascular markers as well when overexpressed in zebrafish and alignments of these genes showed some similarity, zebrafish etsrp and mammalian ETV2 were long seen as orthologues (Sumanas et al., 2008). Indeed, the phenotypes of these mutants are similar in many ways such as the decreased number of endothelial cells but increased number of cardiomyocytes (Palencia-Desai et al., 2011; Liu et al., 2012). However, important functional differences between these genes need to be considered as well.

In mice, unlike in zebrafish, Tal1 is a direct target of ETV2 (Liu et al., 2015). Also, unlike zebrafish etsrp mutants, mouse Etv2 mutants, do not develop Gata1a + blood cells (Lee et al., 2008; Koyano-Nakagawa and Garry, 2017). In this sense, mouse Etv2 phenotype is more similar to the npas4l than to the etsrp phenotype in zebrafish. In avian species such as the chicken, Etv2 is completely missing and Tal1 and Lmo2 are regulated through Npas4l as they are in zebrafish (Wei Weng et al., 2020). While npas 4 l does not have an orthologue in mammals, Etv2 may have acquired its functionality in the mammalian lineage. For these reasons, functional equivalence between zebrafish Etsrp and mouse ETV2 should not be assumed without further investigation.

\subsubsection{Tal1}

TAL1 is a bHLH-transcription factor required for endothelial and hematopoietic development. In mice lacking Tal1, both vascular plexus remodeling and hematopoietic development are impaired (Shivdasanl et al., 1995; Robb et al., 1995; Faloon et al., 2000; Robertson et al., 2000). Similarly, zebrafish tal1 mutants lack the expression of the erythroid marker gata1a and consequently erythrocytes (Liao et al., 1998), and they exhibit vascular defects (Bussmann et al., 2007). Global overexpression of tal1 in npas4l mutants leads to a partial rescue of endothelial and blood cell differentiation (Liao et al., 1998). 
During endothelial specification, tal1 expression is directly induced by Npas4l (Marass et al., 2019) but can also be induced by overexpression of hhex (Liao et al., 2000). In early stages, it is expressed in both endothelial and hematopoietic cells (Zhang and Rodaway, 2007). The overexpression of tal1 partially rescues the cloche phenotype (Liao et al., 1998) and leads to the conversion of mesodermal lineages into ectopic blood at the expense of other mesodermal structures such as the intermediate, paraxial and cardiac mesoderm (Gering et al., 1998, 2003). In the absence of gata1a, these ectopic blood cells do not form and ectopic endothelial cells can be observed instead. Similarly, in mouse cell culture experiments TAL1 was found to repress mesodermal lineages other than blood (Chagraoui et al., 2018).

Besides its roles in early hematovascular specification, Tal1 also influences endocardial identity and migration. Zebrafish tal1 mutants specify endocardial cells but these cells accumulate in the ventricle as they fail to migrate and to cover the rest of the heart (Bussmann et al., 2007). Molecularly, intercellular junctions and the maintenance of endocardial identity have been found to be impaired in tal1 mutants (Schumacher et al., 2013).

Although tal1 is usually seen in context with lmo2 as part of the same transcription factor complex (Patterson et al., 2007), it rescues early hematopoietic marker expression in npas 4 l mutants independently of lmo2 (Dooley et al., 2005). These observations as well as an additional study on a hypomorphic zebrafish tal1 allele (Juarez et al., 2005) suggest different functions of Tal1 during early endothelial and hematopoietic specification compared to later stages. Early stages may be independent of Lmo2 function (Dooley et al., 2005). Of further interest in this context is that mouse mutants carrying a Tal1 allele with a dysfunctional DNA-binding site encoding sequence but intact binding site for Lmo2 have been found to still be a potent oncogene, suggesting that late roles of TAL1 may not even require its direct binding to the DNA (Draheim et al., 2011).

Most literature on zebrafish tal1 and mammalian Tal1 orthologues suggests generally conserved functionality of these factors. One indirect exception is that one of the downstream effectors of zebrafish Tal1, the helix-loophelix transcription factor Hey2, appears to act upstream of Notch during hematopoietic stem cell development instead of downstream of it as published in mouse studies (Rowlinson and Gering, 2010). 


\subsubsection{Lmo2}

LMO2 is a LIM domain containing transcription factor required for hematopoietic development. Both zebrafish and mouse Lmo2 loss-of-function models exhibit defective erythroid development and marker gene expression (Patterson et al., 2007; Warren et al., 1994). Zebrafish lmo2 mutants also display vascular defects that are linked to reduced endothelial cell migration (Matrone et al., 2017; Weiss et al., 2012). LMO2 is part of transcription factor complexes involving TAL1 (Warren et al., 1994; Osada et al., 1995), and has been reported to function as part of a scaffold for these transcription factor complexes (El Omari et al., 2011).

Although Lmo2 function is often studied as a part of a transcription factor complex involving Tal1 (Gering et al., 2003; Patterson et al., 2007; Draheim et al., 2011), some studies suggest that Tal1 acts independently of Lmo2 in certain contexts (Dooley et al., 2005; Juarez et al., 2005). A more detailed discussion of these differences can be found in section 1.3.3.

\subsubsection{Other early endothelial factors}

As the work described in this thesis focuses on Npas4l and three of its direct targets etsrp, tal1 and lmo2, the introduction necessarily focuses on these genes. But this does not mean, that these genes are the only transcription factors that are important in early vascular development. Other early acting transcription factors play important and often partially redundant roles in hematovascular development as well. However, in many cases these effects appear to be less clean than in mutants of the transcription factor genes described above.

Two well-described examples for such genes are the ETS transcription factors fli1a and fli1b. The initial expression of fli1a can be observed in cloche mutants, meaning that the initiation of it is independent of Npas4l transcriptional activity (Brown et al., 2000). While morpholino knockdowns of fli1a cause vascular defects (Liu et al., 2008), knockouts of each of these genes individually do not cause vascular defects visible at the physiological level (Craig et al., 2015). Endothelial marker expression in both of these mutants was impaired, though (Craig et al., 2015). In mouse, Fli1a expression is induced by ETV2 transcriptional activity and maintains the expression of genes required for endothelial cell development and survival when Etv2 is not expressed anymore (Abedin et al., 2014). 
Other examples of early endothelial transcription factors that have been placed at or close to the top of the endothelial hierarchy are hhex (Liao et al., 2000), foxc1a/foxc1b (De Val et al., 2008; Veldman and Lin, 2012), aggf1 (Li et al., 2014) and gata2 (Dobrzycki et al., 2020), but all of their mutants have milder defects compared to npas $4 l$ mutants. 



\title{
Chapter 2
}

\begin{abstract}
Aims
Homozygous mutations in the npas $4 l$ gene cause the strongest vascular phenotype known in vertebrates. However, since the gene was only recently discovered (Reischauer et al., 2016), little is known about the dynamics of its expression. Therefore, I started the project with three aims to generate basic tools for the investigation of npas\&l. I planned to:
\end{abstract}

1. generate a tagged Npas4l-V5 line for protein detection and proteomic analyses.

2. visualize npas 4 l expression in vivo by generating a fluorescent reporter line.

3. characterize the expression pattern and protein localization.

When initial results indicated that the aims mentioned above would be obtainable, I added two aims, to answer the following biological questions using the newly generated tools:

4. What happens to npas $4 l$ expressing cells in npas 4 l mutants?

5. How does the npas 4 l mutant phenotype differ from the mutant phenotypes of its direct downstream targets etsrp, tal1 and $\operatorname{lmo} 2 ?$ 



\section{Chapter 3}

\section{Materials and methods}

\subsection{Materials}

\subsubsection{Organisms}

\subsubsection{Bacterial strain}

E. coli strain DH5 $\alpha$ was used for all transformations and plasmid amplification.

\subsubsection{Established zebrafish lines used in this study}

Table 3.1: Established zebrafish lines used in this thesis.

\begin{tabular}{l|l|l} 
Allele & Description & Reference \\
\hline cloche $^{\text {m39 }}$ & $\begin{array}{l}\text { large deletion } \\
\text { mutant } \\
\text { including npas4l }\end{array}$ & (Stainier et al., 1995) \\
\hline npas4 $l^{\text {bns297 }}$ & $\begin{array}{l}\text { small } \\
\text { out-of-frame } \\
\text { indel in the } \\
\text { bHLH domain }\end{array}$ & \\
\hline$T g(U A S: G F P)^{\text {nkuasgfp1a }}$ & $\begin{array}{l}\text { GFP reporter } \\
\text { for Gal4 driver }\end{array}$ & (Asakawa et al., 2008) \\
& lines \\
\hline$T g(U A S: n f s B-$ & $\begin{array}{l}\text { NTR-mCherry } \\
\text { fusion reporter }\end{array}$ & (Davison et al., 2007) \\
$m C h)^{c 264}$ & for Gal4 driver & \\
& lines \\
\hline
\end{tabular}


Table 3.1: Established zebrafish lines used in this thesis.

\begin{tabular}{l|l|l} 
Allele & Description & Reference \\
\hline Tg(fli1a:GFP $)^{y 1}$ & $\begin{array}{l}\text { early expressed } \\
\text { pan-endothelial } \\
\text { reporter line }\end{array}$ & (Lawson and Weinstein, 2002) \\
\hline Tg(fli1a:nls-GFP)y7 & $\begin{array}{l}\text { early expressed } \\
\text { nuclear } \\
\text { pan-endothelial } \\
\text { reporter line }\end{array}$ & \\
\hline Tg(kdrl:TagBFP)mu293 & $\begin{array}{l}\text { (Roman et al., 2002) } \\
\text { endothelial }\end{array}$ & \\
\hline Tg(tnnc2:nls- & reporter line & \\
TdTomato) & immature & (Tadesse Tsedeke et al., \\
& cardiomyocyte & manuscript in preparation) \\
marker &
\end{tabular}

\subsubsection{Self-generated zebrafish lines}

Table 3.2: Self-generated zebrafish lines

\begin{tabular}{|c|c|}
\hline Allele & Description \\
\hline npas4l $\mathrm{l}^{\text {Pt(npas4l-V5)bn314 }}$ & $\begin{array}{l}\text { V5-tagged allele for protein } \\
\text { detection of Npas4l }\end{array}$ \\
\hline npas4l $l^{\text {Pt }}{ }^{\left.\text {npas } 4 l-p 2 a-G a l_{4}-V P 16\right) b n s 313}$ & $\begin{array}{l}\text { endogenous Gal4-VP16 knock-in } \\
\text { reporter allele with intact Npas } 41\end{array}$ \\
\hline$n p a s 4 l^{\text {Pt }\left(+36 b p-n p a s 4 l-p 2 a-G a l_{4}-V P 16\right) b n s 423}$ & $\begin{array}{l}\text { endogenous Gal4-VP16 knock-in } \\
\text { reporter allele with mutated } \\
\text { Npas4l }\end{array}$ \\
\hline$e^{e t s r p} p^{\text {bns422 }}$ & -4 bp out-of-frame mutant allele \\
\hline$e^{e t s r p}{ }^{b n s 426}$ & -9 bp in-frame mutant allele \\
\hline tal1 $1^{\text {bns497 }}$ & -7 bp out-of-frame mutant allele \\
\hline tal1 $1^{\text {bns } 498}$ & -6 bp in-frame mutant allele \\
\hline $\operatorname{lmo} 2^{b n s 499}$ & +2 bp out-of-frame mutant allele \\
\hline $\operatorname{lmo} 2^{b n s 500}$ & -6 bp in-frame mutant allele \\
\hline$i l 11 r a^{P t\left(i l 11 r a-p 2 a-G a l_{4}-V P 16\right) b n s 424}$ & $\begin{array}{l}\text { endogenous Gal4-VP16 knock-in } \\
\text { reporter allele with intact Il11ra }\end{array}$ \\
\hline $\operatorname{Tg}(U A S: m T a g B F P \mathscr{Q})^{b n s 425}$ & $\begin{array}{l}\text { transgenic reporter line to } \\
\text { visualize Gal4 expression }\end{array}$ \\
\hline notch1 $a^{P t(\text { notch } 1 a-V 5) N N-107}$ & $\begin{array}{l}\text { V5-tagged allele for protein } \\
\text { detection of Notch1a }\end{array}$ \\
\hline
\end{tabular}




\begin{tabular}{l|l}
\hline notch $3^{\text {Pt(notch3-V5) }}$ & $\begin{array}{l}\text { V5-tagged allele for protein } \\
\text { detection of Notch3 }\end{array}$ \\
\hline$p d g f a b^{\text {bns322 }}$ (promoter deletion) & bp out-of-frame mutant allele \\
\hline$p d g f b a^{\text {bns323 }}$ & -10 bp out-of-frame mutant allele \\
\hline$p d g f b b^{\text {bns324 }}$ & -26 bp out-of-frame mutant allele \\
\hline$p d g f c^{\text {bns325 }}$ & -5 bp out-of-frame mutant allele \\
\hline$p d g f d^{\text {bns326 }}$ & -11 bp out-of-frame mutant allele
\end{tabular}

\subsubsection{Zebrafish feeding}

Fish were fed with SDS (Special Diets Services) dry food from 5 days post fertilization onwards supplemented with life brine shrimp (Artemia salinaris) beginning at $12 \mathrm{dpf}$. Until about $14 \mathrm{dpf}$, SDS 100 was fed, SDS 200 from about $21 \mathrm{dpf}$ and SDS 400 in adult stages. These stages only represent guidelines and final feeding decisions were adjusted to the body size of the larvae or fish.

\subsubsection{Laboratory equipment}

Table 3.3: List of Laboratory equipment

\begin{tabular}{l|l|l} 
Model & Manufacturer & Description \\
\hline $\begin{array}{l}\text { Bullet Blender } \\
\text { BB24gold }\end{array}$ & NextAdvance & tissue homogenization \\
\hline $\begin{array}{l}\text { 220GX.0 } \\
\text { Explorer }\end{array}$ & Ohaus & fine balance, 1 mg accuracy \\
\hline AZ1502 & Sartorius & balance accuracy \\
\hline $\begin{array}{l}\text { Cell Observer } \\
\text { SD }\end{array}$ & ZEISS & spinning disc microscope \\
\hline CFX connect & Bio Rad & $\begin{array}{l}\text { CFX connect real time PCR } \\
\text { detection system }\end{array}$ \\
\hline CL-S631 & Citizen & label printer \\
\hline CM1950 & Leica & manual cryotome \\
\hline CM3050S & Leica & $\begin{array}{l}\text { semi-automatic cryotome with foot } \\
\text { pedal }\end{array}$ \\
\hline Dumont size & FST by & fine forceps \\
55 & Dumont & real-time PCR system for high \\
\hline Eco & Illumina & resolution melt analysis \\
\hline
\end{tabular}


Table 3.3: List of Laboratory equipment

\begin{tabular}{|c|c|c|}
\hline Model & Manufacturer & Description \\
\hline $\begin{array}{l}\text { G560E Vortex } \\
\text { Genie } 2\end{array}$ & $\begin{array}{l}\text { Scientific } \\
\text { Insdustries }\end{array}$ & shaker ("vortexer") \\
\hline $\begin{array}{l}\text { Gel Doc EZ } \\
\text { system }\end{array}$ & Bio Rad & gel imager system \\
\hline HB-10 UVP & analytic Jena & hybridizer oven \\
\hline HMT75M421 & Bosh & microwave \\
\hline Incu-line & VWR & heating units /incubators \\
\hline $\begin{array}{l}\text { LSM800 } \\
\text { Observer }\end{array}$ & ZEISS & confocal micoscope with airyscan \\
\hline $\begin{array}{l}\text { mastercycler } \\
\text { Pro }\end{array}$ & Eppendorf & DNA amplification (PCR) \\
\hline $\begin{array}{l}\text { MiniStar } \\
1205-0713\end{array}$ & VWR & small centrifuge \\
\hline MIR-254-PE & Panasonic & zebrafish incubator \\
\hline $\begin{array}{l}\text { Model } \\
40-07-08\end{array}$ & PeqLab & gel chamber, small size \\
\hline $\begin{array}{l}\text { Model } \\
40-09-11\end{array}$ & PeqLab & gel chamber, medium size \\
\hline $\begin{array}{l}\text { Model } \\
40-15-15\end{array}$ & PeqLab & gel chamber, large size \\
\hline $\begin{array}{l}\text { Model } \\
460-3267\end{array}$ & VWR & heating block \\
\hline Model 5424 & Eppendorf & $\begin{array}{l}\text { centrifuge for } 1.5-2.0 \mathrm{ml} \text { tubes, fixed } \\
\text { rotor, uncooled }\end{array}$ \\
\hline Model 5424R & Eppendorf & $\begin{array}{l}\text { centrifuge for } 1.5-2.0 \mathrm{ml} \text { tubes, fixed } \\
\text { rotor, cooled }\end{array}$ \\
\hline Model 5810RF & Eppendorf & $\begin{array}{l}\text { centrifuge for } 15 \text { and } 50 \mathrm{ml} \text { tubes, } \\
\text { swinging rotor, cooled }\end{array}$ \\
\hline Model E0303 & PeqLab & electrophoresis power supply \\
\hline Model T6 & Heraeus & bacterial incubator \\
\hline $\begin{array}{l}\text { Multipette } \\
\text { E3x }\end{array}$ & Eppendorf & semi-automatic dispenser \\
\hline $\begin{array}{l}\text { Multitron } \\
\text { Standard }\end{array}$ & Infors HAT & bacterial incubator shaker \\
\hline $\begin{array}{l}\text { Nanodrop } \\
2000 \mathrm{c}\end{array}$ & $\begin{array}{l}\text { Thermo Fisher } \\
\text { Scientific }\end{array}$ & $\begin{array}{l}\text { DNA concentration and quality } \\
\text { analysis }\end{array}$ \\
\hline
\end{tabular}


Table 3.3: List of Laboratory equipment

\begin{tabular}{|c|c|c|}
\hline Model & Manufacturer & Description \\
\hline $\begin{array}{l}\text { NextSeq500 } \\
\text { instrument }\end{array}$ & Illumina & next generation sequencing \\
\hline P-1000 & $\begin{array}{l}\text { Sutter } \\
\text { instruments }\end{array}$ & micropipette puller \\
\hline P95 & Mitsubishi & printer for gel images \\
\hline Pipetboy2 & Integra & pipettor for serological pipettes \\
\hline $\begin{array}{l}\text { Pipetman } \\
\text { comfort, } 10 \mathrm{ml}\end{array}$ & Gilson & pipette \\
\hline $\begin{array}{l}\text { Pipetman } \\
\text { comfort, } \\
1000 \mathrm{\mu l}\end{array}$ & Gilson & pipette \\
\hline $\begin{array}{l}\text { Pipetman } \\
\text { comfort, } 2 \mu l\end{array}$ & Gilson & pipette \\
\hline $\begin{array}{l}\text { Pipetman } \\
\text { comfort, } 20 \mu l\end{array}$ & Gilson & pipette \\
\hline $\begin{array}{l}\text { Pipetman } \\
\text { comfort, } 200 \mu l\end{array}$ & Gilson & pipette \\
\hline PV876WPI & $\begin{array}{l}\text { World } \\
\text { precision } \\
\text { instruments }\end{array}$ & $\begin{array}{l}\text { pneumatic pico pump for } \\
\text { microinjections }\end{array}$ \\
\hline SMZ18 & Nikon & $\begin{array}{l}\text { stereomicroscope for screening and } \\
\text { dissection }\end{array}$ \\
\hline SMZ25 & Nikon & $\begin{array}{l}\text { stereomicroscope for screening and } \\
\text { documentation }\end{array}$ \\
\hline $\begin{array}{l}\text { SOLA light } \\
\text { engine }\end{array}$ & Lumencor & LED laser unit for stereomicroscopes \\
\hline Stemi2000 & ZEISS & stereomicroscope for dissection \\
\hline $\begin{array}{l}\text { Sunflower mini } \\
\text { shaker PS-3D }\end{array}$ & BioSan & mini shaker \\
\hline Z1 & ZEISS & lightsheet microscope \\
\hline $\begin{array}{l}\text { Zebrafish aqua } \\
\text { culture system }\end{array}$ & Tecniplast & zebrafish \\
\hline
\end{tabular}




\subsubsection{Laboratory consumables}

Table 3.4: List of lab consumables

\begin{tabular}{|c|c|c|}
\hline $\begin{array}{l}\text { Product \# or } \\
\text { model }\end{array}$ & Supplier & Description \\
\hline 72.706 & Sarstedt & micro tubes, $1.5 \mathrm{ml}$ \\
\hline 304000 & B.Braun & $\begin{array}{l}\text { sterican cannula, } \\
0.3 \times 13 \mathrm{~mm}\end{array}$ \\
\hline 5242956.003 & Eppendorf & microloader tips, $20 \mu \mathrm{l}$ \\
\hline$\mu$-dish & Ibidi & $\begin{array}{l}\text { glass bottom dish, } 35 \\
\text { mm }\end{array}$ \\
\hline $188-271$ & Greiner Bio-one & $15 \mathrm{ml}$ tubes \\
\hline $227-261$ & Greiner Bio-one & $50 \mathrm{ml}$ tubes \\
\hline $24 \times 50 \mathrm{~mm} \mathrm{\# 1}$ & Menzel-Gläser & deckgläser \\
\hline $3-1011$ & Neolab & $\begin{array}{l}\text { Parafilm "M" } \\
\text { laboratory film }\end{array}$ \\
\hline $606-160$ & Greiner Bio-one & $\begin{array}{l}\text { serological pipettes, } \\
5 \mathrm{ml}\end{array}$ \\
\hline 62.515 .006 & Sarstedt & bacterial culture tube \\
\hline $627-102$ & Greiner Bio-one & Petridishes $35 / 10$ \\
\hline 639-161 & Greiner Bio-one & Petridishes 145/20 \\
\hline 72.695 .500 & Sarstedt & micro tubes, $2.0 \mathrm{ml}$ \\
\hline 72.985 .002 & Sarstedt & PCR strips, $8 \times 0.2 \mathrm{ml}$ \\
\hline $739-288$ & Greiner Bio-one & pipette tips, $200 \mu l$ \\
\hline $740-288$ & Greiner Bio-one & pipette tips, $1000 \mu l$ \\
\hline $760-107$ & Greiner Bio-one & $\begin{array}{l}\text { serological pipettes, } \\
25 \mathrm{ml}\end{array}$ \\
\hline $771-288$ & Greiner Bio-one & pipette tips, $10 \mu l$ \\
\hline $774-288$ & Greiner Bio-one & pipette tips, $20 \mu \mathrm{l}$ \\
\hline 86.1254 .001 & Sarstedt & $\begin{array}{l}\text { serological pipettes, } \\
10 \mathrm{ml}\end{array}$ \\
\hline BBBB523 & B.Braun & scalpel, blade no. 23 \\
\hline PC57.1 & Carl Roth & $\begin{array}{l}\text { petridish spatula, } \\
\text { L-shaped }\end{array}$ \\
\hline SG-C-L & StarGuard & nitrile gloves, large \\
\hline
\end{tabular}




\subsubsection{Chemical reagents and macromolecules}

\subsubsection{Antibiotics}

\begin{tabular}{l|l|l|l} 
Antibiotic & Concentration & Supplier & Catalog \# \\
\hline Ampicillin & $100 \mu \mathrm{g} / \mathrm{mL}$ & Merck & 171254-25GM \\
\hline Kanamycin & $50 \mu \mathrm{g} / \mathrm{mL}$ & Merck & $420311-25 \mathrm{GM}$ \\
\hline Streptomycin & $200 \mu \mathrm{g} / \mathrm{ml}$ & Merck & 5711-100GM
\end{tabular}

\subsubsection{Antibodies}

Table 3.6: List of antibodies

\begin{tabular}{|c|c|c|c|c|}
\hline Antibody & $\begin{array}{l}\text { Species } \\
\text { and type }\end{array}$ & Dilution & Manufactur & $\begin{array}{l}\text { Catalog } \\
\#\end{array}$ \\
\hline Anti-EGFP & $\begin{array}{l}\text { Chicken } \\
\text { polyclonal }\end{array}$ & $1: 500$ & Aves Lab & gfp-1020 \\
\hline $\begin{array}{l}\text { Living Colors } \\
\text { DsRed }\end{array}$ & $\begin{array}{l}\text { Rabbit } \\
\text { polyclonal }\end{array}$ & $1: 500$ & Takara & 632496 \\
\hline $\begin{array}{l}\text { V5 Tag } \\
\text { Monoclonal } \\
\text { Antibody }\end{array}$ & $\begin{array}{l}\text { Mouse } \\
\text { IgG2a }\end{array}$ & $1: 250$ & $\begin{array}{l}\text { Thermo } \\
\text { Fisher } \\
\text { Scientific }\end{array}$ & R960-25 \\
\hline $\begin{array}{l}\text { Anti-Pax2a } \\
\text { antibody }\end{array}$ & $\begin{array}{l}\text { Rabbit } \\
\text { polyclonal }\end{array}$ & $1: 250$ & GeneTex & GTX128127 \\
\hline $\begin{array}{l}\text { Alexa Fluor } \\
488 \\
\text { anti-Chicken }\end{array}$ & Goat IgG & $1: 500$ & $\begin{array}{l}\text { Life } \\
\text { Technologies }\end{array}$ & A11039 \\
\hline $\begin{array}{l}\text { Alexa Fluor } \\
565 \\
\text { anti-Rabbit }\end{array}$ & Goat IgG & $1: 500$ & $\begin{array}{l}\text { Life } \\
\text { Technologies }\end{array}$ & A27039 \\
\hline $\begin{array}{l}\text { Alexa Fluor } \\
647 \text { anti-Mouse }\end{array}$ & Goat IgG & $1: 500$ & $\begin{array}{l}\text { Life } \\
\text { Technologies }\end{array}$ & A21236 \\
\hline Anti-DIG & $\begin{array}{l}\text { sheep Fab } \\
\text { fragments }\end{array}$ & $1: 5000$ & Roche & 11093274910 \\
\hline
\end{tabular}




\subsubsection{Buffers}

Table 3.7: Lift of buffers and solutions

\begin{tabular}{|c|c|}
\hline Buffer & Recipe \\
\hline $\begin{array}{l}\text { permeabilization } \\
\text { buffer }\end{array}$ & $0.5 \%$ Triton X-100 dissolved in $1 \mathrm{X}$ PBS \\
\hline 10X PBS & 10 PBS tablets (Merck) ad $2 \mathrm{~L} \mathrm{H}_{2} \mathrm{O}$ \\
\hline 10X TBE & $\begin{array}{l}121 \mathrm{~g} \text { Tris }+62 \mathrm{~g} \mathrm{H}_{3} \mathrm{BO}_{3}+7.4 \mathrm{~g} \text { EDTA ad } 1 \mathrm{~L} \\
\mathrm{H}_{2} \mathrm{O}\end{array}$ \\
\hline 20X SSC & $\begin{array}{l}175.3 \mathrm{~g} \mathrm{NaCl}+88.2 \mathrm{~g} \mathrm{Na}{ }_{3} \mathrm{C}_{6} \mathrm{H}_{5} \mathrm{O}_{7}, \text { adjusted to } \\
\text { pH } 7 \text { in } 800 \mathrm{ml} \mathrm{H}_{2} \mathrm{O} \text {, then ad } 1000 \mathrm{ml} \mathrm{H}_{2} \mathrm{O}\end{array}$ \\
\hline $\begin{array}{l}\text { Alkaline Tris } \\
\text { buffer }\end{array}$ & $\begin{array}{l}100 \mathrm{mM} \text { Tris- } \mathrm{HCl} \text { pH } 9.5+100 \mathrm{mM} \mathrm{NaCl}+0.1 \% \\
\text { Tween } 20 \text { in } \mathrm{H}_{2} \mathrm{O}\end{array}$ \\
\hline $\begin{array}{l}\text { Blocking buffer } \\
\text { (immunostaining) }\end{array}$ & $1 \mathrm{X}$ PBST $+5 \%$ goat serum \\
\hline $\begin{array}{l}\text { Blocking buffer (in } \\
\text { situ) }\end{array}$ & $2 \mathrm{mg} / \mathrm{mL}$ BSA $+2 \%$ sheep serum in $0.1 \%$ PBST \\
\hline Egg water & $3 \mathrm{~g}$ Instant Ocean $+0.75 \mathrm{~g} \mathrm{CaSO}_{4}$ ad $10 \mathrm{~L} \mathrm{H}_{2} \mathrm{O}$ \\
\hline $\begin{array}{l}\text { HM (-) for in situ } \\
\text { hybridization }\end{array}$ & $\begin{array}{l}50 \% \text { formamide }+5 \mathrm{X} \text { SSC }+0.1 \% \text { Tween } 20 \text {, } \\
\text { adjusted to pH } 6 \text { with } 1 \mathrm{M} \mathrm{C}_{6} \mathrm{H}_{8} \mathrm{O}_{7}\end{array}$ \\
\hline $\begin{array}{l}\mathrm{HM}(+) \text { for in situ } \\
\text { hybridization }\end{array}$ & HM $(-)+$ Heparin $50 \mu \mathrm{g} / \mathrm{mL}+$ tRNA $500 \mu \mathrm{g} / \mathrm{mL}$ \\
\hline PBST & PBS $+0.1 \%$ Triton $\mathrm{X}-100$ \\
\hline PBSTween & PBS $+0.1 \%$ Tween-20 \\
\hline $1 \mathrm{M}$ Tris- $\mathrm{HCl}$ & $\begin{array}{l}1 \mathrm{~mol} \text { Tris base in } 800 \mathrm{ml} \mathrm{H}_{2} \mathrm{O} \text {, adjust to } \mathrm{pH} 8 \text {, ad } \\
1000 \mathrm{ml} \mathrm{H}_{2} \mathrm{O}\end{array}$ \\
\hline
\end{tabular}

\subsubsection{Chemicals}

Table 3.8: List of chemicals

\begin{tabular}{l|l|l} 
Chemical & Supplier & $\begin{array}{l}\text { Catalog } \\
\#\end{array}$ \\
\hline 10X NEBuffer 2.1 & NEB & B7202S \\
\hline 10X NEBuffer 3.1 & NEB & B7203S \\
\hline 2-propanol & Carl Roth & 9866.4 \\
\hline agarose & Merck & A9539 \\
\hline $\begin{array}{l}\text { agarose, low gelling tempera- } \\
\text { ture }\end{array}$ & Merck & A9414 \\
\hline BM Purple AP substrate & Roche & 10165921001 \\
\hline
\end{tabular}


Table 3.8: List of chemicals

\begin{tabular}{|c|c|c|}
\hline Chemical & Supplier & $\begin{array}{l}\text { Catalog } \\
\#\end{array}$ \\
\hline chloroform & Merck & $\mathrm{C} 2432$ \\
\hline citric acid & Merck & 251275 \\
\hline CutSmart buffer & NEB & B7204S \\
\hline dimethylsulfoxide & Merck & D8418 \\
\hline DNA Gel loading dye $(6 \mathrm{x})$ & Thermo Fisher Scientific & R0611 \\
\hline ethanol & Carl Roth & P075.4 \\
\hline GeneRuler $1 \mathrm{~kb}$ ladder & Thermo Fisher Scientific & SM0314 \\
\hline GeneRuler 100 bp ladder) & Thermo Fisher Scientific & SM0241 \\
\hline glycerol & Carl Roth & 7533.1 \\
\hline heparin & Merck & H6279 \\
\hline hydrochloridic acid & Merck & H1758 \\
\hline $\begin{array}{lll}\text { hydrogen peroxide solution, } \\
3 \%\end{array}$ & Merck & 88597 \\
\hline methanol & Carl Roth & KK39.2 \\
\hline methylene blue & Merck & A514.1 \\
\hline mineral oil & Merck & M5904 \\
\hline normal goat serum & Merck & 566380 \\
\hline N-phenylthiourea (PTU) & Merck & P7629 \\
\hline nuclease-free water & Ambion & AM9938 \\
\hline O.C.T tissue TEK & Sakura & 4583 \\
\hline paraformaldehyde & Merck & 158127 \\
\hline phenol red solution & Merck & P3532 \\
\hline phosphate-buffered saline & Merck & $\mathrm{P} 4417$ \\
\hline pronase & Roche & 53702 \\
\hline proteinase $\mathrm{K}$ & Roche & 1.24568 \\
\hline sodium citrate & Merck & 71498 \\
\hline sodium hydroxide & Merck & 221465 \\
\hline sucrose & Merck & 1.07687 \\
\hline SYBR Safe & Thermo Fisher Scientific & S33102 \\
\hline tricaine & Merck & A5040 \\
\hline Tris & Merck & 648310 \\
\hline Triton X-100 & Merck & T8787 \\
\hline Trizol & Ambion & 15596018 \\
\hline tRNA & Merck & $\mathrm{R} 8759$ \\
\hline
\end{tabular}




\subsubsection{Enzymes}

Table 3.9: List of enzymes

\begin{tabular}{l|l|l} 
Enzyme & Supplier & Catalog \# \\
\hline T4 DNA ligase & Takara & 2011A \\
\hline BamHI-HF & NEB & R3136S \\
\hline EcoRI-HF & NEB & R3101S \\
\hline XhoI & NEB & R0146S \\
\hline KAPA2G fast ReadyMix & $\begin{array}{l}\text { Kapa } \\
\text { Biosystem }\end{array}$ & KK5101 \\
\hline $\begin{array}{l}\text { PrimeSTAR max DNA } \\
\text { polymerase }\end{array}$ & Takara & R045A \\
\hline $\begin{array}{l}\text { DyNAmo ColorFlash SYBR } \\
\text { Green qPCR Mix }\end{array}$ & $\begin{array}{l}\text { Thermo Fisher } \\
\text { Scientific }\end{array}$ & F416L \\
\hline DNase I & Qiagen & 79254 \\
\hline T7 RNA polymerase & Promega & P2075 \\
\hline T7 EndonucleaseI & NEB & M0302S \\
\hline RNasin ribonuclease inhibitor & Promega & N2511 \\
\hline BfuAI & NEB & R0701S \\
\hline BsaI & NEB & R0535 \\
\hline BspQI & NEB & R0712S \\
\hline NotI HF & NEB & R3189S
\end{tabular}

\subsubsection{Growth media}

Table 3.10: List of Growth media components

\begin{tabular}{l|l|l} 
Product & Supplier & Catalog \# \\
\hline $\begin{array}{l}\text { LB-agar } \\
\text { (Luria/Miller) }\end{array}$ & Carl Roth & R8759 \\
\hline $\begin{array}{l}\text { LB-medium } \\
\text { (Luria/Miller) }\end{array}$ & Carl Roth & 6673.4 \\
\hline DMEM/F12 & Thermo Fisher Scientific & 11320033
\end{tabular}

\subsubsection{Kits}

\begin{tabular}{l|l|l} 
Kit & Supplier & Catalog \# \\
\hline GeneJET gel extraction kit & $\begin{array}{l}\text { Thermo Fisher Sci- } \\
\text { entific }\end{array}$ & K0691 \\
\hline
\end{tabular}




\begin{tabular}{l|l|l} 
Kit & Supplier & Catalog \# \\
\hline GeneJET PCR purification kit & $\begin{array}{l}\text { Thermo Fisher Sci- } \\
\text { entific }\end{array}$ & K0701 \\
\hline GeneJET plasmid miniprep kit & $\begin{array}{l}\text { Thermo Fisher Sci- } \\
\text { entific }\end{array}$ & K0502 \\
\hline Maxima & $\begin{array}{l}\text { Thermo Fisher Sci- } \\
\text { entific }\end{array}$ & K1672 \\
\hline MEGAshortscript T7 kit & Ambion & AM1354 \\
\hline miRNeasy micro kit & Qiagen & 217084 \\
\hline $\begin{array}{l}\text { mMessage mMACHINE SP6 } \\
\text { transcription kit }\end{array}$ & Ambion & AM1340 \\
\hline $\begin{array}{l}\text { mMessage mMACHINE T3 } \\
\text { transcription kit }\end{array}$ & Ambion & AM1348 \\
\hline $\begin{array}{l}\text { mMessage mMACHINE T7 } \\
\text { transcription kit }\end{array}$ & Ambion & AM1344 \\
\hline pGEM-T-easy vector kit & Promega & A1360 \\
\hline QiaQuick nucleotide removal kit & Qiagen & 28304 \\
\hline QiaPrep spin miniprep kit & 27104 & R1016 \\
\hline RNA clean and concentrator & Zymo Research & \\
\hline
\end{tabular}

\subsubsection{Oligonucleotides}

Synthetic guide RNA's were used to generate the knock-ins for npas $4 l$ and il11ra. Codon-optimized mTagBFP2 was ordered as a synthesized GeneStrand. These reagents are not included in this list and can be found in section 3.1.5. All other oligonucleotides were ordered as custom made oligos in tubes. V5-HDR donors were ordered as PAGE or HPLC-purified reagents to reduce toxicity of the injections. For all other application, desalted oligos were ordered and used.

Table 3.12: Oligonucleotides used in this thesis.

\begin{tabular}{l|l}
$\mathbf{5}$ ' to $\mathbf{3}$ ' nucleotide sequence & $\begin{array}{l}\text { Primer name and } \\
\text { description }\end{array}$ \\
\hline $\begin{array}{l}\text { GCAGAATTCGCCACCATGGAAATG } \\
\text { TACCAATCTGGATTTTACAC }\end{array}$ & etsrp pCS2z cloning fwd \\
\hline $\begin{array}{l}\text { TGCCTCGAGCTAATGTGTCCAGGA } \\
\text { CTCTGTGG }\end{array}$ & etsrp pCS2z cloning rev \\
\hline CGAGGTTCTGGTAGGTTTGAG & $\begin{array}{l}\text { etsrp qPCR fwd } \\
\text { (Reischauer et al., 2016) }\end{array}$ \\
\hline GCACAAAGGTCATGTTCTCAC & $\begin{array}{l}\text { etsrp qPCR rev } \\
\text { (Reischauer et al., 2016) }\end{array}$ \\
\hline
\end{tabular}


Table 3.12: Oligonucleotides used in this thesis.

5' to 3' nucleotide sequence

Primer name and description

\begin{tabular}{|c|c|}
\hline AAACAGCCATCACCAGTCCAACTT & etsrp sgRNA1 (antisense) \\
\hline TAGGAAGTTGGACTGGTGATGGCT & etsrp sgRNA1 (sense) \\
\hline $\begin{array}{l}\text { TTTCTGCTAGAACTCCTGCTGGAT } \\
\text { TC }\end{array}$ & etsrp sgRNA1 HRMA fwd \\
\hline CCTCAGCGGGATCTGACAT & etsrp sgRNA1 HRMA rev \\
\hline ACGCCAATGTCCGCCTAC & $\begin{array}{l}\text { etv2 mutation flanking } \\
\text { PCR fwd }\end{array}$ \\
\hline $\begin{array}{l}\text { CTATTTGTCCACTACCCAAGTGTT } \\
\text { G }\end{array}$ & $\begin{array}{l}\text { etv2 mutation flanking } \\
\text { PCR rev }\end{array}$ \\
\hline $\begin{array}{l}\text { CATTAACCCTCACTAAAGGGAATC } \\
\text { CTCTGAGCCTTCTCGTTG }\end{array}$ & gata1a ISH probe fwd \\
\hline $\begin{array}{l}\text { TAATACGACTCACTATAGGGCTGG } \\
\text { CCGTTCATCTTATGGT }\end{array}$ & gata1a ISH probe rev \\
\hline $\begin{array}{l}\text { CGGCCCACCTGTAGACTTGTCTGT } \\
\text { TGTCCTCACTGGATAGGACTGAAG } \\
\text { TATGTA }\end{array}$ & $\begin{array}{l}\text { il11ra Gal4 3' homology } \\
\text { arm, antisense }\end{array}$ \\
\hline $\begin{array}{l}\text { AAGTACATACTTCAGTCCTATCCA } \\
\text { GTGAGGACAACAGACAAGTCTACA } \\
\text { GGTGGG }\end{array}$ & $\begin{array}{l}\text { il11ra Gal4 3' homology } \\
\text { arm, sense }\end{array}$ \\
\hline $\begin{array}{l}\text { ATCCATCCGGTTCCTCTGTTGGCT } \\
\text { CTTTTGGAGGTTCTAAAATTGCAA } \\
\text { AACAGGG }\end{array}$ & $\begin{array}{l}\text { il11ra Gal4 5' homology } \\
\text { arm, antisense }\end{array}$ \\
\hline $\begin{array}{l}\text { GCGGCCCTGTTTTGCAATTTTAGA } \\
\text { ACCTCCAAAAGAGCCAACAGAGGA } \\
\text { ACCGGAT }\end{array}$ & $\begin{array}{l}\text { il11ra Gal4 5' homology } \\
\text { arm, sense }\end{array}$ \\
\hline CCTCCAAAAGAGCCAACAGAG & $\begin{array}{l}\text { il11ra sgRNA7 HRMA } \\
\text { fwd }\end{array}$ \\
\hline TGTAGACTTGTCTGTTGTCCTCA & il11ra sgRNA7 HRMA rev \\
\hline TGCTCACACACAATGTCCGAG & $\begin{array}{l}\text { lmo2 mutation flanking } \\
\text { PCR fwd }\end{array}$ \\
\hline CTGCCGTTGAGTTTGGTCCA & $\begin{array}{l}\text { lmo2 mutation flanking } \\
\text { PCR fwd }\end{array}$ \\
\hline $\begin{array}{l}\text { GCAGAATTCGCCACCATGGCTTCT } \\
\text { ACAATTGAACGGAAAACAC }\end{array}$ & lmo2 pCS2z cloning fwd \\
\hline $\begin{array}{l}\text { TGCCTCGAGCTATACTATGCTGCC } \\
\text { GTTGAGTTTGG }\end{array}$ & lmo2 pCS2z cloning rev \\
\hline
\end{tabular}


Table 3.12: Oligonucleotides used in this thesis.

\begin{tabular}{|c|c|}
\hline $5^{\prime}$ to $3^{\prime}$ nucleotide sequence & $\begin{array}{l}\text { Primer name and } \\
\text { description }\end{array}$ \\
\hline ACTGGTTCCTCTGAAGGGTC & $\begin{array}{l}\text { Lmo2 qPCR fwd } \\
\text { (Reischauer et al., 2016) }\end{array}$ \\
\hline TCTTTGGCGTTTGAAGGGAG & $\begin{array}{l}\text { Lmo2 qPCR rev } \\
\text { (Reischauer et al., 2016) }\end{array}$ \\
\hline AAACGGATCCTCTTTTCACAGGAA & lmo2 sgRNA4 (antisense) \\
\hline TAGGTTCCTGTGAAAAGAGGATCC & lmo2 sgRNA4 (sense) \\
\hline ACTGTTTGGTCAGGACGGAC & lmo2 sgRNA4 HRMA fwd \\
\hline TCACGCACACGCATGGTCA & lmo2 sgRNA4 HRMA rev \\
\hline AAACGCAGGTGTTCCGTAATGCCC & $\begin{array}{l}\text { notch1a knock-in sgRNA } \\
\text { cloning (antisense) }\end{array}$ \\
\hline TAGGGGGCATTACGGAACACCTGC & $\begin{array}{l}\text { notch1a knock-in sgRNA } \\
\text { cloning (sense) }\end{array}$ \\
\hline $\begin{array}{l}\text { CCCATCTGCCGGTGGCCAACGGCA } \\
\text { AGCCTATCCCAAACCCTCTGCTGG } \\
\text { GCCTGGACTCCACAAATGTGGGTG } \\
\text { GAGCTCAAGCA }\end{array}$ & $\begin{array}{l}\text { notch1a V5 HDR } \\
\text { template }\end{array}$ \\
\hline $\begin{array}{l}\text { TCATGAGCGCATGCACCACGATAT } \\
\text { C }\end{array}$ & notch1a V5-flanking fwd \\
\hline $\begin{array}{l}\text { TATGCCGAAGCTCCGCCTACTTGA } \\
\text { A }\end{array}$ & notch1a V5-flanking rev \\
\hline AAACGCTGCAGAGCCAGAGCCAGA & $\begin{array}{l}\text { notch3 knock-in sgRNA } \\
\text { cloning (antisense) }\end{array}$ \\
\hline TAGGTCTGGCTCTGGCTCTGCAGC & $\begin{array}{l}\text { notch3 knock-in sgRNA } \\
\text { cloning (sense) }\end{array}$ \\
\hline $\begin{array}{l}\text { AGCATGCTATTGCACCAGCAGGGC } \\
\text { AAGCCTATCCCAAACCCTCTGCTG } \\
\text { GGCCTGGACTCCACAGTGTTCCGT } \\
\text { AATGCCCAGCA }\end{array}$ & notch3 V5 HDR template \\
\hline $\begin{array}{l}\text { CTGACTCTAGACATGCAGAGTGCT } \\
\text { T }\end{array}$ & notch3 V5-flanking fwd \\
\hline $\begin{array}{l}\text { TTGGGCACATGCAGATAATGCTTA } \\
\text { G }\end{array}$ & notch3 V5-flanking rev \\
\hline TGTGTGTGTTTCCAGGTCCA & $\begin{array}{l}\text { npas4l bHLH HRMA fwd } \\
\text { (Marass et al., 2019) }\end{array}$ \\
\hline CGCAGGTTCCGTATCTCACT & $\begin{array}{l}\text { npas4l bHLH HRMA rev } \\
\text { (Marass et al., 2019) }\end{array}$ \\
\hline
\end{tabular}


Table 3.12: Oligonucleotides used in this thesis.

5' to 3' nucleotide sequence

AAACAAGGAGCATCTAAGGCGCGG

TAGGCCGCGCCTTAGATGCTCCTT

AAACACATCAGGAAGAGTGTGGAG

TAGGCTCCACACTCTTCCTGATGT

ATGAGTGTGTGTATCTGCATTGC

GCCAACGTGCCGATCC

CTCTGTTCTGCTGGTGATCTGC

\begin{tabular}{|c|c|}
\hline & PCR fwd \\
\hline TGTGTACCTCTCAGCTCCACACTC & $\begin{array}{l}\text { npas4l exon2 flanking } \\
\text { PCR rev }\end{array}$ \\
\hline $\begin{array}{l}\text { CGGGGGGTGTGTGTGCTGTCCAGC } \\
\text { TCTCTGAGGATGCTGTCGATGATG } \\
\text { CCCTCG }\end{array}$ & $\begin{array}{l}\text { npas4l Gal4 3' homology } \\
\text { arm, antisense }\end{array}$ \\
\hline $\begin{array}{l}\text { AAGCGAGGGCATCATCGACAGCAT } \\
\text { CCTCAGAGAGCTGGACAGCACACA } \\
\text { CACCCC }\end{array}$ & $\begin{array}{l}\text { npas4l Gal4 3' homology } \\
\text { arm, sense }\end{array}$ \\
\hline $\begin{array}{l}\text { ATCCGTCCAGCAGTGGCTCTGGTG } \\
\text { CCCAGCAGGAGGTGTGGTGAGAGT } \\
\text { GTGTGAAA }\end{array}$ & $\begin{array}{l}\text { npas4l Gal4 5' homology } \\
\text { arm, antisense }\end{array}$ \\
\hline $\begin{array}{l}\text { GCGGTTTCACACACTCTCACCACA } \\
\text { CCTCCTGCTGGGCACCAGAGCCAC } \\
\text { TGCTGGAC }\end{array}$ & $\begin{array}{l}\text { npas4l Gal4 5' homology } \\
\text { arm, sense }\end{array}$ \\
\hline TTGGTCCCTGCTGTGTTCTG & $\begin{array}{l}\text { npas4l Gal4 knock-in } \\
\text { detection fwd }\end{array}$ \\
\hline TCTCCACTGAAGCCAATCTATCTG & $\begin{array}{l}\text { npas4l Gal4 knock-in } \\
\text { detection rev }\end{array}$ \\
\hline AAACTCGTCCAGCAGTGGCTCTGG & $\begin{array}{l}\text { npas4l knock-in sgRNA } \\
\text { cloning (antisense) }\end{array}$ \\
\hline TAGGCCAGAGCCACTGCTGGACGA & $\begin{array}{l}\text { npas4l knock-in sgRNA } \\
\text { cloning (sense) }\end{array}$ \\
\hline
\end{tabular}

Primer name and description npas4l bHLH targeting sgRNA1 (antisense) (Marass et al., 2019) npas4l bHLH targeting sgRNA1 (sense) (Marass et al., 2019) npas4l bHLH targeting sgRNA2 (antisense) npas4l bHLH targeting sgRNA2 (sense) npas4l cDNA exon1 fwd1 npas4l cDNA exon1 fwd2 npas4l exon2 flanking npas4l exon2 flanking npas4l Gal4 3' homology 
Table 3.12: Oligonucleotides used in this thesis.

\begin{tabular}{|c|c|}
\hline $5^{\prime}$ to $3^{\prime}$ nucleotide sequence & $\begin{array}{l}\text { Primer name and } \\
\text { description }\end{array}$ \\
\hline CTACAGGATTGTGCTCTACAG & $\begin{array}{l}\text { npas4l qPCR fwd } \\
\text { (Reischauer et al., 2016) }\end{array}$ \\
\hline GTACGTTCTCAGACACATACAG & $\begin{array}{l}\text { npas4l qPCR rev } \\
\text { (Reischauer et al., 2016) }\end{array}$ \\
\hline CGCACGACACACACACTC & $\begin{array}{l}\text { npas4l sgRNA10 HRMA } \\
\text { fwd }\end{array}$ \\
\hline TGTGCTGTCCAGCTCTCTGA & $\begin{array}{l}\text { npas4l sgRNA10 HRMA } \\
\text { rev }\end{array}$ \\
\hline $\begin{array}{l}\text { AGGATGCTGTCGATGATGCCCTCG } \\
\text { TCTGTGGAGTCCAGGCCCAGCAGA } \\
\text { GGGTTTGGGATAGGCTTGCCAGAT } \\
\text { CTAGAAAGCCCTCCGCCACCTCCA } \\
\text { TCCAGCAGTGGCTCTGGTGCCCAG }\end{array}$ & npas 4 l V5 HDR template \\
\hline CTTGGTCCCTGCTGTGTTCT & npas4l V5-flanking fwd \\
\hline TGCATCAGTGTGTGTGCACTAGA & npas4l V5-flanking rev \\
\hline GGGGGCGGGATTTATTTCTGA & $\begin{array}{l}p d g f a b \text { promoterdeletion } \\
\text { PCR fwd1 }\end{array}$ \\
\hline TTCTCCGTGACGTAAGCCTG & $\begin{array}{l}p d g f a b \text { promoterdeletion } \\
\text { PCR rev1 }\end{array}$ \\
\hline AAACGTAGGAGATTGGTTAAGGAG & $\begin{array}{l}p d g f a b \text { sgRNA1 } \\
\text { (antisense) }\end{array}$ \\
\hline TAGGCTCCTTAACCAATCTCCTAC & $p d g f a b$ sgRNA1 (sense) \\
\hline AAACGGTGTACAGGATAAACCGTG & $\begin{array}{l}p d g f a b \text { sgRNA2 } \\
\text { (antisense) }\end{array}$ \\
\hline TAGGCACGGTTTATCCTGTACACC & $p d g f a b$ sgRNA2 (sense) \\
\hline AAACTCCCTCAGGAGTTCATCGAC & $\begin{array}{l}p d g f a b \text { sgRNA3 } \\
\text { (antisense) }\end{array}$ \\
\hline TAGGGTCGATGAACTCCTGAGGGA & $p d g f a b$ sgRNA3 (sense) \\
\hline AAACAGGTAAATTCTCCTCATTGT & $\begin{array}{l}\text { pdgfab sgRNA4 } \\
\text { (antisense) }\end{array}$ \\
\hline TAGGACAATGAGGAGAATTTACCT & pdgfab sgRNA4 (sense) \\
\hline AAACACTTTATGTTATGGCCTT & $\begin{array}{l}p d g f b a \text { sgRNA62 cloning } \\
\text { (antisense) }\end{array}$ \\
\hline TAGGAAGGCCATAACATAAAGT & $\begin{array}{l}p d g f b a \text { sgRNA62 cloning } \\
\text { (sense) }\end{array}$ \\
\hline
\end{tabular}


Table 3.12: Oligonucleotides used in this thesis.

\section{5' to $3^{\prime}$ nucleotide sequence}

\begin{tabular}{|c|c|}
\hline & \\
\hline ATTACAGCAGCCTGAACAGCG & $\begin{array}{l}p d g f b a \text { sgRNA62 HRMA } \\
\text { fwd }\end{array}$ \\
\hline CGTGCGATGTTTGATAGACGA & $\begin{array}{l}p d g f b a \text { sgRNA62 HRMA } \\
\text { rev }\end{array}$ \\
\hline AGCCATCATGACAATGACTCC & $p d g f b b$ HRMA fwd \\
\hline $\begin{array}{l}\text { TGAGAGAATAAAAGAGAAGTGAAC } \\
\text { TGA }\end{array}$ & $p d g f b b$ HRMA rev \\
\hline AAACGTGCTCACCCACCAAAGTCC & $\begin{array}{l}p d g f c \text { sgRNA46 cloning } \\
\text { (antisense) }\end{array}$ \\
\hline TAGGGGACTTTGGTGGGTGAGCAC & $\begin{array}{l}\text { pdgfc sgRNA46 cloning } \\
\text { (sense) }\end{array}$ \\
\hline CTCTGATCTCTGCTTTACCTCGTG & $\begin{array}{l}\text { pdgfc sgRNA46 HRMA } \\
\text { fwd }\end{array}$ \\
\hline CGCCTGCTGTTCTTACAACTG & $\begin{array}{l}p d g f c \text { sgRNA46 HRMA } \\
\text { rev }\end{array}$ \\
\hline AAACTCGCTGTCTGCTGGTGCA & $\begin{array}{l}p d g f d \text { sgRNA2 cloning } \\
\text { (antisense) }\end{array}$ \\
\hline TAGGTGCACCAGCAGACAGCGA & $\begin{array}{l}p d g f d \text { sgRNA2 cloning } \\
\text { (sense) }\end{array}$ \\
\hline GGTGAATTTACGAGAGGAGCTG & $p d g f d$ sgRNA2 HRMA fwd \\
\hline CCGCAGTTTCCACCACAGC & $p d g f d$ sgRNA2 HRMA rev \\
\hline TCTGGAGGACTGTAAGAGGTATGC & rpl13a qPCR fwd \\
\hline AGACGCACAATCTTGAGAGCAG & rpl13a qPCR rev \\
\hline $\begin{array}{l}\text { GCAGAATTCGCCACCATGATGGAA } \\
\text { AAACTGAAATCCGAGCA }\end{array}$ & tal1 pCS2z cloning fwd \\
\hline $\begin{array}{l}\text { TGCCTCGAGTCACCGCTGGGCATT } \\
\text { TCCG }\end{array}$ & tal1 pCS2z cloning rev \\
\hline GAGTTTCTTGTCAGGAGGGT & $\begin{array}{l}\text { tal1 qPCR fwd } \\
\text { (Reischauer et al., 2016) }\end{array}$ \\
\hline AAACGACGCAGGATCTCGTTCTTG & tal1 sgRNA11 (antisense) \\
\hline TAGGCAAGAACGAGATCCTGCGTC & tal1 sgRNA11 (sense) \\
\hline $\begin{array}{l}\text { CAAGAAACTCAGCAAGAACGAGAT } \\
\text { C }\end{array}$ & tal1 sgRNA11 HRMA fwd \\
\hline GTCCTGGTCGTTGAGGAGCT & tal1 sgRNA11 HRMA rev \\
\hline GAAATCAACGATGGTTCGCAG & $\begin{array}{l}\text { tal1 qPCR rev } \\
\text { (Reischauer et al., 2016) }\end{array}$ \\
\hline
\end{tabular}

Primer name and description $p d g f b a$ sgRNA62 HRMA fwd pdgfba sgRNA62 HRMA $p d g f b b$ HRMA fwd

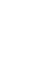


Table 3.12: Oligonucleotides used in this thesis.

\begin{tabular}{l|l}
$\mathbf{5}$ 'to 3 ' nucleotide sequence & $\begin{array}{l}\text { Primer name and } \\
\text { description }\end{array}$ \\
\hline CTCAGATCTGCAGGTGTTCTCC & $\begin{array}{l}\text { tmem121 sslp PCR fwd } \\
\text { (Reischauer et al., 2016) }\end{array}$ \\
\hline AGCGTTCCTCATATTCCTGATG & $\begin{array}{l}\text { tmem121 sslp PCR rev } \\
\text { (Reischauer et al., 2016) }\end{array}$ \\
\hline GCAGGATCCGCCACCATGGCTCCA & UAS:nls-mTagBFP2 \\
AAGAAGAAGCGTAAGGTAATGAGT & cloning fwd \\
AAAGGGGAGGAGTTAATTAAGGAG & \\
A & \\
\hline GCACTCGAGTTTAGTTCAGCTTGT & UAS:nls-mTagBFP2 \\
GTCCCAGCT & cloning rev \\
\hline GGCAAGCCTATCCCAAACCCTCTG & V5-F11 (Burg et al., 2016) \\
\hline GAGTCCAGGCCCAGCAGAG & V5-R3 (Burg et al., 2016)
\end{tabular}

\subsubsection{Plasmids}

Table 3.13: List of plasmids

\begin{tabular}{l|l} 
Plasmid & Citation/ cloning strategy \\
\hline DR274 & (Hwang et al., 2013) \\
\hline pCS2-nCas9n-nanos 3'UTR & (Moreno-Mateos et al., 2015) \\
\hline pCS2-nls-mTagBFP2 & cloned via BamHI/XhoI \\
\hline pCS2-npas4l & (Marass et al., 2019) \\
\hline pCS2z & (Horstick et al., 2015) \\
\hline pCS2z-etsrp & cloned via EcoRI/XhoI \\
\hline pCS2z-lmo2 & cloned via EcoRI/XhoI \\
\hline pCS2z-tal1 & cloned via EcoRI/XhoI \\
\hline pDB600 & (Balciunas et al., 2006) \\
Transposase) & \\
\hline pGTag-gal4-VP16-B-actin & (Wierson et al., 2020) \\
\hline pGTag-il11ra-sg7-p2a-Gal4-VP16 & cloned via BspQ1 and BfuAI \\
\hline pGTag-npas4l-sg10-p2a-Gal4- & cloned via BspQ1/BfuAI \\
VP16 & \\
\hline pT2-UASMCS-shortTol & (Gunawan et al., 2019) \\
\hline pT2-5xUAS:mTagBFP2 & cloned via BamHI/XhoI \\
\hline pT3TS-nCas9n & (Jao et al., 2013)
\end{tabular}




\subsubsection{Software}

Table 3.14: List of software

\begin{tabular}{l|l} 
Software & Source \\
\hline Anaconda & (Inc, 2020) \\
\hline BLAST & (Altschul et al., 1990) \\
\hline chopchop & (Labun et al., 2016) \\
\hline Google & Alphabet \\
\hline IGV 2.8.9 & (Thorvaldsdóttir et al., 2012) \\
\hline ImageJ (FIJI) & (Schindelin et al., 2012) \\
\hline Inkscape 0.92.5 & (Project, 2020) \\
\hline Mendeley Desktop 1.19.3 & Elsevier \\
\hline Office 365 & Microsoft \\
\hline Primerblast & (Ye et al., 2012) \\
\hline Python 3.7 & (Van Rossum and Drake Jr, 1995) \\
\hline R 4.0.3 "Bunny-Wunnies Freak & (R Core Team, 2020) \\
Out" & \\
\hline R Studio & (R Core Team, 2020) \\
\hline ShareLatex & (Henry Oswald, 2015) \\
\hline SMART & (Schultz et al., 1998; Letunic \\
\hline Snapgene 5.2.4 & et al., 2021) \\
\hline xMedia Recode & GSL Biotech \\
\hline Zen2.0 & (Dörfler, 2020) \\
\hline & ZEISS
\end{tabular}

\subsubsection{Databases}

Table 3.15: List of databases

\begin{tabular}{l|l|l} 
Database & URL & Citation \\
\hline Ensembl & www.ensembl.org & (Yates et al., 2019) \\
\hline InterPro & www.ebi.ac.uk/interpro/ & (Blum et al., 2020) \\
\hline PubMed & pubmed.ncbi.nih.gov & (Agarwala et al., 2018) \\
\hline RefSeq & www.ncbi.nlm.nih.gov/refseq/ & (O’Leary et al., 2015) \\
\hline Uniprot & www.uniprot.org & (Consortium, 2020) \\
\hline ZFIN & zfin.org & (Ruzicka et al., 2019)
\end{tabular}




\subsubsection{Services}

Sanger sequencing was performed by Microsynth Seqlab (Economy Run) and GATC (Eurofins Genomics). Synthetically produce crRNA and tracrRNA from Integrated DNA technologies were used for the knock-ins into npas 4 l (crRNA: CCAGAGCCACTGCTGGACGA), il11ra (crRNA: TAGGACTGAAGTATGTAATC). Also the universal guide RNA published by Wierson et al. (2020) to cut the knock-in vector in vivo was ordered from this company (crRNA: GGGAGGCGUUCGGGCCACAG). Codon-optimized mTagBFP2 was ordered as a GeneStrand from Eurofins Genomics. Oligonucleotides including primers and HDR templates for V5-tagging were ordered from Merck.

\subsection{Methods}

\subsubsection{Genome editing using CRISPR/Cas9 technology}

N20-NGG sequences in the region of interest were selected manually and checked for off-targets using the CRISPR design tool CHOPCHOP v2 (Labun et al., 2016) on the zebrafish Grcz10 genomic assembly. For knock-out generation, sgRNA templates were designed and cloned as described previously (Hwang et al., 2013; Gagnon et al., 2014) via BsaI and in vitro transcribed using the MEGAshortscript-T7 kit, followed by purification on an RNAcleanup column. The gRNA used to generate the Gal4 knock-in reporter was ordered as crRNA/tracrRNA duplex and assembled according to the user method provided by the Essner lab (https://sfvideo.blob.core.window s.net/sitefinity/docs/default-source/user-submitted-method/crispr-cas9-rnp -delivery-zebrafish-embryos-j-essnerc46b5a1532796e2eaa53ff00001c1b3c.pdf ?sfvrsn=52123407_10). The resulting sgRNA's or gRNA's were co-injected with Cas9 mRNA (300 pg) in a $2 \mathrm{nl}$ injection volume with $20 \%$ phenol red into zebrafish zygotes.

\subsubsection{1 npas4 $4 l^{\text {Pt(npas4l-V5)bns314 }}$}

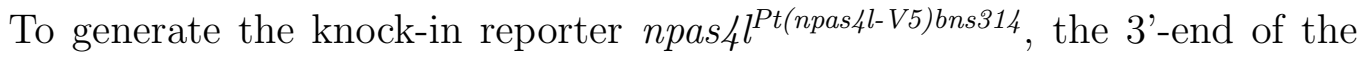
npas 4 l coding sequence was targeted using the gRNA target sequence 5'CCAGAGCCACTGCTGGACGA-3'. The HDR oligo donor was inserted after the codon for the endogenous Npas4l amino acid L625 and the coding sequence continued after the linker-V5 insertion. Knock-in efficiency was estimated by T7-endonuclease I assay for guide efficiency and flanking PCR on pools of injected embryos. 
The precise insertion was verified by Tag-specific and flanking PCR resolving both the 3' and 5' insertion sites.

\subsubsection{2 npas $4 l^{\text {Pt(npas4l-p2A-Gal4-VP16)bns313 }}$}

To generate the knock-in reporter npas $4 l^{\text {Pt(npas4l-p2A-Gal4-VP16)bns313, hence- }}$ forth named npas $4 l^{+}$reporter, the 3'-end of the npas $4 l$ coding sequence was targeted using the gRNA target sequence 5'-CCAGAGCCACTGCTGGAC GA-3'. The transgenic donor was inserted after the codon for the endogenous Npas4l amino acid L625, thus truncating the Npas4l peptide sequence by 22 amino acids. The pGTag-p2A-Gal4/VP16 donor was cloned as described in (Wierson et al., 2020) using oligos to assemble the 5' and 3'-homology arms and cloning them into the pGTag-Gal4-VP16 via BfuAI and BspQI. Efficiency of the guide was tested by high resolution melt analysis (HRMA). Knock-in efficiency was estimated by injections into a $U A S$ :GFP background followed by screening for GFP-mosaicism.

Founders were screened by outcrossing the injected individuals and screening for GFP-fluorescence in at least 300 embryos. Precise 5'-insertion of the cassette F1-progeny of positive transmitters was confirmed by PCR on the gDNA and cDNA level. PCR for the vector backbone of the pGTag donor plasmid in the bns313Pt allele indicated vector concatemer insertions; the genomic 3' insertion sequence has not been resolved by PCR.

\subsubsection{3 npas4 $l^{P t\left(+36 b p-n p a s 4 l-p 2 A-G a l_{4}-V P 16\right) b n s 423}$}

$n p a s 4 l^{P t\left(+36 b p-n p a s 4 l-p 2 A-G a l_{4}-V P 16\right) b n s 423}$, a mutant version of the $n p a s 4 l^{+}$reporter (henceforth named npas $4 t^{-}$reporter), was generated by inducing an in-frame indel in the domain encoding the DNA-binding bHLH-domain to retain the reading frame and reporter expression but abolish Npas4l transcriptional activity. I aimed to delete the entire bHLH domain encoding region using one previously published sgRNA target sequence (Marass et al., 2019), combined with a newly designed sgRNA. Injected fish and potential founders were screened by PCR. I recovered a +36 bp insertion at the end of exon2 that showed a strong phenotype and correct splicing as determined by PCR spanning the coding sequence from exon1 to the Gal4-insertion. I used this allele as npas4t reporter bns423.

\subsubsection{4 tal1 $^{\text {bns } 497}$ and tal1 $^{\text {bns } 498}$}

For the generation of tal1 mutant alleles, I targeted the region encoding the DNA-binding bHLH domain in the 4th exon using the sgRNA target 
sequence 5'-CAAGAACGAGATCCTGCGTC-3'. Injected fish and potential founders were screened by HRMA. I recovered the $-6 \mathrm{bp}$ in-frame deletion allele bns498 leading to the loss of R228 and L229 in the second helix of the HLH-motif and the -7 bp out-of-frame deletion allele bns497 leading to a premature termination codon after R228 that is predicted to truncate the peptide sequence by 97 amino acids.

\subsubsection{5 etsrp ${ }^{\text {bns422 }}$ and etsrp ${ }^{\text {bns426 }}$}

For the generation of etsrp mutant alleles, I targeted the region encoding the DNA-binding ETS domain in the 6th exon using the sgRNA target sequence 5'-AAGTTGGACTGGTGATGGCT-3'. Injected fish and potential founders were screened by HRMA. I recovered the $-9 \mathrm{bp}$ in-frame deletion allele bns426 leading to the loss of D265 G266 and W267 and the -4 bp out-offrame deletion allele bns422 after G264 leading to an altered peptide sequence AGSLKCQIPLRWRSGGASVKTSLK* followed by a premature termination codon at position 289 , that is predicted to truncate the peptide sequence by 78 amino acids.

\subsubsection{6 lmo $\mathscr{2}^{b n s 499}$ and $\operatorname{lmo} \mathscr{2}^{b n s 500}$}

For the generation of lmo2 mutants, I targeted the region encoding the beginning of the second LIM domain in the 3rd exon using the sgRNA target sequence 5'-TTCCTGTGAAAAGAGGATCC-3' with the aim to split the two conserved LIM domains of this scaffolding protein. Injected fish and potential founders were screened by HRMA. I recovered the $-6 \mathrm{bp}$ in-frame deletion allele bns500 leading to the replacement of I101 R102 A103 with T101 and the +2 bp out-of-frame indel allele bns499 after R100 leading to an altered peptide sequence TTGPLK* followed by a premature termination codon at position 107, that is predicted to truncate the peptide sequence by 52 amino acids.

\subsubsection{7 il11ra ${ }^{P t\left(i l 11 r a-p 2 a-G a l_{4}-V P 16\right) b n s 424}$}

To generate the knock-in reporter il11ra ${ }^{P t(i l 11 r a-p 2 a-G a l 4-V P 16) b n s 424}$, exon 9 of the il11ra genomic sequence was targeted using the gRNA target sequence 5'-TAGGACTGAAGTATGTAATC-3'. The transgenic donor was inserted after the codon for the endogenous Il11ra amino acid D327, thus truncating the Npas4l peptide sequence by 74 amino acids. The pGTag-p2A-Gal4/VP16 donor was cloned as described in (Wierson et al., 2020) using oligos to assemble the 5' and 3'-homology arms and cloning them into the pGTag-G 
via BfuAI and BspQI. Efficiency of the guide was tested by high resolution melt analysis (HRMA). Knock-in efficiency was estimated by injections into the $\mathrm{Tg}(U A S: G F P)^{\text {nkuasgfp1a }}$ background followed by screening for GFPmosaicism.

Founders were screened by outcrossing the injected individuals and screening for GFP-fluorescence in at least 300 embryos. Precise 5'-insertion of the cassette F1-progeny of positive transmitters was confirmed by PCR on the gDNA level.The genomic 3' insertion sequence has not been tested by PCR yet.

\subsubsection{8 notch1 $a^{\text {Pt(notch } 1 a-V 5) N N-107}$}

To generate the knock-in reporter notch $1 a^{P t(n o t c h 1 a-V 5) N N-107}$, a sequence motif with relatively low evolutionary conservation in the last exon (Burg et al., 2016) of notch1a was targeted using the gRNA target sequence 5'-TCTGG CTCTGGCTCTGCAGC-3'. The HDR oligo donor was inserted after the codon for the endogenous Notch1a amino acid G2230 and the coding sequence continued after the linker-V5 insertion. Knock-in efficiency was estimated by T7-endonuclease I assay for guide efficiency and flanking PCR on pools of injected embryos.

The precise insertion was verified by Tag-specific and flanking PCR resolving both the 3' and 5' insertion sites. The V5 signal was detectable but too low for the intended purpose.

\subsubsection{9 notch3 $3^{\text {Pt(notch3-V5) }}$}

To generate the knock-in reporter notch $3^{P t(n o t c h 3-V 5)}$, a sequence motif with relatively low evolutionary conservation in the last exon (Burg et al., 2016) of notch3 was targeted using the gRNA target sequence 5'-GGGCATTAC GGAACACCTGC-3'. The HDR oligo donor was inserted after the codon for the endogenous Notch3 amino acid Q2275 and the coding sequence continued after the linker-V5 insertion. Knock-in efficiency was estimated by T7-endonuclease I assay for guide efficiency and flanking PCR on pools of injected embryos.

The precise insertion was verified by Tag-specific and flanking PCR resolving both the 3' and 5' insertion sites. The V5 signal was detectable but too low for the intended purpose. 


\subsubsection{0 pdgfab ${ }^{\text {bns322 }}$ (promoter deletion)}

For the generation of a $p d g f a b$ promoter deletion allele, I targeted the putative promoter region of $p d g f a b$ using a mix of four different guide RNA's. Injected fish and potential founders were screened by PCR. I recovered a $-3260 \mathrm{bp}$ deletion allele bns322 removing parts of the promoter region as well as the transcriptional start site and the first two exons. The mutant allele did not cause a detectable phenotype in homozygous fish and has therefore not been analyzed in detail.

\subsubsection{1 pdgfba}

For the generation of a pdgfba mutant allele, I targeted the region encoding the conserved PDGF/VEGF homology domain in the 4th exon using the sgRNA target sequence 5'-TGAAGGCCATAACATAAAGT-3'. Injected fish and potential founders were screened by HRMA. I recovered the $-10 \mathrm{bp}$ out-of-frame deletion allele bns323 leading to a premature termination codon after P115 that is predicted to truncate the peptide sequence by 348 amino acids. Preliminary data suggests that this mutant shows a reduction in hindbrain pericytes and is going to be analyzed by Koji Ando (collaborative project, manuscript in preparation).

\subsubsection{2 pdgfbb $b^{\text {bns324 }}$}

For the generation of a $p d g f b b$ mutant allele, I used founder crosses injected previously by Claudia Gerri (unpublished results). I recovered the $-26 \mathrm{bp}$ out-of-frame deletion allele bns324 leading to a premature termination codon after S66 that is predicted to truncate the peptide sequence by 416 amino acids. The mutant allele did not cause a detectable phenotype in homozygous fish and has therefore not been analyzed in detail.

\subsubsection{3 pdgfc ${ }^{\text {bns325 }}$}

For the generation of a $p d g f c$ mutant allele, I targeted the region encoding the conserved PDGF/VEGF homology domain in the 5th exon using the sgRNA target sequence 5'-GGACTTTGGTGGGTGAGCAC-3'. Injected fish and potential founders were screened by HRMA. I recovered the $-5 \mathrm{bp}$ out-offrame deletion allele bns325 leading to a premature termination codon after C295 that is predicted to truncate the peptide sequence by 48 amino acids. The mutant allele did not cause a detectable phenotype in homozygous fish and has therefore not been analyzed in detail. 


\subsubsection{4 pdgfd $d^{\text {bns326 }}$}

For the generation of a $p d g f d$ mutant allele, I targeted the region encoding the conserved PDGF/VEGF homology domain in the 6th exon using the sgRNA target sequence 5'-GCTGCACCAGCAGACAGCGA-3'. Injected fish and potential founders were screened by HRMA. I recovered the -11 bp out-offrame deletion allele bns326 leading to a premature termination codon after P303 that is predicted to truncate the peptide sequence by 77 amino acids. The mutant allele did not cause a detectable phenotype in homozygous fish and has therefore not been analyzed in detail.

\subsection{2 npas4l-alleles and genotypes of the crosses used to generate the data}

For technical reasons, I used several different npas4l mutant and reporter alleles throughout this study. The detailed genotypes are listed in Table 3.16. The reasons for these choices are outlines in the section below.

Table 3.16: npas4l genotypes of the animals by Figures.

\begin{tabular}{l|l} 
Figure & npas4l alleles \\
\hline Figure 4.3-4.4 & bns314/bns314 \\
\hline Figure 4.6- 4.15 & bns313/+ \\
\hline Figure 4.16 & bns313/+ (injected) \\
\hline Figure 4.18 & bns423/+ (left) and bns423/bns297 (right) \\
\hline Figure 4.19A & bns313/bns313 (left) and bns423/bns423 (right) \\
\hline Figure 4.19B & bns423/+ (left) and bns423/bns423 (right) \\
\hline Figure 4.20-4.21 & bns423/bns297 \\
\hline Figure 4.22-4.27 & bns423/+ (+/-) and bns423/bns297 (-/-) \\
\hline Figure 4.29 & bns313/+ \\
\hline Figure 4.31-4.34 & bns313/+ \\
\hline Figure 4.35 & bns423/bns423 \\
\hline Figure 4.36 & bns423/+ (+/-) and bns423/bns297 (-/-) \\
\hline Figure 4.37-4.38 & bns313/+
\end{tabular}

As a benchmark for strong npas4l mutant alleles, I used the m39 chromosomal aberration allele, in which the npas $4 l$ gene is missing and therefore a known null allele. However, genotyping of this allele is difficult and several other genes alongside npas $4 l$ are deleted in it. Similarly, in-crosses of the bns423 allele were problematic as different levels of Gal4 or GFP may contribute to variability in observed phenotypes. Therefore, I used intercrosses of the bns423 allele to the crispr allele bns297 for several figures, 
as this causes a strong phenotype indistinguishable from homozygous m39 allele mutants, but can be genotyped by HRMA as described in Marass et al. (2019).

In addition to that, homozygous npas $4 l$ mutants show strong phenotypes and mutations in this gene may theoretically cause minor heterozygous defects as well. Although no such defects of cloche/npas 4 l have been reported so far and I did not notice anything like that in this study, I was aware of the potential issue and tried to avoid doing experiments with downstream mutants in a npas4l heterozygous mutant background. Therefore, I used the bns313 reporter allele outcrosses in the tal1, etsrp and lmo2 mutant background to generate all data for these mutants.

\subsubsection{Zebrafish methods}

\subsubsection{Zebrafish maintenance}

Embryos and larvae were raised under standard conditions (Westerfield, 1993) and staged as described previously (Kimmel et al., 1995).

All procedures performed on animals conform with the guidelines from Directive 2010/63/EU of the European Parliament on the protection of animals used for scientific purposes and were approved by the Animal Protection Committee (Tierschutzkommission) of the Regierungspräsidium Darmstadt (references: B2/1041 and B2/1218).

\subsubsection{Zebrafish breeding}

Zebrafish matings were set up in mating tanks during late afternoon or evening to reduce the amount of time the fish spend outside the system and thereby decrease potential stress. Males and females were separated by a plastic divider.

Between 8 and $11 \mathrm{AM}$ in the morning, the dividers were pulled to allow mating and spawning of eggs. Before embryos were collected from breeding tanks, the adults were put back to reduce stress to the animals. Eggs were then collected by sieving the water through tea sieves and rinsed into petri dishes using egg water. 


\subsubsection{Handling of zebrafish embryos}

The embryos were then cleaned from unfertilized embryos under a widefield stereomicroscope at $\geq 3 \mathrm{hpf}$ and around $24 \mathrm{hpf}$. Embryos were spread in the dish with a maximum density of 80 embryos in a $9 \mathrm{~cm}$ petri dish with $30-50 \mathrm{ml}$ eggwater. For stage-sensitive and early imaging experiments, this density was reduced to $<50$ embryos per dish.

Embryos that were intended to be raised into adulthood were bleached between tailbud stage and $28 \mathrm{hpf}$ by collecting the embryos in a sieve and exposing them two times to $0.003 \% \mathrm{NaOCl}$ for $5 \mathrm{~min}$ each followed by $5 \mathrm{~min}$ rinsing in egg water. After that, embryos were rinsed in eggwater again and collected in a clean petri dish. $60 \mu \mathrm{l}$ pronase $(30 \mathrm{mg} / \mathrm{ml})$ was added to dechorionated the embryos overnight. On the next day, the pronase and chorions were washed out. Free-swimming larvae were brought to the system as soon as they developed a swim bladder (4-5 dpf).

Embryos that were treated or imaged before natural hatching were dechorionated using either manual dechorionation by forceps or enzymatic dechorionation by adding $200-1000 \mu \mathrm{l}$ pronase $(30 \mathrm{mg} / \mathrm{ml})$ per dish for $20 \mathrm{~min}$ (high concentration) to 3 hours (lower concentration). Early embryos (before $48 \mathrm{hpf}$ ) were dechorionated in agarose-coated dishes to prevent them from sticking to the plastic dish.

Embryos and larvae that needed to be imaged after $24 \mathrm{hpf}$ were treated with $0.003 \%$ phenylthiourea (PTU) between 22 and $26 \mathrm{hpf}$ to avoid pigmentation.

\subsubsection{Microinjections}

One day before the injection needles were pulled. Injection plates were prepared placing a plastic mold (institute workshop) in a petridish filled with heated $2 \%$ agarose in eggwater. After cooling and removing the mold, these plates can be stored for several weeks at $+4^{\circ} \mathrm{C}$. Matings of the desired backgrounds were set up with dividers for the injection next day.

On the day of injection, injections mixes were prepared by adjusting them to the right concentration and adding $20 \%$ phenolred to the mixture to indicate the $\mathrm{pH}$ of the solution and monitor injection success. Only mixtures colored in pale red were injected. 
For the injections, dividers were pulled and the embryos collected 3-5 min after spawning. Needles were opened using forceps and loaded using $20 \mathrm{\mu l}$ microloader tips. The loaded needle was positioned in the micro manipulator and the desired injection volume adjusted by injecting droplets into a drop of mineral oil placed on a microscale slide.

Eggs were loaded into the rows of the injection plate. For yolk injections, 2-3 nl of injection mix were injected into the middle of the yolk. For cellular injections, embryos were turned using the tip of the needle and $2 \mathrm{nl}$ injected into the zygote.

After injection, embryos were rinsed into petri dishes using egg water and cleaned at around 3, 6 and $24 \mathrm{hpf}$ to remove dead and deformed embryos.

\subsubsection{Zebrafish screening}

Several knock-in, knock-out and transgenic lines were generated in the course of this thesis project. A general screening procedure helped to standardize the process of screening for injection success, founders and transmitters at the various stages.

In the P0 generation, fish that were supposed to show fluorescence upon injection were screened beginning at $24 \mathrm{hpf}$ twice a day to observe the potential expression pattern. Embryos showing at least one positive cell were raised.

In addition to screening for fluorescence whenever possible, molecular biology techniques on gDNA were used to analyze the injection quality in a qualitative and quantitative manner in injected $\mathrm{P} 0$ embryos and larvae. For the assessment of CRISPR/Cas9 cleavage efficiency, standard HRMA was used as a qualitative and T7-endonuclease assay as a quantitative measure on single embryos. In simple cases like the generation of knock-out mutants, only HRMA was used due to its simplicity. The knock-in of non-fluorescent donor constructs was confirmed by PCR with one primer placed in the donor construct sequence and the other in the genomic sequence flanking the insertion site.

For founder screening, outcrosses were preferred for the generation of knock-out and standard transgenic lines and in-crosses for the generation of 
knock-in lines. Although in-crosses have a greater risk of accumulating offtarget mutations in the genetic background and were therefore never raised to establish the allele, in-crosses doubled the number of fish per mating that could be screened simultaneously and were therefore preferred for a prescreening when the probability of finding a founder was expected to be equal to or lower than one in five (and subsequently the probability of finding a founder transmitting two alleles less than 4\%). In such cases however, the two potential founders of the incross were outcrossed again separately to generate the line.

In addition to the methods described above, a pre-screening for npas $4 l-V 5$ founders by PCR on sperm samples was used to screen fast through a large number of fish (Lebcir et al., 2016). For that, fish were not fed one day before the treatment and males were separated from females. The adult males were anaesthetized with $0.01 \%$ Tricaine solution and turned on the dorsal side. Two fingers were gently placed on the lateral sides of the fish and the sperm collected in a glass capillary. The DNA was lysed using $50 \mathrm{mM} \mathrm{NaOH}$ as described in the section for gDNA isolation and used in PCR reactions in a 1:10-1:100 dilution.

For fluorescent lines, the F1 embryos were pre-sorted based on reporter expression. However, all F1 adults were later genotyped and analyzed by molecular biological methods. For lines that were screened by fluorescence before, transmitters were tested for precision of the insertion by PCR amplification and sequencing of the insertion site. Also, founders were tested for potential vector artifacts. Knock-in lines for non-fluorescent insertions were tested by PCR for presence of the vector and further tested by PCR spanning the entire locus to test for precise insertions. F1 adults for knock-out alleles were tested by HRMA for the presence of indels. PCR products of potential transmitters were cloned into pGEM-T Easy vectors according to manufacturers instructions and sequenced using M13 forward primers.

All F2-generations were raised from a single identified (sequenced) F1transmitter by outcrossing it to wild-type fish.

\subsubsection{Transplantation}

Transplantation plates with pockets for single embryos (1.5\% agarose in E2medium) and petri dishes with agarose coating were prepared in advance. 
$20 \mathrm{ml} 1.5 \mathrm{mg} / \mathrm{ml}$ pronase solution and $500 \mathrm{ml} 200 \mu \mathrm{g} / \mathrm{ml}$ Streptomycin in eggwater were prepared as well. Embryos were only handled with glass pipettes to avoid their destruction.

Embryos in most advanced stages were dechorionated first to delay their progression and synchronize the donors and hosts. After about 10 min treatment in the $20 \mathrm{ml}$ pronase solution, embryos were decanted and the solution re-used for the next batch of embryos. Embryos were then rinsed immediately and gently decanted into an agarose coated dish. After about $10 \mathrm{~min}$, dirt, egg shells and damaged embryos were removed. Slight agitation may help in the dechorionation of remaining embryos.

Pockets in water-filled transplantation plates were then filled with dechorionated embryos. Importantly, during the transfer of embryos no embryo may touch the liquid surface as surface tension destroys it. Donor cells were then taken from the inner cell mass and transferred into the target tissue in the host embryos at the oblong to dome stage. After the cell transplantations, embryos were kept in agarose coated dishes with water and Streptomycin and checked regularly for dead embryos.

\subsubsection{Anesthesia of adult zebrafish}

Adult zebrafish were anaesthetized by exposure to $0.01 \%$ tricaine in egg water. The gill movement was monitored, and the fish taken out of the anesthesia when gill movements slowed to less than one beat per second. Time outside the water was reduced to a minimum to reduce stress and harm to the animals.

After procedures were finished, fish were allowed to recover by transferring them into a tank with fresh water and by keeping them single for a few minutes to ensure full recovery before mixing them with other fish again.

\subsubsection{Zebrafish genotyping}

For genotyping before $5 \mathrm{dpf}$, the anaesthetized embryos/larvae were transferred into test tubes and killed by exposing them to $95^{\circ} \mathrm{C}$ for 5 seconds. For the genotyping of adults, a tailfin tip biopsy of anaesthetized adults was cut using a scalpel or sterilized fine scissors. The biological material was then used to extract gDNA for genotyping. 


\subsubsection{Zebrafish killing}

Zebrafish were killed in $0.01 \%$ tricaine in egg- or system water. A maximum of 5 fish per liter were killed at the same time. After transfer to the killing tank for $30 \mathrm{~min}$, death was ensured by decapitation or freezing of the cadavers at $-20^{\circ} \mathrm{C}$. Cadavers were stored in freezer chests up to cremation.

\subsubsection{Molecular biology}

\subsubsection{1 gDNA-extraction}

For fast DNA extraction, $100 \mu \mathrm{l} 50 \mathrm{mM} \mathrm{NaOH}$ solution were added to the biological material. The suspension was incubated for $10 \mathrm{~min}$ at $95^{\circ} \mathrm{C}$ to lyse the tissue chemically. $10 \mu \mathrm{l} 1 \mathrm{M}$ Tris- $\mathrm{HCl}$ buffer $(\mathrm{pH} 8)$ were added and mixed to buffer the solution. The gDNA collected by this method can be used directly, frozen or stored for short times at $+4^{\circ} \mathrm{C}$.

For long-term storage and downstream processes that required higher quality gDNA, $3 \mu \mathrm{g} / \mathrm{ml}$ proteinase $\mathrm{K}$ dissolved in $50 \mu \mathrm{l} 10 \mathrm{mM}$ Tris-Hcl pH 7.4 solution was added to the genetic material. The mixture was incubated for 3 hours or overnight at $55^{\circ} \mathrm{C}$ to lyse the tissue enzymatically. The proteinase $\mathrm{K}$ was then inactivated by $10 \mathrm{~min}$ incubation at $95^{\circ} \mathrm{C}$. The gDNA collected by this method generally worked better in challenging applications and could be stored frozen or at $+4^{\circ} \mathrm{C}$ for a longer time compared to the $\mathrm{NaOH}$ lysis protocol.

To purify old or degraded gDNA samples as well as to concentrate them, a standard precipitation was used independent of the initial lysis method. One volume of isopropanol was mixed into the gDNA solution and mixed by inversion of the tube or pipetting. The solution was precipitated by centrifugation at maximum speed for $10 \mathrm{~min}$ at room temperature. The supernatant was then discarded, replaced by $200 \mu \mathrm{l} 70 \%$ ethanol and centrifuged again for $5 \mathrm{~min}$ at room temperature. After careful removal of the supernatant, the remaining DNA pellet was dried at room temperature for about 15-20 min. The DNA was then eluted in 5-30 $\mu$ l elution buffer or water and used directly or stored for later use at $+4^{\circ} \mathrm{C}$.

\subsubsection{RNA-extraction}

Biological material was placed in a $1.5 \mathrm{ml}$ reaction tube with $200 \mu \mathrm{l}$ Trizol reagent and three metal bullets. The mixture was homogenized in the bullet 
blender for $3 \mathrm{~min}$ at room temperature and the supernatant transferred into a new tube.

\subsubsection{DNA/RNA quantification and quality assessment}

To measure the quality of purified DNA and RNA, samples were analyzed by Nanodrop photometric spectroscopy and/or agarose gel electrophoresis. For photometric analysis the optical density of the sample at different wavelengths was recorded compared to a solvent control. Concentrations were estimated by relative absorption at $260 \mathrm{~nm}$ whereas the ratio between $260 / 280 \mathrm{~nm}$ absorption served as a proxy for purity and protein concentration. A ratio between 1.7 and 2.1 was considered to indicate reasonable quality whereas a lower ratio indicated potential protein contaminations. High peaks at $220 \mathrm{~nm}$ are indicators of high salt concentrations or remaining solvents in the sample that may interfere with downstream applications.

Since free nucleotides that indicate impure or degraded samples show a clean $260 \mathrm{~nm}$ absorption peak as well, a nanodrop measurement might miss some important indicators of poor quality. For this reason, agarose gel electrophoresis was used to determine indicators of RNA/DNA quality. The following list is an overview over some of the problems that were regularly checked by this method:

- gDNA remains in the upper pocket and does not migrate into the gel. Sheared and degraded gDNA migrates into the gel and shows as a smear.

- Isolated eukaryotic RNA should contain a mixture of bands, but also show sharp 28S and 18S rRNA bands. An intensity ratio of 2:1 between these crisp two bands can serve as an indicator of good RNA quality, whereas a smear of RNA indicates degradation of the RNA in the sample.

- Synthetic mRNA should form a crisp band at the expected size. Bands of a larger size indicate incomplete digestion of the template DNA, whereas a smear below the expected band is a sign of degradation.

- Undigested plasmid DNA should be visible as two bands, one running above the expected size (relaxed) and one running below it (supercoiled). After digest, only bands of the expected sizes should appear whereas the pattern described above indicates incomplete digestion. 
- Smears of plasmid DNA after digest with restriction enzymes or T7endonucleases are indicators of unspecific activity that can be caused through excess amounts of enzymes, prolonged incubation, wrong buffers and other problems.

- Strong low molecular weight bands of less than 200 basepairs in PCR samples often indicate primer dimers that can reduce the efficiency of the PCR reaction.

- Bands of the wrong size or numerous bands in a PCR sample may indicate unspecific amplification caused by numerous problems such as unspecific binding of the primers to the template.

In addition to that, agarose gel electrophoresis allows the estimation of DNA/RNA concentration in samples by comparing a given sample with different dilutions of a DNA/RNA standard of similar length and known concentration.

\subsubsection{Agarose gel electrophoresis}

Agarose gels were prepared in a concentration range between 3\% $(<200 \mathrm{bp})$ and $1 \%(>3000 \mathrm{bp})$ in $1 \mathrm{xTBE}$ buffer $(\mathrm{w} / \mathrm{v})$. The agarose was solved through microwaving and cooled to about $60^{\circ} \mathrm{C}$ before adding and mixing in $5 \mathrm{\mu l}$ SYBR Safe to visualize DNA bands. Gels were run with constant voltage of 140-160 V for 40-70 min depending on gel size and required resolution.

\subsubsection{5 cDNA first strand synthesis}

First strand cDNA was synthesized using the Maxima cDNA synthesis kit with dsDNAse according to manufacturer instructions. $500 \mathrm{ng}$ total RNA were used and cleaned from traces of residual gDNA in a $10 \mu l$ DNAse treatment reaction for 2 Minutes at $37^{\circ} \mathrm{C}$. The cDNA reactions were then set up in a $20 \mu \mathrm{l}$ scale and incubated sequentially for $10 \mathrm{~min}$ at $25^{\circ} \mathrm{C}, 15 \mathrm{~min}$ at $50^{\circ} \mathrm{C}$ and the reaction terminated for 5 min at $85^{\circ} \mathrm{C}$. cDNA was stored at $-20^{\circ} \mathrm{C}$ or used directly.

\subsubsection{6 in vitro transcription}

in vitro transcribed mRNA was synthesized using the the SP6 mMessage mMachine kit in a 10-20 $\mathrm{\mu l}$ volume. The reaction was assembled according to kit instructions and incubated for $2 \mathrm{~h}$ at $37^{\circ} \mathrm{C}$ for a maximum yield. After DNAse digest for $15 \mathrm{~min}$, the mRNA was cleaned using the RNA clean and concentrator kit. Depending on the expected yield, the RNA was dissolved 
in $12-22 \mu \mathrm{l} \mathrm{H}_{2} \mathrm{O}$. $1 \mu \mathrm{l}$ aliquots were frozen down at $-80^{\circ} \mathrm{C}$ and $2 \mathrm{x} 1 \mu \mathrm{l}$ kept for analyses on both the Nanodrop photometer and gel. RNA was never diluted before freezing, but instead diluted after thawing.

\subsubsection{Primer design}

Primers were designed by hand with a GC content of $40-60 \%$ and a melting temperature of ideally $58-62^{\circ} \mathrm{C}$. PrimerBLAST (https://www.ncbi.nlm.nih.g ov/tools/primer-blast/) was used to optimize primers and check for off-target binding sites.

\subsubsection{Polymerase chain reaction (PCR)}

Primers were designed as described in section 3.2.4.7 and used in a $500 \mathrm{nM}$ concentration. The PCR program was chosen according to the specific attributes of the polymerase and primers (compare to section 3.1.2.8). For cloning, proof reading polymerases were used whereas KAPA2G was used for other tests such as colony PCR and detection of modifications.

PCR programs included initial denaturation, 30-37 cycles of denaturation, annealing and extension followed by a final extension step and storage at room temperature.

In general, a denaturation at $95^{\circ} \mathrm{C}$ (PrimeSTAR) or $98^{\circ} \mathrm{C}$ (KAPA2G or Phusion) was chosen. Both timing and temperature were copied from the manufacturers recommendations and not modified.

Annealing temperatures were not only chosen according to the primer but also to the polymerase and calculated according to the Thermo Fisher Scientific Tm-calculator tool (https://www.thermofisher.com/de/de/home/brand $\mathrm{s} /$ thermo-scientific/molecular-biology/molecular-biology-learning-center $/ \mathrm{m}$ olecular-biology-resource-library/thermo-scientific-web-tools/tm-calculator. html). For PrimeSTAR max, the Tm was kept constant at $55^{\circ} \mathrm{C}$ according to manufacturer instructions. For PrimeSTAR, an annealing time of 5 seconds and for other polymerases an annealing time of 15 seconds were used.

All Extensions were performed at $72^{\circ} \mathrm{C}$. For KAPA2G, only 1 s elongation time was used for templates $<1 \mathrm{~kb}$ length. Apart from that manufacturer recommendations for the elongation times were modified by adding 10-30s as a safety margin. Final extensions were set to the maunfacturer recommendations and not changed throughout the study. 


\subsubsection{High resolution melt analysis (HRMA)}

For HRMA, $500 \mathrm{~nm}$ primer, $1 \mu \mathrm{l}$ gDNA template and $5 \mu \mathrm{l}$ Dynamo Color Flash SyBR Green qPCR master mix were used in a $10 \mu l$ reaction. A qPCR reaction was performed, the PCR products melted at $95^{\circ} \mathrm{C}$, re-annealed and slowly melted again. The melting point of the double strands was recorded and the resulting fluorescence profile used to determine mismatches and other modification present in the sample.

\subsubsection{T7-endonuclease assay}

A 300-1000 bp area surrounding the editing site in injected and uninjected embryos was amplified by PCR and cleaned up using the GeneJET PCR purification kit. $200 \mathrm{ng}$ of the purified PCR product was used in a $19 \mu \mathrm{l}$ volume supplemented with NEB buffer 2 (supplied with the enzyme). The mixtures were boiled for $5 \mathrm{~min}$ at $95^{\circ} \mathrm{C}$ before slowly being cooled in a thermocycler with $-2^{\circ} \mathrm{C} / \mathrm{s}$ from $95-85^{\circ} \mathrm{C}$ and with $0.1^{\circ} \mathrm{C} / \mathrm{s}$ from $85-25^{\circ} \mathrm{C}$.

$1 \mu \mathrm{l}$ T7 Endonuclease I was then added to the solution and incubated for exactly $15 \mathrm{~min}$ at $37^{\circ} \mathrm{C}$. The reaction was stopped by adding $1.5 \mu \mathrm{l} 25 \mathrm{mM}$ EDTA and the products analyzed by agarose gel electrophoresis.

\subsubsection{Quantitative real-time PCR (qPCR)}

Total RNA was isolated from pools of 10 embryos at the tailbud stage using Trizol and purified by 2-propanol precipitation. First strand synthesis was performed using the Maxima First Strand cDNA Synthesis kit with $1 \mu \mathrm{g}$ RNA template as described in section 3.2.4.5. $0.5 \mu \mathrm{l}$ of the resulting first strand cDNA was used in a $10 \mu \mathrm{l}$ RT-qPCR reaction. qPCR was performed in technical duplicates using a CFX Connect Real-Time PCR system and fold changes calculated using the $2-\Delta \Delta \mathrm{Ct}$ method.

\subsubsection{Cloning of constructs using restriction enzymes}

All constructs were cloned using conventional restriction enzymes. About 3-5 $\mathrm{\mu g}$ plasmid DNA were digested as starting material. The insert was amplified by PCR using the primers indicated in section 3.1.2.8 and digested using the enzymes indicated in the plasmid description (compare to section 3.1.2.9). 30 fmol plasmid and $120 \mathrm{fmol}$ insert were mixed with T4-DNA ligase and incubated for 15 min- 1 hour before half of the mix was transformed into E. coli while the rest was ligated further over night at $4^{\circ} \mathrm{C}$. Religated vector 
without insert served as negative control. The constructs were then transformed into competent E.coli by $45 \mathrm{~s}$ heat shock followed by $2 \mathrm{~min}$ on ice and regeneration for $1 \mathrm{~h}$ at $37^{\circ} \mathrm{C}$ in SOC medium shaking at $200 \mathrm{rpm}$. After that the bacteria were plated on agar plates supplemented with antibiotics and incubated over night at $37^{\circ} \mathrm{C}$.

The transformation efficiency was estimated by colony count in the cloned vs. the religated plate. Depending on the estimated ratio, either colonies were picked and grown directly (high ratio) or pre-screened by colony PCR (low ratio). The bacteria were picked into $2.5 \mathrm{ml} \mathrm{LB}+$ antibiotics in working concentration and incubated over night at $37^{\circ} \mathrm{C}$, shaking.

The plasmids were prepped and digested with the enzymes used for cloning and analyzed on an agarose gel. If bands corresponding to insert and vector were detected, the entire coding sequence of the insert was sequenced using sanger sequencing. Verified colonies were frozen in LB $+30 \%$ glycerol at $-80^{\circ} \mathrm{C}$ for further use and are available on request.

\subsubsection{Histology and microsections}

Embryos were fixed in $4 \%$ paraformaldehyde solution overnight, washed 3x with PBS and embedded as previously described (Mateus et al., 2015). Briefly, the embryos were incubated at $4^{\circ} \mathrm{C}$ in $30 \%$ (w/v) sucrose in PBS overnight. The embryos were then incubated for one hour at $37^{\circ} \mathrm{C}$ in in $7.5 \%(\mathrm{w} / \mathrm{v})$ gelatin and mounted afterwards in the same solution. The tissue blocks were frozen in liquid nitrogen cooled isopentane and stored at $-80^{\circ} \mathrm{C}$. The blocks were sectioned using a CM3050S cryostat (Leica, Wetzlar, Germany) and the tissue sections stored at $20^{\circ} \mathrm{C}$.

\subsubsection{Immunohistochemistry}

Immunohistochemistry was performed according to standard protocols, with the following parameters: For wholemount stainings, embryos were fixed overnight in $4 \% \mathrm{PFA}$ at $4^{\circ} \mathrm{C}$. Early embryos ( $\leq 20 \mathrm{hpf}$ ) were dechorionated after fixation, older embryos were dechorionated prior to fixation. Embryos were then permeabilized for $3 \mathrm{~min}$ with $10 \mu \mathrm{g} / \mathrm{ml}$ proteinase $\mathrm{K}$ and blocked in blocking buffer for immunohistochemistry.

For the staining of gelatin sections, the gelatin was removed from the slides by incubation in $\mathrm{PBS}$ for $10 \mathrm{~min}$ at $37^{\circ} \mathrm{C}$. The sections were permeabilized 
for $10 \mathrm{~min}$ at room temperature in permeabilization buffer and blocked in blocking buffer for immunohistochemistry.

After incubation with primary antibodies at $4^{\circ} \mathrm{C}$ over night, I washed wholemount stainings $6 \times 20 \mathrm{~min}$ (6x5 min for slides) and then in incubated with Alexa-fluor conjugated secondary antibodies. DAPI was added to the secondary antibody solution in a concentration of $1 \mu \mathrm{g} / \mathrm{ml}$.

\subsection{7 in situ hybridization}

gata1a probe templates for in situ hybridization experiments were amplified by PCR using primers containing a T7-promoter encoding sequence. An antisense probe labeled with digoxygenin (DIG) was synthesized by in vitro transcription of $200 \mathrm{ng}$ of this template using T7-polymerase and a DIG labelling kit in a $20 \mathrm{\mu l}$ scale according to manufacturer instructions, supplemented with 80 units of RNAsin. The in situ hybridization was performed as previously described in (Thisse and Thisse, 2008).

Embryos were dechorionated and fixed in 4\% PFA over night. After $3 \times 5$ min washing in PBSTween, the embryos were dehydrated by successive washes with 25, 50, 75 and 100\% methanol in PBSTween for 5 minutes each. The embryos were then stored over night or up to 2 weeks in $100 \%$ methanol at $-20^{\circ} \mathrm{C}$.

The dehydrated samples were then rehydrated by successive washes in 75 , 50 and $25 \%$ methanol in PBSTween, followed by $3 \times 5$ min washes in pure PBSTween. After that, embryos were permeabilized for $10 \mathrm{~min}$ using $10 \mu \mathrm{g} / \mathrm{ml}$ proteinase $\mathrm{K}$ in PBSTween. The samples were then postfixed for $20 \mathrm{~min}$ in $4 \%$ PFA to stop the reaction and washed $4 \times 5 \mathrm{~min}$ in PBSTween. Then a prehybridization step in $200 \mu \mathrm{l} \mathrm{HB}(+)$ buffer for $2-5 \mathrm{~h}$ at $65-70^{\circ} \mathrm{C}$ was performed and the buffer then replaced with $200 \mu$ pre-heated $\mathrm{HB}(+)$ buffer containing the antisense probe in a concentration of $1 \mu \mathrm{g} / \mathrm{ml}$. The samples were hybridized over night at $65-70^{\circ} \mathrm{C}$.

The probe was kept for re-use and the hybridization buffer (from now on $\mathrm{HB}(-))$ exchanged with $2 \mathrm{xSSC}$ by successive washes in 75,50 and $25 \% \mathrm{HB}(-)$ in $2 \mathrm{xSSC}$ followed by $2 \mathrm{x} 10$ min washes in $2 \mathrm{x}$ SSC and 2 washes in $0.2 \mathrm{x}$ SSC. All these steps were carried out at $70^{\circ} \mathrm{C}$ and the samples then slowly cooled to room temperature. At room temperature, the $0.2 \mathrm{x}$ SSC was replaced by PBSTween through successive washes of 25, 50, 75 and 100\% PBSTween in 
$0.2 \mathrm{x}$ SSC. The embryos were blocked for 3-4 $\mathrm{h}$ in in situ blocking buffer and incubated with gentle rotation over night in $300 \mu$ 1:5000 anti-DIG ab in in situ blocking buffer at $4^{\circ} \mathrm{C}$.

The samples were washed 6x15 min in PBSTween and then carefully transferred into a 24 well dish for the subsequent washes. After $3 \times 5$ min washes in alkaline Tris buffer, the embryos were stained in the dark using BM purple AP substrate in alkaline Tris buffer. In 15 min intervals, the samples were checked for the staining intensity and the reaction stopped by replacing the staining solution with PBSTween. For imaging, the embryos were placed on an agarose coated dish and imaged using a Nikon SMZ25 binocular microscope.

\subsubsection{Transcriptional profiling}

As the analysis described in this section were in large parts performed by Stefan Günther at the Bioinformatics and Deepsequencing Core Facility of the Max-Planck Instute for Heart and Lung Research, the following sections were written in collaboration with him and do not solely reflect my own work.

\subsubsection{Sample preparation and cell sorting}

Tails from 150 npas4l heterozygous (npas4lbns423/+) and trans-heterozygous (npas4 $l^{\text {bns423/bns297) }}$ mutant embryos were cut at the anterior end of the yolk extension at $20 \mathrm{hpf}$ in DMEM/F10 $+5 \% \mathrm{FBS}+0.01 \%$ tricaine on agarosecoated plates. The cells were dissociated using the Pierce Cardiomyocyte dissociation kit (Thermo Fisher Scientific) according to manufacturer instructions and FACS sorted for GFP-fluorescence using an AriaIII-sorter (BD-Biosciences, San José, California) and DAPI as indicator for dead cells. I thank Kikhi Khrievono and Ann Atzberger from the FACS core facility for technical help with the sorting.

\subsubsection{Bulk RNAseq}

I isolated total RNA using the miRNeasy micro kit including DNase digestion step on the column. $80 \mathrm{ng}$ of total RNA was provided as input material to Stefan Günther at the Bioinformatics core facility. The library preparation, sequencing and basic analyses were performed by him and the data he provided includes DeSeq2-normalized count matrices as well as quality control plots and a basic GO-term analysis. 


\subsubsection{Single cell RNAseq}

The cell suspensions were counted with Moxi cell counter and diluted according to manufacturer's protocol to obtain 5000 single cell data points per sample. Each sample was run separately on a lane in Chromium controller with Chromium Next GEM Single Cell 3' Reagent Kits v3.1. Single cell RNAseq library preparation was done using standard protocol. Sequencing was done on Nextseq500 and raw reads were aligned against the zebrafish genome (DanRer11) and counted by StarSolo (Dobin et al., 2013) followed by secondary analysis in Annotated Data Format. Preprocessed counts were further analyzed using Scanpy (Wolf et al., 2018). Basic cell quality control was conducted by taking the number of detected genes and mitochondrial content into consideration. We removed only 16 cells in total that did not express between 1000 and 7000 genes or had a mitochondrial content less than $6 \%$. Further, we filtered genes if they were detected in less than 30 cells $(<0.3 \%)$. Raw counts per cell were normalized to the median count over all cells and transformed into log space to stabilize variance. We initially reduced dimensionality of the dataset using PCA, retaining 50 principal components. Subsequent steps, like low-dimensional UMAP embedding (McInnes et al., 2018) and cell clustering via community detection (Traag et al., 2019), were based on the initial PCA. Final data visualization was done by scVelo (La Manno et al., 2018; Bergen et al., 2020), and the cellxgene (Megill et al., 2019) package. A trajectory inference was calculated using Partition-based graph abstraction (Wolf et al., 2019) and displayed as force directed graph calculated utilizing ForceAtlas2 (Jacomy et al., 2014).

\subsubsection{Imaging}

\subsubsection{Mounting}

Embryos or $<5$ dpf larvae were anesthetized in $0.01 \%$ tricaine, dechorionated if necessary and transferred into $250 \mathrm{\mu l}$ previously molten $1 \%$ low melting point Agarose ( $0.5 \%$ for light sheet microscopy and overnight time lapses) kept at $42^{\circ} \mathrm{C}$. After careful mixing, the agarose containing the sample was transferred into a glass bottom dish (confocal and spinning disk imaging) or cobweb holder (lightsheet imaging). Using fine forceps, the samples were positioned in the correct orientation. Once the agarose solidified, $2 \mathrm{ml}$ eggwater with $0.01 \%$ tricaine was added into glass bottom dishes to prevent drying. For flat mounted embryos, the yolk syncitial layer was cut in the transverse plane on the opposite side of the embryo and deyolked using 2 fine syringes before flattening it out by placing a cover slip on top of the specimen. 


\subsubsection{Confocal and spinning disk imaging}

Embryos were embedded in 1\% low-melting agarose in eggwater on their side. Living embryos were anaesthetized with $0.01 \%$ tricaine prior to embedding and stayed under anesthesia during the procedure. All experiments on living embryos were non-recovery experiments. For genotyping, the anaesthetized embryos were taken out of the agarose, exposed to heat briefly and lysed using $50 \mathrm{mM} \mathrm{NaOH}$ for $10 \mathrm{~min}$ at $95^{\circ} \mathrm{C}$.

All confocal images were acquired using a LSM800 observer confocal microscope. The images were acquired and processed using the ZenBlue software package. Only linear adjustments were used, acquisition parameters were kept constant throughout the imaging whenever possible. The confocal microscopy data presented in this manuscript were only used for qualitative analyses.

For overview images of the whole embryo, a tile scan with a Pln Apo 10x/0.45 DICII objective was performed and stitched with ESID as reference channel. Brightfield-like images for this magnification were generated using an ESID-channel and enhanced depth of focus. The channel was then added to the orthogonal projection of the fluorescent channels.

Images of the trunk region were acquired using LD LCI Pln Apo 25x $0.8 \mathrm{~W}$ and C Apo 40x/1.1 W DICIII lenses. As an anatomical landmark, I kept the yolk extension in the field of view. For the presentation of the brightfield-like ESID channel, a single plain was exported from the middle of the stack and added to the orthogonal projection of the fluorescent channel.

Sections were imaged using the Pln Apo 40x/1.4 Oil DIC II objective and the Airyscan detector, followed by 2D airyscan processing. Similar-looking transverse sections over the yolk extension were used, but the location along the anterior-posterior axis varies slightly between different sections due to the lack of precise anatomical landmarks.

\subsubsection{Lightsheet imaging}

Early embryos were not dechorionated but embedded with the chorion in $0.5 \%$ agarose with $0.01 \%$ tricaine using a cobweb holder (Strobl et al., 2017) to avoid mechanical stress. The embryo in the chorion was mixed into the agarose and embedded in a minimal volume on the cobweb holder. Imaging from four different angles was used to obtain a 3-dimensional image. 


\subsubsection{Image processing and analysis}

Whenever possible, images were processed directly in Zen and saved in the original format. In some cases, additional linear changes were applied in imageJ. Images from the same experiment were processed in the identical fashion. Fluorescence levels were not quantified in this study.

\subsubsection{Quantification of ISV numbers}

fli1a:GFP - positive ISV were counted under a stereomicroscope at $48 \mathrm{hpf}$ along the entire body axis. Controls and treated embryos derived from the same clutch. All experiments have been repeated at least twice.

\subsubsection{Statistical analyses}

Statistical analyses were coded in R (count and bulk RNAseq data) and python (qPCR and scRNAseq data). Count data was fitted to a Poisson model using the "glm" library. For log-transformed qPCR data, normality was assumed, and p values calculated by unpaired two-sample ttest using the "scipy" package. Bonferroni correction was applied to adjust p values, when appropriate. 


\section{Chapter 4}

\section{Results}

\subsection{Targeting and knock-in strategies}

The almost complete lack of endothelial cells in homozygous cloche/npas $4 \mathrm{l}$ mutants is well known and extensively studied as it is the strongest vascular phenotype observed in vertebrates (Stainier et al., 1995; Liao et al., 1997; Thompson et al., 1998; Parker and Stainier, 1999). However, the gene responsible for this phenotype was not discovered until recently (Reischauer et al., 2016). For that reason, transgenic tools such as reporter lines, overexpression lines or other tools had not been generated thus far.

When I started working on npas 4 l, the most interesting questions related to it included potential orthologues in mammals, potential upstream candidates, potential interaction partners and visualizing the gene or protein itself. I decided to generate a V5-tagged line, that would allow the detection of Npas4l on the protein level. To address questions concerning the subcellular localization or the members in a protein complex, the tagged Npas4l-V5 protein had to retain its protein function. Three attempts to generate such a tagged line achieved knocking in a V5 tag precisely into coding sequences with relatively low sequence conservation as suggested by Burg et al. (2016) but all of these insertions impaired the protein function in the process (Figure 4.1, unpublished results).

Analyzing the previous V5-tagging attempts and various published mutants, I noticed that the strength of the phenotype caused by mutations or knock-ins into the npas $4 l$ locus decreased towards the 3'-end of the coding sequence: Almost no endothelial and a complete lack of endocardial cells were observed in mutants affecting the 5' -region such as bns297 or s5, but a milder 
defect was detected in the bns59 allele that affected the 3'-region (Reischauer et al., 2016). Moreover, an overexpression reagent used to in vitro transcribe npas4l-HA mRNA containing a flexible linker and an HA-tag at the 3'-end of the coding sequence had been generated before and showed biological activity (Marass et al., 2019). I hypothesized that an insertion close to the 3 '-end of the endogenous coding sequence allows the knock-in of a small V5 tag without destroying the gene function. To increase the chance of success, I also inserted the flexible linker sequence previously used successfully to tag Npas4l with an HA-tag (Marass et al., 2019).

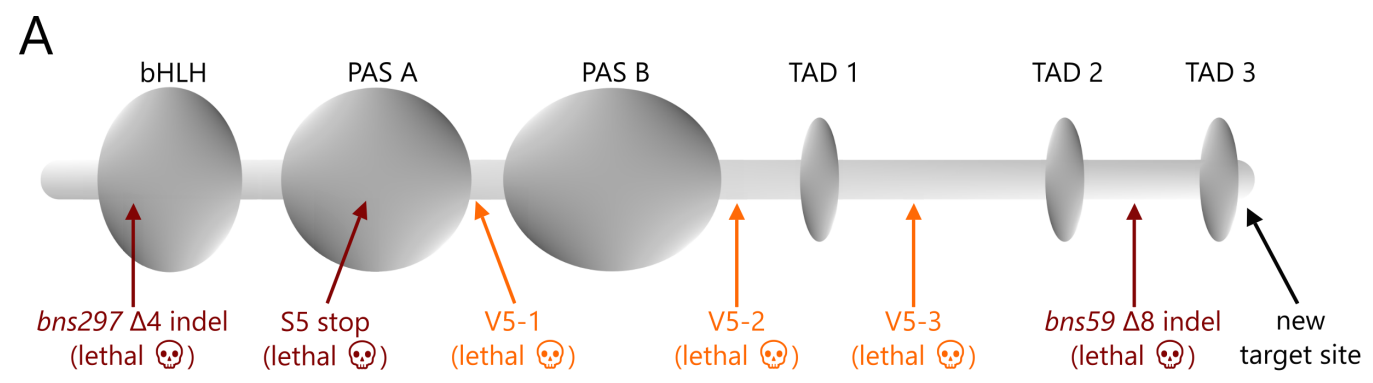

relative strength of phenotype

B

$\stackrel{\text { knock-in }}{\longrightarrow}$

tagged npas4I

DNODOC

npas 41
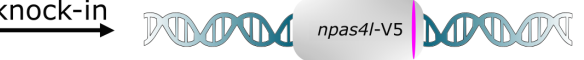

knock-in

npas $4 I^{+}$reporter

npas4I-p2A-Gal4-VP16

$\stackrel{\text { knock-out }}{\longrightarrow}$

indel npas4I- reporter

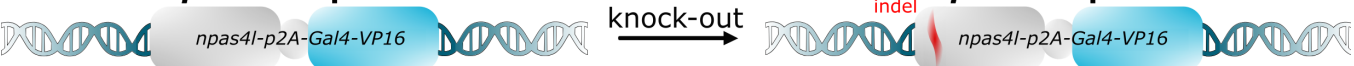

Figure 4.1: Generation and use of a knock-in reporter to trace npas4l expressing cells in wild-type and mutant embryos. (A) Schematic representation of the Npas4l peptide. Known mutant alleles highlighted in brown and known V5-insertions highlighted in orange. Although all known alterations to the locus are lethal, phenotypical strength decreases towards the sequences encoding the C-terminus of the peptide. Knock-ins that should retain the gene function should therefore be targeted to the 3'-end of the coding sequence. (B) Two knock-ins are to be inserted into the chosen target site; A linker-V5 insertion and a -p2a-Gal4-VP16. Subsequently, if this strategy retains Npas4l function, the DNA-binding site of the Gal4-reporter allele can be altered to knock-out the gene and analyze reporter expression in npas $4 l$ mutant embryos.

Inserting a small V5 tag was a strategy I tested several times already, so I wanted to use this opportunity to insert a larger reporter cassette such as GFP or Gal4. To this end, I decided to use the same targeting reagents 
combined with a new insertion method shared with us by Jeffrey Essner's lab ahead of publication, now published in Wierson et al. (2020). This strategy relied on the same targeting reagents already optimized for the V5 tagging. It aims to generate a fluorescent reporter line as a kind of targeted Genetrap approach in which a loss of function and a fluorescent reporter are combined to allow tracing of cells that express the mutated gene in heterozygous and mutant animals. As the targeting strategy prioritizes retaining Npas4l function and the target site was chosen to minimize the chances to disrupt the protein function, it was possible to get a Gal4 reporter allele that would not lead to a loss of Npas4l function. In this case, a mutation in the DNAbinding domain encoding region could be introduced in a second step to get the desired allele. A graphic representation of both strategies is depicted in figure 4.1B. A comparison of the two approaches as well as strengths and limitations are summarized in table 4.1.

Table 4.1: Comparison between V5-tagging and generation of a Gal4 reporter line for npas 4 l. The two approaches complement each other and were therefore pursued simultaneously.

\begin{tabular}{l|l} 
V5-tag & Gal4-VP16 reporter \\
\hline+ subcellular resolution & + signal amplification \\
+ proteomics/pulldowns possible & + driver line/high flexibility \\
+/- endogenous protein levels & + live imaging possible \\
- might be present transiently & + /- stable reporter signal \\
- Npas4l needs to remain functional & - line might be silenced / lethal \\
- no live imaging possible & - low spatiotemporal resolution
\end{tabular}

To knock-in both donors, I used CRISPR/Cas9 mediated genome editing to induce a double strand break in the last exon of the npas $4 l$ coding sequence and provided repair templates with short homology arms to facilitate insertion by homology directed repair (Burg et al., 2016; Wierson et al., 2020). In a first step, guide RNA's were designed and tested for efficiency (Figure 4.2A). A major problem turned out to be targeting sequences close to the npas 4 l 3'-UTR, which contains highly repetitive sequences and cannot be amplified easily by PCR. Therefore, a guide RNA sequence cutting $67 \mathrm{bp}$ upstream of the stop codon was designed that allowed placing primers for efficiency testing into the coding sequence and avoiding repetitive regions.

In the second step, I designed the knock-in templates and tested the integration efficiency. For the Gal4 knock-in, I used a template vector linearized in vivo by a universal guide sequence, resulting in a linear double 
stranded template with 48 bp homology arms on both sides. The efficiency of the knock-in was estimated by injecting the reagents into the $U A S$ :GFP background and screening for mosaic fluorescence (Figure 4.2B). Some fish with very strong mosaicism were found verifying the general efficiency of the knock-in strategy. On top of a Gal4-reporter, I also tried to generate a GFP and a nuclear GFP line, but preliminary analyses indicated a very low fluorescence in the injected fish (data not shown, founder animals in the injected P0 embryos have not been found for these constructs). To ease the screening, I only raised injected embryos with at least one GFP-positive cell as this ensured the presence of $U A S$ :GFP in the background, making sure that all in-crosses or out-crosses of potential founders could produce GFPpositive progeny and could, therefore, be identified as founders based on their reporter expression.

A
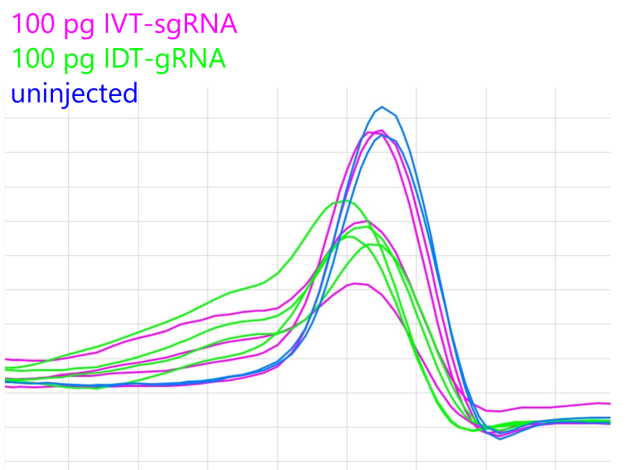

C

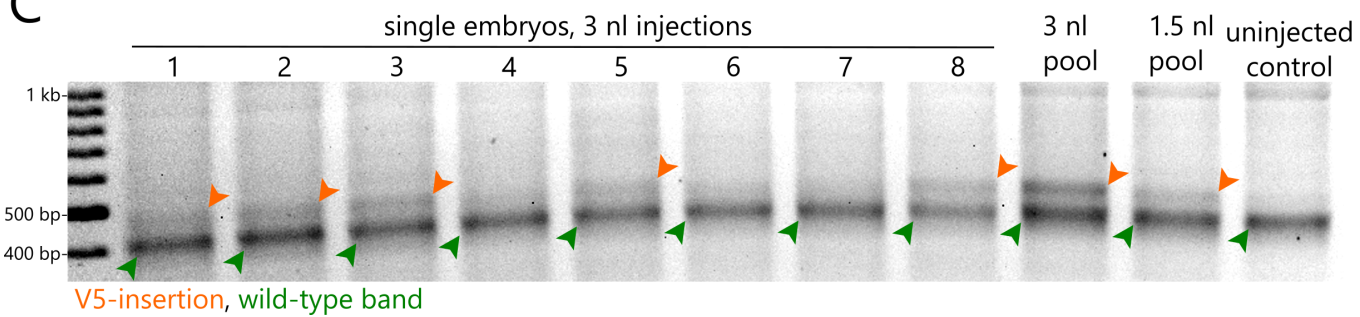

B

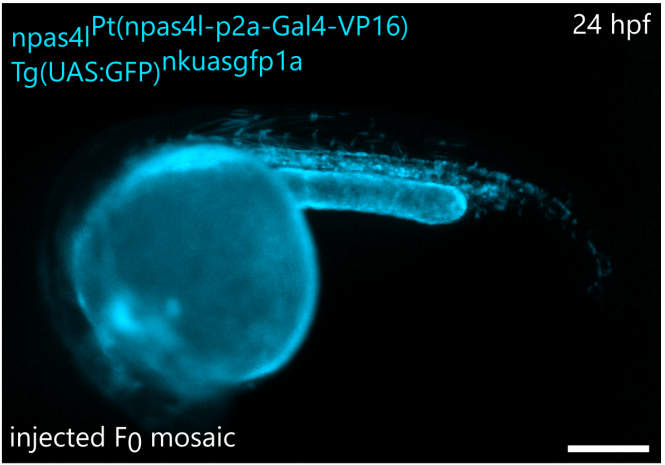

Figure 4.2: A working targeting strategy to knock-in a V5-tag or a Gal4 reporter into the endogenous npas 4 l locus. (A) High resolution melt analysis of uninjected control embryos (blue) and embryos either injected with in vitro transcribed single guide RNA (pink) or synthetic guide RNA duplexes (green). Synthetic guide RNA duplexes appeared to be slightly more efficient and were used to knock-in Gal4 and V5. (B) Fluorescence in the yolk, endothelial cells and some anterior muscles was detected in mosaic P0 embryos validating the Gal4-targeting strategy. (C) PCR with flanking primers to detect V5-insertion in injected P0 embryos. The relative strength of the V5 compared to the wild-type band can be used to estimate the knock-in efficiency semi-quantitatively and serves as a validation for the knock-in approach. Scale bar: $200 \mu \mathrm{m}$. 
For the V5 knock-in, I used a single-stranded oligo template and tested the efficiency by PCR that flanked the insertion, allowing to see the knockin as an upward shift in the PCR band and to assess the efficiency semiquantitatively (Figure 4.2C). The conditions were optimized, and P0 animals grown for both approaches. The founder screening, line generation and initial characterization of these reporters will be described in detail in the next sections.

\subsection{Generation of the npas4l-V5 $5^{\text {bns314 }}$ allele}

I started to screen the injected P0 fish by conventional in-crossing of the founders and testing for the insertion by insert-specific PCR on three pools of $20 \mathrm{~F} 1$ embryos each. Two founder couples were found using this method (screening results summarized in table 4.2). Sanger sequencing of the PCR products confirmed a complete V5 insertion, but showed indels in the 3'insertion sites, which was not optimal given the goal of generating an npas $4 \mathrm{l}$ allele with intact Npas4l function that could be used for experiments such as pull-downs.

Table 4.2: Screening outcome for V5-tagging of Npas4l. Founder \#23 (green) was found to carry a precise insertion on both ends verified by Sanger sequencing. The screening was stopped once this result was obtained and hence some insertions were not analyzed (n.a.). indel: fragment shorter or longer as determined by PCR. No sequence validation. "precise" or the deletion size indicate that the allele has been sequenced. F: founder screened by sperm squeezing, FC: founder cross found through conventional in-crosses.

\begin{tabular}{l|l|l} 
Founder & 5'-insertion & 3'-insertion \\
\hline FC\#1 & precise & -4 bp \\
FC\#5 & precise & indel \\
F\#8 & precise & n.a. \\
F\#18 & -19bp & n.a. \\
F\#21 & precise & n.a. \\
F\#23 & precise & precise \\
F\#24 & precise & n.a. \\
F\#31 & precise & n.a. \\
F\#32 & indel & n.a.
\end{tabular}

To speed up the screening, I tested sperm squeezing of adult males (Lebcir et al., 2016) to pre-screen a large number of fish for germline transmission and then set up outcrosses of the founders determined to be positive for the insertion by this method. I found the male founder fish \#23 to carry a precise 
insertion of both the 5' and 3' ends as determined by PCR with flanking primers (Figure 4.3A). Progeny of this founder was used to generate the tagged V5 allele npas4l-V5bns314.

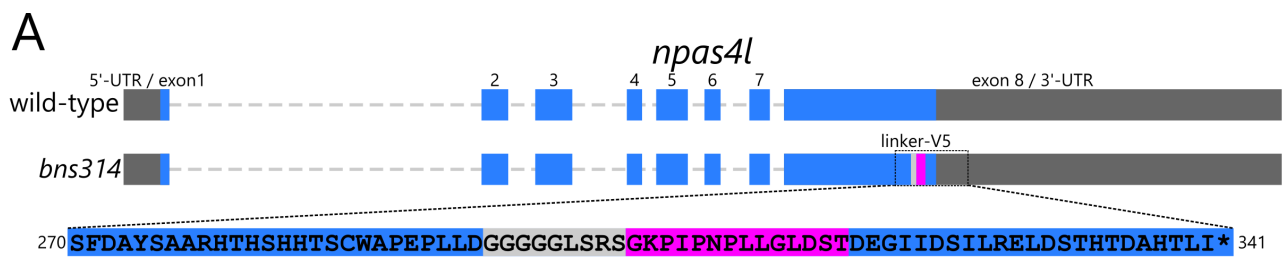

\section{B}
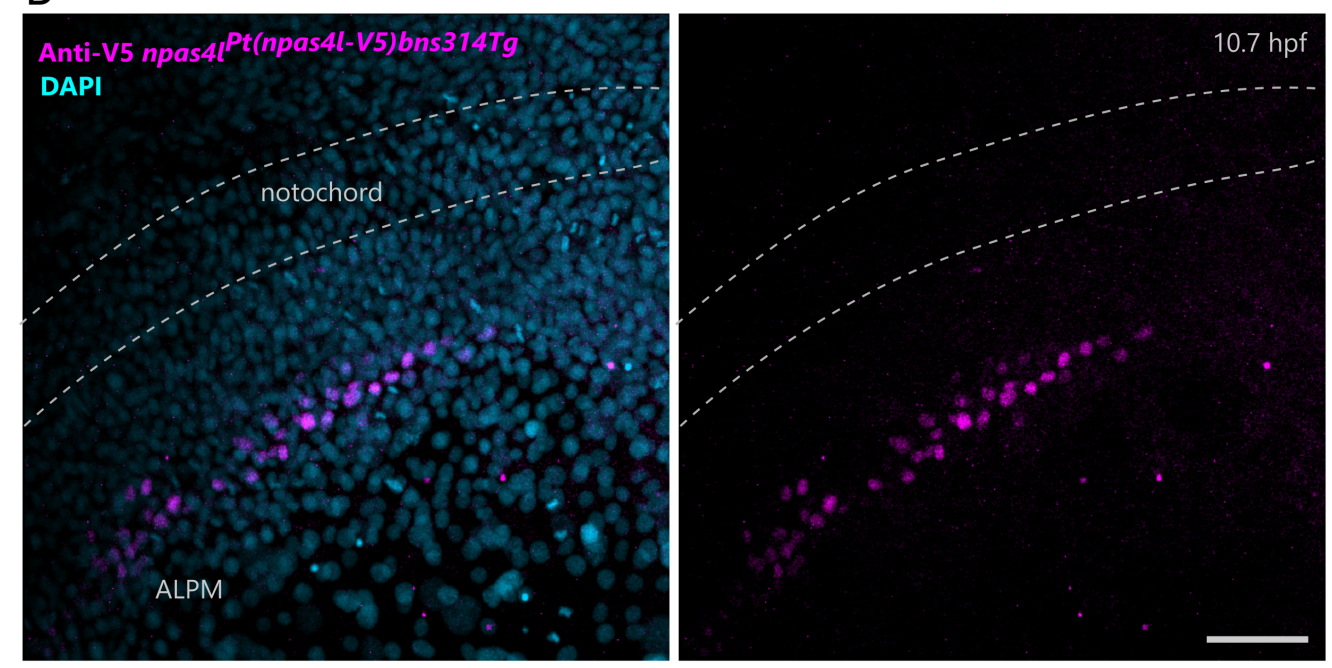

C

D

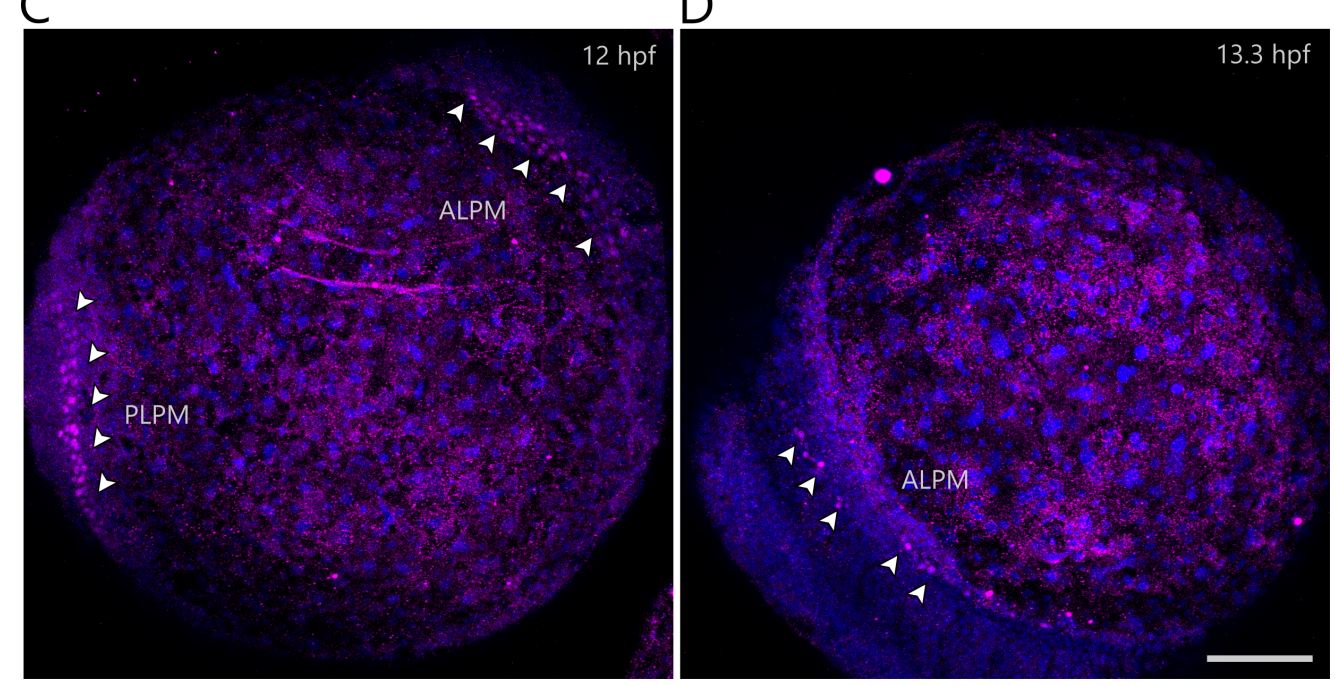

Figure 4.3: A nuclear V5 signal can be detected on the lateral side of the Npas4l-V5 positive embryos during early somite stages. Figure legend continued on the next page. 
Figure 4.3: (A) Structure of the endogenous locus in wild-type and linker-V5 insertion including the amino acid sequence spanning the insertion site. (B) Immunostaining against V5 in Npas4l-V5 positive embryos at $10.7 \mathrm{hpf}$ (2 somite stage) co-localizes with DAPI at the lateral site of the embryo. The notochord has been highlighted as landmark. The signal can be detected at the (C) $12 \mathrm{hpf}$ (6 somite stage) and (D) $13.3 \mathrm{hpf}$ (10 somite stage) as well (arrowheads). Areas of V5 detection at all stages correspond to the presumed anterior lateral plate mesoderm (ALPM) and posterior lateral plate mesoderm (PLPM). Scalebars: $50 \mu \mathrm{m}(\mathrm{B}), 100 \mu \mathrm{m}$ (C-D).

\subsection{Npas4l-V5 is detectable on the protein level during early embryonic development}

Next, I tested whether Npas4l-V5 is detectable on the protein level. As Npas4l is an early transcription factor expressed in the angioblasts located in the lateral plate mesoderm, I expected detecting a lateral nuclear Npas4lV5 protein in late gastrulation or early somite stage embryos.

Wholemount immunohistochemistry against the V5-tag resulted in such a signal at 10.7, 12 and 13.3 hpf (Figure 4.3B-D). The staining was weak but the signal clearly nuclear and lateral as expected (Figure 4.3B). Positive embryos were confirmed to carry the V5 tag by genotyping the positive fish (data not shown). However, often many more cells were detected on one side of the embryo compared to the other. To visualize this effect, I flatmounted some of the embryos for imaging (Figure 4.4A). Through this analysis, I confirmed that although some cells on both sides can be observed to be positive for Npas4l-V5 staining, they are concentrated to one side of the anterior lateral plate mesoderm at this stage.

Thinking about potential reasons for the observed effect, I hypothesized that this might be caused by the instability of the tagged protein. Therefore, I used a prediction algorithm for potential PEST sequences in the peptide, that may lead to instability of Npas4l. The Emboss epestfind software (Rice et al., 2000) predicts a 30 amino acids long strong PEST candidate located between the PAS and TAD domains and spanning the sequence from R340 to H370 (Figure 4.4B). Interestingly, this sequence is a repetitive motif on the DNA level that appears to show a length polymorphism in different laboratory strains (data not shown).

Despite the apparently low Npas4l-V5 protein abundance, I attempted to detect the V5-Tag in western blots. However, I was not able to detect its 

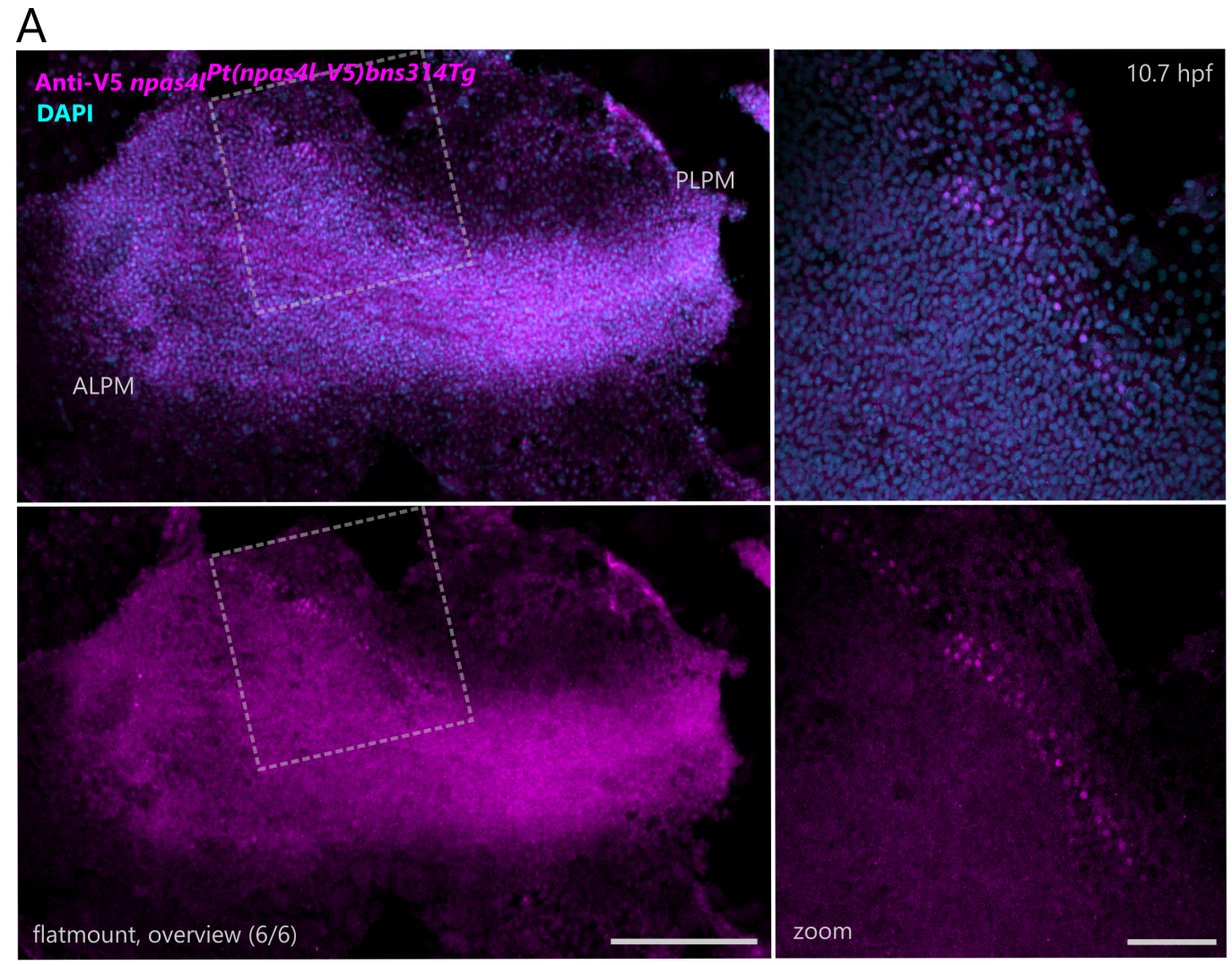

B

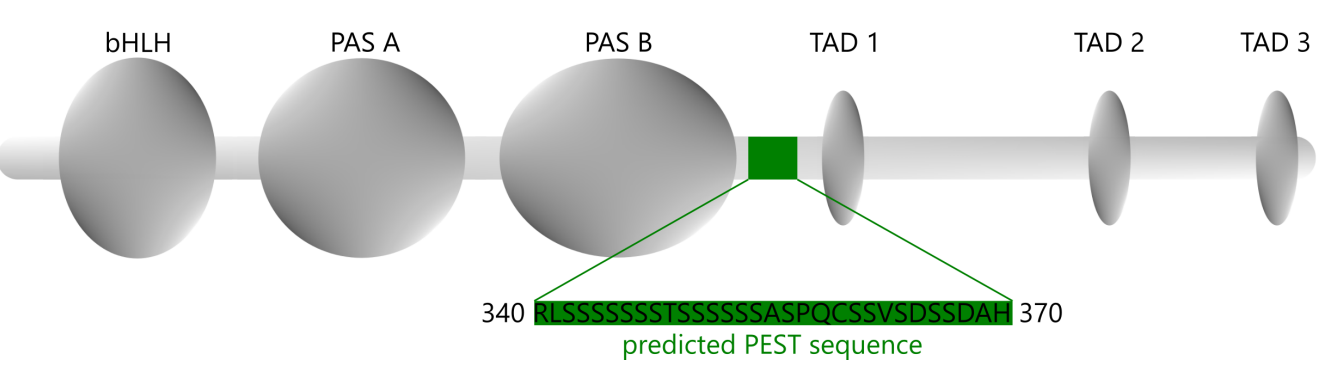

Figure 4.4: The Npas4l-V5 epitope is often detected unilaterally, which might reflect protein instability. (A) Flatmounted wholemount immunohistochemistry against V5 in Npas4l-V5 positive embryos at $10.7 \mathrm{hpf}$ (2 somite stage) shows colocalization of the signal with the lateral plate mesoderm. The signal was consistently observed more on one side than the other suggesting that the presence of Npas4l could be transient. (B) The prediction software epestfind (https://emboss.bioinformatics.nl/cgibin/emboss/epestfind) indicates that a repetitive region in the middle of the Npas4l peptide sequence may be a PEST motif that could lead to a fast proteasomal degradation of the peptide. Scale bars: $200 \mu \mathrm{m}$ (overview), $50 \mu \mathrm{m}$ (zoom). 
presence in deyolked embryo lysates and nuclear extracts (data not shown). Enrichment of the protein by pull-down from a large sample may allow not only the detection in a western blot but also the analysis of these lysates by mass spectrometry. As it is not a straightforward project and would require some time and optimizations, I decided not to pursue this line of investigation for this thesis as the analysis of the Gal4 reporter alleles appeared to be the more promising direction at this point.

\subsection{Generation of the npas $4 l^{\text {bns313 }}$ reporter allele}

For the founder screening, $U A S$ :GFP positive injected $\mathrm{P} 0$ fish were incrossed and the F1 embryos screened for fluorescence. Out of 54 fish that were screened, three transmitted the Gal4 reporter allele (Table 4.3). Although all these founders displayed indistinguishable expression patterns, only $2 / 3$ transmitted a precise 5'-insertion of the p2a-Gal4 (Figure 4.5A and B). I chose to work with the allele transmitted by founder \#3.

Table 4.3: Screening outcome for Gal4-VP16 tagging of Npas4l. The male of founder cross \#3 (green) carried a precise 5'-insertion of the cassette. More alleles were screened for later and kept as back up alleles. A total of 54 fish have been screened.

\begin{tabular}{l|l|l} 
Founder & 5'-insertion & 3'-insertion \\
\hline FC\#3 & precise & n.a. \\
FC\#11 & precise & n.a. \\
F\#25 & +45 bp & n.a.
\end{tabular}

However, I could not resolve the 3'-end of the cassette by PCR. Therefore, I tested the allele for the presence of the vector backbone and concatemeric insertions by PCR. These tests were positive for all three founders suggesting that the unspecific insertion of the vector backbone and concatemers were present in all founders. As the reporter cassette carries its own betaActin PolyA sequence (Figure 4.5A) and, therefore, terminates the sequence, I decided to keep the presence of 3'-vector insertions in mind but concluded that they do not necessarily influence the expression or protein function. 


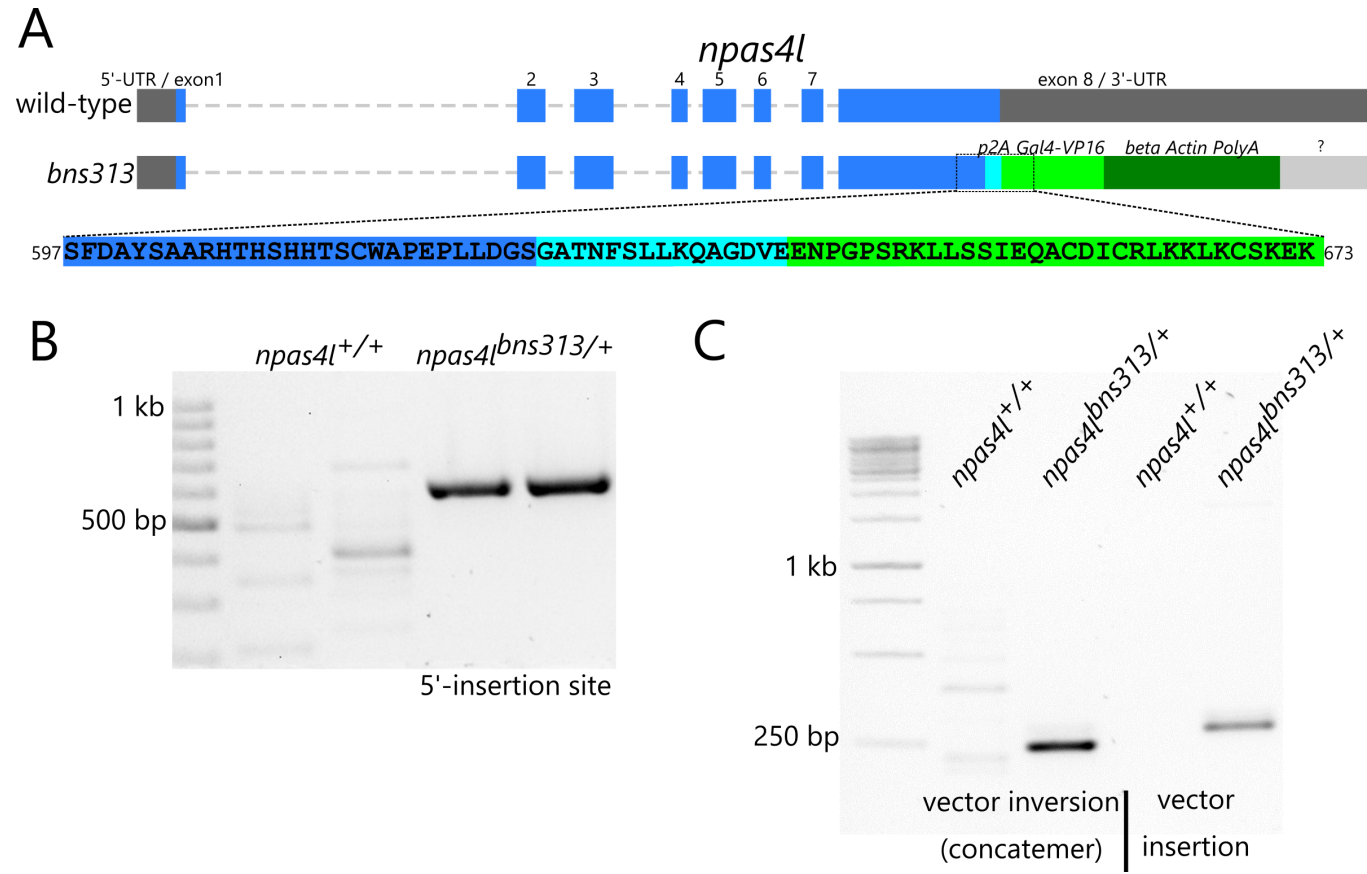

Figure 4.5: Generation and use of a knock-in reporter to trace npas $4 l$ expressing cells in wild-type and mutant embryos. (A) Structure of the endogenous locus in wild-type and -p2a-Gal4-VP16 insertion including the amino acid sequence spanning the insertion site. (B) The presence of the Gal4-reporter cassette can be cleanly verified by PCR. (C) Presence of tail-to-tail vector concatemers in the reporter allele, indicating the presence of at least two cassettes.

By the time I discovered the presence of vector sequences, I had already generated a mutated variant of the reporter allele (compare to section 4.9) and decided to test those fish that carry the same reporter allele on the cDNA and gDNA levels for correct splicing and genomic aberrations. These data are displayed and discussed in figure $4.17 \mathrm{C}$ and $\mathrm{D}$ and are equally valid for the bns313 allele.

\section{5 npas 4 l reporter positive cells can be de- tected in endothelial cells from early so- mite stages onwards}

In early somite stages such as $10.7 \mathrm{hpf}$, npas $4 \mathrm{l}$ reporter positive cells were detected on the ventral lateral side of the embryo as well as in the yolk syncytial layer (Figure 4.6A). In all subsequent embryonic and larval stages observed up to five days post fertilization (dpf), the reporter fluorescence was 
detected (Figure 4.6 B-D).

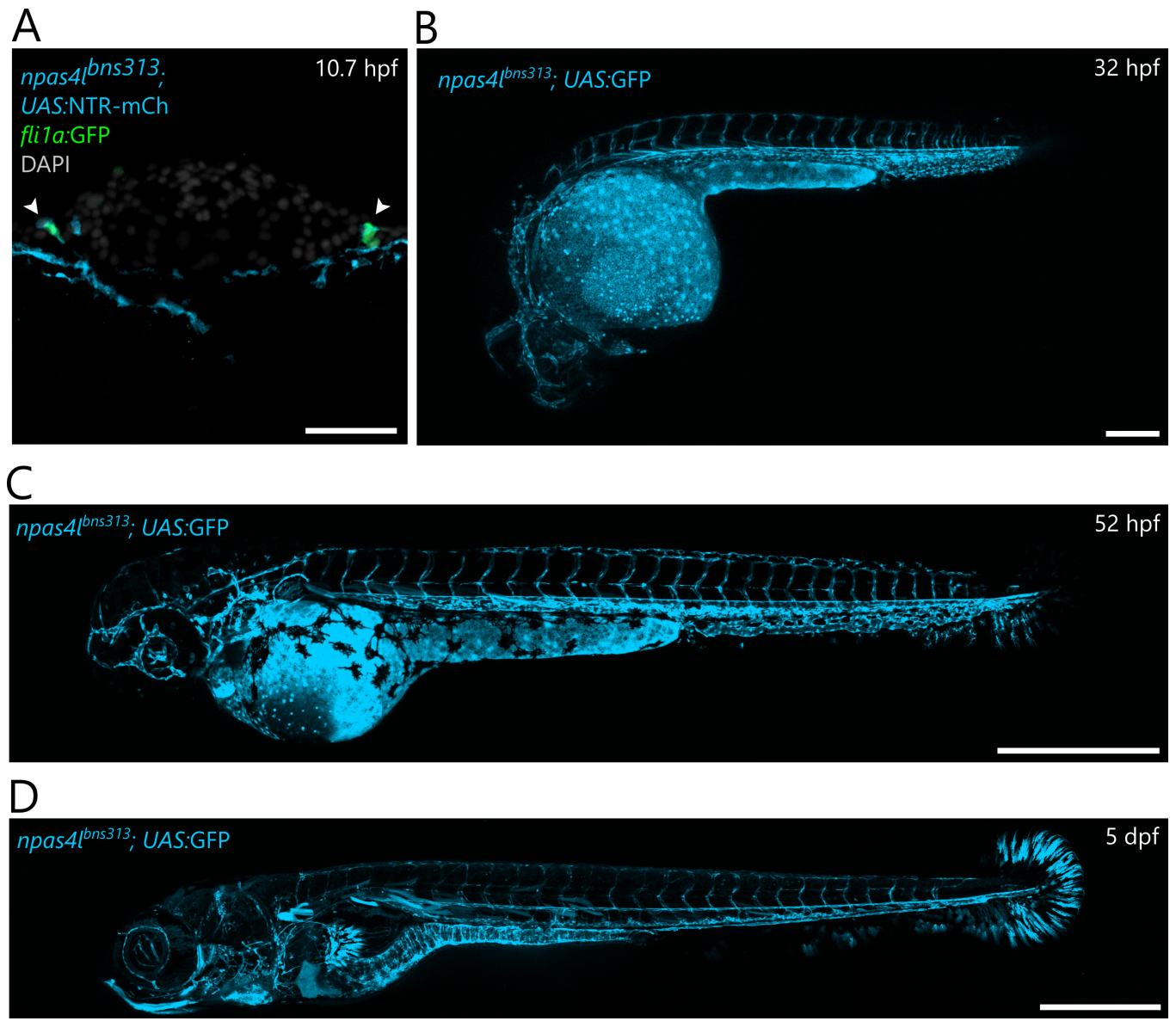

Figure 4.6: The npas 4 l reporter allele is expressed in endothelial cells beginning at early somite stages. (A) npas $4 \mathrm{l}$ and fli1a reporter double positive endothelial progenitors (arrowheads) on the lateral side of the $10.7 \mathrm{hpf}$ (2 somite stage) embryo. (B-D) npas $4 \mathrm{l}$ reporter expression is detected at later stages such as $32 \mathrm{hpf}(\mathrm{B}), 52 \mathrm{hpf}(\mathrm{C})$ and 5 dpf (D) throughout the embryo. Endothelial cells, the yolk syncytial layer, cardiomyocytes, skeletal muscle and some cells in the tail are positive for the reporter fluorescence. Scale bars: $50 \mu \mathrm{m}(\mathrm{A}), 200 \mu \mathrm{m}(\mathrm{B}), 500 \mu \mathrm{m}$ (C-D).

Gal4-VP16 causes a strong amplification of signals and is itself very stable. At the same time, the translation and folding of the Gal4 precedes GFP expression causing a time delay between npas $4 l$ expression and GFP reporter fluorescence. Therefore, it is not possible to say whether npas $4 l$ is still expressed in reporter positive cells or has been expressed earlier and residual GFP reporter expression is still observable. In turn, an npas4l positive cell may not show GFP signal yet due to the inherent delay caused by the Gal4/UAS system. Nevertheless, the stability of the signal allows the 
tracing of very weak and transient expression for a long time, which was the main rationale for generating this line.
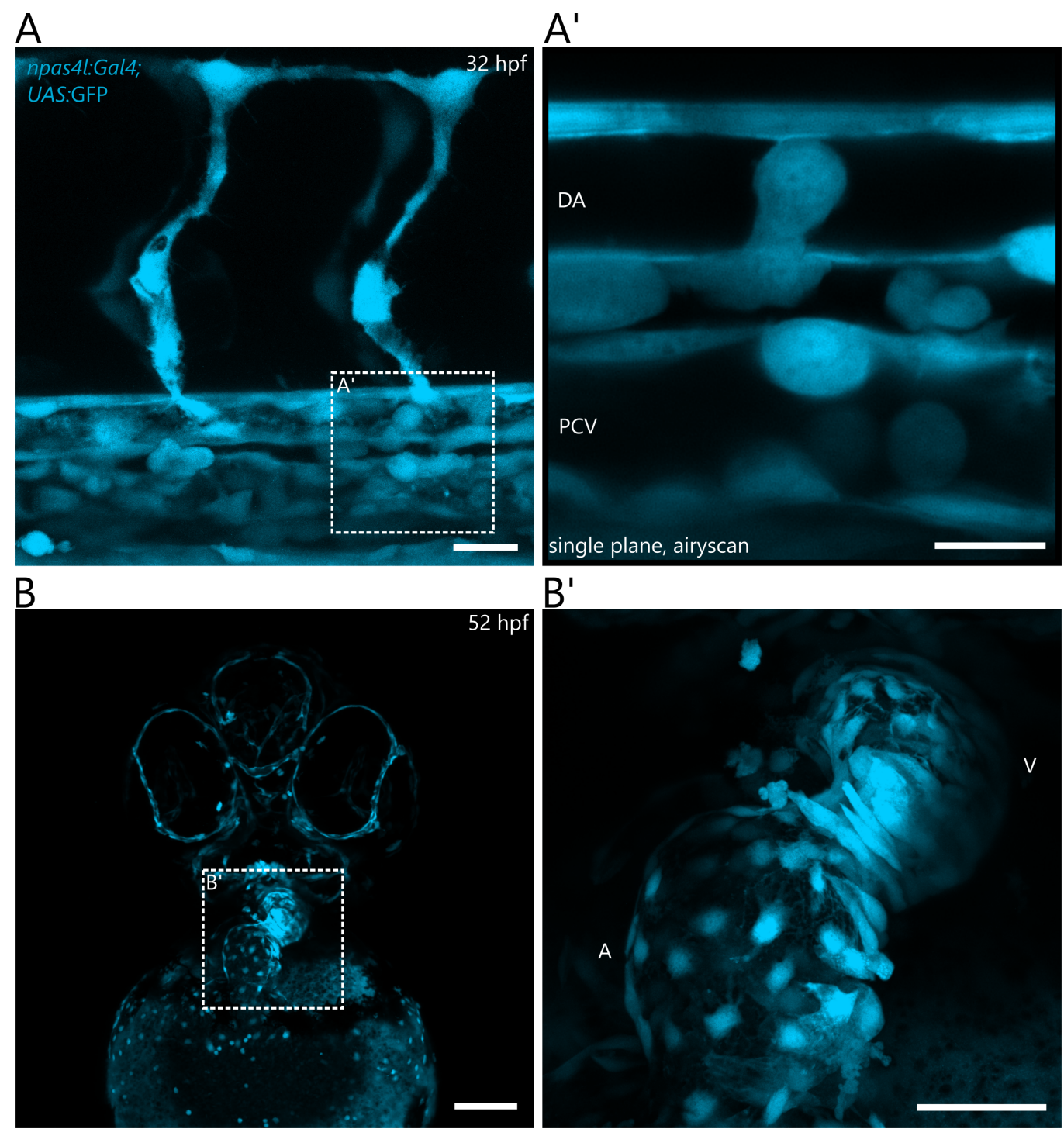

Figure 4.7: npas 4 l reporter expression patterns in wild-type embryos. (A-A') npas $4 \mathrm{l}$ reporter expression in trunk endothelium and circulating blood cells at $32 \mathrm{hpf}$. (BB') npas $4 l$ reporter expression in the heart. DA: dorsal aorta, PCV: posterior cardinal vein, A: atrium, V: ventricle. Scale bars: $20 \mu \mathrm{m}$ (A), $10 \mu \mathrm{m}$ (A'), $100 \mu \mathrm{m}$ (B), $50 \mu \mathrm{m}$ (B').

Expression was observed in endothelial cells (Figure 4.7A), blood (Figure 4.7A') as well as in the endocardium and few cardiomyocytes of the embryonic heart (Figure 4.7B'). The signal in blood cells faded quickly and became very weak by $48 \mathrm{hpf}$ (data not shown). Also, a signal in the yolk 
syncytial layer (Figure 4.6) and some skeletal muscles (Figure 4.6B-D) could be observed. Beginning at $48 \mathrm{hpf}$, cells in the pectoral fin buds and tail fin presented positive for the npas 4 l reporter (Figure 4.6 C and D). At $5 \mathrm{dpf}$, the signal in endothelial cells was fading, but a strong fluorescence in the jaw muscles was detected (Figure 4.6D).

To analyze whether all blood endothelial cells express GFP-fluorescence, I imaged the npas $4 l$ reporter in the $k d r l: T a g B F P$ transgenic background. All endothelial cells observed at $52 \mathrm{hpf}$ expressed both reporters (Figure 4.8). I conclude that the npas $4 l$ reporter labels most or all endothelial cells during early embryonic development.
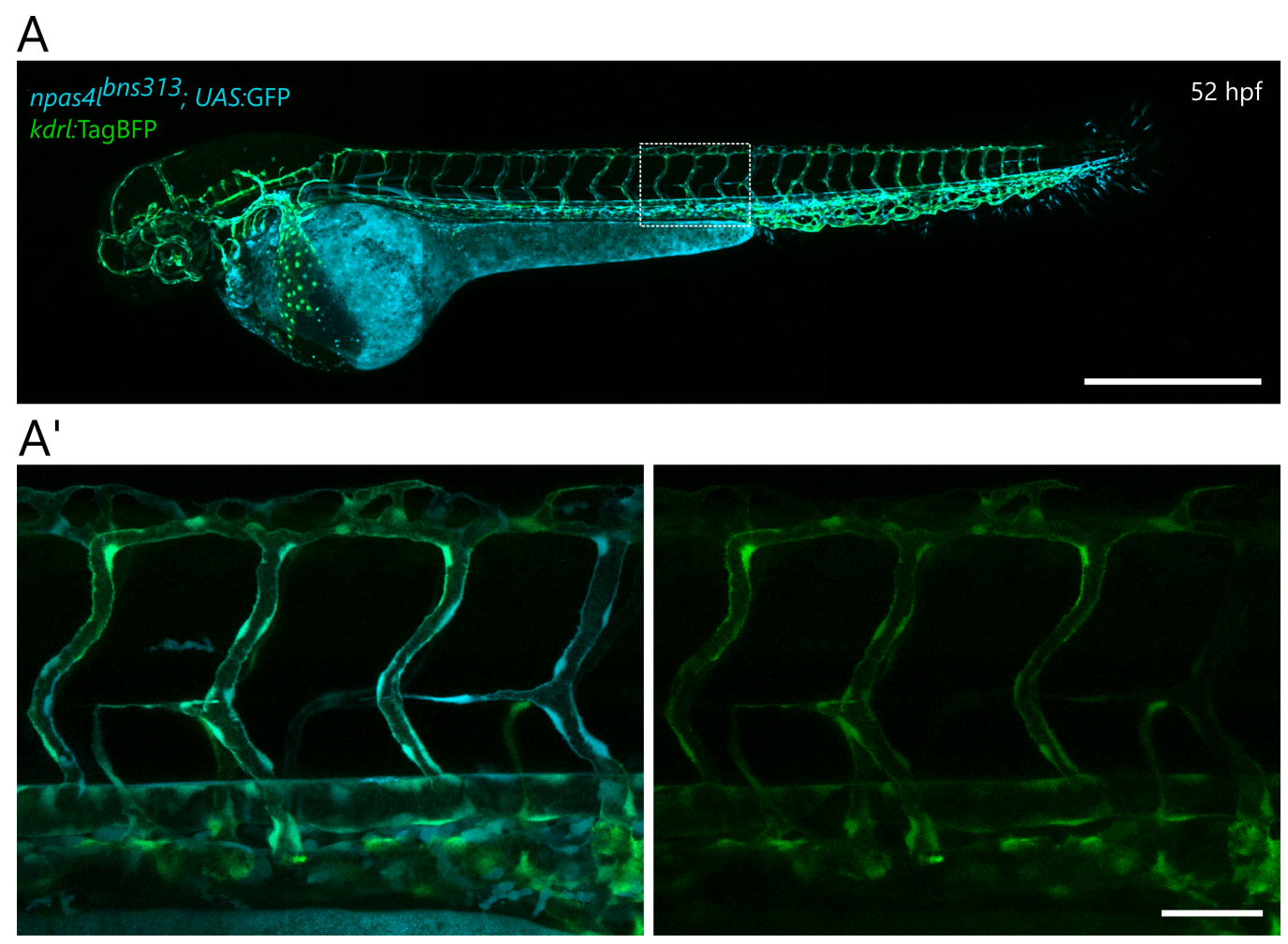

Figure 4.8: The npas $4 l$ reporter is expressed in all $k d r l$ reporter positive endothelial cells at $\mathbf{5 2} \mathbf{~ h p f . ~ O v e r l a p p i n g ~ s i g n a l ~ o f ~ t h e ~ t w o ~ r e p o r t e r s ~ c a n ~ b e ~ s e e n ~ t h r o u g h o u t ~}$ the embryo. Scalebars: $500 \mu \mathrm{m}(\mathrm{A}), 50 \mu \mathrm{m}\left(\mathrm{A}^{\prime}\right)$.

The reporter signal in some cardiomyocytes was unexpected. To analyze whether the npas $4 l$ positive cardiomyocytes might represent a subset of immature cardiomyocytes, I used the transgenic marker gene tnnc2 (Ayele Tadesse Tsedeke, unpublished). However, I did not see a strong correlation between tnnc2 and npas 4 l reporter expression refuting this hypothesis 
(Figure 4.9). In fact, the cardiomyocytes showing the strongest positive signal for the tnnc2 reporter located in the region of the ventricle close to the outflow tract were negative for npas 4 l. As cells in this region are usually derived from the second heart field, it might be that the npas $4 l$ reporter is expressed in cardiomyocytes derived from the first but not from the second heart field. Imaging of early stages before $24 \mathrm{hpf}$ would be necessary to investigate this hypothesis. If all cardiomyocytes at this stage would be npas $4 l$ reporter positive, this would be a strong indication for the reporter being a first heart field marker.
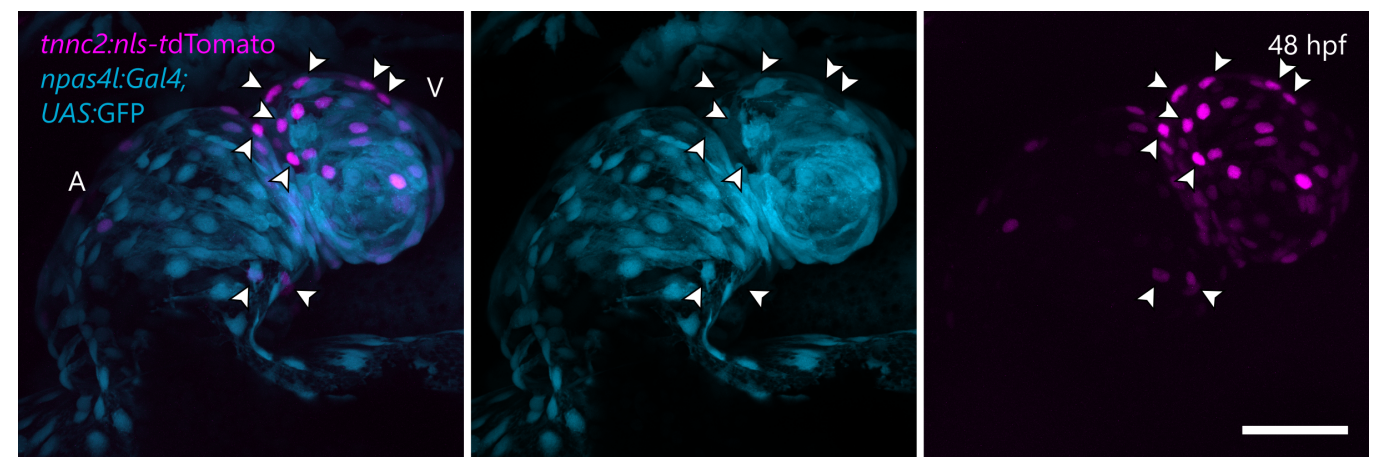

Figure 4.9: The npas 4 l reporter does not co-localize with the immature cardiomyocyte marker tnnc2 in all cardiomyocytes. tnnc2 reporter positive cardiomyocytes that are negative for the npas 4 l reporter are concentrated close to the bulbus arteriosus (npas 4 l reporter negative cardiomyocytes highlighted by arrowheads). Scalebar: $50 \mu \mathrm{m}$.

\section{6 npas 4 l reporter expression in adult ze- brafish}

To investigate the expression of the npas 4 l reporter during adult stages, I sacrificed one male animal and looked for npas 4 l reporter positive organs and tissues. Surprisingly, a very strong GFP signal was visible even through the skull under a dissection microscope. I, therefore, dissected and imaged the brain and determined that the brain structures exhibiting GFP fluorescence were the optic tectum and the cerebellum (Figure 4.10A). Coronal sections through the optic tectum showed that the GFP signal derived from the neuropil layer as well as from the torus longitudinalis (Figure 4.10A'). npas $4 l$ reporter expression in brain vascular structures was not observed. 

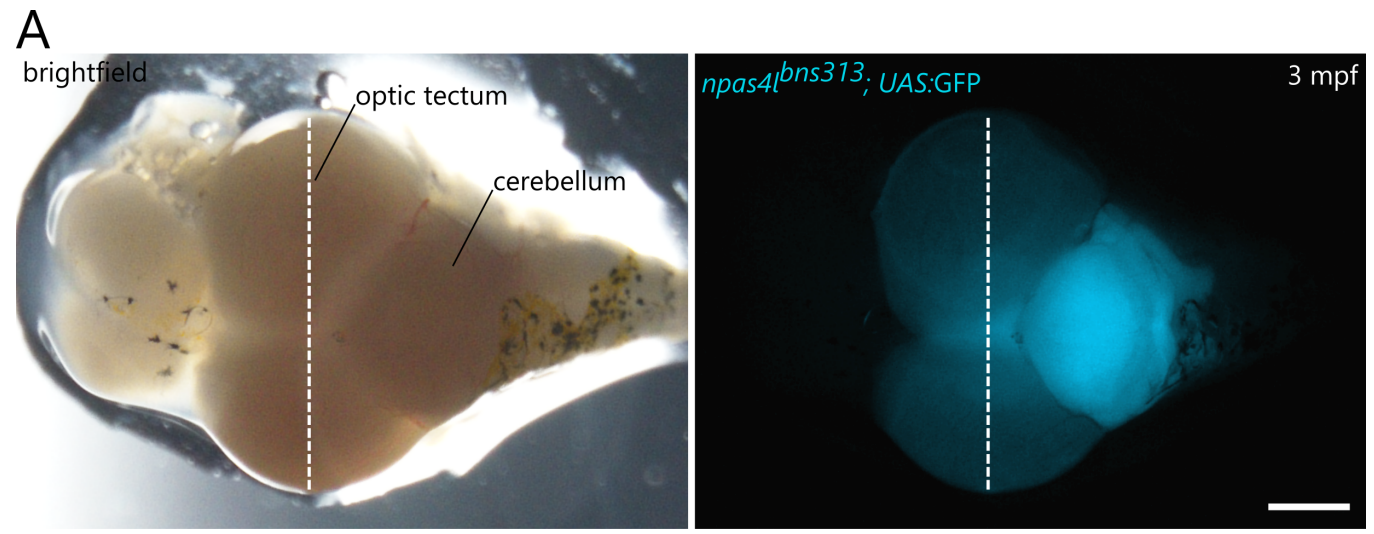

$A^{\prime}$

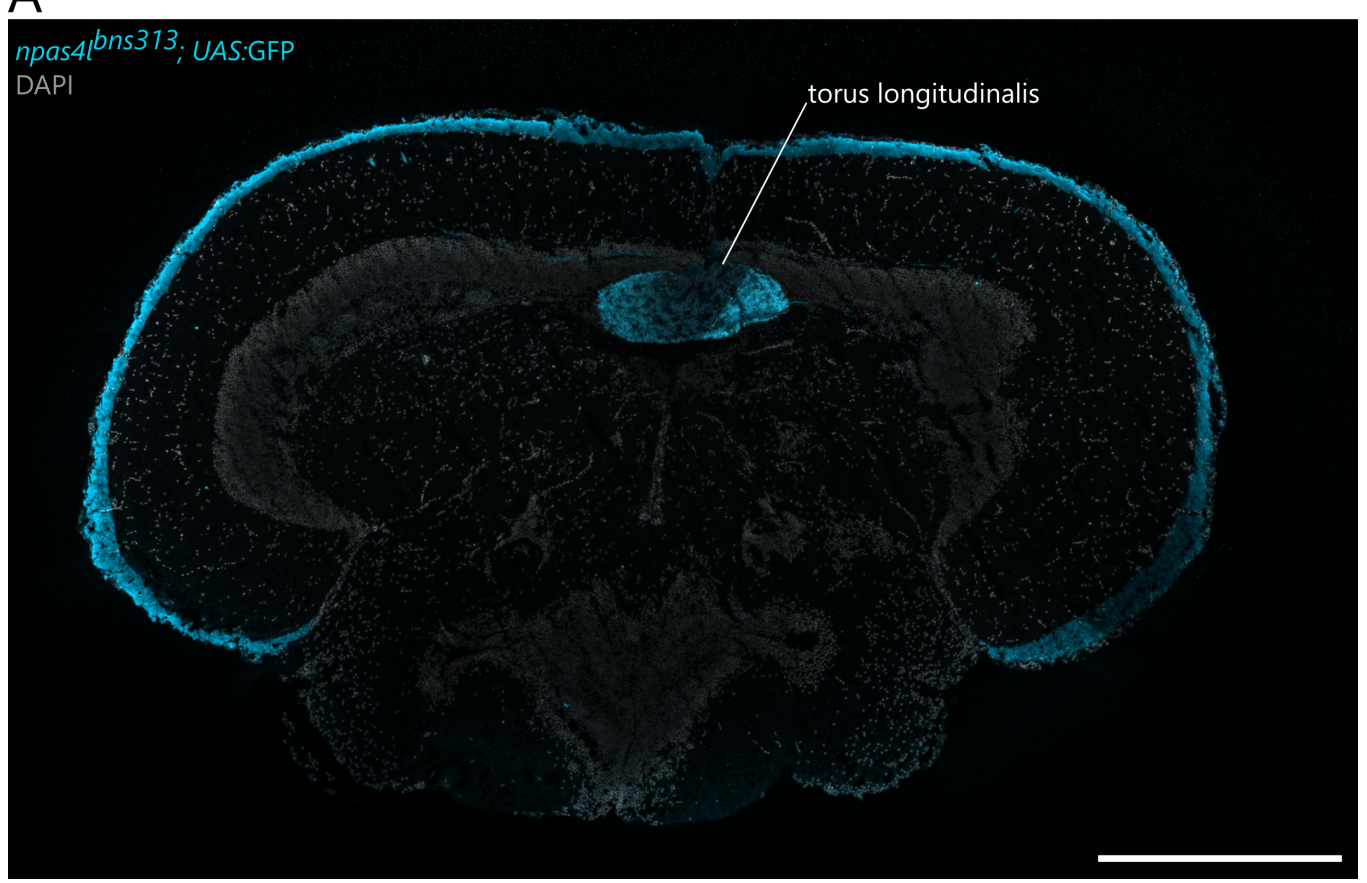

Figure 4.10: Several structures in the adult zebrafish brain express the $n p a s 4 l$ reporter. (A) A strong npas 4 l reporter signal is detected in several brain structures including the optic tectum, the torus longitudinals and the cerebellum. No expression is seen in brain endothelial cells. $\left(\mathrm{A}^{ }\right)$Coronal section at the approximate position indicated by dotted lines shows npas $4 l$ reporter expression in the neuropil layer of the optic tectum and the torus longitudinalis. Brain anatomy was described according to ZFIN ontology aided by the ZebrafishBrain atlas of the midbrain (http://zebrafishucl.org/midbrain). Scalebars: $500 \mu \mathrm{m}$. 
Also, some cells in the adult heart were positive for the npas $4 l$ reporter. To my surprise, these cells were not endothelial cells such as endocardium or coronary endothelium but solitary cardiomyocytes scattered throughout all myocardial layers and structures but especially concentrated around the atrioventricular valve region (Figure 4.11). Co-staining of the section for Caveolin-1 excluded that these cells are epicardial cells. No GFP-positive endothelial cells were detectable in the adult zebrafish.
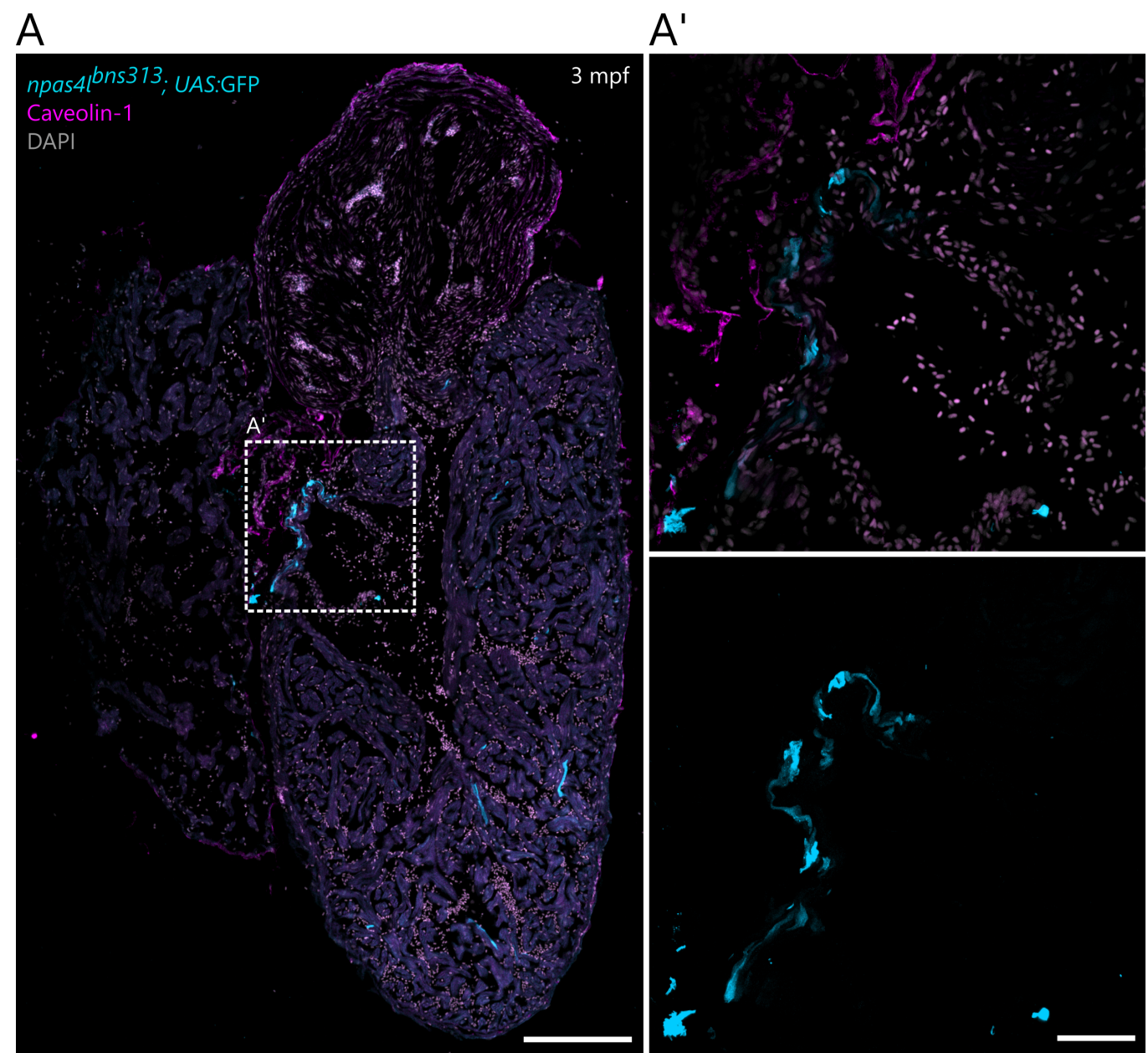

Figure 4.11: npas $4 l$ reporter positive cardiomyocytes are scattered throughout the adult zebrafish heart. (A) Single npas 4 l reporter positive cardiomyocytes are scattered throughout all layers of the adult heart. (A') An accumulation of reporter positive cardiomyocytes is observed around the atrioventricular valves. Caveolin-1 staining excludes these cells to be epicardial cells. Scalebars: $200 \mu \mathrm{m}$ (A), $50 \mu \mathrm{m}$ (A'). I thank Sven Reischauer for his help with the dissection as well as Dr. Anabela Bensimon-Brito and Srinath Ramkumar for the sample preparation including cryo-sectioning. 


\subsection{The npas 4 l reporter is re-expressed dur- ing regenerative processes}

To test whether npas $4 l$ is expressed in the adult vasculature, I decided to analyze the fin vasculature which is especially well suited for high resolution imaging. I amputated and imaged fins of adult reporter positive adults but saw only single npas $4 l$ reporter positive cells in between bone segments. Based on their morphology they may be osteoblasts (Figure 4.12 A-A'). I hypothesized that npas 4 l may not be expressed in differentiated vasculature but might be re-expressed during angiogenesis that occurs during fin regeneration. To test this I collected fins again 3 days post amputation (dpa) and imaged the regenerating part of the fin again.
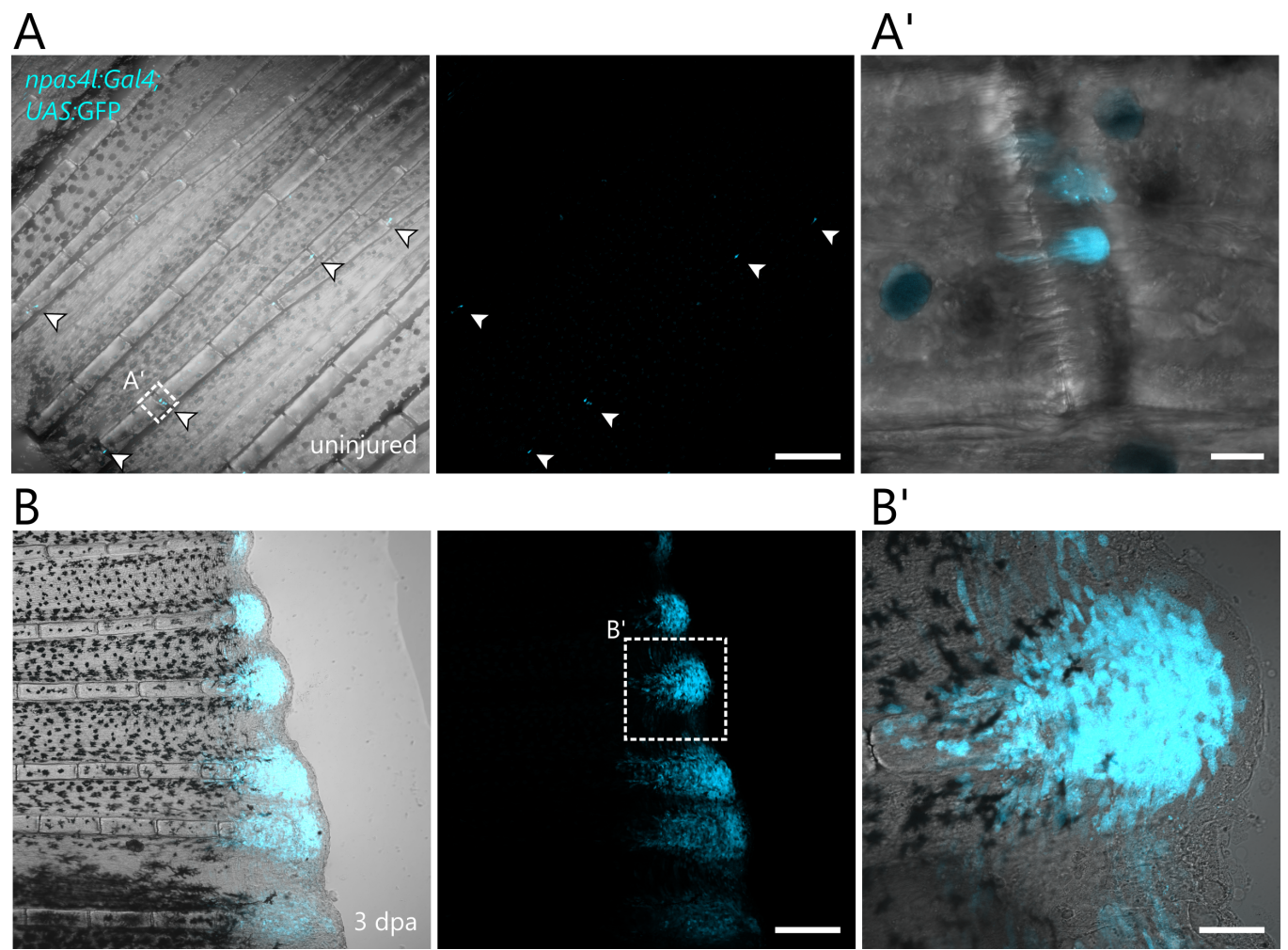

Figure 4.12: The npas $4 l$ reporter is re-expressed during adult fin regeneration. (A) In uninjured fins, the npas 4 l reporter is weakly expressed in solitary single cells in between bone fragments (arrowheads). (A') Based on their morphology, these cells may be osteoblasts. (B) Three days post amputation of the fin, a strong npas 4 l reporter signal was detected in the regenerating part of the fin. (B') npas $4 l$ reporter positive cells do not appear to be endothelial cells but dedifferentiated blastema cells. Scale bars: $200 \mu \mathrm{m}$ (A and B), $10 \mu \mathrm{m}$ (A') $50 \mu \mathrm{m}$ (B'). 
Surprisingly, I did not see expression in regenerating endothelial cells but in the dedifferentiated cell mass of the blastema that forms most of the regenerate. To analyze whether these observations can also be made during embryonic fin-fold regeneration, I amputated the tip of the fin-fold at $48 \mathrm{hpf}$ and imaged the regenerating fin-fold 41 hours after amputation (Figure 4.13) and found a similar upregulation of the npas $4 l$ reporter in the regenerating fin.
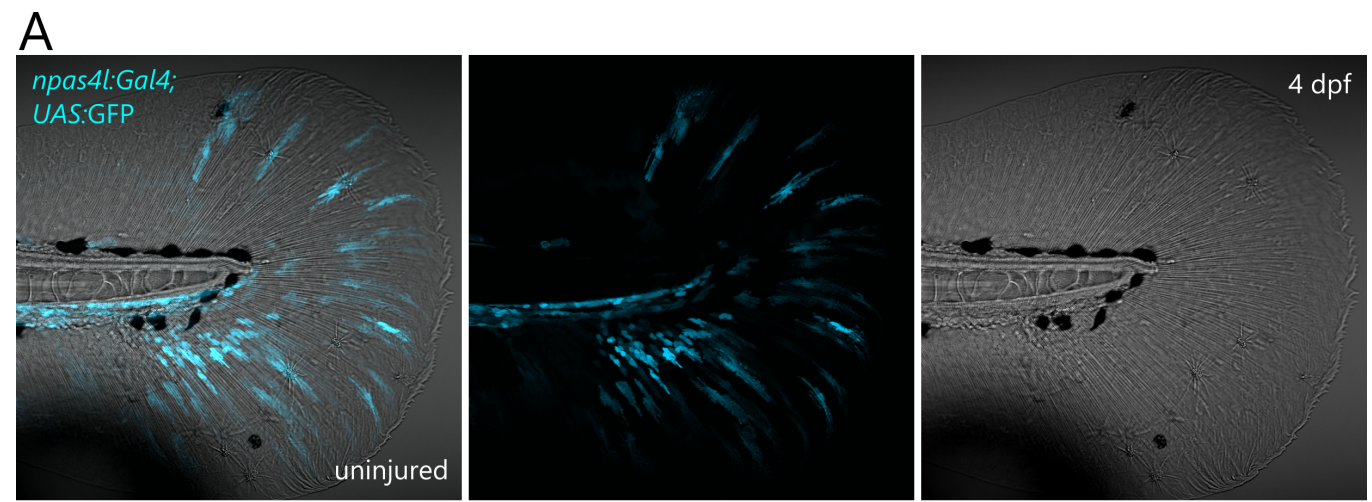

B
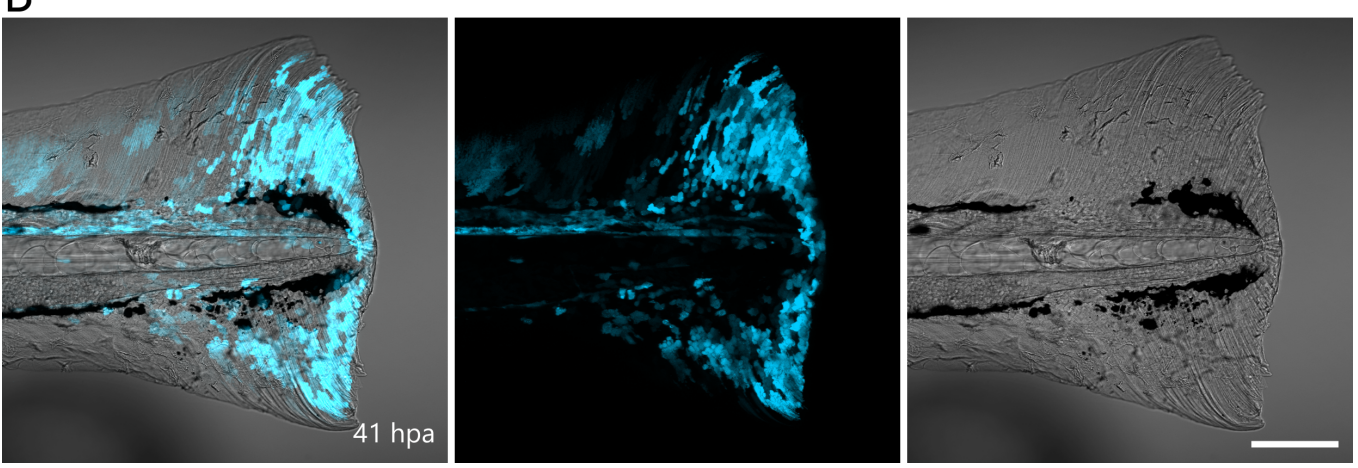

Figure 4.13: The npas 4 l reporter is expressed during embryonic fin-fold regeneration. Strong npas $4 l$ reporter expression can be observed in the regenerating fin 41 hours post amputation (hpa), but not in the uninjured controls. Scale bar: $50 \mu \mathrm{m}$.

The data suggest that npas4l may be upregulated during regenerative processes in the blastema but not in endothelial cells. It will be interesting to investigate whether npas $4 l$ expression only serves as a marker gene of the blastema region or has a physiological role in regeneration process. 


\subsection{Generation of a UAS:mTagBFP reporter line for multi-color imaging of Gal4-re- porters}

Most published fluorescent reporter lines use green or red fluorescent proteins. Reporter lines in other colors often show weak expression or are prone to silencing. Blue fluorescent proteins are rarely used although reporter lines with this color could be combined with both GFP and RFP reporters. One reason is that especially nuclear BFP expression appears to be cytotoxic and lethal during development (unpublished observations). A new version of this protein promises higher chemical stability and brightness but has not been tested in zebrafish. Therefore, I ordered a codon-optimized synthetic nlsmTagBFP2 coding sequence for zebrafish and tested its brightness in vivo by injecting mRNA of this fluorophore. For comparison, I injected mRNA of conventional nls-TagBFP (Figure 4.14).

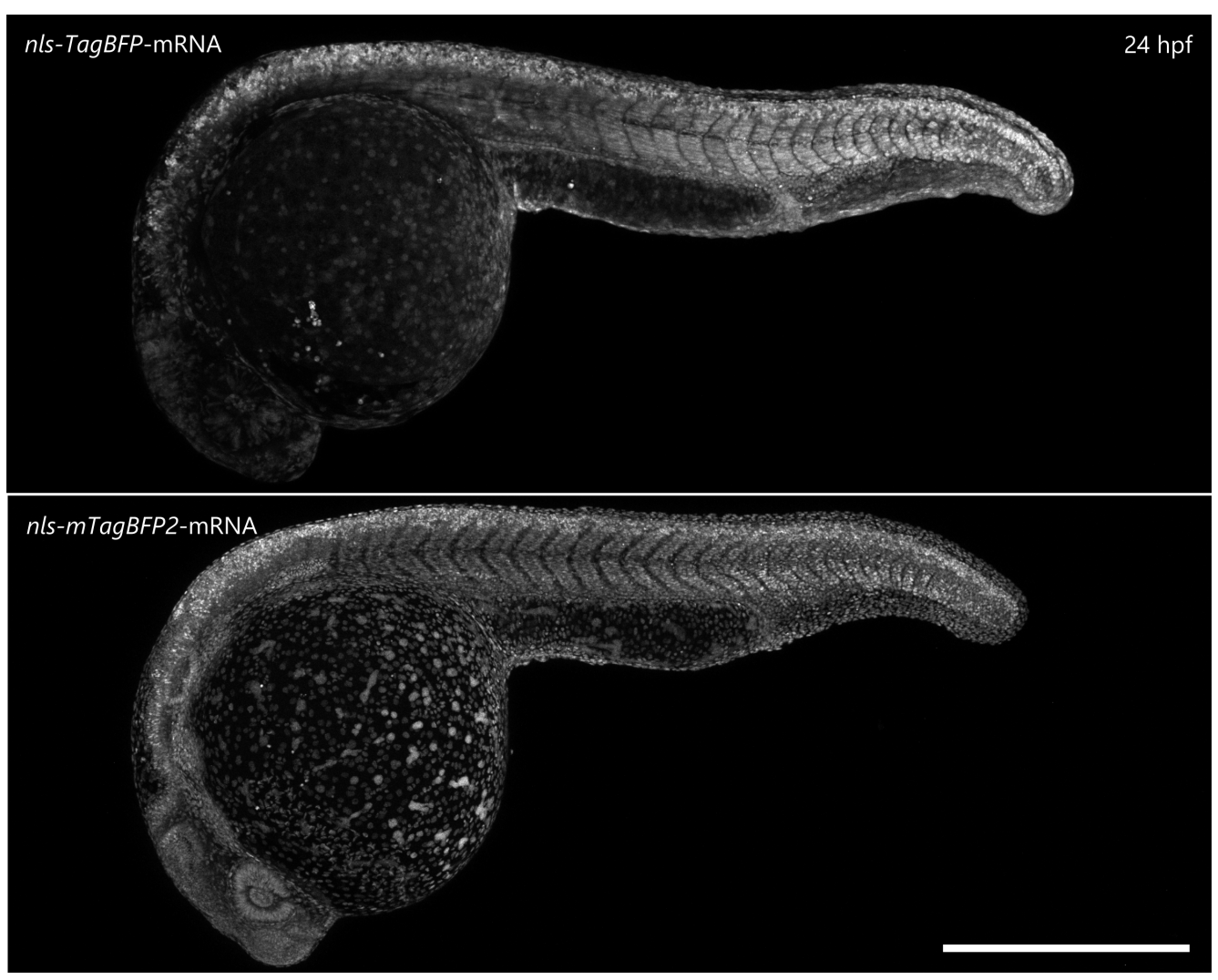

Figure 4.14: Direct comparison of $n l s-T a g B F P$ and $n l s-m T a g B F P$ mRNA expression. mTagBFP2 shows brighter fluorescence and more homogenous distribution of the fluorescent reporter. Scalebar: $500 \mu \mathrm{m}$. 
The results suggest that while TagBFP was more strongly expressed in some tissues, it was patchy and not expressed in nuclei. mTagBFP2 on the other hand showed a ubiquitously strong expression pattern and nuclear loclization. I, therefore, decided to generate a $5 x U A S$ :TagBFP2 reporter line to use this fluorophore with my npas $4 l$ reporter line.

As expected for a short promoter like UAS-elements, founders for this transgenic insertion showed differences in their expression pattern and strength (Table 4.4). I chose to work with founder \#4 as it showed a vascular expression of the fluorescent reporter already at $24 \mathrm{hpf}$ and could be easily detected at 48 hpf (Figure 4.15). Despite an overall weaker fluorescence compared to $U A S$ :GFP reporter lines, this reporter offers an alternative option to visualize Gal4-reporters such as the npas4l reporter in vivo and to use two additional reporters at the same time.

Table 4.4: Screening outcome for the UAS:mTagBFP2 lines. Several different founders were obtained but differed in their expression pattern. Founder \#4 (green) was found to best match the expression pattern of UAS:GFP and was therefore chosen.

\begin{tabular}{l|l} 
Founder ID & Comment \\
\hline female \#1 & yolk and muscles only \\
male \#4 & yolk, expression in vasculature at $24 \mathrm{hpf}$ \\
male \#5 & yolk, heart, muscles \\
male \#13 & yolk, heart, PCV, DA (detected at $48 \mathrm{hpf)}$ \\
male \#14 & yolk, heart, PCV, DA (detected at $48 \mathrm{hpf)}$
\end{tabular}

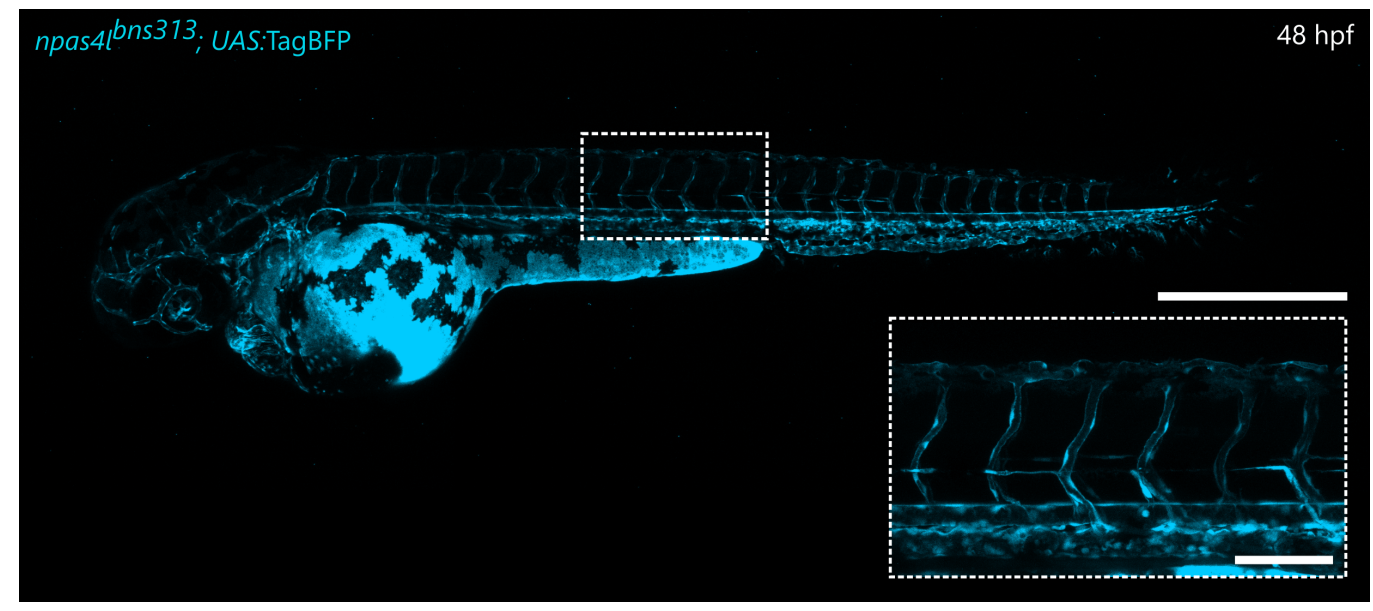

Figure 4.15: The UAS:mTagBFP2 reporter can be used to detect npas $4 l$ reporter expression in the embryonic vasculature at $\mathbf{4 8} \mathrm{hpf}$. Scalebars: $500 \mu \mathrm{m}$ (overview), $100 \mu \mathrm{m}$ (closeup). 


\subsection{Generation of a mutated version of the npas 4 l reporter lacking Npas4l function}

The bns313 allele recapitulated the endogenous expression pattern of npas $4 l$ during embryonic development but was also viable over strong mutant alleles such as the CRISPR allele bns297 (data not shown). Although the reporter was designed to retain the Npas4l function, this characteristic made it impossible to analyze the npas $4 l$ reporter expression in npas $4 l$ mutants with this tool. To solve this problem, I decided to induce an in-frame deletion in the DNA-binding bHLH-domain encoding region in the npas $4 l$ reporter allele that would disrupt the transcriptional activity of Npas4l but retain reporter expression.

I decided to target the beginning and end of exon 2, which encodes most of the DNA-binding domain encoding region. For the beginning of exon2 a published guide RNA design was available (Marass et al., 2019) and for the end of exon 2 new guide RNA's were designed.

Upon injections, reporter positive fish became mosaics, suggesting that the guide RNA's disrupted the coding sequence efficiently leading to a drop in npas 4 l reporter expression. Also, a strong vascular phenotype characterized by the lack of most ISV, a strong pericardial edema, a malformed heart and ectopic npas $4 l$ reporter expression in skeletal muscle cells were observed in most injected fish (Figure 4.16).

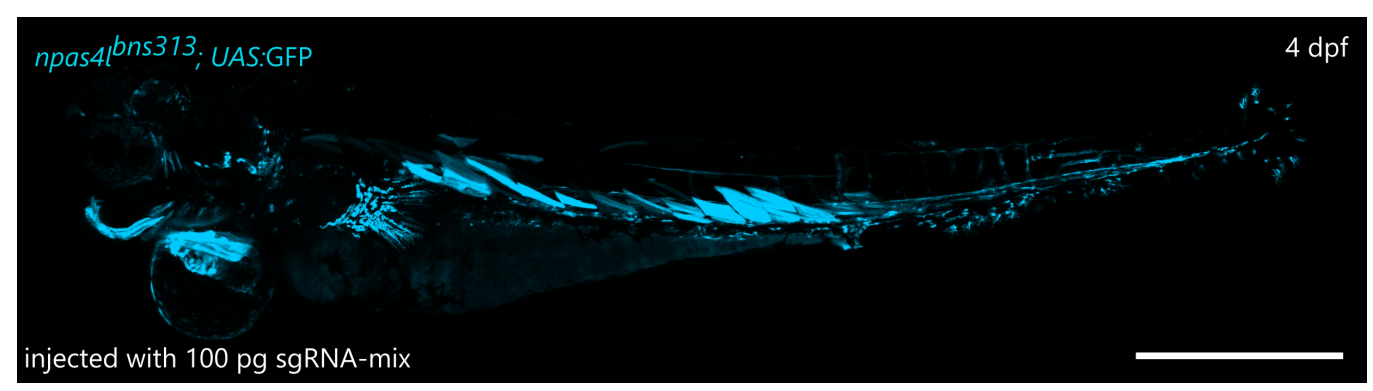

Figure 4.16: Targeting the npas $4 l$ coding sequence in the $n p a s 4 l$ reporter with CRISPR/Cas9 causes a strong vascular phenotype. Validation of the strategy for mutating the npas $4 l$ reporter to convert it to a non-functional allele. Injected P0 embryos showed pericardial edema and a loss of endothelium similar to the npas $4 l$ mutant phenotype. An increase in skeletal muscle cells was observed as well. Scalebar: $500 \mu \mathrm{m}$.

As the phenotype caused by the targeting reagents looked both very strong and similar to the expected cloche/npas 4 l mutant phenotype, it appeared to 
be an on-target effect. These early results were promising but made raising the P0 animals and screening for the allele problematic due to the high lethality. I followed two different strategies to optimize the chances of obtaining founders:

1. a decreased dose of npas $4 l$ targeting reagents to decrease the efficiency and phenotype

2. the use of a germline targeted cas9-nanos3'UTR mRNA

While only few embryos survived even low doses of the guideRNA mix with conventional cas9-mRNA, embryos injected with cas9-nanos3'UTR mRNA looked almost completely normal and did not show defects. These observations validated the germline targeting of Cas9 as a promising approach, but as there are only very few germline cells in the embryo and larvae, these injections had to be done without verification of cutting efficiency by HRMA. Therefore, embryos injected with both strategies were raised for screening.

During the founder screening, I had to ensure that the indel was present on the same allele that carried the reporter and that the reporter was still expressed. Therefore, I screened by outcrossing adult P0 fish and testing only reporter positive embryos by PCR spanning the entire second exon. Small deletions were not detected by this strategy, but the aim was to generate a large deletion of the DNA-binding site. A single PCR-band would indicate that both the P0 as well as the wild-type fish crossed to it carry a band without deletions, whereas a double band would indicate the presence of an indel on one of the two alleles. As the embryos were all reporter positive offspring of outcrossed fish, this shifted band had to be transmitted on the same allele that carried the reporter allele and not disturb the reporter expression. Otherwise, fluorescence would not have been observed.

Table 4.5: Screening outcome for mutating the DNA-binding domain encoding region of the npas 4 l Gal4-reporter allele. Several out-of-frame alleles and one inframe allele were found. The in-frame allele transmitted by male \#24 (green) was used in subsequent experiments. The column phenotype describes whether the allele failed to complement the mutant showing a cloche-like phenotype.

\begin{tabular}{l|l|l} 
Founder \# & Allele(s) & Phenotype \\
\hline male \#7 & $-101 \mathrm{bp} /$ n.a. & yes \\
male \#10 & $-29 \mathrm{bp}$ & n.a. \\
male \#17 & n.a. & n.a. \\
female \#21 & $+17 \mathrm{bp}$ & n.a. \\
female \#22 & $-5 \mathrm{bp}$ & yes \\
male \#24 & $+36 \mathrm{bp} /+59 \mathrm{bp}$ & yes/yes
\end{tabular}


Although many P0 fish showed a decreased number or absence of fluorescent offspring indicating a deletion that silenced the reporter expression (observations not quantified and data not shown), I found a surprising number of embryos that had out-of-frame indels (screening summarized in table 4.5) and retained reporter expression through complex alternative splicing as determined by sequencing of cDNA bands (data not shown). However, I found one $+36 \mathrm{bp}$ indel in the 3 '-end of exon 2 (Figure 4.17A), that seemed to disrupt the DNA-binding site encoding region in the bHLH domain (Figure 4.17B) but did not interfere with the predicted splice sites of exon 2. PCR on genes in the telomeric region distal to npas 4 l suggested that no large off-target deletions had occurred (Figure 4.17B). Sequencing PCR products spanning the $\mathrm{cDNA}$ sequence from exon 1 to the reporter insertion,

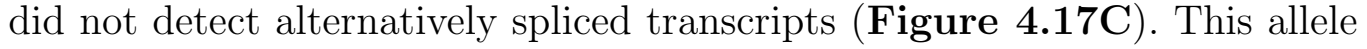
is henceforth called the mutated npas4l reporter allele bns423.

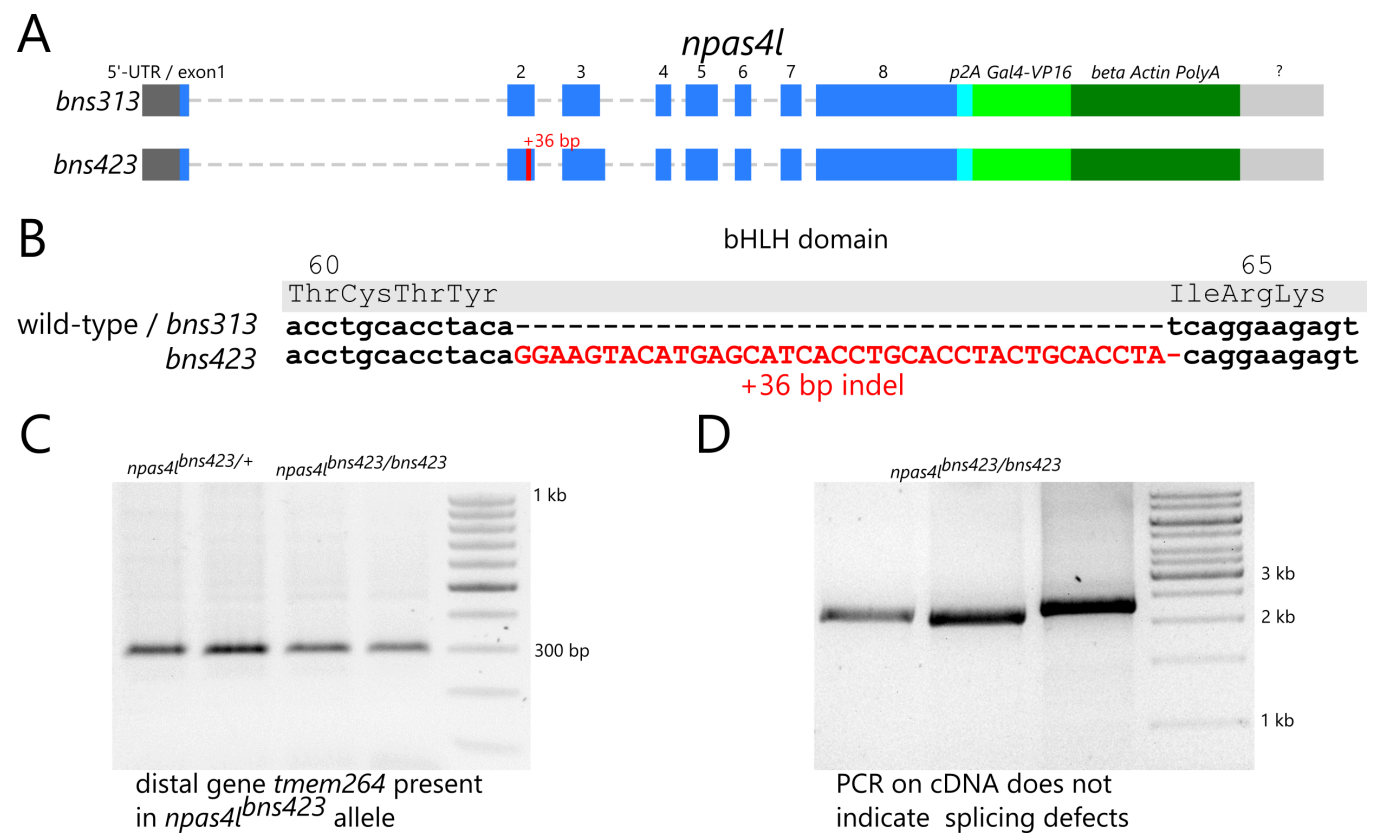

Figure 4.17: Generation of a npas 4 l reporter variant carrying a $+36 \mathrm{bp}$ indel in exon 2. (A) Insertion site of -p2A-Gal4-VP16 in exon 8 of the endogenous npas 4 l locus. +36 bp indel in exon 2 of the bns423 allele highlighted in red. (B) Partial sequence of the npas $4 l$ exon 2, which encodes the second helix of the DNA-binding domain, showing the $36 \mathrm{bp}$ indel (highlighted in red) in the bns423 allele. (C) Presence of the distal gene tmem264 in homozygous npas $4 l^{\text {bns } 423}$ mutants as tested by PCR on genomic DNA. (D) PCR on cDNA of homozygous npas $4 b^{\text {bns } 423}$ mutants spanning all intron-exon-boundaries shows a clear single band with three different primer pairs indicating no detectable splicing defects of the mutated allele. 


\subsection{Characterization of the mutated npas $4 l^{\text {bns } 423}$ reporter allele}

Next, I tested whether the newly found mutated reporter allele showed a npas $4 \mathrm{l}$ mutant phenotype. To this end, I crossed the allele to carriers of the strong npas4l mutant allele bns297. The bns423 allele failed to complement the bns297 mutant allele (Figure 4.18). In the pan-vascular fli1a reporter background, trans-heterozygous npas $4 l^{\text {bns } 423 / b n s 297}$ fish were phenotypically indistinguishable from other strong homozygous mutant alleles of npas $4 l$ such as the s5, m39 and bns297 alleles, characterized by a complete absence of fli1a reporter positive endocardium and most endothelial and blood cells (Figure 4.18). Mutant embryos died before 5 dpf (data not shown). The reporter was expressed in similar levels in both heterozygous and mutant
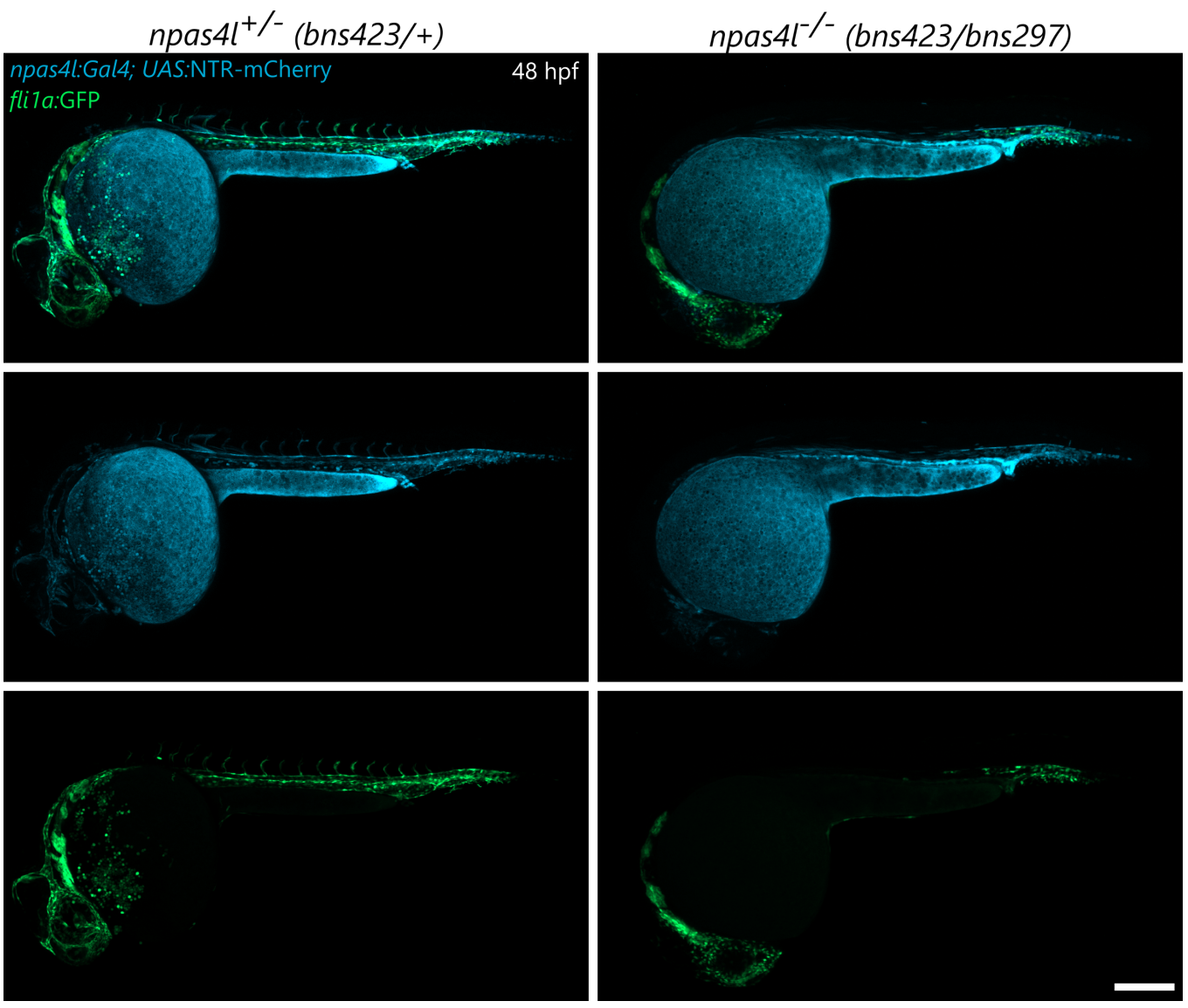

Figure 4.18: The $n p a s 4 l^{b n s 423}$ allele fails to complement the $n p a s 4 l^{b n s 297}$ allele. (A) npas $4 l^{\text {bns } 423 / b n s 297}$ trans heterozygous mutant embryos display a severe reduction in fli1 a positive endothelial cells characteristic of cloche/npas $4 \mathrm{l}$ mutants. Scale bars: $200 \mathrm{\mu m}$. 
embryos, although the expression pattern of the reporter changed drastically in the mutants.

I decided to compare homozygous carriers of the wild-type like bns313 reporter allele and of the mutated bns423 allele side by side (Figure 4.19). While in homozygous bns313 carriers, reporter expression is mainly observed in endothelial cells. This signal is lost in homozygous bns423 carriers. Instead, skeletal muscle cells and a bilateral population of rounded cells on the ventral side of the embryo could be observed (Figure 4.19B, Figure 4.20A and B). Both the muscle and ventrolateral round cell populations were stable and could still be detected at $90 \mathrm{hpf}$ (Figure $\mathbf{4 . 2 0 C}$ ). In addition, the mutant hearts showed the typical bell shape that is characteristic

A

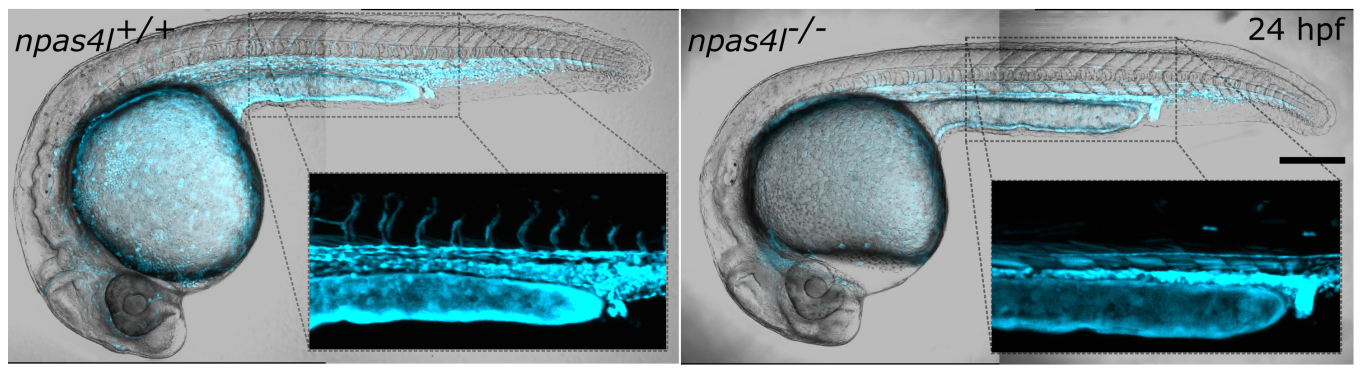

B

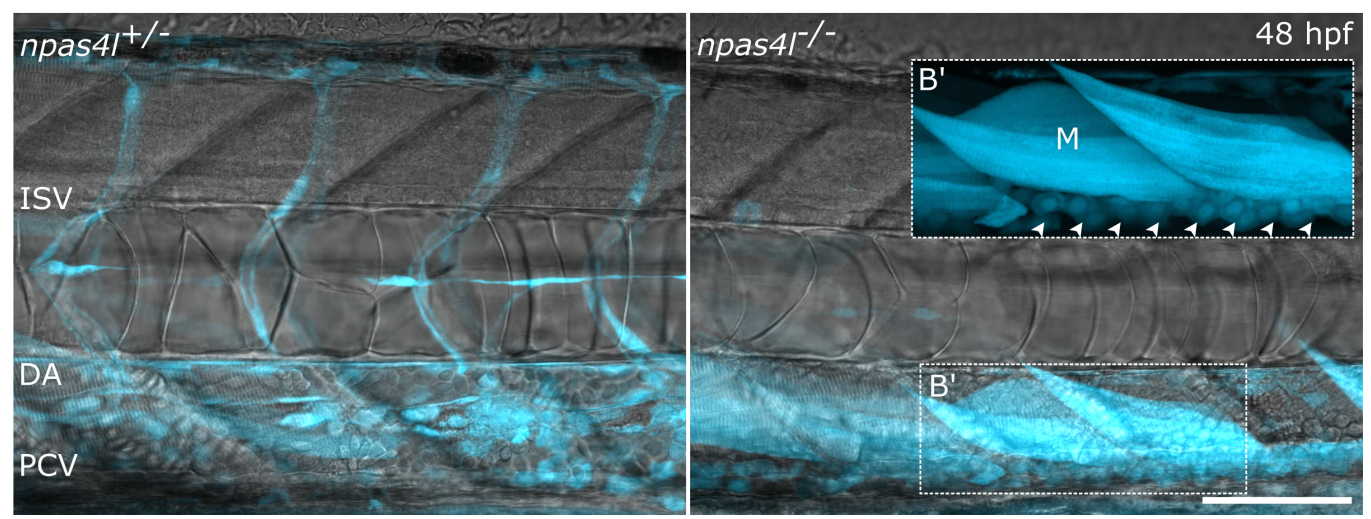

Figure 4.19: Phenotypic comparison between the wild-type like bns313 and the mutated bns423 alleles. (A) Incrosses of both lines with UAS:GFP show the lack of ISVs in homozygous npas $4 l^{\text {bns } 423 / b n s 423}$ mutants (right), but not in homozygous npas41 ${ }^{\text {bns313/bns313 }}$ reporter embryos (left). (B-B') npas $4 l$ reporter expression in the vasculature of npas $4 l$ heterozygous (left) and homozygous mutant (right) embryos. npas $4 l$ reporter expression marks endothelial cells in heterozygous embryos but skeletal muscle (M) and a ventral row of rounded cells in mutants (arrowheads). ISV: intersomitic vessel, DA: dorsal aorta, PCV: posterior cardinal vein. Scale bars: $200 \mu \mathrm{m}(\mathrm{A}), 50 \mu \mathrm{m}(\mathrm{B})$. 
for cloche mutants (Figure 4.21A) and optical sections through these hearts confirmed that only a single layer of cells was present, suggesting an absence of endocardial cells (Figure 4.21A').

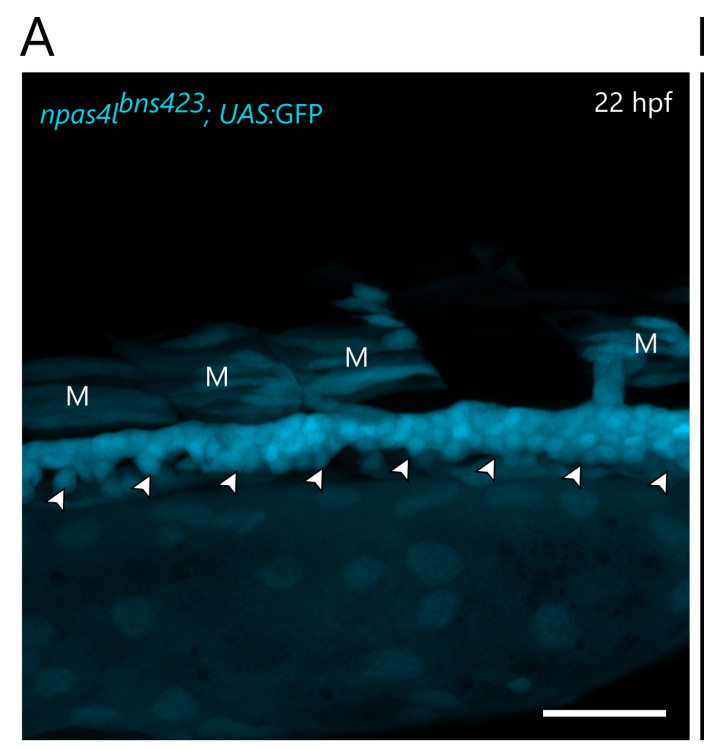

\section{B}
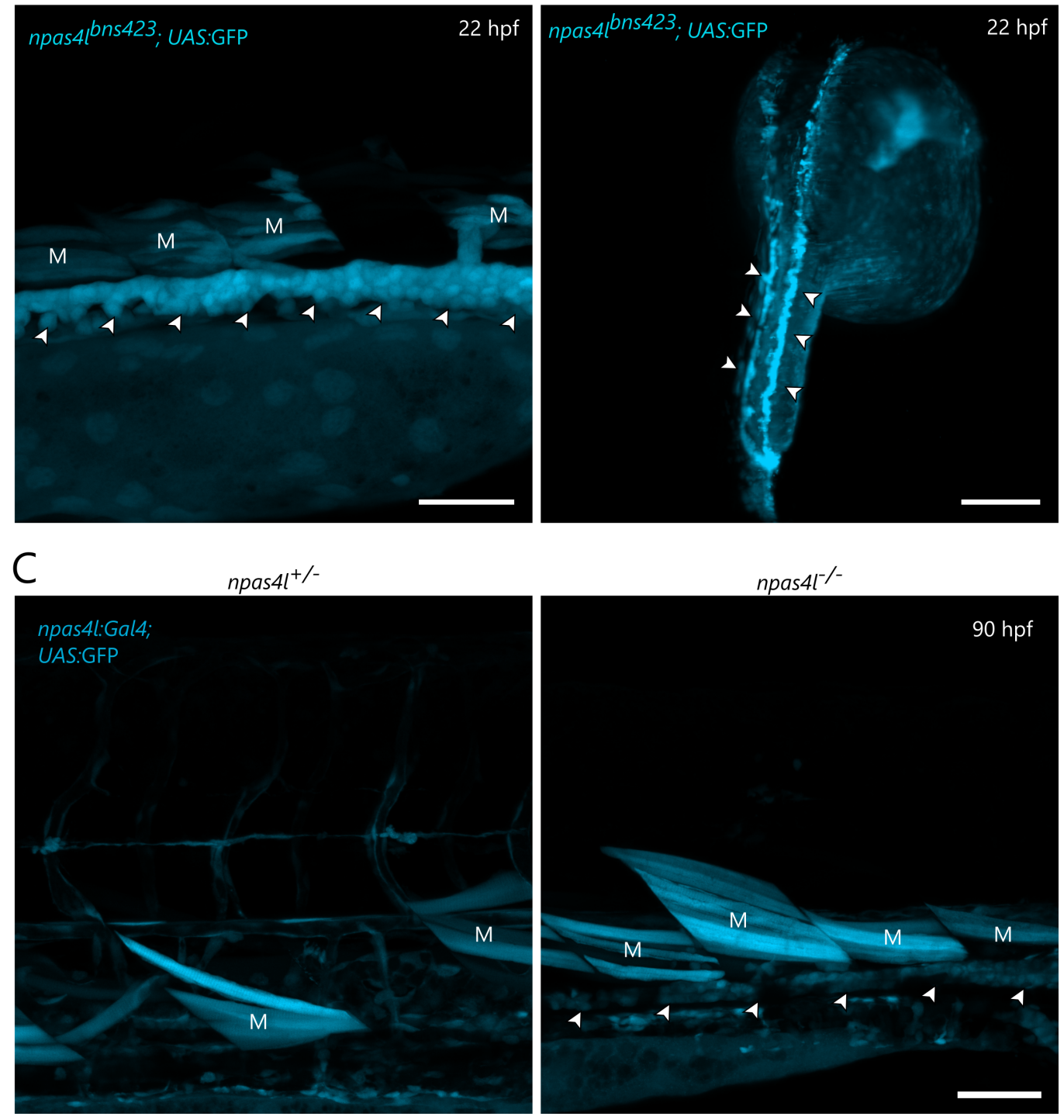

Figure 4.20: Ventrolateral cells are present in the npas $4 l$ mutant and form a stable cell population. (A-B) A bilateral population of ventral cells in the trunk can be detected in the embryos (arrowheads), indicating a previously undescribed phenotype in npas $4 l$ mutants. Also, an increased skeletal muscle population $(\mathrm{M})$ can be detected. (C) npas 4 l reporter expression in $n$ pas $4 l^{+/-}$and npas $4 l^{-/-}$embryos at $90 \mathrm{hpf}$. The ectopic skeletal muscle expression (M) and expression in ventrolateral cells (arrowheads) is still visible at this stage. Scalebar: $50 \mu \mathrm{m}(\mathrm{A}, \mathrm{C}), 200 \mu \mathrm{m}$ (B). 

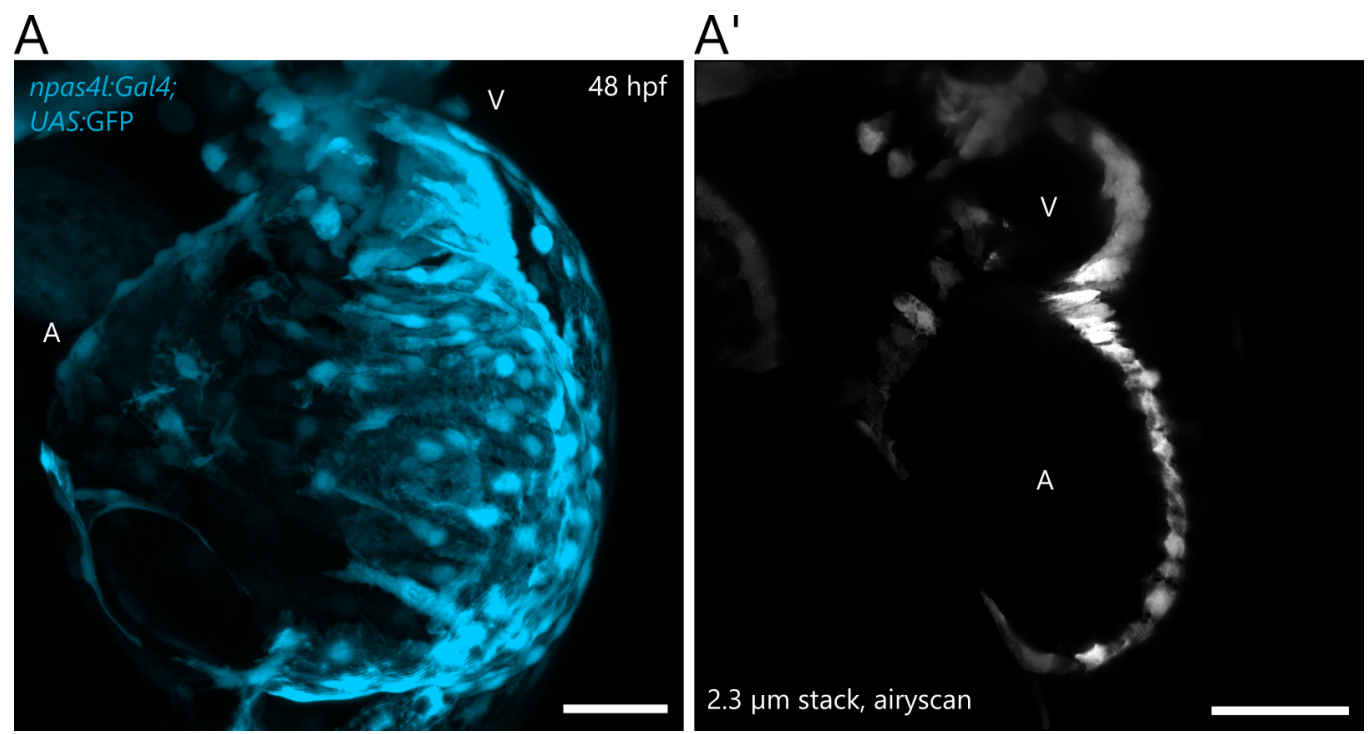

Figure 4.21: The mutation in the $n p a s 4 l$ reporter allele causes a characteristic npas $4 l$ mutant heart phenotype. (A) Characteristic bell shaped heart of the npas $4 l$ mutants. (A') A $2.3 \mu \mathrm{m}$ optical section of the same heart shows only a cardiomyocyte layer but no endocardial cells. A: atrium, V: ventricle. Scalebars: $50 \mu \mathrm{m}$.

A contribution of npas $4 l$ positive cells to the skeletal muscle lineage in npas $4 l$ mutants was an interesting finding but seemed plausible as a similar phenotype had been observed for etsrp positive cells in etsrp mutants. As etsrp is a direct transcriptional target of Npas4l and npas $4 l$ mutants lack etsrp expression, this phenotype appeared to be plausible. However, rounded ventrolateral cells in the cloche mutant or related mutants had never been reported before. I wondered what exactly these cells were, hypothesizing that they might be angioblast precursors that failed to migrate towards the midline. To test this hypothesis, I performed cross-sections to assess the anatomical location of the ventrolateral npas $4 l$ reporter positive cells and followed these cells through their migration (Figure 4.22).

Surprisingly, the location of the npas 4 l reporter positive cells in npas 4 l mutants suggested that these cells were pronephric tubule cells. Immunohistochemistry for the early intermediate mesoderm marker Pax2a confirmed a co-localization of this marker and the npas $4 l$ reporter in many pronephric tubule cells (Figure 4.22B). Imaging throughout the angioblast migration phase showed that at $10.7 \mathrm{hpf}$, mutant and heterozygous npas $4 \mathrm{l}$ reporter positive cells were located lateral to the somites. While npas $4 \mathrm{l}$ reporter positive cells in heterozygous animals migrated towards the midline and coalesced into a vascular cord in later stages, npas $4 l$ reporter positive cells in the mutants 
remained lateral (Figure $4.22 \mathrm{C}$ ).

A

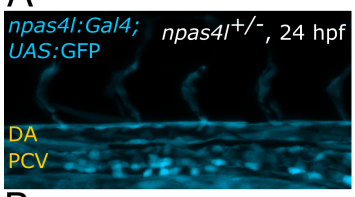

B
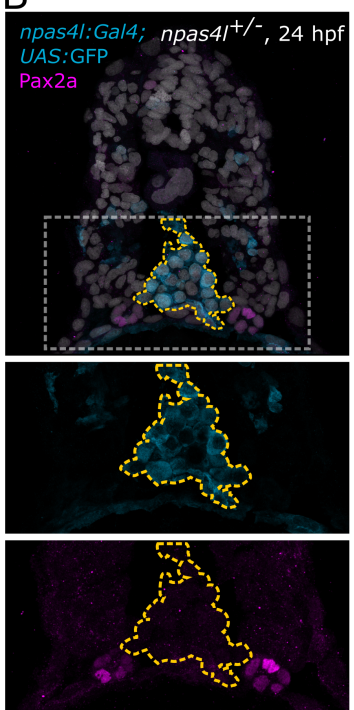
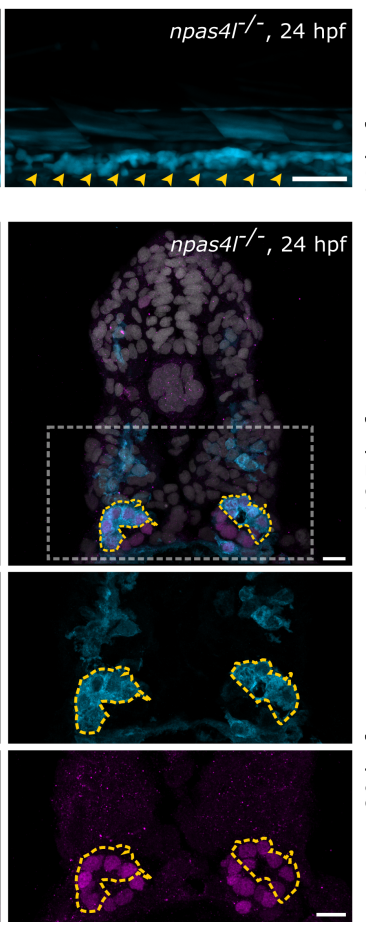
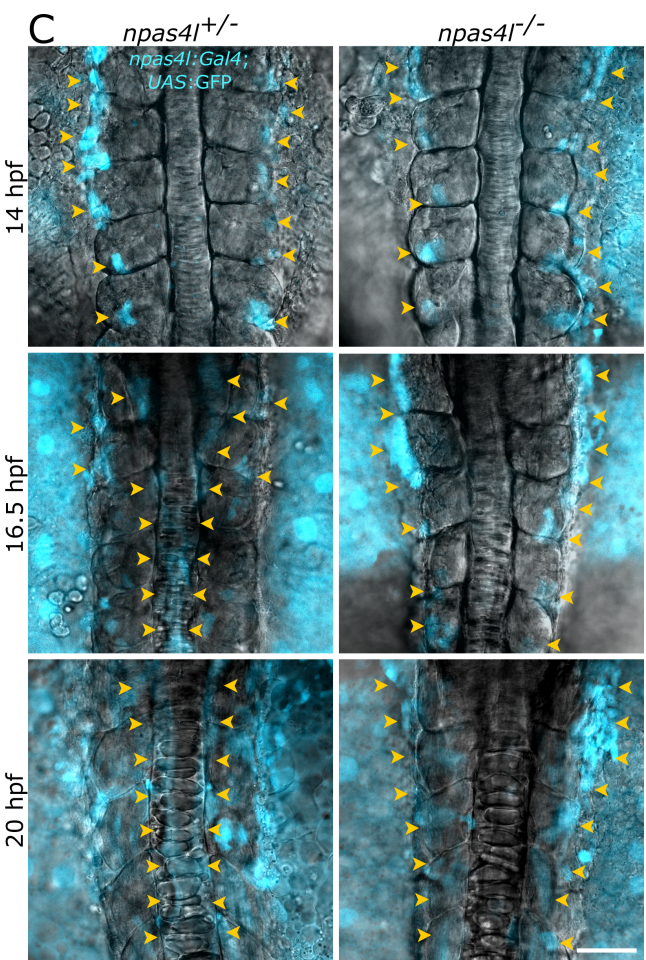

Figure 4.22: Endothelial progenitors in npas 4 l mutants fail to migrate to the midline. (A) npas $4 \mathrm{l}$ reporter expression in ventral cells of $24 \mathrm{hpf} n p a s 4 \mathrm{l}^{+/-}$(left) and npas $4 l^{-/}$(right) embryos. The main axial vessels (DA and PCV) are visible in npas $4 l^{+/-}$ embryos, while only cells located more ventrally are detected in npas $44^{/-}$embryos (arrowheads). (B) Transverse sections of $20 \mathrm{hpf} n$ pas $_{4} \mathrm{l}^{+/-}$(left) and npas $4 \mathrm{l}^{-/-}$(right) embryos. The endothelial progenitors (outlined by yellow dotted lines) in npas\&l mutants fail to reach the midline unlike those in wild-type siblings; they also remain round. Ventrolateral npas $4 l$ reporter positive cells in npas $4 l^{-}$embryos are also Pax2a + and are part of the pronephric tubules. (C) Dorsal views of $14,16.5$ and $20 \mathrm{hpf}$ npas $4 \mathrm{l}^{+/-}$(left) and npas $4 \mathrm{l}^{-/}$ (right) embryos. Arrowheads point to npas 4 l reporter positive endothelial progenitors. DA: dorsal aorta, PCV: posterior cardinal vein. Scale bars: $50 \mu \mathrm{m}(\mathrm{A}, \mathrm{C}), 10 \mu \mathrm{m}(\mathrm{B})$.

In summary, the data confirmed the mutated reporter allele bns423 to be a strong npas $4 \mathrm{l}$ mutant allele that preserved the expression of the reporter. While this reporter looked like the wild-type like bns313 reporter allele in heterozygous animals, the expression pattern changed drastically in homozy-

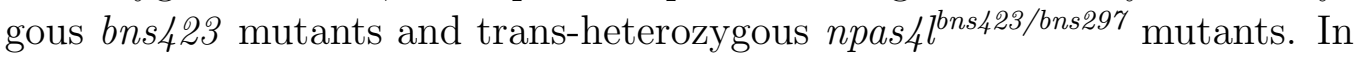
addition to all the known phenotypes of cloche/npas $4 \mathrm{l}$ mutants, the reporter indicated the presence of npas 4 l reporter positive skeletal muscle cells and ventrolateral rounded cells that turned out to be Pax2a positive pronephric tubule cells. 


\subsection{Bulk transcriptomic profiling of the $n p a s 4 l$ reporter positive lineage in npas $4 l$ het- erozygous and mutant embryos}

To describe the changes molecularly that we saw on the phenotypic level and to search for other phenotypes caused by the lack of Npas4l function that were not immediately evident by fluorescence, I profiled the npas $4 l$ reporter positive lineage in npas $4 l$ heterozygous and mutant embryos.

As the npas $4 l$ reporter was expressed in several different cell types, which could complicate the analysis, I decided to restrict the profiling to the tail of the embryo by amputating and dissociating it at $20 \mathrm{hpf}$ prior to fluorescent activated cell sorting (FACS) for npas $4 \mathrm{l}$ reporter positive cells and fli1a reporter positive cells. The timepoint $20 \mathrm{hpf}$ was chosen according to technical considerations as the cloche/npas 4 l mutant phenotype in the reporter allele becomes evident clearly at around $17 \mathrm{hpf}$ providing a window of around 3 hours for sorting and sample preparation. Cells from heterozygous and mutant embryos were then compared by bulk exome sequencing (Figure 4.23 ).

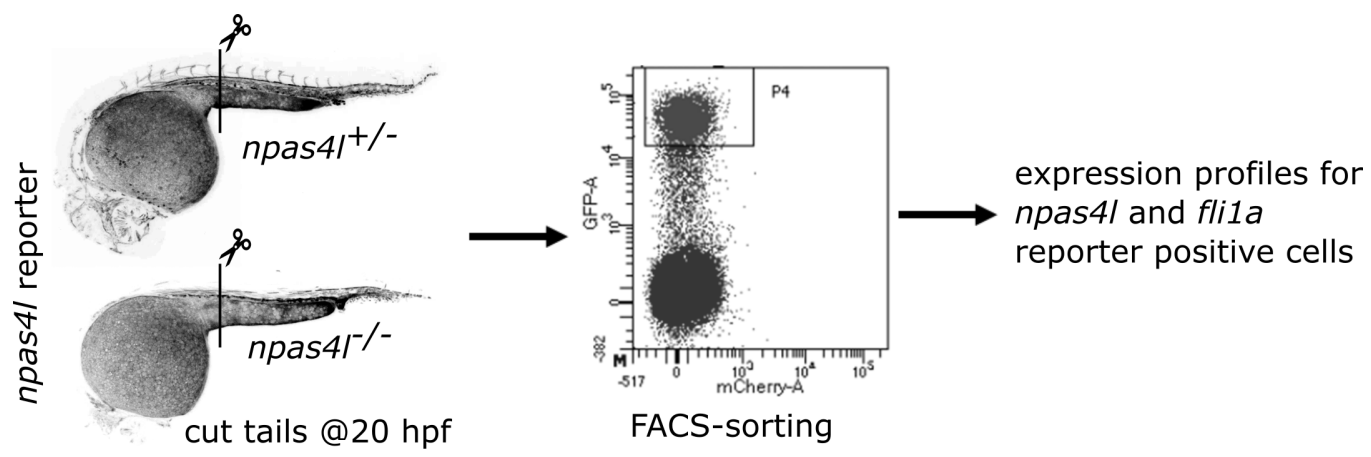

Figure 4.23: Expression profiling workflow. Tails of npas4l reporter positive and fli1a reporter positive embryos were cut, their cells dissociated and sorted using FACS for the GFP expression of the reporter. Expression profiles of npas $4 l$ and fli1a reporter positive cells were profiled in bulk. A single cell RNAseq of npas $4 l$ reporter positive cells was added later to increase the resolution in the dataset. Thanks to Ann Atzberger and Kikhi Khrivono for their help with FACS sorting and to Stefan Günther at the Bioinformatics core facility for the library preps, sequencing and basic analyses. 
A

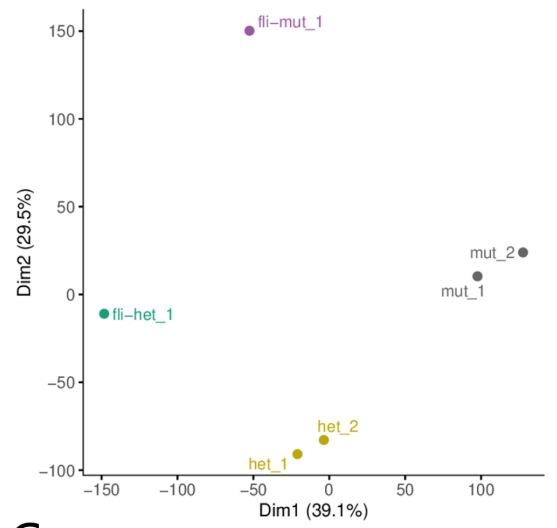

B

Volcano Plot

regulation: counts $>=5, \log 2 \mathrm{fc}<>+-0.585, \mathrm{FDR}<=0.05$

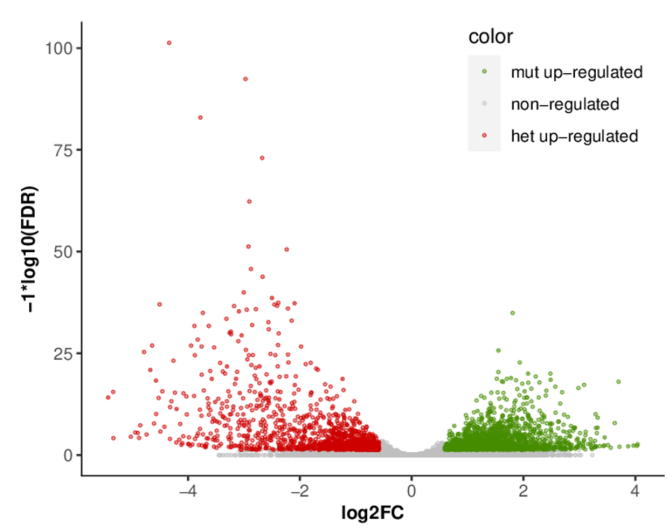

C
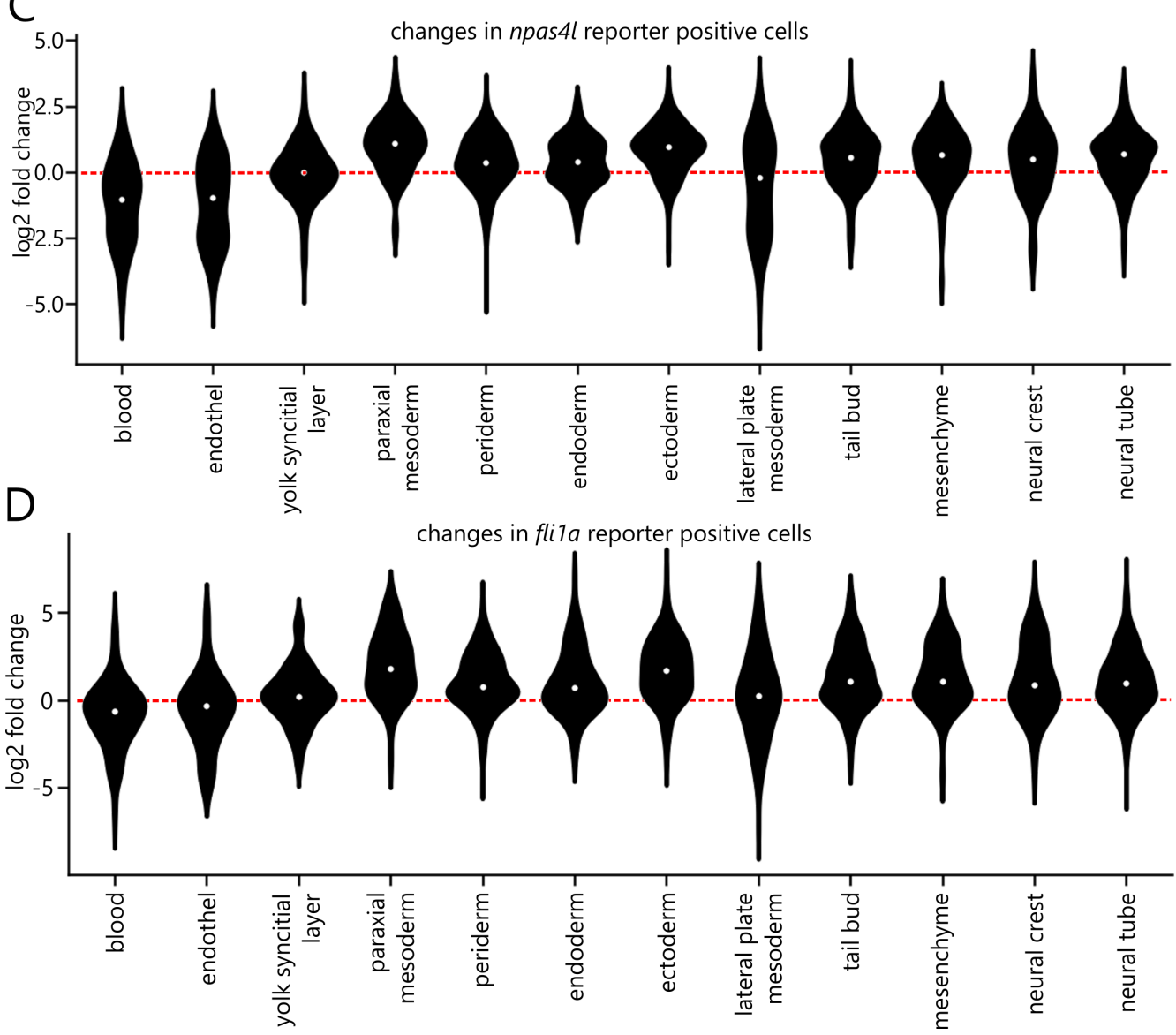

Figure 4.24: Strong global changes can be detected in the bulk exome sequencing data. Figure legend continued on the next page. 
Figure 4.24: (A) Dimensional reduction of the two gene groups that drive most differences between the samples plotted as principal component analysis (PCA). DESeq normalized gene counts were log-transformed and genes with counts lower than 5 removed to reduce the impact of genes with low counts. Two biological replicates for npas $4 l$ reporter positive (het and mut) cells and one biological replicate for filia reporter positive cells (fli1-het and fli-mut) were analyzed. Biological replicates cluster together well. (B) Volcano plot showing $\log 2$ fold change of all detected genes in mutants compared to heterozygous controls on the x-axis and false discovery rate (FDR) on the y-axis $(-\log 10$ transformed). Genes with at least 5 reads, FDR $\leq 0.05$ and a log2-fold change $>0.585$ (green) or $<-0.585$ (red) have been highlighted. 2710 genes were significantly regulated by these criteria between heterozygous and mutant npas $4 l$ reporter positive cells. For fli1a positive cells this analysis was not performed as only one replicate was sequenced for each condition. (C-D) log2-fold changes of genes associated with different embryonic structures as determined by search against the ZFIN in situ expression database. (C) and fiila (D) reporter positive cells were plotted in a violin plot. Median indicated as white dot; red dotted line highlights the $\log 2$ fold change of 0 . A positive $\log 2$ fold change indicates higher expression in the mutant. Deregulation of genes was seen in all sampled structures. The PCA plot and Volcano plot shown in panel (A) and (B) were part of the basic analyses performed by Stefan Günther from the Bioinformatics core facility. The scripts for matching log2-fold changes to various structures were self-written but based on the $\log 2$-fold changes provided by Stefan Günther.

The fli1a sorting was mainly intended to filter which of the genes detected in the profile were expressed in heterozygous or the few endothelial cells remaining in the cloche mutants. Therefore, only one biological replicate of fli1a positive cells in npas 4 l heterozygous and mutant animals was sequenced whereas two biological replicates were sequenced for npas $4 l$ reporter positive cells.

Principal component analysis suggested a close clustering of the biological replicates and the main contrast to be lying between individual conditions (Figure $4.24 \mathrm{~A}$ ). As expected, fli1a reporter positive cells and npas 4 l reporter positive cells were transcriptionally different to one another, even in heterozygous fish as the npas $4 l$ reporter does not only label endothelial cells at this stage. Volcano plots showed, however, that 2710 genes - more than $12 \%$ of all genes detected in this dataset - were significantly regulated (Figure 4.24B). Regulated GO-terms were broad and included an up-regulation of genes involved in proliferation, a cadherin switch from $c d h 5$ to other cadherins including $c d h 2$ as well as an up-regulation of Wnt pathway related genes such as wnt5b and of many mesenchymal genes (data not shown). 
This change reflected the mixture of different alternative cell fates in the mutants but made the interpretation challenging, especially via candidate approaches. Therefore, I wrote a short script searching the ZFIN database for genes that are expressed in various tissues or cell types of choice based on published in situ hybridization data. The log2-fold changes of these genes detected in the dataset were then displayed as violin plots indicating the shift of expression in genes associated with certain anatomical structures. Surprisingly, genes expressed in most of these structures were highly regulated and variable including various mesodermal as well as endodermal and ectodermal genes (Figure $\mathbf{4 . 2 4 C}$ ). I repeated this analysis for fli1a positive cells remaining in the tail region of npas $4 l$ mutants and came up with a similar picture. Although the mutant cells showed expression of most early vascular markers such as cdh5, etsrp and fli1a, blood-related genes such as tal1, lmo2 and gata1a were not expressed by these cells suggesting a lack of hemogenic potential in the mutant endothelium. Genes that are usually enriched in arterial endothelial cells like $f t 1, k d r l$, egfl7 and $d l l 4$ or in venous endothelium like flt4 and lyve $1 b$ all had a downwards trend suggesting that the remaining endothelial cells in npas4l mutants were unlikely to be predominantly arterial or venous cells. Instead, they appear to exhibit either a defective arterio-venous specification in the absence of Npas4l or a delayed differentiation (data not shown).

In summary, the experiment suggested very strong global transcriptional changes indicating several lineage specification or restriction defects. The problem was the severity of changes between the two conditions and the number of genes involved. To address these challenges, I profiled these cells again using single cell RNAseq.

\subsection{Single cell transcriptomic profiling of the npas 4l positive lineage in npas 4 l het- erozygous and mutant embryos}

I decided to sequence 5555 npas 4 l reporter positive cells from npas 4 l heterozygous and mutant embryos. Although I considered using cells from the entire fish, I could not estimate how many distinct cell populations and rare cell types to expect given the strong changes detected in the bulk RNAseq profiles. Therefore, followed the same strategy described in figure $\mathbf{4 . 2 2}$, in which tails from $20 \mathrm{hpf}$ heterozygous and mutant embryos were cut and sorted for fluorescence. 
As expected from the bulk RNAseq experiment, the initial UMAP representations showed dramatic changes between the two genotypes (Figure 4.25A). Clustering into 8 groups reflected the data best (Figure 4.25B). For the cluster annotation, I re-used the scripts I had written to analyze the bulk RNAseq by searching marker genes for each cluster against the ZFIN in situ database and looking up where these genes are usually expressed. I did the same analysis using the annotations of a published zebrafish scRNAseq atlas (Wagner et al., 2018) and curated the annotations manually. Five of the top markers used to identify and annotate each cluster are plotted in Figure $4.25 \mathrm{~F}$.

The cluster annotations showed that in heterozygous embryos most npas $4 \mathrm{l}$ reporter positive cells are endothelial and blood cells, while these clusters are almost completely absent in the mutant embryos. These changes reflect the known loss of endothelium and blood in npas 4 l mutants. Interestingly, the data also showed a clear increase of paraxial mesoderm-derived muscle cells and intermediate mesoderm-derived pronephric tubule cells. Together, these changes reflected the majority of changes observed between the two genotypes. Graphic representations of the contribution of each cluster to the cells belonging to each genotype an of cells of each genotype to every cluster can be found in figure $4.25 \mathrm{D}$ and $\mathrm{E}$.

Next, I asked our collaboration partner Stefan Günther to perform a velocity analysis. This analysis compares mRNA and pre-mRNA levels in a given cell and thereby predicts the direction of cellular differentiation processes depicted as arrows (Figure $\mathbf{4 . 2 5 C}$ ). The analysis suggested that in the mutant a population in cluster 6 contains cells that differentiate into pronephric tubule cells and skeletal muscle. I hypothesized that these cells reflect a progenitor population.

To test this, I plotted early-expressed genes of the npas $4 l$ lineage such as npas $4 l$ expression itself, its direct downstream targets and the early endothelial marker fli1a that is first expressed independently of npas $4 l$ transcriptional activity on UMAP plots only for mutant cells (Figure 4.26). Indeed, the population hypothesized to be a progenitor population expressed npas 4 , fli1a, lmo2, and etsrp but failed to express tal1. It is worth mentioning, though that the expression levels displayed in this graph are scaled for mutant cells only and are incomparable to the levels of expression observed in endothelial progenitors of heterozygous embryos. However, the analysis suggests that npas $4 l$ reporter positive cells develop into skeletal muscle and 

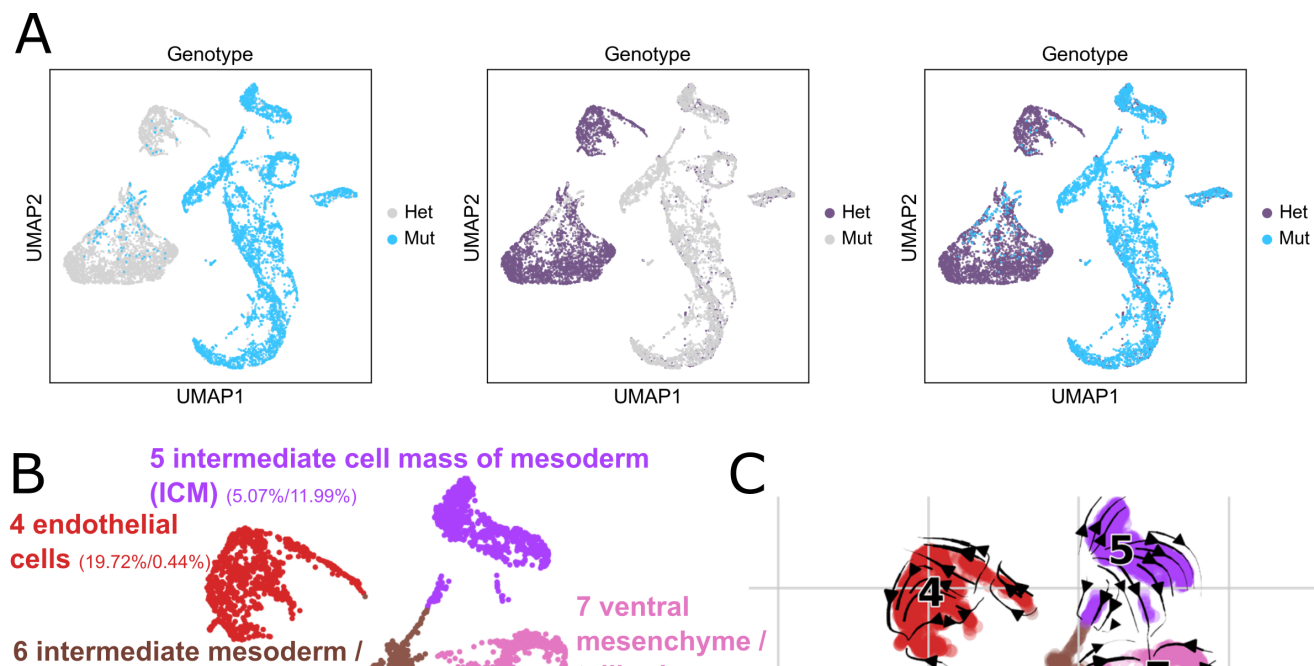

$$
\text { mass of mesoderm }
$$

C pronephric tubules

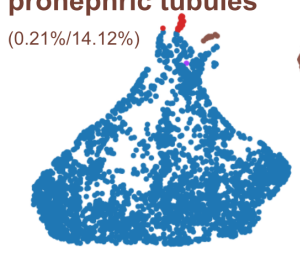

1 blood (58.77\%/2.79\%)
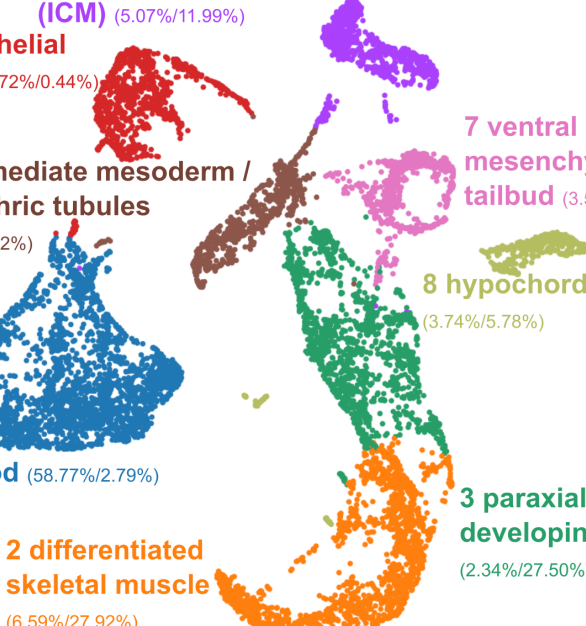

C

D

$$
\begin{aligned}
& \text { npas4l'/- } \\
& \text { npas4l/- }
\end{aligned}
$$

$$
\text { cluster \# 1,2,3,4,5,6,7,8 }
$$

F

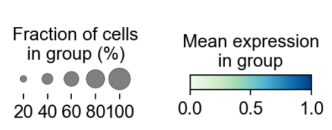

E

3 paraxial mesoderm / developing skeletal muscle (2.34\%/27.50\%)

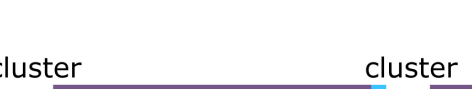

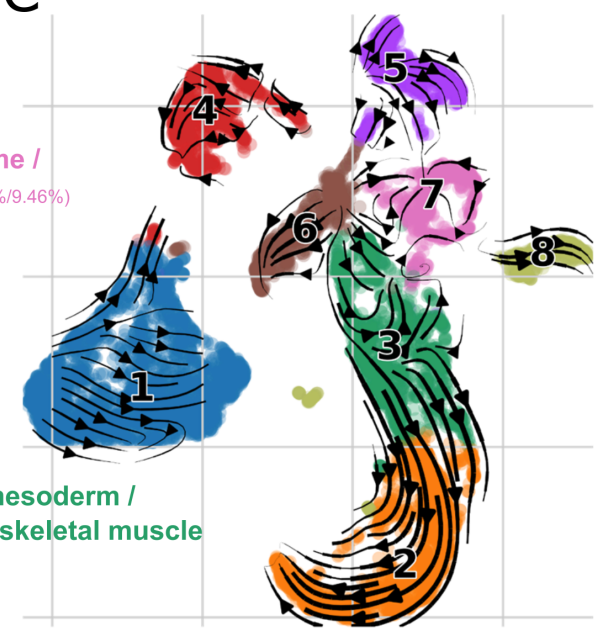
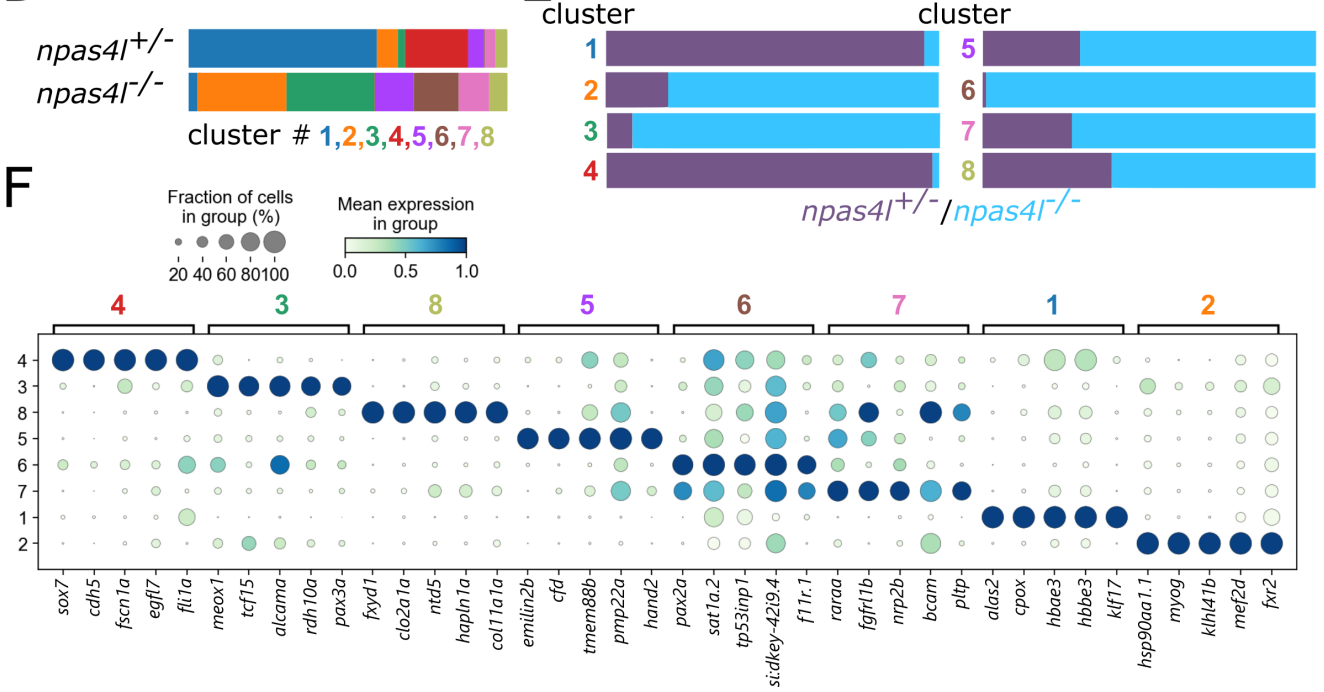

Figure 4.25: Single cell transcriptomic profiling of $n p a s 4 l$ reporter positive cells in $n$ pas $4 l^{+/-}$and $n$ pas $4 l^{-/}$embryos. Figure legend continued on the next page. 
Figure 4.25: (A) 5207 and 5262 npas $4 \mathrm{l}$ reporter expressing cells from the posterior half of $20 \mathrm{hpf} n$ nas $_{4} \mathrm{l}^{+/-}$(purple) and npas4t/- (blue) embryos, respectively, were sequenced using the 10x sequencing platform. The two genotypes show a clear separation reflecting strong differences between the genotypes. (B) UMAP representation of the data clustered by the Leiden algorithm. Numbers in brackets indicate the percentage of heterozygous/mutant cells contributing to the cluster. (C) Velocity analysis done by comparing pre mRNA and mRNA levels to infer the relation between the cells in a cluster. (D) Cluster composition per genotype. Graphic representation of the numbers displayed in (B). (E) Proportional contribution of genotype to the clusters. The main clusters decreasing in npas $4 \mathrm{l}^{-/}$compared with npas $4 l^{+/-}$siblings are endothelial cells and blood cells (clusters 1 and 4 ). The main clusters increasing in npas $4 \mathrm{l}^{-/}$compared with npas $4 l^{+/-}$siblings are paraxial mesoderm (cluster 3), skeletal muscle (cluster 2), and intermediate mesoderm (cluster 6). (F) Dot plot with the top 5 markers separating the clusters. The full annotated dataset can be explored at https://mpibn.mampok.ingress.rancher.computational.bio/dst138-kennyscrnaseq/cellxgene/. I thank Stefan Günther for his help with the library preps, sequencing, quality control, basic analyses and velocity analysis of the data. Plotting and other functions were self-made using the Scanpy package (Wolf et al., 2018).
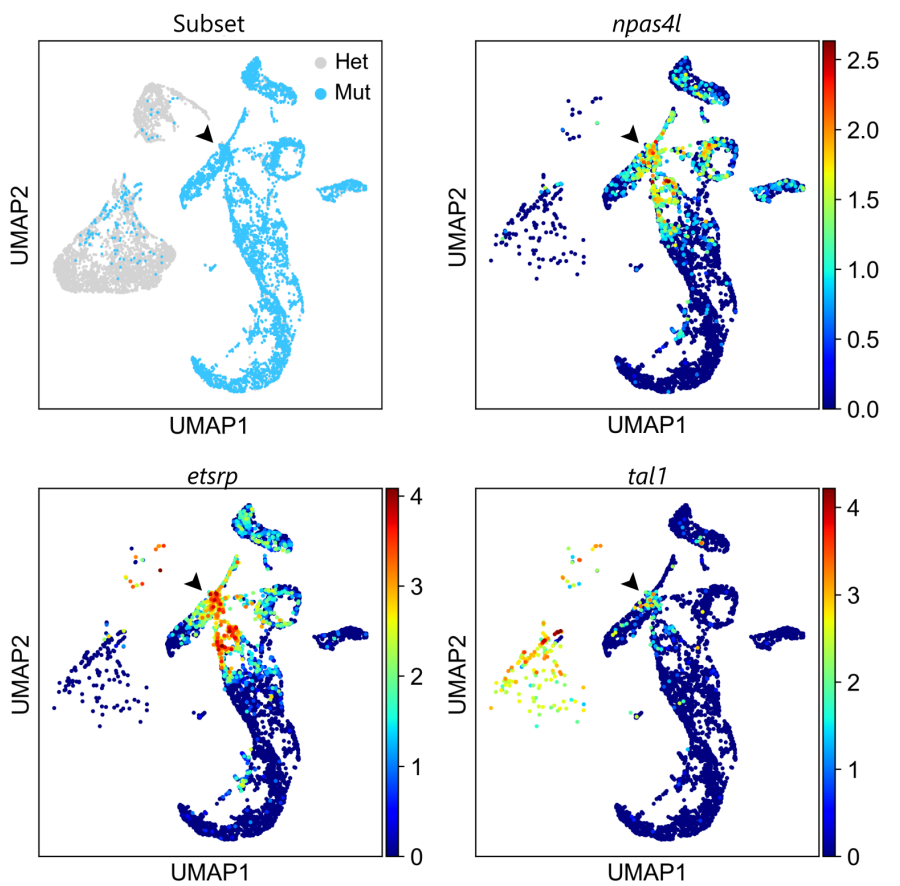
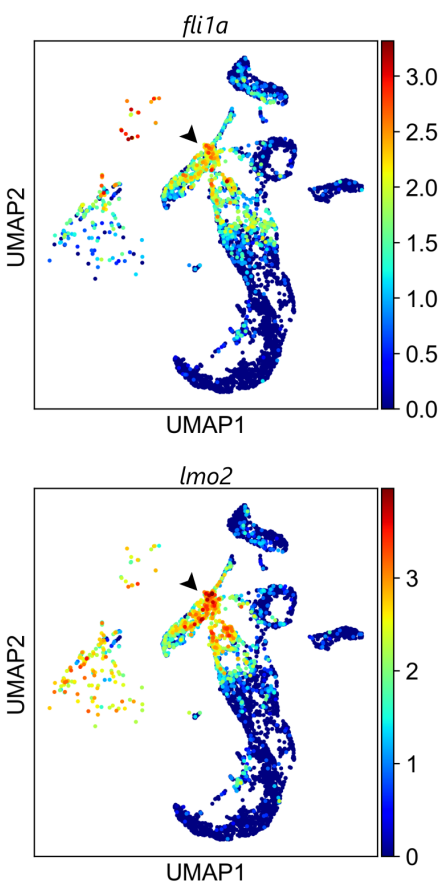

Figure 4.26: A population of mutant cells in cluster 6 expresses npas 4 l transcripts. UMAP plot visualizing the expression of npas4l, etsrp, tal1 and lmo2 as well as of the early endothelial gene fli1a in npas $4 \mathrm{l}^{/-}$embryos. Only mutant cells are shown as indicated in the upper left plot, and the cluster containing the remaining endothelium in npas $4 l^{-/}$embryos is indicated (arrowheads). npas 4 l, etsrp and lmo2 are co-expressed with fli1a. tal1 is not expressed in these cells. 
pronephric tubule cells in the absence of Npas4l function.

A

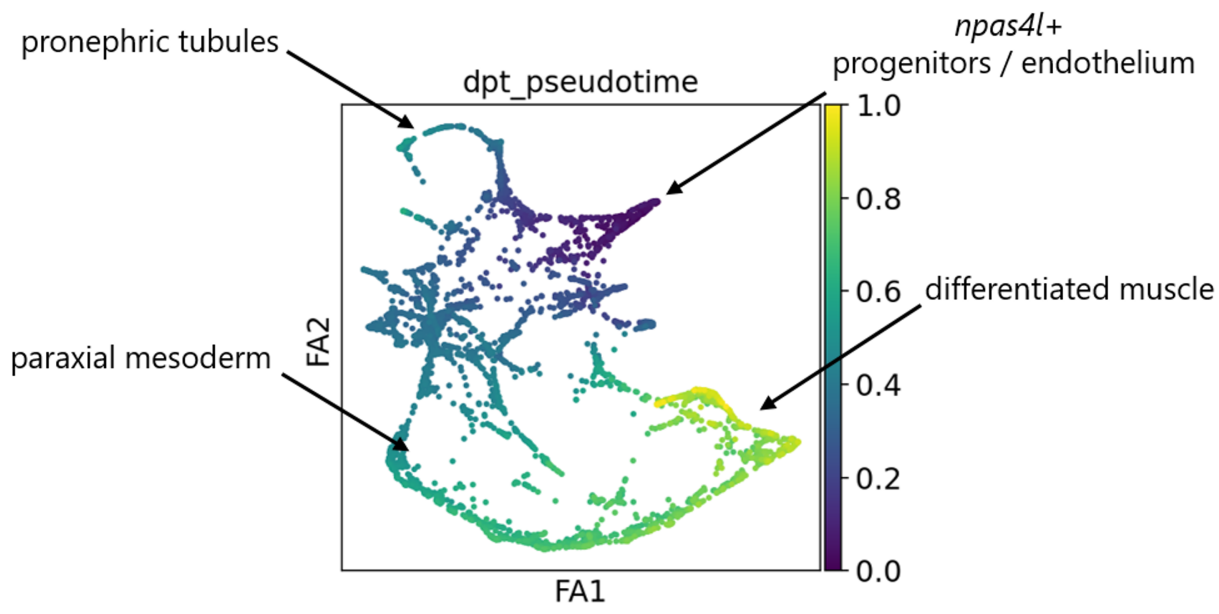

B

npas $4 l+$ population and endothelial markers
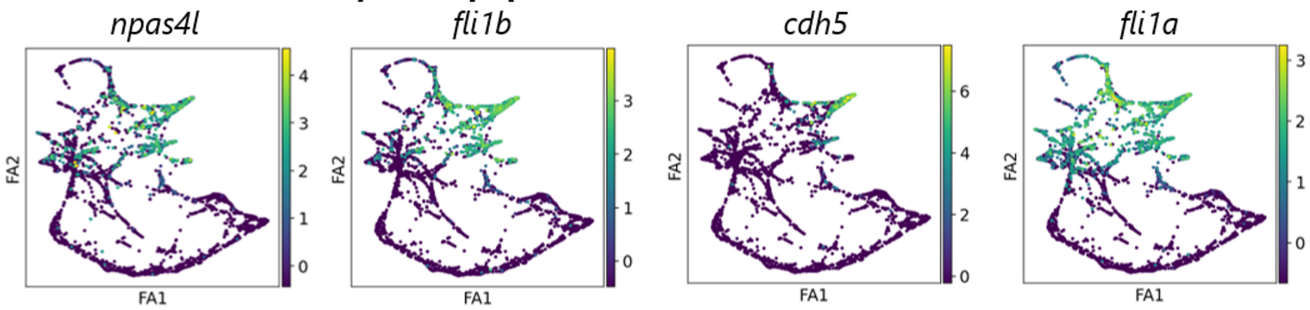

C

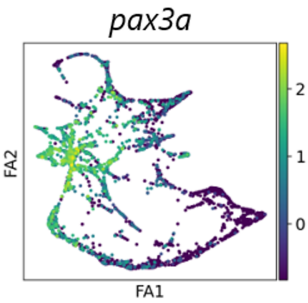

paraxial mesoderm / skeletal muscle markers
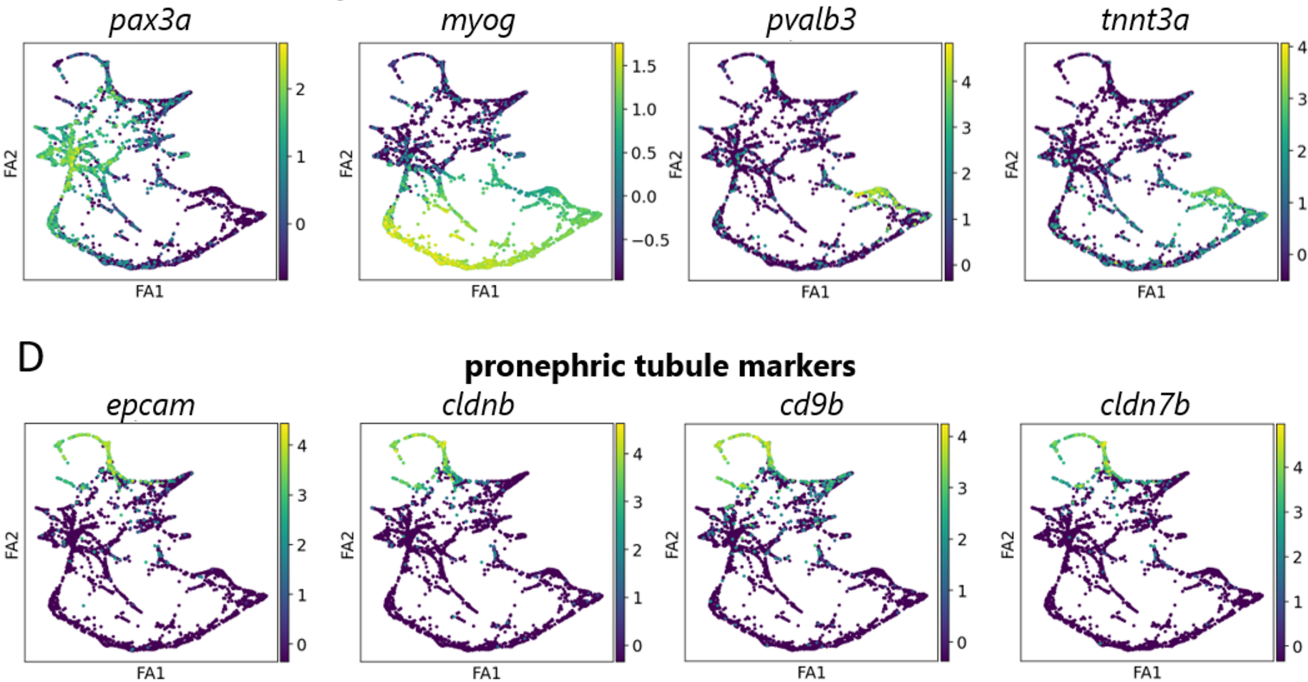

Figure 4.27: Trajectory analysis of npas $4 l$ reporter expressing cells in $n p a s 4 l$ mutants. Figure legend continued on the next page. 
Figure 4.27: (A) Pseudotime analysis suggests that the cells that express npas $4 l$ are the least differentiated and that they differentiate into pronephric tubule and skeletal muscle cells. Louvain clustering displayed as force directed graph generated through ForceAtlas2 (FA). Annotations were added manually as visual aids. (B-D) four marker genes for npas $4 l$ positive progenitors / endothelium (B), pronephric tubule cells (C) and skeletal muscle cells (D). I thank Stefan Günther for performing this trajectory analysis.

The remaining endothelial cells positive for fli1a cluster with early pronephric tubule and skeletal muscle cells suggesting a lineage specification defect. The cells exhibited a transcriptional profile like the one seen in bulk RNAseq of fli1a reporter positive cells suggesting that these cells represent the remaining endothelial cells in cloche/npas 4 l mutants. Interestingly, the lack of tal1 expression in these cells may be the reason for the lack of hemogenic potential and blood marker expression in these mutant endothelial cells.

The velocity analysis predicted the differentiation of npas $4 l$ reporter positive cells into skeletal muscle and pronephric tubule cells. Since this is a prediction however, comparing immature and mature mRNA levels and thereby predicting the future fate of a cell, this analysis is not always reliable. Therefore, I asked Stefan to perform an additional trajectory analysis that predicts the differentiation in pseudotime by comparing the transcriptional profile of cells to their nearest neighbors (Figure 4.27). This approach is technically different from the velocity analysis but resulted in similar conclusions. Based on the pseudotime analysis, npas $4 l$ positive progenitors develop into pronephric tubule cells and paraxial mesoderm/skeletal muscle (Figure 4.27A). The fli1a/cdh5 positive endothelial cells group closely with npas $4 \mathrm{l}$ positive progenitors (Figure $4.27 \mathrm{~B}$ ) and develop subsequently into paraxial mesoderm (Figure 4.27C) and pronephric tubule cells (Figure 4.27D).

All in all, the single cell RNAseq data confirmed the phenotypic data observed and allows us to speculate that npas 4 l positive progenitors that lack Npas4l transcriptional activity fail to express the genes necessary for endothelial specification and instead contribute to skeletal muscle and pronephric tubule cells suggesting a cell-autonomous differentiation defect of the npas $4 \mathrm{l}$ lineage. 


\subsection{Generation of out-of-frame and in-frame mutants for etsrp, tal1 and lmo2}

The phenotypic and molecular analyses of the npas $4 l$ mutant phenotype suggested multiple different defects such as angioblast differentiation, angioblast migration and lineage specification defects of npas $4 l$ positive cells without Npas4l function that resulted in their contribution to pronephric tubule and skeletal muscle.

The commitment of etsrp reporter positive cells to skeletal muscle in etsrp mutants had been observed before, but pronephric tubule cells in these mutants had not been reported (Chestnut et al., 2020). I, therefore, hypothesized that different downstream targets of Npas4l cause different aspects of the phenotype. To test this hypothesis, I decided to generate mutants for the three major direct downstream effectors of Npas4l, etsrp, tal1 and lmo2 to compare them phenotypically.

I designed CRISPR-Cas9 guide RNA's for all three mutants. While Lmo2 mainly acts as a transcription factor scaffold, Etsrp and Tal1 both directly bind to the DNA. Therefore, the targeting reagents were designed to disrupt the DNA-binding site encoding regions of these two genes.

Ideally, this strategy could be used to disrupt only a few aminoacids in the DNA-binding site and still cause a strong mutant phenotype. Such a strategy is beneficial as it leads to minimal interference with the coding sequence and avoids unwanted side effects such as splicing defects and mRNA degradation that could trigger transcriptional adaptation effects and potentially alter the phenotype (Kok et al., 2015; Rossi et al., 2015; El-Brolosy et al., 2019). To disrupt the function of Lmo2 effectively, it was unlikely that a small inframe deletion could lead to a functional null allele as it mainly fulfills a scaffolding role. Therefore, I designed reagents that led to a separation of the two symmetrical protein domains that form its structure. This way, the scaffolding activity of Lmo2 should be disturbed.

I found effective guides targeting the region of interest as determined by high resolution melt analysis (HRMA, Figure 4.28A). Founder screening of only a small number of fish per allele was necessary to recover in-frame and out-of-frame alleles for each gene to establish the mutant lines required for the planned analyses (Figure $4.28 \mathrm{~B}$, screening data not shown). 

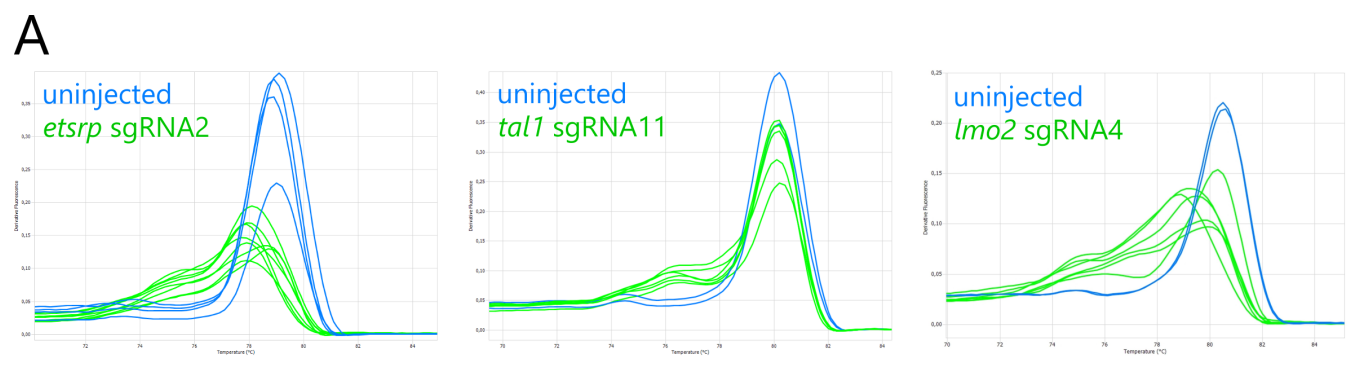

B

etsrp (ENSDARG00000053868.5)

255

ETS-domain

260

265

270

wild-type GCTGGATTCTGCTTGCCACACTTTTATAAGTTGGACTGGTGATGGCTGGGAGTTTAAA

*bns426 (-9 bp) GCTGGATTCTGCTTGtCACACTTTTATAAGTTGGACTGGTGA---_-----GTTTAAA bns422 (-4 bp) GCTGGATTCTGCTTGCCACACTTTTATAAGTTGGACTGG----GGCTGGGAGTTTAAA

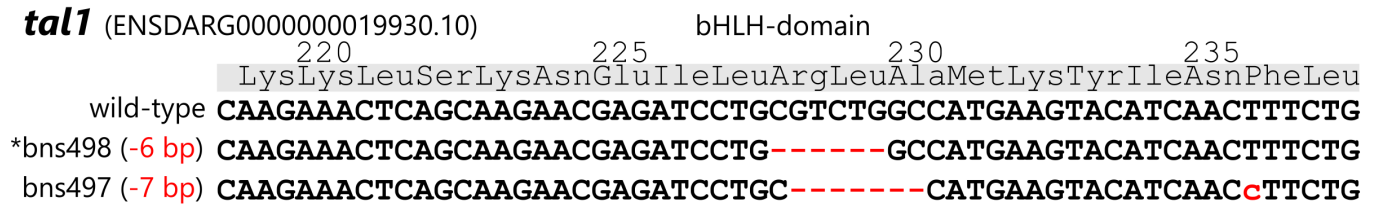

Figure 4.28: Injection validation and sequences of the etsrp, tal1 and lmo2 alleles. (A) High resolution melt analysis of uninjected controls and injected P0 embryos for all three genes targeted for mutations validates the efficiency of these strategies. (B) Partial sequence of one in-frame $\left(^{*}\right)$ and one out-of-frame indel per gene.

\subsection{4 etsrp, tal1 and lmo2 mutants show dis- tinct phenotypes}

As a first experiment, I compared the mutant phenotypes of the in-frame and out-of-frame alleles qualitatively and by counting ISV numbers. For etsrp (Figure 4.29A-A') and tal1 mutants (Figure 4.29B-B') the phenotypes of in-frame and out-of-frame mutants looked comparable. Although the p-value in figure 4.29A' suggests a significant difference, the statistical power of the experiment is too low and the variability in the data too high to quantify such a minor effect reliably. Both lmo2 mutants lacked blood cells (data 

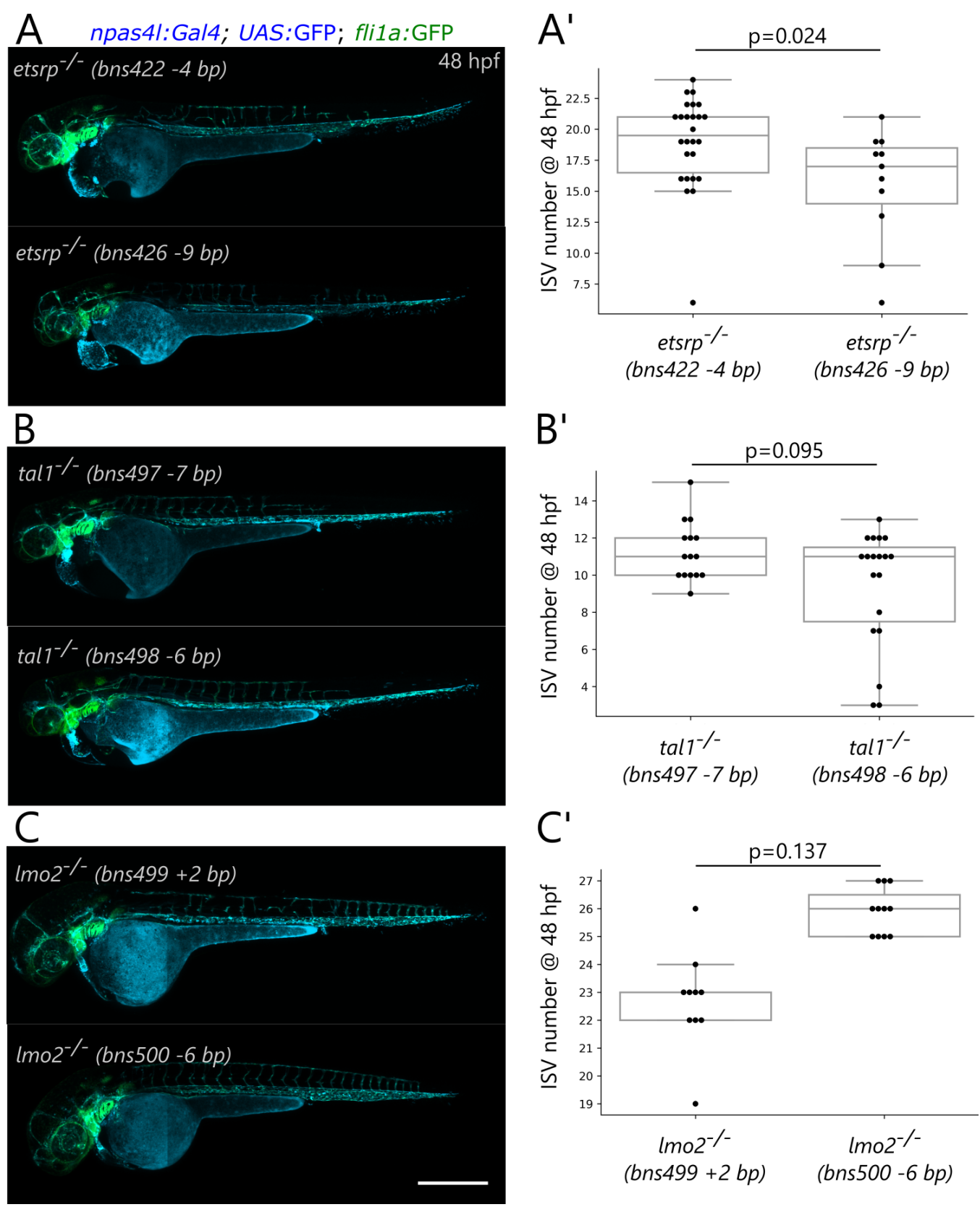

Figure 4.29: Phenotypic comparison of the etsrp, tal1 and lmo2 mutant alleles. (A-C) For etsrp and tal1, the in-frame and out-of-frame alleles exhibit similar phenotypes, while for lmo2, the out-of-frame allele exhibits mild vascular defects which are not observed in the in-frame allele. (A'-C') Quantifications of ISV numbers in homozygous carriers of all 6 mutant alleles. Data are represented as individual datapoints, median, interquartile range and extremes excluding outliers. p values were calculated by Poisson regression. Scale bar: $500 \mu \mathrm{m}$. 
not shown), but vascular defects as determined by missing ISV were only observed in the out-of-frame-allele (Figure 4.29C-C'). Again, the sample size was too low to quantify this moderate effect, although it was reliably observed in all mutant animals and crosses. As this experiment was only an informal experiment to choose the best allele to work with and was limited by the number of mutant embryos obtained from the crosses, the quantifications were not repeated at later time points.

Based on these comparisons, I chose to work with the in-frame alleles etsrp ${ }^{\text {bns426 }}$ and tal1 ${ }^{\text {bns498 }}$ as well as the out-of-frame allele $l m o 2^{b n s 499}$ as representative mutant alleles for these genes. The changes to the coding sequences caused by these alterations are displayed in figure $\mathbf{4 . 3 0}$.

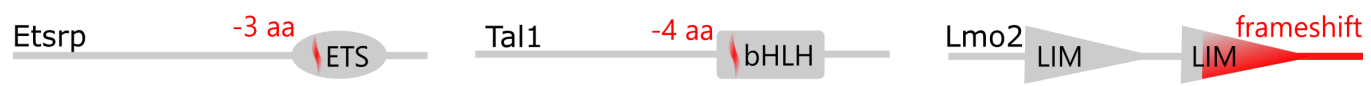

Figure 4.30: Schematic representation of the protein structure of the mutants selected for this project. The changes in the peptide sequence caused by the in-frame deletions for etsrp and tal1 as well as the out-of-frame deletion for lmo2 are highlighted in red.

Published phenotypes for strong mutants of npas4l, tal1 and lmo2 include a lack of red blood cells as determined by a loss of gata1a expression throughout the embryo, whereas etsrp mutants do not show a lack of gata1a positive red blood cells. To test for this phenotype as a validation of a strong mutant phenotype, I performed wholemount in situ hybridization on all those mutants and looked for gata1a positive cells in the tail region. As expected, only npas $4 l$ heterozygous and etsrp mutant embryos showed gata1a expression in the tail whereas homozygous mutants for npas4l, tal1 and lmo2 did not show such expression (Figure 4.31). It is interesting to note that in npas 4 , tal1 and lmo2 mutants, a very faint fluorescence of gata1a transgenic reporters can be observed in the pronephric ducts at $24 \mathrm{hpf}$ (data not shown), indicating an initial expression of early blood markers but a failure to differentiate into blood cells and a consequent commitment to the pronephric tubules.

Next, I compared all these mutants side by side in transverse sections (Figure 4.32). In heterozygous fish, the main axial as well as the intersomitic vessels had already formed by $24 \mathrm{hpf}$. In npas $4 \mathrm{l}$ mutants, angioblast precursors did not migrate or specify. In etsrp mutants, migration towards the midline was apparently not affected, but the cells reaching the midline remained rounded and failed to express endothelial markers such as fli1a. In tal1 mutants, many cells did not migrate towards the midline but remained 


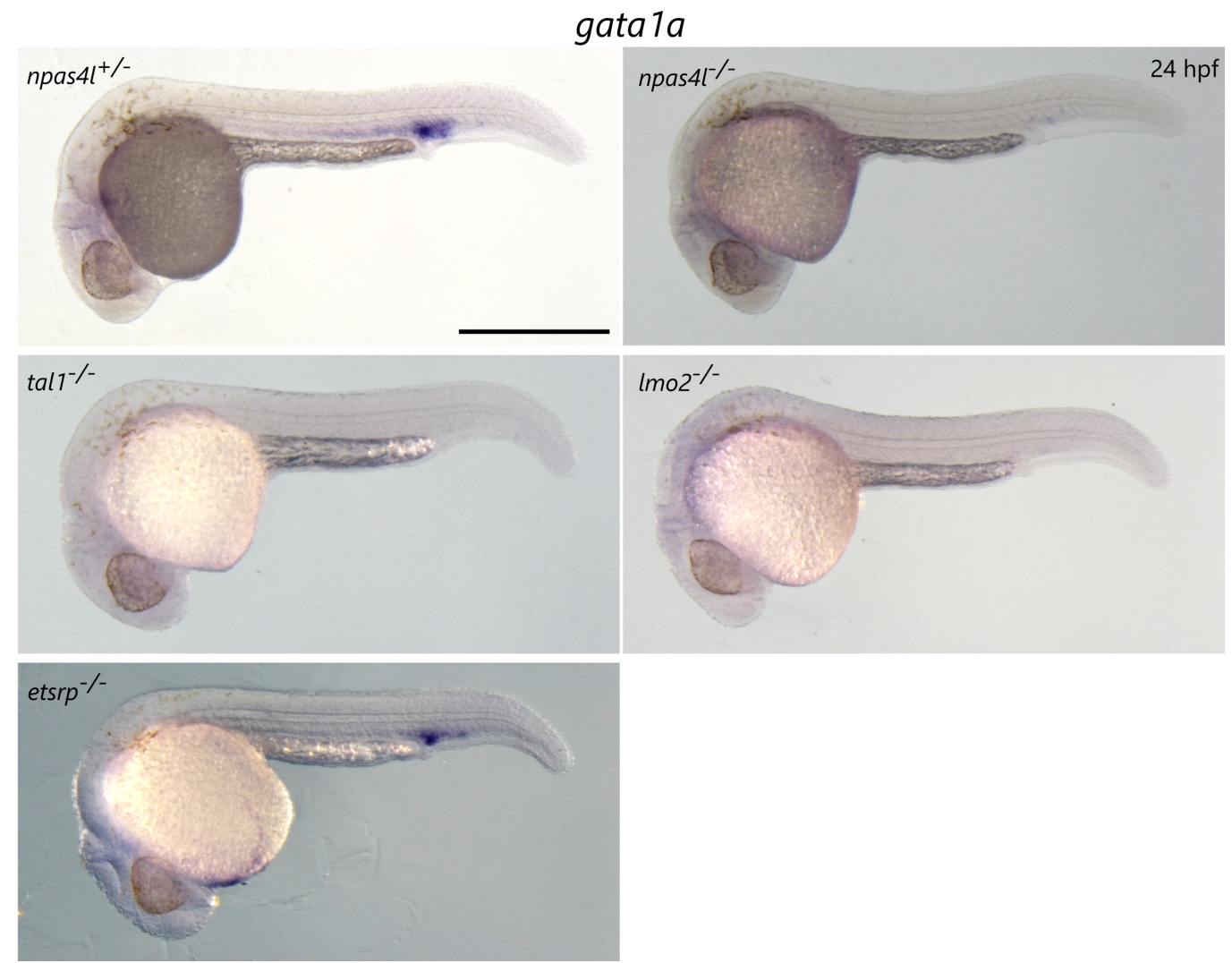

Figure 4.31: gata1a in situ hybridization. gata1a positive cells in the trunk are

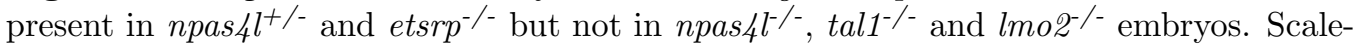
bar: $500 \mu \mathrm{m}$.

lateral like in npas $4 l$ mutants. Those npas $4 l$ reporter positive cells that did reach the midline, developed normal endothelial characteristics and expressed endothelial markers. A phenotype that was overall milder but similar to the one of tal1 mutants was observed in lmo2 mutants.

To test for an increase in skeletal muscle and pronephric tubule cells in the different mutants, I imaged wholemount immunostainings against the npas 4 l reporter and Pax2a (Figure 4.33). The data suggest an increased number of npas $4 \mathrm{l}$ reporter positive skeletal muscle cells in npas $4 \mathrm{l}$ and etsrp mutants but not in tal1 and lmo2 mutants. In contrast, an increased contribution to the pronephric tubules was observed in npas $4 l$ and tal1 mutants but not in etsrp mutants. lmo2 mutants showed a variable phenotype but could have a slightly increased number of npas4l reporter positive pronephric tubule cells as well. 


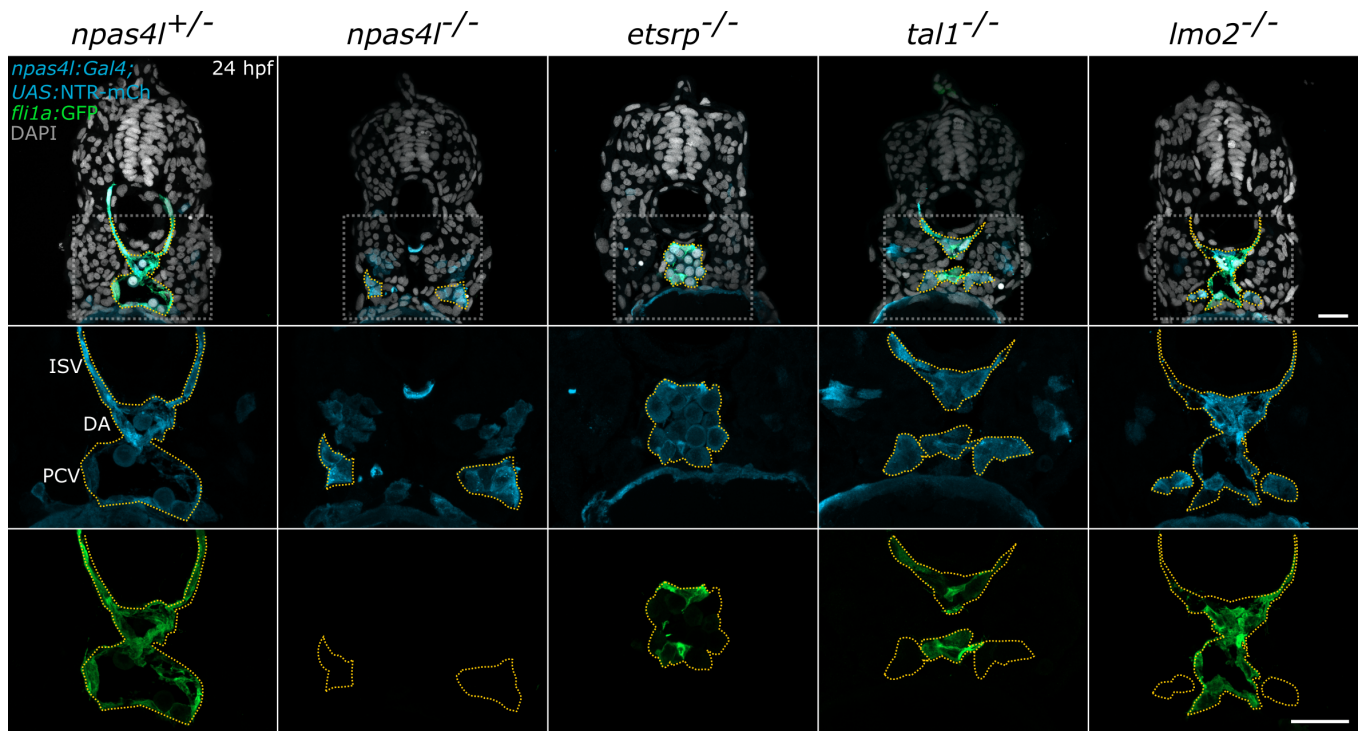

Figure 4.32: Endothelial progenitors exhibit distinct defects in npas4l, etsrp, tal1 and lmo2 mutants. Transverse sections of npas $4 \mathrm{l}^{+/-}$, npas $_{4} \mathrm{l}^{--}, \mathrm{esrp}^{-/}, \mathrm{tal1}^{-/-}$and lmo2 $2^{-/}$embryos at $24 \mathrm{hpf}$. Endothelial progenitors and endothelial cells outlined by yellow dotted lines. Major axial vessels form in npas $4 l^{+/-}$embryos, while in npas $4 l^{/-}$embryos endothelial progenitors fail to migrate or to express the fii1a:GFP reporter. In etsrp ${ }^{-/}$ embryos, endothelial progenitors migrate but fail to form vessels. In $\mathrm{tal1}^{1 /-}$ and $\mathrm{lmo}^{-2^{-/}}$ embryos, endothelial progenitor migration is partly impaired; the endothelial progenitors that reach the midline differentiate and express fli1a:GFP. Scale bar: $20 \mu \mathrm{m}$.

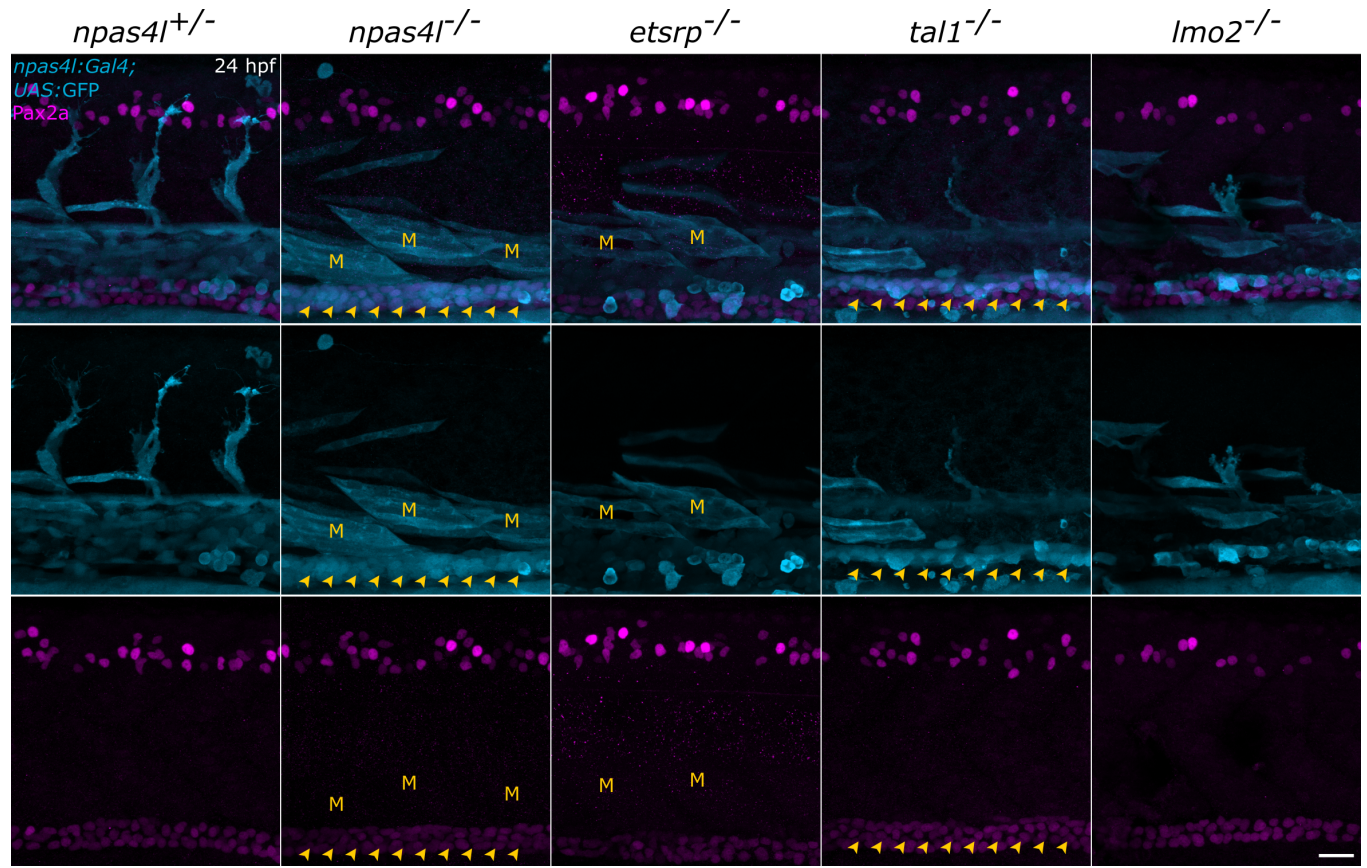

Figure 4.33: npas $4 l$ reporter positive cells contribute to different lineages in etsrp and tal1/lmo2 mutants. npas4l reporter positive cells in npas $4 l^{/-}$and tal1 ${ }^{-/}$ embryos overlap with a high number of Pax2a positive pronephric tubule cells (yellow arrowheads). More npas $4 l$ reporter expressing skeletal muscle cells (M) are observed in npas $4 t^{/-}$and $\mathrm{etsrp}^{-/-}$embryos than in tal1 $^{-/}$and $l \mathrm{mo}^{-/-}$embryos. Scale bar: $20 \mu \mathrm{m}$. 
Pax2a also appeared to be expressed in some of the cells that reached the midline in several mutants, especially in lmo2 mutants. To validate these observations, I performed immunostainings for a fli1 a transgenic reporter and Pax2a. In lmo2 mutants, a high number of Pax2a positive endothelial cell in the posterior cardinal vein region were observed (Figure 4.34). These results were especially interesting given the lower number of lateral npas $4 \mathrm{l}$ reporter positive pronephric tubule cells observed in lmo2 mutants compared to tal1 and npas $4 l$ mutants. Based on these experiments, endothelial progenitors may be able to migrate towards the midline in lmo2 mutants but still show signs of lineage specification defects like the ones observed in npas $4 l$ and tal1 mutants.

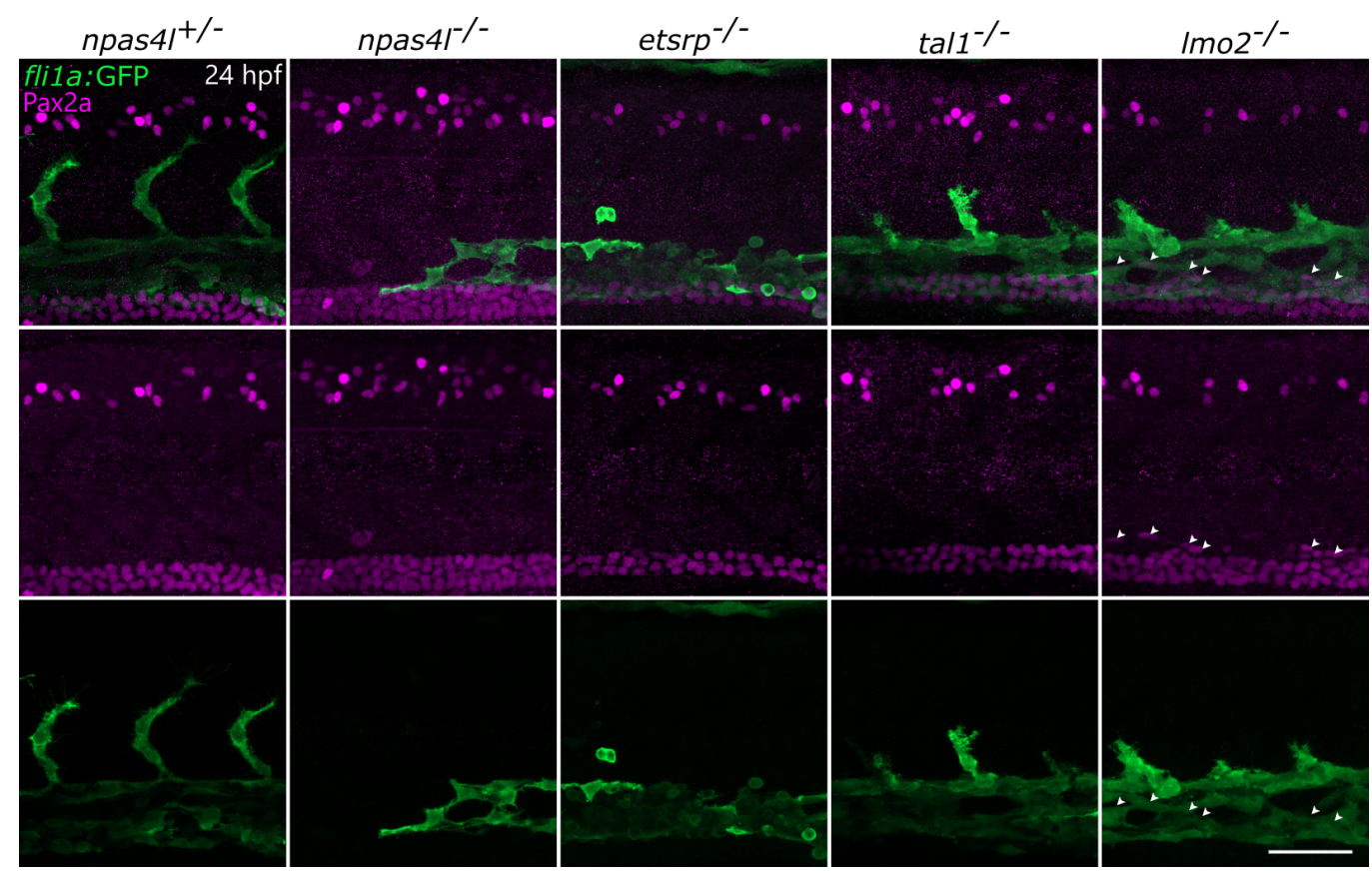

Figure 4.34: fli1a/Pax2a double positive endothelial cells are present in high numbers in $\mathrm{lmo}^{-/-}$embryos. Pax2a and GFP immunostaining of npas $4 \mathrm{l}^{+/-}$, npas $4 \mathrm{l}^{-/}$, etsrp $^{-/-}$, tal1 $^{-/-}$and $l m o 2^{-/-}$embryos at $24 \mathrm{hpf}$. Some fli1a reporter positive cells in the PCV of $l m o 2^{-/-}$embryos are also Pax2a positive (arrowheads). Scale bar: $20 \mu \mathrm{m}$.

In summary, I observed distinct phenotypic differences between tal1, etsrp and lmo2 mutants. In etsrp mutants, endothelial progenitors migrate to the midline but fail to express vascular markers. Some also contribute to the paraxial mesoderm-derived skeletal muscle. In tal1 mutants, endothelial progenitors fail to migrate towards the midline and instead contribute to the pronephric tubules. Cells that reach the midline express endothelial markers and acquire endothelial characteristics. In lmo2 mutants, endothelial pro- 
genitors manage to migrate towards the midline, but express the pronephric tubule marker Pax2a, suggesting lineage specification defects similar to the ones observed in tal1 mutants. npas 4 l mutants show a combination of all defects observed in its three downstream mutants, combining into an overall stronger phenotype.

\subsection{5 npas4 $\mathrm{l}^{-/-} ;$etsrp $^{-/-}$and tal1//- etsrp $^{-/-}$dou- ble mutants lack endothelial cells com- pletely}

The preliminary experiments suggested that the strong cloche/npas $4 l$ phenotype is caused by a combined deficiency in etsrp and tal1 expression. The loss of Etsrp function explains the skeletal muscle contribution and endothelial differentiation defects, whereas the loss of Tal1 function explains the endothelial migration defects, the loss of red blood cells and the strong increase in npas4l reporter positive pronephric tubule cells. I hypothesized that double mutants for etsrp and tal1 display a stronger phenotype indistinguishable from the npas4l mutant phenotype.

Against expectations, etsrp ${ }^{-/} ;$tal1 $^{-/-}$double mutants showed an even stronger phenotype than the npas $4 l$ mutant phenotype characterized by a complete loss of endothelial cells in the mutant embryos (Figure 4.35), making it the strongest endothelial phenotype ever observed in vertebrates. This raised the question why it was even stronger than the npas 4 l mutant phenotype. To test whether remaining endothelial cells in the npas $4 l$ mutants develop because of residual expression of either tal1 or etsrp independent of Npas4l transcriptional activity, I generated double mutants for $n$ pas $4 \mathrm{l}^{-/-} ; \mathrm{tal1}^{-/-}$and npas $4 \mathrm{l}^{-/-} ;$etsrp $^{-/-}$as well. While npas $4 \mathrm{l}^{-/} ;$tal1 $^{-/-}$double mutants did not show stronger phenotypes compared to npas $4 \mathrm{l}$ mutants alone, npas $4^{-/} ;$etsrp $^{-/}$ double mutants displayed the same complete absence of endothelial cells that had been observed in etsrp ${ }^{-/} ;$tal1 $^{-/-}$mutants.

These data fit to results presented earlier in the transcriptomics data (Figure 4.26) that showed etsrp but not tal1 expression in remaining fli1a positive cells of npas 4 l mutants. Combined, these data suggest that etsrp but not tal1 can be expressed in some endothelial progenitors independent of Npas4l transcriptional activity. No endothelial cells develop independent of both Etsrp and Tal1 function. 
npas4l:Gal4; UAS:NTR-mCherry; fli7a:GFP
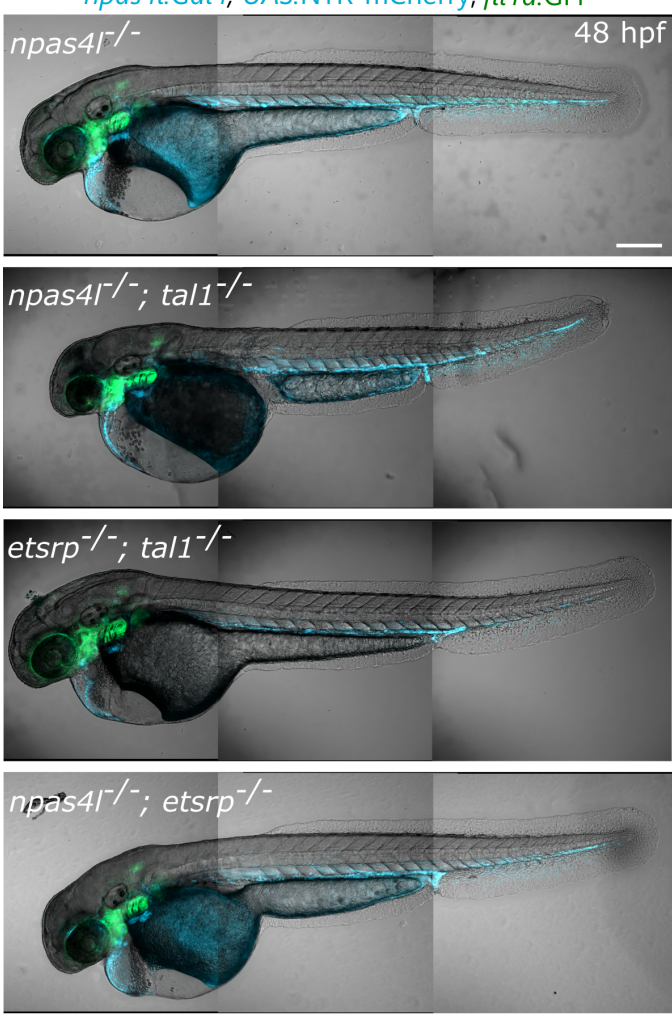
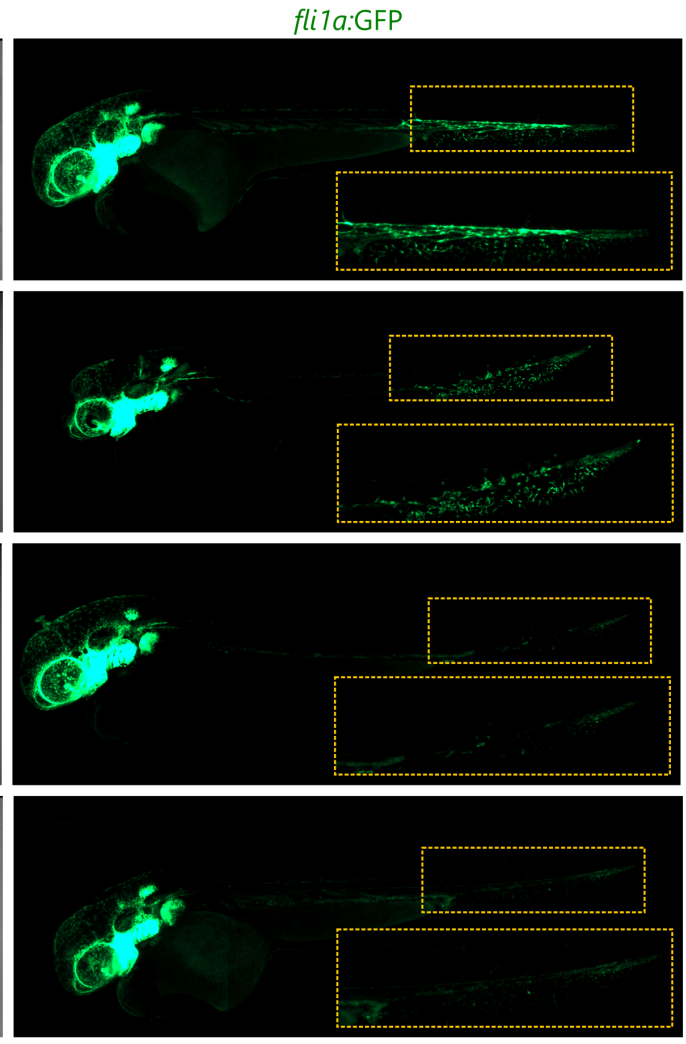

Figure 4.35: npas4t I $^{--} ;$etsrp $^{-/-}$and tal1 $^{-/-} ;$etsrp $^{-/-}$embryos completely lack endothelial cells. At $48 \mathrm{hpf}$, some endothelial cells are visible in $n$ pas $4^{-/-}$embryos but not in npas4t/- etsrp $^{-/-}$or etsrp ${ }^{-/-} ;$tal1 $^{-/-}$embryos. Scale bar: $200 \mu \mathrm{m}$.

\subsection{6 tal1-mRNA overexpression rescues spe- cification of endothelial cells in npas $4 l$ mutants}

The experiments shown so far indicate that effects mediated by Etsrp and Tal1 together explain all known phenotypic aspects of the cloche mutant phenotype. I wanted to know whether overexpression of each factor might rescue the parts of the npas $4 l$ mutant phenotype that have been observed in its own mutant. I also hypothesized that overexpression of both factors combined could lead to a rescue similar to the one achieved with npas $4 \mathrm{l}$ overexpression.

To test this, I synthesized mRNA for npas4l, etsrp, tal1 and lmo2, optimized the injected amounts by balancing effectiveness with severe develop- 

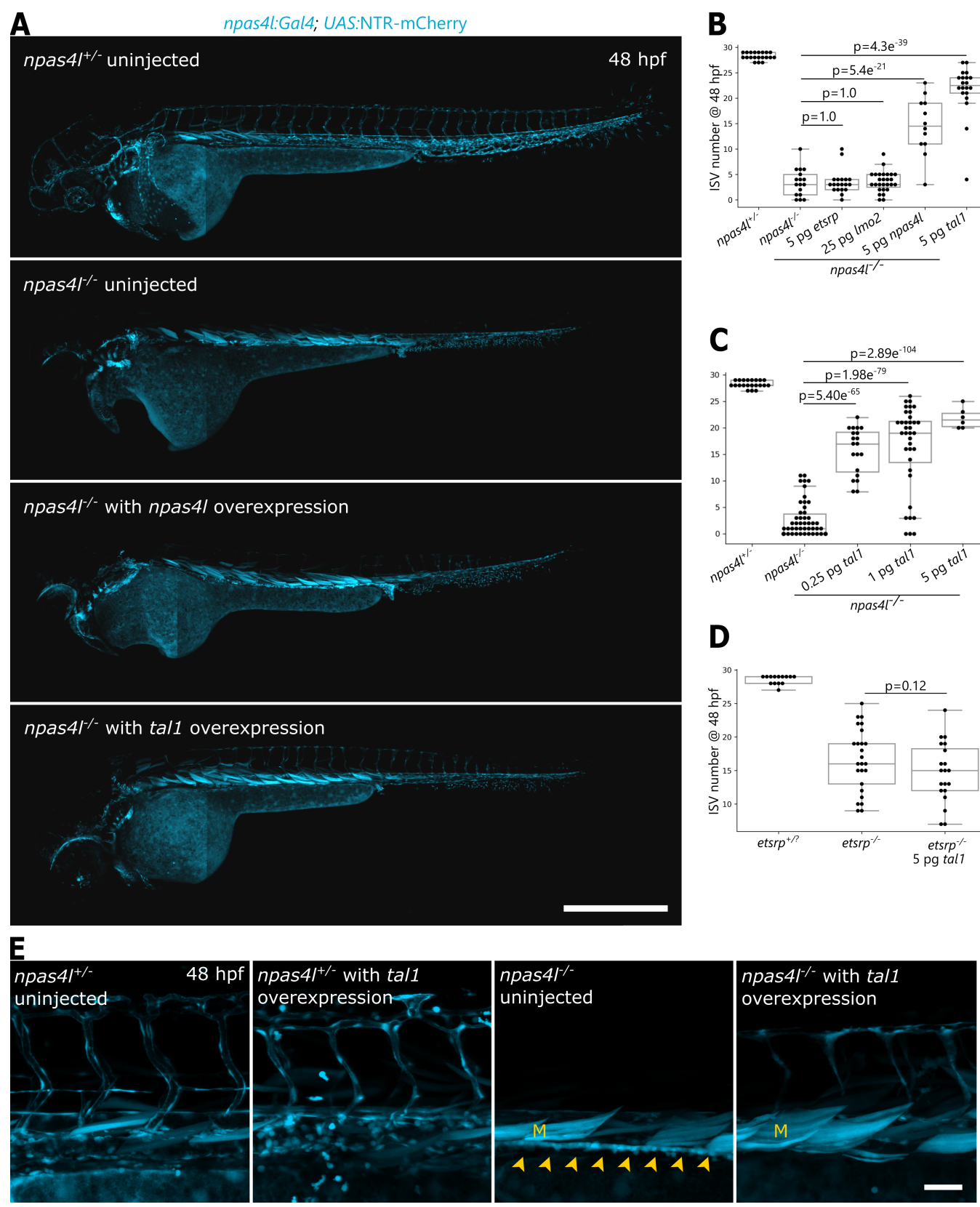

Figure 4.36: tal1 overexpression rescues vasculogenesis in npas $4 l$ mutants in an Etsrp-dependent manner. Figure legend continued on the next page.
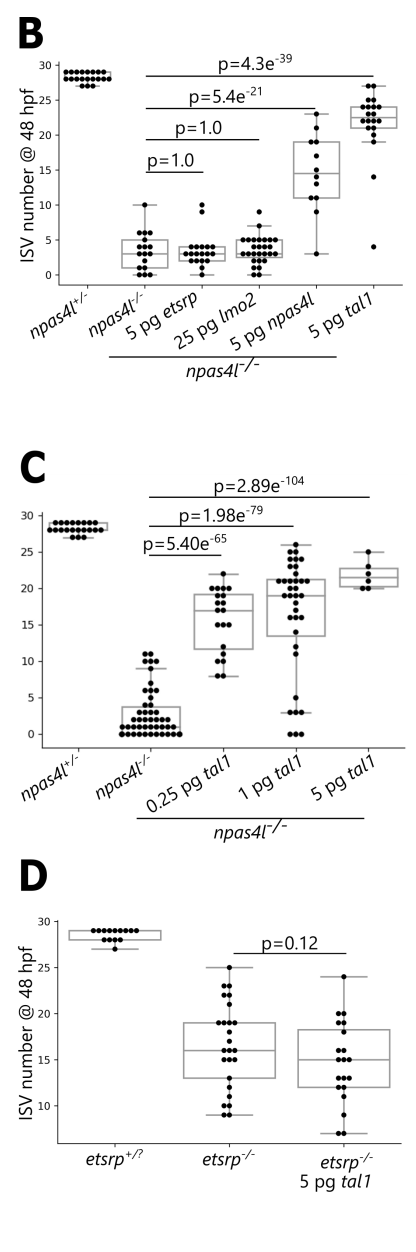
Figure 4.36: (A) npas $4 l$ and tal1 mRNA injections rescue endothelial defects in npas $4 l$ mutants. (B) Quantification of rescue: mRNA injections of npas $4 l$ and tal1 but not of etsrp or lmo2, rescue the ISV defects in npas $4 t^{--}$embryos. (C) The rescue is dosedependent and works with tal1 mRNA amounts as little as $0.25 \mathrm{pg}$. Smaller concentrations have not been tested. (D) tal1 mRNA injections do not rescue the vascular phenotype in etsrp $^{-/-}$embryos. (E) Uninjected and tal1 mRNA injected npas $4 l^{+/-}$and npas $4 t^{/-}$ embryos. Ventrolateral npas $4 l$ reporter positive cells (arrowheads) are not present in tal1 mRNA injected npas $4 l^{-/}$embryos, while the contribution of npas 4 l reporter positive cells to skeletal muscle (M) does not appear to be affected. Scale bars: $500 \mu \mathrm{m}(\mathrm{A}), 50 \mu \mathrm{m}(\mathrm{F})$. Data are represented as individual datapoints, median, interquartile range and extremes excluding outliers. $\mathrm{p}$ values were calculated by Poisson regression. $\mathrm{p}$ values were adjusted using the Bonferroni correction to counteract the multiple testing problem.

mental defects caused by the treatment (data not shown) and then injected these factors into npas 4 l mutants. As a readout for a functional rescue, I chose to quantify the number of ISV present in the uninjected and injected mutants. While injections of etsrp and lmo2 mRNA did not affect ISV patterning at $48 \mathrm{hpf}$, injections of npas $4 \mathrm{l}$ and tal1 positively influenced endothelial development as determined by an increased ISV number (Figure $4.36 \mathrm{~A}$ and $\mathbf{B}$ ). Interestingly, this effect was more pronounced and less variable after tal1 injections compared to npas4l injections with some tal1-mRNA injected animals displaying an almost complete rescue of trunk vasculogenesis and ISV sprouting (Figure 4.36B).

This strong effect was dose-dependent and led to significant effects with a high effect size with doses as little as $0.25 \mathrm{pg}$ of tal1 mRNA. To address whether the rescue effect was dependent or independent of Etsrp, I injected tal1 mRNA into etsrp mutants. Injections of tal1 mRNA did not have a rescuing effect on the phenotype and ISV number in these mutants (Figure 4.36C).

Minutely analyzing the phenotype, I noticed that in tal1 injected npas $4 l$ mutants, npas 4 l reporter positive pronephric tubule cells were no longer detected whereas the number of npas $4 l$ reporter positive skeletal muscle cells was unchanged by the treatment, indicating, that this specific lineage specification defect likely mediated by Etsrp cannot be reversed by rescuing npas $4 \mathrm{l}$ mutants with tal1 mRNA (Figure 4.36E). Another aspect of the npas $4 \mathrm{l} \mathrm{mu-}$ tant phenotype the treatment did not rescue was the presence of the major brain vessels. 


\begin{tabular}{|c|c|c|c|c|c|c|c|}
\hline & npas4l & tal1 & etsrp & $\operatorname{lmo} 2$ & \multicolumn{3}{|c|}{ Legend: } \\
\hline uninjected & 0 & 0 & 0 & 0 & 0 & no significant cha & ge $(p>=0.05)$ \\
\hline $5 p g$ npas4l & 0 & ++ & ++ & + & + & $<10$-fold up & \\
\hline $5 \mathrm{pg}$ tal1 & - & ++++ & + & 0 & $\begin{array}{l}++ \\
+++\end{array}$ & $\begin{array}{l}>10<100 \text {-fold } \\
>100<1000 \text {-fold }\end{array}$ & $-n<0,05$ \\
\hline 5 pg etsrp & - & 0 & ++ & $\mathrm{o}$ & ++++ & $>1000$-fold up & כ \\
\hline $25 \mathrm{pg} \operatorname{lmo} 2$ & 0 & 0 & 0 & ++++ & - & down & \\
\hline
\end{tabular}
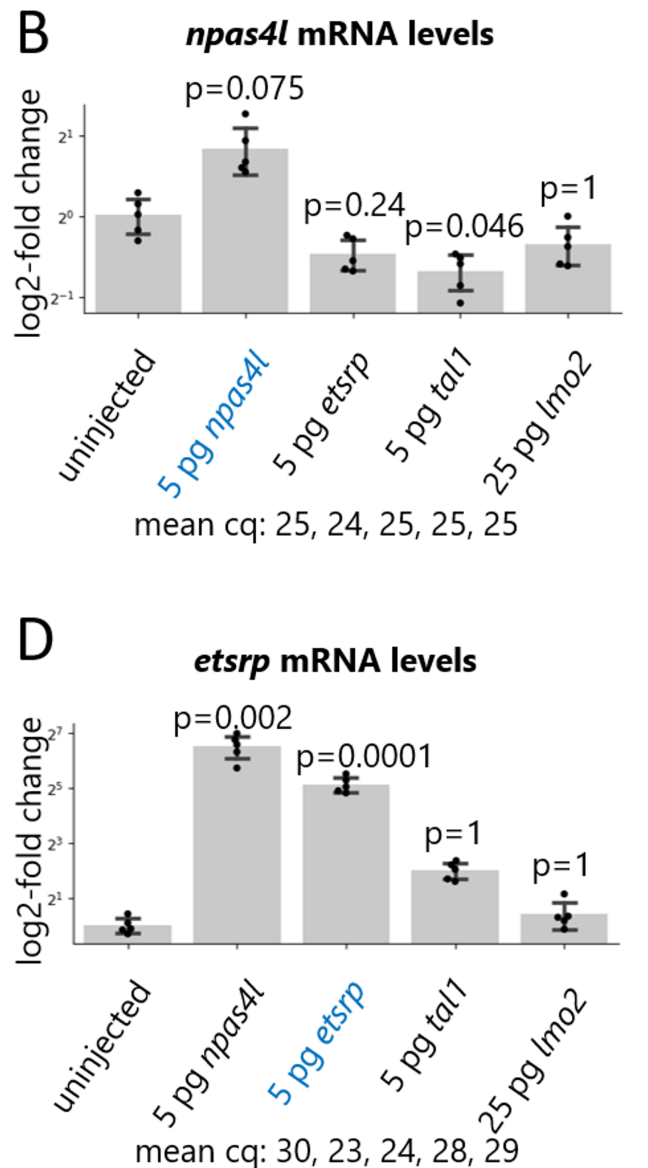
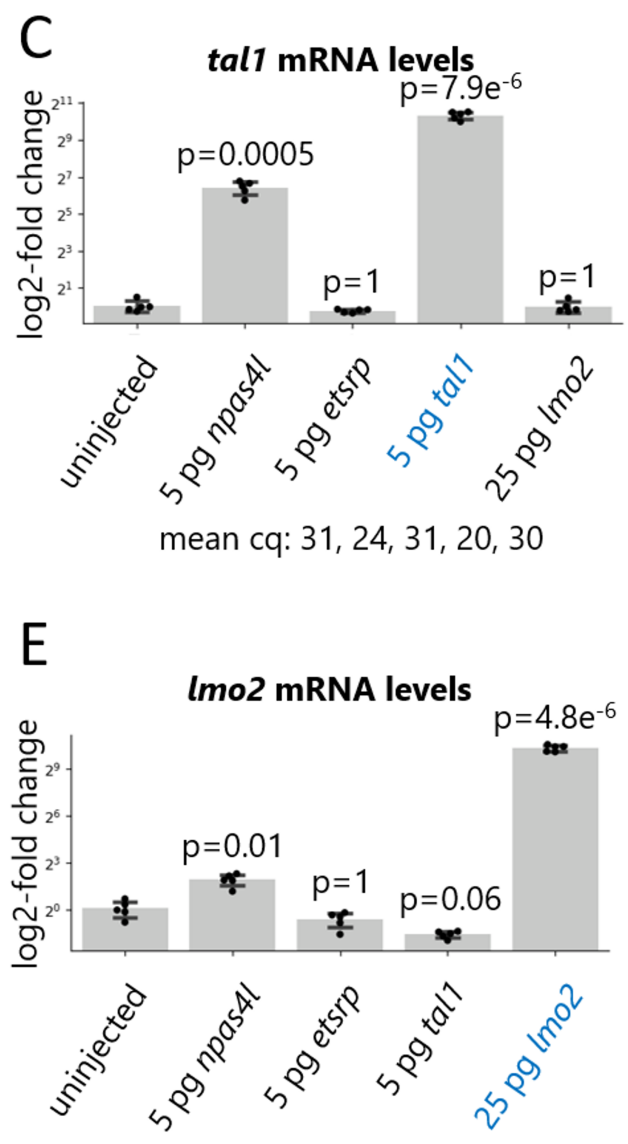

mean cq: $28,26,28,29,17$

Figure 4.37: npas4l, tal1, etsrp and lmo2 mRNA levels at tailbud stage after mRNA injection of each of these factors. Figure legend continued on the next page. 
Figure 4.37: (A) Table summarizing all data points. (B-E) mRNA levels of each gene after injecting mRNA of itself or of the other genes. Detection of the injected mRNA itself by qPCR is highlighted in blue. p-values were adjusted to counteract the multiple testing problem. Data are represented as individual data points as well as mean $\pm \mathrm{SD}$. pvalues were calculated by unpaired two-sample ttest and adjusted for multiple comparisons between five groups using the Bonferroni correction.

As the rescue of npas 4 l mutants by tal1 mRNA injection depended on etsrp expression, I wondered whether tal1 overexpression could increase levels of etsrp. To investigate this, I injected npas4l, tal1, etsrp and lmo2 into wild-type embryos and tested the expression levels of each of those factors at tailbud stage (Figure 4.37). The data summarized in figure 4.37A suggests that npas 4 l injection induces all three other factors. This is in line with the current model that Npas4l drives the expression of these factors (Marass et al., 2019). Interestingly though, tal1 injections led to a slight increase in etsrp mRNA levels, which may be sufficient to start endothelial specification.

A side observation was that both etsrp and tal1 mRNA injections decreased the levels of npas $4 \mathrm{l}$ mRNA at tailbud stage, hinting at a potential
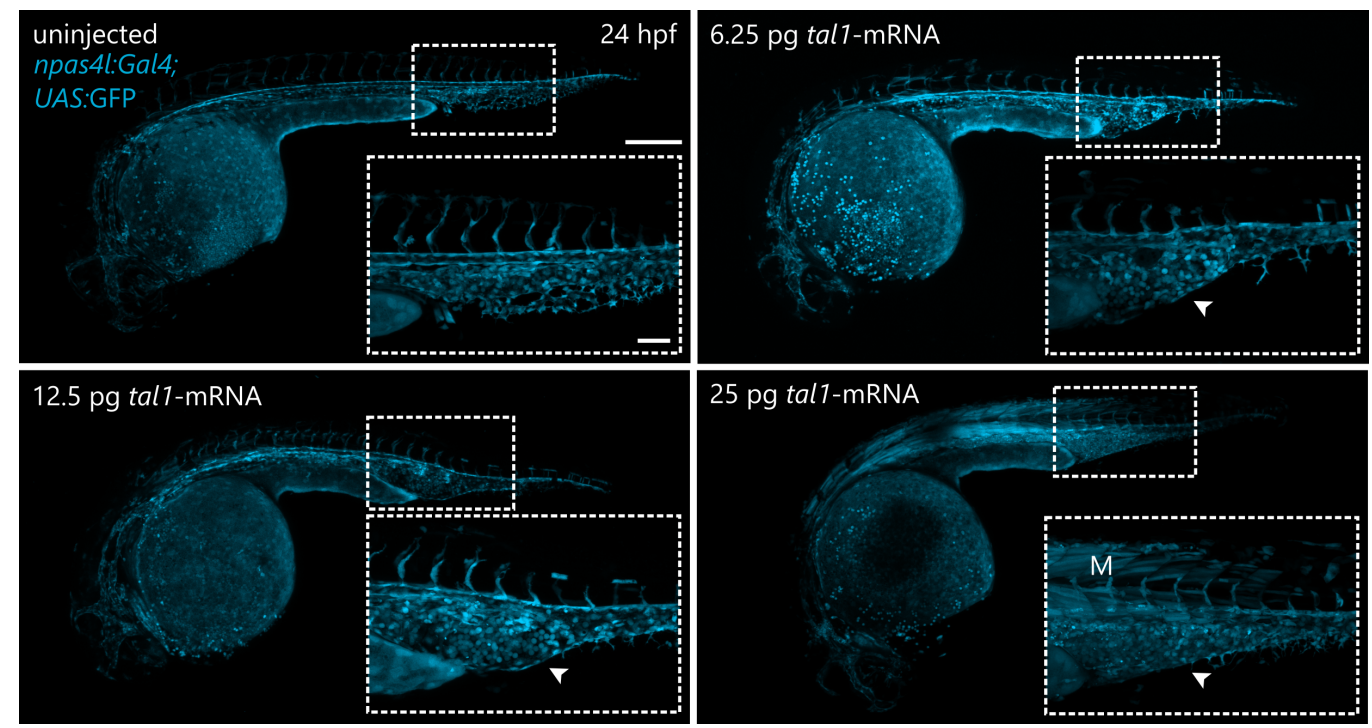

Figure 4.38: Injections of tal1 mRNA into wild-type embryos leads to an increase in blood cells. Titration of optimal dosing for the rescue experiments. A dose dependent increase of circulating blood cells (arrowheads) upon treatment with tal1 mRNA as well as ectopic npas 4 l reporter expression in skeletal muscle (M) at $25 \mathrm{pg}$ injections was observed. Scale bars: $200 \mu \mathrm{m}$ (overview), $50 \mu \mathrm{m}$ (closeup). 
regulatory mechanism such as a negative feedback loop between those genes.

Given the strong rescue achieved with tal1 mRNA overexpression, it is tempting to consider trying to rescue npas $4 \mathrm{l}$ mutants to adulthood. However, global overexpression of the tal1 causes defects that must be overcome in order to achieve this. The overexpression of tal1 in wild-type fish used to optimized the dosing showed a strong increase in blood present in the embryo as early as $24 \mathrm{hpf}$ (Figure 4.38). In high doses such as $25 \mathrm{pg}$, even ectopic npas $4 l$ expression in skeletal muscle could be observed. On top of that, the failure to develop a brain vasculature is likely to burden the fish and an attempt to raise rescued mutants has, therefore, not yet been made as an official animal proposal would be necessary.

\subsection{7 npas4l-mRNA injection enhances endo- thelial transplantation efficiency}

An alternative strategy to rescue the npas $4 l$ mutant phenotype is the transplantation of wild-type cells that can form functional endothelial and blood cells in npas4l mutant embryos. However, endothelial transplants are technically challenging, inefficient and often do not reach the level of mosaicism in transplanted embryos required for a functional rescue of an endothelial phenotype.

As npas4l-mRNA overexpression induces an endothelial cell fate ectopically (Reischauer et al., 2016), I tried to boost endothelial transplant efficiency by injecting npas4l-mRNA into donor embryos and transplanting them into wild-type hosts (Figure 4.39A) to see whether more endothelial cells can be observed after this treatment. As an endothelial marker for this experiment, I chose a fli1a transgenic reporter line as this gene is not directly induced through the downstream signaling of Npas4l and therefore provides a better readout for an endothelial cell fate compared to markers like $k d r l$.

The data suggest that npas $4 \mathrm{l}$ mRNA injection can indeed lead to a higher transplantation efficiency with almost all transplanted embryos showing transplanted endothelial cells (Figure 4.39B). Even some endocardial transplants have been obtained which is an enormous technical challenge in the field. Perhaps even more interesting yet was an unexpected side observation: Cells in uninjected donors as visualized by nls-mTagBFP2 fluorescence committed to all kinds of tissues and formed a salt-and-pepper pattern throughout the 
embryo. Some of these cells were also endothelial cells (Figure 4.39C). In contrast to that, the npas4l-mRNA injected donor cells were mainly detected in areas that form endothelial cells and co-expressed endothelial markers.
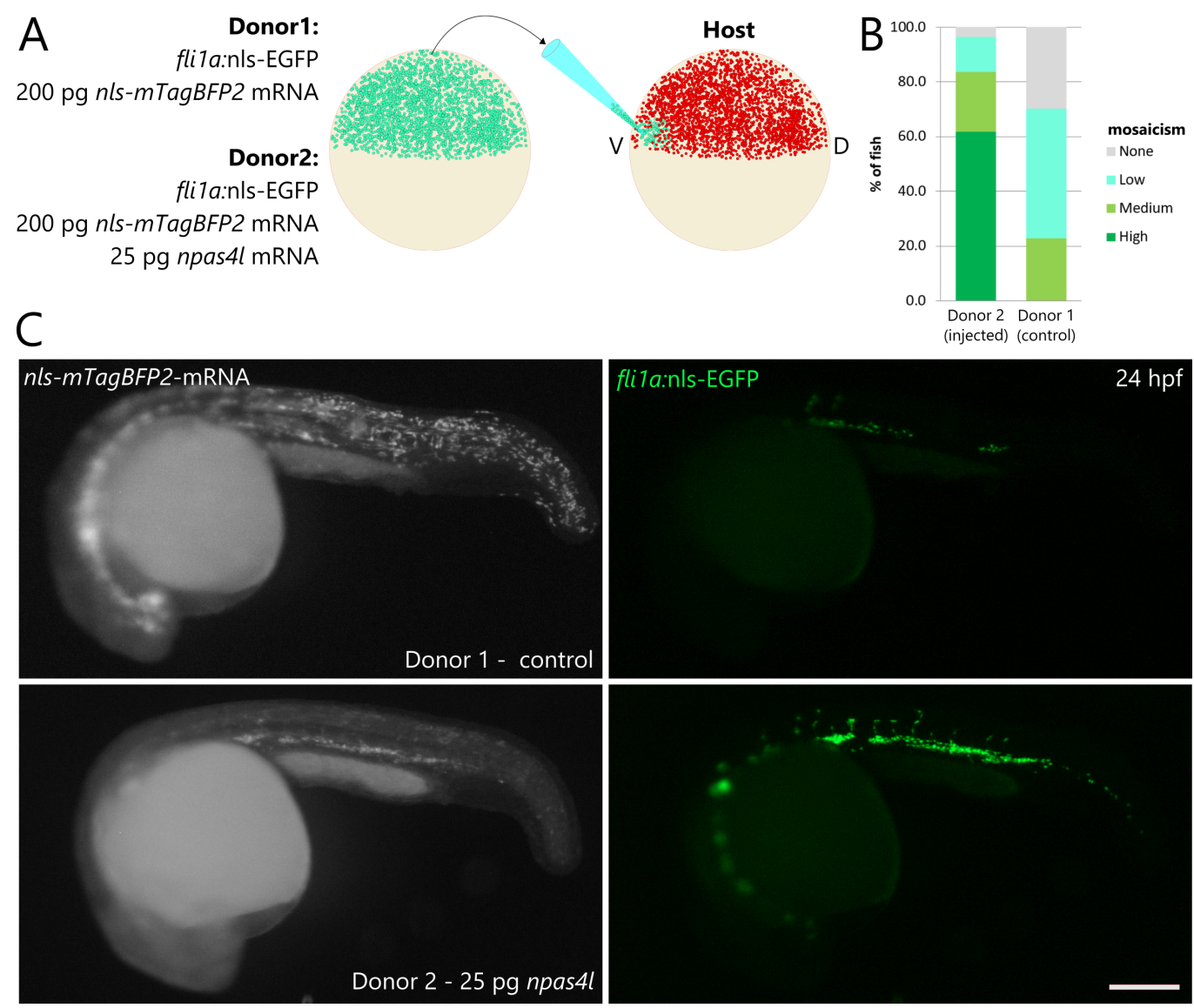

Figure 4.39: Endothelial transplant efficiency can be improved by mRNA overexpression of npas $4 \boldsymbol{l}$ in donor embryos. (A) Schematic representation of the experiment. Cells from control donors and npas 4 -mRNA injected embryos were transplanted into wild-type hosts. mTagBFP2-mRNA was injected as well to detect and trace all transplanted cells. (B) Semi-quantitative analysis of fli1 a mosaicism derived from host cells in npas 4l-mRNA injected and control embryos. (C) Imaging of representative embryos with high number of fli1 $a$ expressing donor cells in both conditions. Scalebar: $200 \mu \mathrm{m}$. I thank Hans-Martin Maischein for performing the transplants shown in the panel and for teaching me how to transplant myself.

I wanted to know whether the lack of cell types other than endothelial cells in the npas $4 l$ injected group is due to apoptosis of cells from other lineages or whether the npas 4 l-mRNA overexpression forces an endothelial cell fate in all transplanted cells. Therefore, I transplanted cells into the animal pole of the embryo to see whether transplants into this area of the gastrula, that 
is normally incompetent of producing endothelial cells, can give rise to these cells if they have been previously primed by npas $4 \mathrm{l}$ mRNA overexpression. Although this experiment has not been repeated yet and only been carried out using a low number of embryos, the data suggest that npas $4 l$ injected cells transplanted on the animal pole may still give rise to high numbers of fli1a positive endothelial cells (data not shown). It is therefore likely that npas $4 \mathrm{l}$ overexpression causes an endothelial cell fate independent of the part of the gastrula it is transplanted in. 



\section{Chapter 5}

\section{Discussion}

\section{1 npas4l-V5 $5^{\text {bns314 }}$ enables Npas4l protein de- tection and retains Npas4l function}

To allow the detection of Npas4l despite the lack of available antibodies for this protein, I designed and generated a knock-in allele of npas $4 l$ encoding a C-terminal V5 tag on the endogenous peptide sequence. The allele complements known mutant alleles, and homozygous carriers of bns314 survive to adulthood and show a normal life span indicating that the tagged Npas4l-V5 protein retained Npas4l function.

Using wholemount immunohistochemistry, I was able to detect a weak V5-signal in the area of the lateral plate mesoderm from the 2 somite stage to the 10 somite stage. A detection of the tagged proteins in Western blot was not successful. This is likely due to low protein abundance. Predictions of sequence motifs promoting the proteasomal degradation of the protein suggest that this might be due to a strong degradation sequence (PEST).

Such an effect could be overcome by a protein pull-down with a high amount of protein as input material. If the protein was detectable in a Western blot after pull-down, this would enable analyses through mass spectrometry of a purified Npas4l transcription factor complex, thereby shedding light on its possible interaction partners.

Although such proteomic analyses would reveal valuable insights, I decided to postpone this part of the project after the initial validation of the tools mentioned above due to time constraints and because this project is likely to require iterative trials and optimization. Now that some other projects have 
been concluded, it has once more become of the top priority projects and a definite short-term goal (compare to section 5.6.5).

\subsection{The npas $4 l^{\text {bns313 }}$ allele labels npas4l pos- itive cells in vivo in embryos and adults}

To visualize npas4l expression in vivo, I generated a fluorescent reporter line by knocking in a Gal4 driver into the endogenous npas 4 l locus. I was able to detect this reporter from early somite stages onwards and even in adults.

The expression at early stages in endothelial cells, yolk, circulating cells, and cells in the pectoral tail fin as well as both cardiac and skeletal muscle looks similar to the expression of the lateral plate mesoderm marker $d r l$ (Prummel et al. 2019; Figure 1.2). Although the expression of endothelial reporters in paraxial mesoderm-derived muscle tissue is generally rejected as potential off-target fluorescence, there is mounting evidence of paraxial mesoderm-derived endothelial cells (compare section 1.2.1). The discovery of bipotent cells in the zebrafish paraxial mesoderm that can give rise to specialized hematopoietic stem cells (Nguyen et al., 2014; Sahai-Hernandez et al., 2020) as well as the differentiation of etsrp reporter positive endothelial progenitors into skeletal muscle observed in etsrp mutants add plausibility to the observed patterns. In addition to this indirect evidence, McGarvey et al. (2020) recently published a preprint showing tissue specific enhancers through single cell ATACseq in the promoter region of npas 4 l. Not only did thay find an apparently endothelial specific enhancer candidate but also two candidates that are open in a number of different other tissues. Preliminary tests of these enhancers in vivo appear to confirm these predictions and will be included in the reviewed version of their manuscript (data not shown). Also, I have observed npas 4 l expression in skeletal muscle cells by single cell RNAseq independently of npas $4 l$ reporter expression.

npas $4 \mathrm{l}$ is known to be transiently expressed during early endothelial development, but whether it is expressed in adult tissues was not known. I did not see adult endothelial expression. However, the reporter signal in cardiomyocytes and some cells in the fin was still observed. Several distinct areas in the brain also showed reporter fluorescence. However, as NPAS is short for "neuronal PAS domain containing transcription factor" and other Npas transcription factors are predominantly expressed in neurons (Massari and Murre, 2000), these results were not entirely implausible. 


\subsection{The npas 4 l reporter is reactivated during regenerative processes}

When I tested for npas 4 l reporter expression in the fin and, therefore, amputated the fin tip to analyze it by confocal microscopy, only few apparent osteoblast cells showed reporter expression. Hypothetically, this could be due to the differentiated state of these endothelial cells and npas4l might be re-expressed in endothelial cells during regenerative processes like the fin tip amputation. Therefore, I collected a sample of the regenerating fin again 3 days post amputation.

Against expectations, I did not observe any endothelial expression but instead detected a strong induction of reporter expression in the blastema tissue, which consists of dedifferentiated cells forming the regenerate. It will be interesting to investigate whether npas $4 l$ is expressed in these cells only or whether it serves a physiological function during regeneration. A collaborative project investigating this is in progress in collaboration with Srinivas Allanki.

\subsection{The npas $4 l$ mutant phenotype is caused by multiple defects phenocopied by dif- ferent downstream effectors}

\subsection{1 npas $4 l$ reporter positive cells in $n$ pas $4 l$ mutants do not migrate or differentiate and instead con- tribute to paraxial and intermediate mesoderm- derived tissues}

The absence of endothelium and blood in npas4l mutants has been reported before (Stainier et al., 1995; Reischauer et al., 2016; Marass et al., 2019), but little was known about what happens to npas 4 l positive cells in the absence of Npas4l function. Schoenebeck et al. (2007) reported an increase in cardiomyocyte numbers in cloche/npas 4 l mutants. However, whether these additional cardiomyocytes derived from npas $4 l$ positive cells was not tested due to a lack of appropriate tools.

To investigate the npas $4 l$ positive lineage in npas $4 l$ mutants, I induced an in-frame deletion on the reporter allele affecting the region encoding the 
binding site with the goal to retain reporter function but abolish Npas4l function. As shown by complementation tests this strategy was successful and did not alter the expression pattern in npas $4 l^{\text {bns } 423}$ heterozygous animals as determined by comparison to carriers of the unmutated npas $4 l^{\text {bns } 313}$ allele.

When analyzing the npas 4 l reporter positive lineage in npas $4 l$ mutants focusing on the endothelial progenitor cells localized in the trunk region, I observed a migration and differentiation failure of npas $4 l$ reporter positive cells. Instead, these cells contributed to the intermediate mesoderm-derived pronephric ducts and the paraxial mesoderm-derived skeletal muscle. Examination of single cell ATACseq data in whole npas 4 l embryos (McGarvey et al., 2020) suggest that both the pronephric tubule and the skeletal muscle populations are enlarged in npas $4 l$ mutants and might, therefore, represent a global lineage differentiation defect.

A paraxial mesoderm contribution similar to the one observed in npas $4 \mathrm{l}$ mutants was recently reported by Chestnut et al. (2020). In an approach similar to ours, the authors used a reporter line to follow etsrp reporter positive cells in etsrp mutants similar to our approach (Chestnut and Sumanas, 2020) and observed a contribution of endothelial progenitors to skeletal muscle but no pronephric tubule contribution. As etsrp is one of the three major transcriptional effectors downstream of Npas4l (Marass et al., 2019), I hypothesized that tal1 and lmo2 may be responsible for the effects seen observed in npas 4 l, but not etsrp mutants - the migration defect and contribution to the pronephric tubules. To analyze this, I generated mutants for all the mentioned genes and compared their phenotypes to the phenotype observed in npas4l mutants.

\subsubsection{Tal1 and Lmo2 suppress an intermediate meso- derm fate}

In tal1 mutants, the npas $4 l$ reporter expressing cells fail to migrate towards the midline and, thus, to contribute to the vascular cord. Instead, these cells remain in a ventrolateral position and contribute to the pronephric tubules. This phenotype is not observed in etsrp mutants. However, endothelial lineage specification defects leading to an increase in the number of pronephric tubule cells have been previously observed in hand2 mutants (Perens et al., 2016) and in tbx16 mutants (Rohde et al., 2004). Both hand2 and tbx16 mutants display a reduction in tal1 expression (Rohde et al., 2004; Warga et al., 2013; Perens et al., 2016). Moreover, overexpression of either tal1 
(Gering et al., 2003) or hand2 (Perens et al., 2016) caused a reduction in the size of the pronephric tubules and an increase in the number of endothelial cells. Conversely, the loss of the osr 1 transcription factor gene leads to an increase in endothelial progenitors in zebrafish at the expense of kidney progenitors (Mudumana et al., 2008). In mouse Osr1 mutants, several key regulators of kidney development fail to be expressed and the metanephric mesenchyme appears to undergo apoptosis. Examining endothelial progenitors in the presumptive kidney region in these animals (Wang et al., 2005) will be informative. Moreover, the presence of endothelial cells in human kidney organoids (Takasato and Little, 2016) suggests that they may arise from in vitro differentiated intermediate mesoderm. Altogether, these data indicate that Tal1 — like Hand2, Osr1 and Tbx16 - plays a role in cell fate decision between intermediate mesoderm-derived lineages and endothelial/blood cells by promoting endothelial and hematopoietic cell fates. It will be interesting to investigate whether the bHLH transcription factors Tal1 and Hand2 physically interact in this cell fate decision.

Tal1 and Lmo2 interact physically (El Omari et al., 2011) and function synergistically (Gering et al., 2003) in transcription factor complexes. $\mathrm{Mu}-$ tants in these genes thus share many phenotypic aspects. However, although tal1 and lmo2 mutants lack red blood cells, most endothelial progenitors in lmo2 mutants reach the midline while many endothelial cells in the PCV are Pax2a positive, indicating a lineage specification but not a migration defect. From these results, it appears that endothelial progenitor migration depends on Tal1 but not on Lmo2. It remains possible, however, that $l m o 2^{b n s 499}$ is a hypomorphic allele.

\subsubsection{Etsrp suppresses a paraxial mesoderm fate}

In contrast to the phenotypes in tal1 and lmo2 mutant embryos, endothelial progenitors migrate to the midline in etsrp mutants but fail to acquire endothelial characteristics and form distinct vessels. Also, there is an increase in npas 4 l reporter expressing skeletal muscle cells in etsrp mutants, suggesting a specification defect of endothelial progenitors. Both phenotypes are similar to what has been described by Chestnut et al. (2020) using a reporter-based strategy to determine the fate of etsrp reporter expressing cells in etsrp mutants. However, my data do not support the conclusion of Chestnut et al. (2020) that tal1 mutants phenocopy etsrp mutants nor do they indicate that tal1 acts downstream of Etsrp. Indeed, the ability to visualize npas4l reporter expressing cells in tal1 and etsrp mutants clearly reveals their phenotypic differences (schematic model in figure 5.1). 
The paraxial mesoderm has been suggested to be an additional source of endothelial cells (Couly et al., 1995; Wasteson et al., 2008; Nguyen et al., 2014; Stone and Stainier, 2019; Pak et al., 2020). In zebrafish, the paraxial mesoderm has been reported to be a source of a bipotent cell population that can give rise to hematopoietic stem and progenitor cells or muscle progenitors (Nguyen et al., 2014; Sahai-Hernandez et al., 2020). Also, ectopic etsrp overexpression has been reported to trigger endothelial marker expression and behavior in cells of the paraxial mesoderm (Veldman et al., 2013). A close relationship between endothelial cells and paraxial mesoderm is, therefore, plausible.
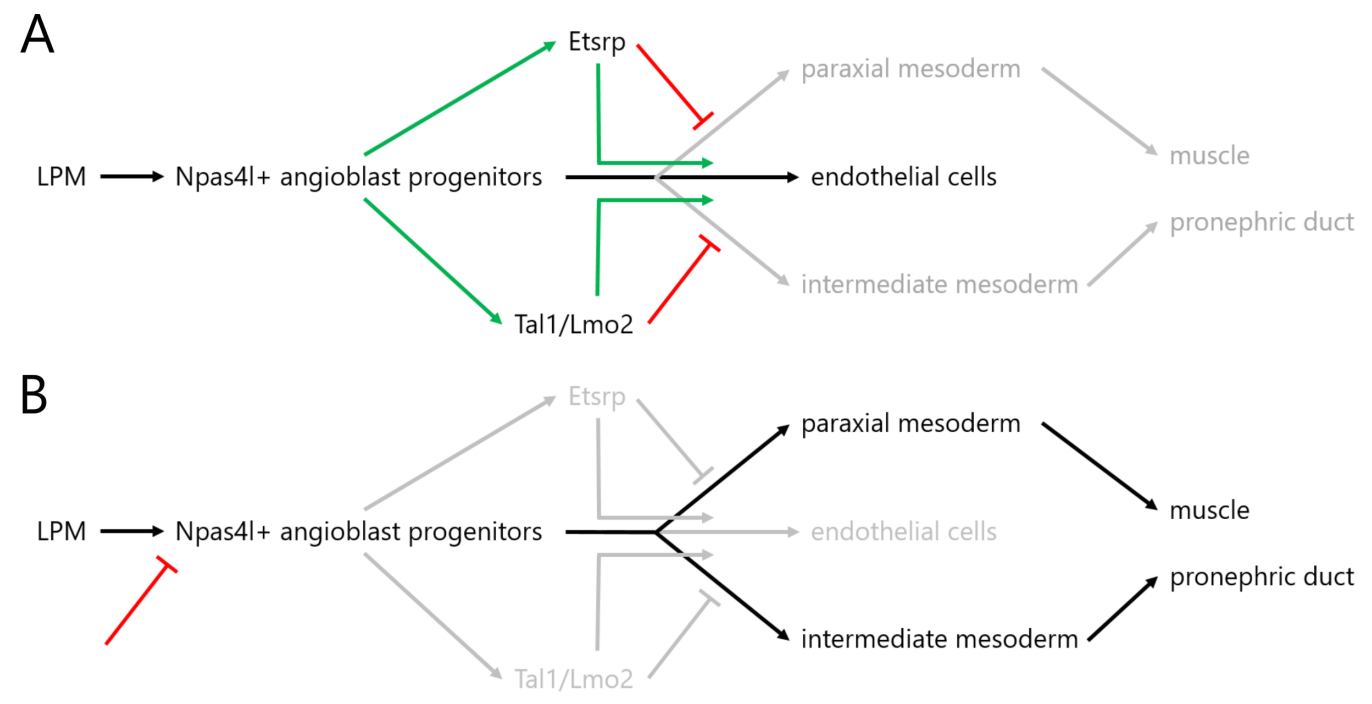

Figure 5.1: Schematic model of Npas4l function during endothelial specification. (A) In wild-type embryos, Npas4l in the lateral plate mesoderm (LPM) drives the expression of etsrp, tal1 and lmo2, leading to the expression of endothelial markers and repressing alternative lineages in angioblast progenitors. (B) In the absence of Npas4l, etsrp, tal1 and lmo2 are not expressed, leading to the commitment of endothelial progenitors to the paraxial mesoderm-derived muscle through lack of Etsrp and intermediate mesoderm-derived pronephric tubules through the lack of Tal1 and Lmo2.

\subsection{Endothelial cells in zebrafish cannot form in absence of both Tal1 and Etsrp func- tion}

Although npas $4 l$ mutants lack most endothelial cells, a few cells in the tail display endothelial characteristics (Stainier et al., 1995; Reischauer et al., 
2016). Against expectation, an even stronger endothelial defect characterized by the complete loss of endothelial cells was observed in tal1//- etsrp $^{-/-}$ embryos, indicating that no embryonic endothelial cells can develop independently of Tal1 and Etsrp function.

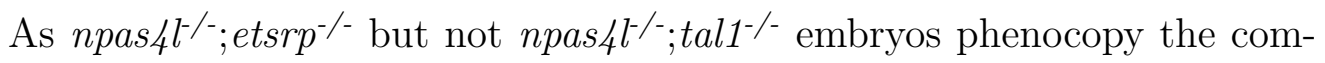
plete lack of endothelium, I hypothesize that the remaining endothelial cells in npas $4 \mathrm{l}$ mutants express etsrp but not tal1 independently of Npas4l function. Notably, endothelial cells in npas $4 l$ mutants always develop in the tail region, mostly posterior to the pronephric tubules. Thus, in the absence of Npas4l function, endothelial cells may specify only in areas where they are unable to contribute to pronephric tubules and it will be important to understand how etsrp is induced in these cells. An experimental idea to approach this issue will be presented in section 5.6.6.

\subsection{Future perspectives}

\subsubsection{What is the earliest defect in npas 4 l mutants and when can it be observed for the first time?}

The discovery that npas $4 l$ reporter positive cells in npas $4 l$ and tal1 mutants do not migrate towards the midline and contribute to pronephric tubules raises the question how these two phenotypes are related and whether they depend on each other. Three scenarios appear to be plausible at this time:

1. npas 4 l reporter positive cells contribute to the pronephric ducts and therefore fail to migrate,

2. npas $4 l$ reporter positive cells fail to migrate, remain lateral and consequently contribute to the pronephric ducts, or

3. the two phenotypes are are independent from each other.

In favor of the first model, Perens et al. (2016) showed that the effect of hand2 overexpression on cell fate decisions between endothelium and pronephric tubule cell lineage only works in early somite stages and is no longer effective at the 10 somite stage when endothelial migration starts in the embryo. These data suggest that lineage decisions between endothelial and pronephric tubule cells are made at stages prior to endothelial migration. However, some endothelial progenitors in npas4l, tal1 and especially lmo2 mutants express both pronephric tubule and endothelial markers but manage to migrate towards the midline. 
To investigate this phenotype, I plan to analyze immunostainings of npas $4 \mathrm{l}$ and tal1 mutant embryos at stages prior to angioblast migration. By staining them for the npas4l reporter, Fli1a and Pax2a. If mainly cells that are positive for the npas4l reporter and Pax2a, but negative for Fli1a will be detected, it would suggest that the contribution to the pronephric tubules is determined in very early stages prior to the migration to the midline. If mainly cells positive for the npas4l reporter and Fli1a, but negative for Pax2a will be detected, Pax2a would be a marker expressed later in these cells, raising the possibility that the failure to migrate precedes the contribution to the pronephric tubules. If all three markers will be present in early stages, migration and lineage decisions may be independent. More specialized experiments would have to be designed to investigate this question in this case.

\subsubsection{A platform for the systematic testing of early transcription factors during vasculogenesis}

The re-analysis of well known mutant phenotypes using my newly generated reporter lines allowed me to discover new phenotypes in npas4l, tal1 and lmo2 mutants. The reason why we found these new phenotypes is that the npas $4 l$ reporter labels endothelial progenitors even if they do not express any later markers of endothelial cells, including widely used markers such as fli1a and etsrp, and allows the tracing of these cells through development. This is especially helpful to uncover alternative lineage decisions in endothelial progenitor cells. Such phenotypes could not be analyzed with previously published tools. Going through the literature, I found many more candidates of early endothelial transcription factor genes in which new phenotypes may be discovered (compare to section 1.3.5). One of the candidates, that I am already growing for this purpose in collaboration with Sébastien Gauvrit, is a null allele for hhex. These mutants are ready to be crossed and analyzed in the npas $4 l$ reporter background.

I suggest to apply a standard panel of tests to the investigation of early endothelial genes. The workflow would be quite simple:

1. Analyze the loss of function phenotype of the candidate gene in the npas $4 \mathrm{l}$ reporter background. Ideally, a mutant should be generated, but morphants or crispants could also be used to allow for faster screening.

2. Overexpress the candidate gene in npas4l reporter positive embryos. 
3. Pay special attention to changes in lateral plate mesoderm-derived tissues or tissues of other mesodermal lineages (compare to Figure 1.2).

This strategy would not only allow the discovery of potential new endothelial phenotypes but also give insight into the earliest steps of endothelial development and the cell fate decisions involved in endothelial specification. Additionally, standardized tests including standardized imaging and stages would provide the best comparative insight into the transcriptional network regulating early endothelial development that has ever been obtained.

The standardization of testing procedures would also have the benefit that the tools and instructions could be sent to other labs to test their mutants for early endothelial defects. This way, the data collected for new mutants or morphants of early endothelial genes may be collected in a way that generates comparable data and aid the immediated placement of new factors in the broader context of early endothelial development.

\subsection{3 npas 4 l function during heart development}

Due to time constraints I focused entirely on cell fate decisions during early vasculogenesis in the trunk region of the embryo. However, the npas4l reporter appears to label the first heart field at early stages (data not shown) and would allow a screening for alternative cell fates of the cardiac mesoderm in mutants of npas4l, etsrp, tal1 and lmo2. Both npas4l and etsrp mutants have an increased number of cardiomyocytes (Schoenebeck et al., 2007; Palencia-Desai et al., 2011), but whether they or tal1 mutants may show additional cell fate decision defects is yet unknown.

Since the npas $4 l$ reporter line is already part of the background of npas 4 , etsrp, tal1 and lmo2 mutants, a preliminary analysis of these phenotypes would not require a lot of time. Due to its simplicity and short time frame this project could be given to a student and completed within a Bachelor or Master thesis.

\subsubsection{Does tal1 overexpression in low doses cause so- mite defects?}

A somite defect in wild-type embryos after overexpression of tal1 as determined by in situ hybridization for MyoD in early stages has been reported in several studies (Gering et al., 1998, 2003; Dooley et al., 2005). I did not 
observe any obvious somite defects after tal1 injection but did also not analyze them in detail using specific markers. As ectopic overexpression can cause off-target defects, it is possible that defects observed in previous studies were caused by delays or off-target effects. However, it would be important to investigate whether this phenotype can be observed in the rescues done in this study. As tal1 mutants did not show an increase in npas $4 l$ reporter positive skeletal muscle cells like the phenotype seen in etsrp mutants, the injections of tal1 mRNA may have a cell non-autonomous effect on the skeletal muscle. However, my injections of tal1 mRNA into wild-type embryos also showed that in relatively high doses even ectopic expression of npas $4 l$ could be induced by tal1 mRNA. As the highest doses tested in this thesis (25 pg) are still four times lower than tal1 mRNA doses injected into wild-type embryos in other published studies, an increase in off-target effects would be a plausible explanation.

However, it is still important to address such issues to be able to explain differences between my data and previously published tal1 overexpression data. Therefore, I suggest comparing uninjected embryos to those having been injected with $5 \mathrm{pg}$ and $100 \mathrm{pg}$ tal1 mRNA into wild-type embryos and to do wholemount in situ hybridization for myod in these embryos at early somite stages. This experiment will be a fast and easy way to test whether rescues with low doses of tal1 cause somite defects similar to observations reported after injections of larger doses (Gering et al., 1998, 2003; Dooley et al., 2005).

\subsubsection{Direct interaction partners of Npas4l}

The direct interaction partners of Npas4l are not yet known. It is likely that Type2 bHLH transcription factors are amongst the proteins interacting directly with Npas4l, but the complex may be more complicated than forming a simple heterodimer. Knowing the binding partners of Npas4l would help to understand how its transcriptional activity is regulated.

I discussed the npas 4 l- $V 5$ allele that I generated before (see section 5.1). I have not yet had the time to perform the necessary experiments and to pull down the transcription factor complex for proteomic analyses, but this would be one approach to get more mechanistic insight into this transcription factor. 
There is a parallel approach, however, that could be pursued. As a bHLHPAS transcription factor, Npas4l is known to form heterodimers with aryl hydrocarbon receptor nuclear translocator (AHR/ARNT) proteins (Bersten et al., 2013). There are five of these genes in zebrafish: arnt, arnt2, arntl1a, arntl1b and arntl2. Mutants for the factor that interacts with Npas4l should show a cloche/npas4l mutant phenotype as type 1 bHLH-PAS transcription factors like Npas4l cannot show transcriptional activity without an AHR/ARNT protein.

arnt and arnt2 mutants have been reported before, but their mutations do not appear to cause strong vascular defects (Marchi et al., 2020). arntl1a morphants show some ISV sprouting defects (Jensen et al., 2012). No vascular defects in arntl1b mutants have been reported (Wang et al., 2015). It may be interesting to combine different published and newly generated mutants to test which combinations may lead to a phenotype similar to the one observed in npas 4 l mutants.

\subsubsection{How is etsrp expressed in the remaining endothe- lium of npas $4 l$ mutants?}

Through the generation of npas4l/etsrp double mutants, I determined that the remaining endothelial cells present in npas 4 l mutants depend on etsrp. But how is this gene expressed without transcriptional activity of Npas4l? In this section, I argue that good candidates for being direct upstream regulators of etsrp expression in the remaining endothelium of npas4l mutants may be the F-box transcription factors Foxc1a and Foxc1b.

The etsrp promoter region has been reported to be bound by the two F-Box transcription factors Foxc1a and Foxc1b through electrophoretic mobility shift assay (EMSA) and chromatin immunoprecipitation PCR (ChIPPCR) (Veldman and Lin, 2012). Morpholino knock-down of both foxc1a and foxc1b reduces etsrp expression and causes strong vascular defects (De Val et al., 2008; Veldman and Lin, 2012). I therefore hypothesize that these two transcription factors are responsible for the etsrp expression required to induce endothelial cell specification in the tail of npas $4 l$ mutants. To test the plausibility of this hypothesis, I checked the regulation of both genes in RNAseq profile of fli1a reporter positive cells in npas $4 l$ mutants and the single cell RNAseq data of npas 4 l reporter positive cells. In fli1a reporter positive endothelial cells of npas 4 l mutants, both genes are highly upregulated, whereas in the single cell RNAseq data, the highest expression can 
be seen in etsrp and fli1a expressing cells of the npas 4 l mutant. Furthermore, especially foxc1b is expressed in almost the same population of cells as npas $4 \mathrm{l}$, both in heterozygotes and npas $4 \mathrm{l}$ mutants. Together, these data suggest that the remaining fli1a positive endothelial cells in the npas $4 \mathrm{l} \mathrm{mu-}$ tant show high levels of foxc1a and foxc1b. These factors may activate etsrp in the remaining endothelial cells of npas $4 l$ mutants.

TALEN mutants for foxc1a show cardiac and somite defects through direct transcriptional effects on nkx2.5 (Yue et al., 2018) and aldh1a2 (Li et al., 2015) expression as well as angiogenic defects (Jiang et al., 2018). However, early endothelial and hematopoietic genes like etsrp and tal1 are also downregulated in those mutants (Yue et al., 2018), suggesting that an earlier endothelial defect could be present, although no morphological defects were reported in early stages. The vascular defects as well as other phenotypes observed are stronger in foxc1a/foxc1b double mutants, suggesting synergistic effects between different double mutants (Xu et al., 2018; Jiang et al., 2018). My suggestion is to inject foxc1a and foxc1b morpholinos into npas 4 l mutants to test whether this treatment reduces the number of endothelial cells in their tails. If this is the case, I suggest generating the foxc1b mutant and generate or import a mutant for foxc1a.

\subsubsection{An upstream candidate of $n p a s 4 l$}

In the previous section, I argued that foxc1a and foxc1b are potentially activating etsrp in the remaining endothelium of npas $4 \mathrm{l}$ mutants. However, it will be important to analyze whether the foxc1a/foxc1b knockout or knockdown may also regulate npas $4 l$ expression. In foxc1a mutants, expression of the Npas4l downstream targets etsrp and tal1 is down-regulated. In addition, both foxc1a and foxc1b are already expressed during gastrulation and not regulated in expression profiles of early (1 and 6 somite stage) npas $4 l$ mutants or after overexpression of npas $4 l$ during gastrulation (based on data from Marass et al., 2019), suggesting that they either work in parallel or may even function upstream of npas4l.

If Foxc1a and Foxc1b regulate npas 4 l expression, they are likely to bind to the npas $4 l$ promoter. The mammalian FOXC1 recognition sequence is well known (Khan et al., 2018) and might be conserved for zebrafish Foxc1a and Foxc1b. Therefore, I searched for an annotated FOXC1 recognition sequence in the enhancers of npas $4 l$ that have been recently identified by single cell ATACseq (McGarvey et al., 2020) and found a predicted recognition site in 
enhancer $2 \mathrm{~b}$. Conclusively, foxc1a and foxc $1 \mathrm{~b}$ represent the best candidates for regulation of npas4l that I am aware of at the moment and I strongly suggest to look into these candidates. As foxc1a/foxc1b double mutants do not display a phenotype that is as severe as the npas $4 l$ mutant phenotype (Jiang et al., 2018), it cannot be expected that these genes represent the only factors involved in the promotion of npas $4 l$ expression, but as no upstream regulators of npas $4 l$ are known so far, it would nevertheless be a very interesting finding.

\subsubsection{The evolution of early endothelial transcription factors}

An endothelial cell layer is a unique feature shared by all vertebrates. Although all vertebrate model organisms share a set of relatively conserved endothelial effectors such as cdh5 and the VEGF-signaling pathways, the transcriptional network driving early endothelial specification shows many species-specific differences (compare to section 1.1).

Exploring how the transcriptional network inducing the specification and differentiation of endothelial cells evolved in vertebrates will give valuable insights into the endothelial cell identity and specification. In addition to computational analyses, a direct comparison of multiple endothelial transcription factors would help reveal both similarities and and differences in function. The approach outlined in section 5.6.2, for instance, could help to generate comparable data that can feed back into the computational predictions of the evolution of factors required for endothelial specification.

\subsection{9 npas4l reporter expression in species that lack endothelium or npas 4 l}

McGarvey et al. (2020) recently published a preprint of single cell ATACseq and identified putative enhancers upstream of npas4l. Among them was one candidate that appeared to be specific to endothelial cells and blood. I confirmed this expression pattern by generating a transgenic line (data not shown as this is part of a collaborative experiment that will be included in the updated version of (McGarvey et al., 2020)). As this short enhancer now allows the generation of npas $4 \mathrm{l}$ transgenic lines, I pondered generating npas $4 l$ reporter mice. 
Although there are often functional differences, the expression of most early transcription factors involved in lateral plate mesoderm development like BMP, Eomesodermin and Brachyury are quite conserved (Prummel et al., 2019). npas $4 l$ is not present in mammals (Ingo Ebersberger, personal communication), but it is possible that the upstream regulatory mechanism inducing npas $4 \mathrm{l}$ expression is conserved and regulates other factors. If a transgenic line with the endothelial enhancer of npas $4 l$ was to still show an endothelial expression in mice, such observations would suggest that the factor inducing endothelial specification in mice may share common upstream regulators with zebrafish npas4l. This would give insight into how the transcriptional network evolved that is required to induce the specification of endothelial cells in vertebrates.

Moreover, it would be informative to see if and where such an enhancer (or the endogenous npas $4 l$ l) would be expressed in chordates like Amphioxus (Branchiostoma). These animals do not have endothelial cells lining their vessels but express the transcription factor gene npas4l. These species are very closely related to the common ancestor of endothelial cell possessing vertebrates and the transcriptional network required for the induction of endothelial cells is already largely in place. To see where npas4l would be expressed in these animals will give insight into the evolutionary ancestry of endothelial cells. Would it be expressed in paraxial and/or intermediate mesoderm? Or would it otherwise be expressed in the amoeboid cells in their vascular system suggesting that the endothelial cells might have developed from this ancient cell type which is present even in invertebrate species?

\subsubsection{Rescuing npas4l mutants to adulthood}

Global injections with tal1-mRNA rescued the vasculogenesis defects in npas $4 \mathrm{l}$ mutants. This effect could potentially be used to raise npas $4 l$ mutants to adulthood. This could be used to study potential functions of Npas4l in adult stages such as regenerative processes. However, the brain vasculature did not form in tal1 mRNA injected embryos, probably because large brain vessels develop through a specialized angiogenic mechanism and not through vasculogenesis (Proulx et al., 2010). Additionally, most embryos developed a pericardial edema in tal1-mRNA injected embryos. As Tal1 is a powerful transcription factor, these effects might be caused by the ectopic expression of tal1. A transient and tissue specific expression of tal1 in early hematovascular progenitor cells may be sufficient to rescue npas $4 l$ mutants to adulthood. 
In the previous section, I mentioned the availability of a new endothelial specific enhancer in the promoter region of npas 4 l. Unlike other endothelial promoter elements, npas $4 l$ enhancers should still be expressed in the npas 4 l mutant, making it the ideal tool to attempt a functional rescue. A floxed tal1 overexpression construct under the npas $4 l$ endothelial enhancer could theoretically be used to rescue Npas4l function through tissue-specific expression of the transgene in npas $4 \mathrm{l}$ mutants during early stages. This transgene could then be floxed out at a later stage to analyze which effects Npas4l has during late larval and adult stages.

\subsubsection{Can npas4l overexpression be harnessed as a tool to improve endothelial transplantation ef- ficiency?}

npas $4 \mathrm{l}$ mRNA overexpression increased the endothelial transplant efficiency and even appeared to lead to an endothelial cell fate when transplanted into the animal pole of the embryo. During normal embryonic development, only cells around the margin of the gastrula give rise to endothelial cells (Vogeli et al., 2006), suggesting this to be an effect that influences the fatemap of the embryo. A similar behavior has been seen for sox17-overexpression in donor cells which produces ectopic endodermal cells and can be used to rescue endoderm in sox17/cas mutants, which lack endoderm (Kikuchi et al., 2001).

Although the data I collected on this project is preliminary, it will be worthwhile to develop a standardized protocol based on transplanting npas4lmRNA injected donor cells and thereby increasing the efficiency of endothelial and especially the more challenging endocardial transplants. This would not only be a great tool for the zebrafish community researching the vascular system but could also provide insights into the mechanisms that induce an endothelial cell fate in zebrafish through the transcriptional activity of Npas4l.

\subsubsection{Miscellaneous experiments}

Above, I outlined what I think to be the most intriguing project ideas and directions. However, there are a number of short experiments that do not quite qualify as a project but could address some fundamental biological concepts and questions. 


\subsubsection{Do npas4l mutants have an increased number of $n$ pas 4 l reporter positive myocardial cells?}

Schoenebeck et al. (2007) reported that npas 4 l mutants have an increased number of myl'7 positive myocardial cells. However, whether this effect is cell autonomous and caused by the contribution of npas $4 l$ reporter positive cells to myocardial precursors is not known. Therefore, I crossed the npas $4 \mathrm{l}$ mutant reporter into a nuclear myl't-transgenic background and am going to use these fish to count npas $4 \mathrm{l}$ reporter positive cardiomyocytes at $48 \mathrm{hpf}$ in heterozygous and mutant embryos.

\subsubsection{Is the npas 4 l reporter expressed in lymphangioblasts?}

npas $4 l$ is crucial for the induction of embryonic endothelial cell specification during early vasculogenesis. However, it has been described that during the formation of the facial lymphatics, lymphangioblasts specify in the surrounding tissue and are recruited to the tip of the lymphatic sprout. It should be enlightening to observe whether the npas $4 \mathrm{l}$ reporter is expressed in these specialized endothelial cells or restricted to early embryonic processes. If npas $4 \mathrm{l}$ is not expressed in these cells, it might be worthwhile to investigate whether these specialized endothelial cells can specify in npas $4 l$ mutants.

\subsubsection{Is there a common progenitor of endothelial and blood cells?}

Whether hematopoietic and endothelial cells are derived from different progenitors (Parker and Stainier, 1999) or share a common progenitor termed hemangioblast (Vogeli et al., 2006) is debated. We have antibodies for the Fli1a and Gatala - two of the earliest markers available. With a simple antibodystaining, I would like to determine whether Fli1a and Gata1a colocalize in the early endothelial progenitors or whether they form distinct populations. This experiment may provide another hint as to whether or not endothelial and hematopoietic cells share a common progenitor population in the early embryo.

\subsubsection{Is the fli1a:GFP transgene transiently present in en- dothelial progenitors of npas 4 l mutants?}

fli1a has been reported to be initially expressed in the posterior lateral plate mesoderm of npas 4 l mutants at the 6 somite stage (Liu et al., 2008). It would be interesting to know whether the transgenic fli1a:GFP reporter line can be detected in npas $4 l$ mutants at the same stage as well. If the reporter 
was expressed, it would give us the ability to screen for animals that show GFP expression in the posterior but not the anterior lateral plate mesoderm, thereby allowing very early genotyping of living npas $4 \mathrm{l}$ mutants by fluorescence. This would provide a new important tool to study early npas $4 l$ mutant embryos and for sorting large numbers of early mutant embryos for certain analyses such as proteomics.

\subsubsection{Does the $n$ pas $4 l$ reporter label all cardiomyocytes in the first heart field?}

The npas $4 l$ reporter is expressed in some but not all cardiomyocytes, especially in those close to the atrioventricular canal at $48 \mathrm{hpf}$. However, while imaging $24 \mathrm{hpf}$ embryos, I noticed that all cardiomyocytes were labeled by the reporter at this stage, visible as two distinct layers of npas $4 l$ reporter positive cells representing the endocardium and myocardium. This may suggest that npas $4 l$ is expressed only in cardiomyocytes of the first heart field. To test this, I intend to cross the npas 4 l reporter to a myl'7 reporter background and analyze whether all cells positive for the cardiomyocyte marker express the npas $4 l$ reporter as well. 



\section{Chapter 6}

\section{Conclusion}

This thesis describes the generation of new tools to investigate the master regulator of the endothelial cell fate npas 4 l. The following aims were successfully achieved:

1. Generate a tagged Npas4l-V5 line for protein detection and proteomic analyses. I successfully generated a genomic knock-in line tagging endogenous npas $4 l$ with a V5 antigen that preserved Npas4l function and detected it reliably during early embryogenesis. I decided to postpone proteomic analyses due to time constraints.

2. Visualize npas4l expression in vivo by generating a fluorescent reporter line. I successfully generated an endogenous knock-in of a Gal4-VP16 driver allele into the endogenous npas 4 l locus. The reporter line showed the expected endothelial expression from early stages onwards.

3. Characterize the expression pattern and protein localization. I used the Gal4 reporter allele to trace npas $4 l$ reporter expressing cells in embryos as well as in adults.

4. What happens to npas 4 l expressing cells in npas 4 l mutants? I determined that in the absence of npas $4 l$ endothelial progenitors do not specify or migrate. Instead, they are contributing to the skeletal muscle and pronephric tubules. Single cell RNAseq of the npas $4 l$ reporter positive lineage confirmed that these two lineage changes account for most of the molecular differences observed between npas $4 l$ heterozygotes and npas $4 l$ mutants.

5. How does the npas $4 l$ mutant phenotype compare to the mutant phenotypes of its direct downstream targets etsrp, tal1 
and $\mathbf{l m o} 2$ ? I determined that npas $4 \mathrm{l}$ reporter expressing cells in tal1 mutants fail to migrate. Some of them contribute to the pronephric tubules. In etsrp mutants the cells migrate but fail to differentiate. Some of them contribute to skeletal muscle. In lmo2 mutants, npas 4 l reporter expressing cells migrate and differentiate, but many express pronephric tubule markers. All in all the three mutants phenocopy distinct aspects of the npas $4 l$ mutant phenotype, shedding light on the detailed mechanism of inducing the endothelial cell fate.

In addition to achieving the aims mentioned above, I gained these additional valuable insights regarding npas4l:

- I found that the npas 4 l reporter is re-expressed during regeneration. It could, therefore, be a marker gene for the regenerating blastema or might even have a physiological effect on the regulation of regenerative processes.

- I generated a new $U A S: m T a g B F P 2$ reporter for more flexible multicolor imaging of Gal4-reporters like the npas $4 l$ reporter line. This allows the combination of Gal4 reporters with both GFPand RFP-expressing transgenic reporter lines at the same time.

- I found the first vertebrate mutants completely devoid of en-

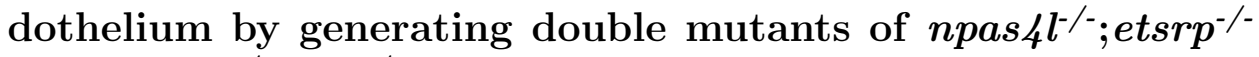
and $\boldsymbol{n p a s} 4 \mathbf{l}^{-/-} ; \boldsymbol{t a l 1}^{-/-}$. Thereby, I determined that the remaining endothelial cells in npas $4 l$ mutants develop through Npas4l independent expression of etsrp in these cells. Also, no endothelial cells can develop in zebrafish in the absence of etsrp and tal1.

- I achieved an almost complete rescue of the vasculogenesis phenotypes in npas 4 l mutants can be achieved by mRNA overexpression of tal1. These initial results suggest that a viable rescue of the npas $4 l$ mutant and, thereby, a study of adult npas $4 l \mathrm{mu}-$ tants may be possible.

- I enahnced endothelial transplantation efficiency by treatment of donors with npas $4 \boldsymbol{l}$-mRNA. Besides being an interesting developmental phenomenon, endothelial and endocardial transplants are technically challenging. The ability to promote the development of endothelial cells by overexpressing npas $4 \mathrm{l}$ mRNA in donor cells could simplify this process significantly. 


\section{Appendix}

\section{List of abbreviations}

Table 6.1: List of abbreviations and most important genes.

\begin{tabular}{l|l} 
Abbreviation & Description \\
\hline AHR & aryl hydrocarbon receptor \\
\hline ALPM & anterior lateral plate mesoderm \\
\hline BFP & blue fluorescent protein \\
\hline bHLH & basic helix-loop-helix (DNA- binding motif) \\
\hline BMP & Chromatin immunoprecipitation \\
\hline ChIP & $\begin{array}{l}\text { describes the npas4l mutant phenotype and was used as } \\
\text { a gene name before } \text { npas4l was discovered }\end{array}$ \\
\hline cloche & dorsal aorta \\
\hline DA & days post fertilization \\
\hline dpf & embryonic day (mouse development) \\
\hline E & endothelial cell \\
\hline EC & electrophoretic mobility shift assay \\
\hline EMSA & $\begin{array}{l}\text { Erythroblast Transformation Specific (DNA-binding mo- } \\
\text { tif and transcription factor family) }\end{array}$ \\
\hline ETS & zebrafish gene encoding the transcription factor Etsrp \\
\hline etsrp & ETS-related protein (zebrafish ETS transcription factor) \\
\hline Etsrp & fluorescence activated cell sorting \\
\hline FACS & false discovery rate \\
\hline FDR & $\begin{array}{l}\text { flanked by Lox recombination sequences from bacterio- } \\
\text { phage P1 }\end{array}$ \\
\hline floxed & F-Box (protein motif and transcription factor class) \\
\hline FOX & green fluorescent protein \\
\hline GFP & hene ontology \\
\hline GO & het
\end{tabular}




\begin{tabular}{|c|c|}
\hline HLH & helix-loop-helix (motif and transcription factor family) \\
\hline hpf & hours post fertilization \\
\hline HRMA & high resolution melt analysis \\
\hline indel & insertion/deletion \\
\hline $\operatorname{lmo2}$ & zebrafish gene encoding the transcription factor Lmo2 \\
\hline Lmo2 & Lim-domain only 2 (zebrafish transcription factor) \\
\hline LPM & lateral plate mesoderm \\
\hline mTagBFP & engineered monomeric blue fluorescent protein \\
\hline mut & mutant \\
\hline n.a. & not analyzed \\
\hline npas4l & zebrafish gene encoding the transcription factor Npas4l \\
\hline Npas4l & $\begin{array}{l}\text { Neuronal PAS-domain protein } 4 \text { like (zebrafish bHLH- } \\
\text { PAS transcription factor) }\end{array}$ \\
\hline PAS & $\begin{array}{l}\text { Per-Arnt-Sim domain (enables protein-protein interac- } \\
\text { tions) }\end{array}$ \\
\hline PCA & principal componen analysis \\
\hline PCR & polymerase chain reaction \\
\hline $\mathrm{PCV}$ & posterior cardinal vein \\
\hline PEST & $\begin{array}{l}\text { prolin, glutamic acid, serine and threonin rich sequence } \\
\text { motif that promotes protein degradation }\end{array}$ \\
\hline PLPM & posterior lateral plate mesoderm \\
\hline qPCR & quantitative realtime $\mathrm{PCR}$ \\
\hline RFP & red fluorescent protein \\
\hline RNAseq & transcriptomic profiling using next generation sequencing \\
\hline scATACseq & $\begin{array}{l}\text { single cell assay for transposase accessible chromatin us- } \\
\text { ing sequencing }\end{array}$ \\
\hline scRNAseq & $\begin{array}{l}\text { single cell transcriptomic profiling using next generation } \\
\text { sequencing }\end{array}$ \\
\hline TAD & transactivation domain \\
\hline tal1 & zebrafish gene encoding the transcription factor Tal1 \\
\hline Tal1 & $\begin{array}{l}\text { T-cell acute lymphocytic leukemia (zebrafish bHLH tran- } \\
\text { scription factor) }\end{array}$ \\
\hline TALEN & transcription activator-like effector nucleases \\
\hline UTR & untranslated region \\
\hline
\end{tabular}




\section{Acknowledgements}

My biggest thank you goes to my girlfriend Saskia for the continuous support over the years despite repeated versions of "I will just need another hour in the lab to finish..." followed by an immediate apology about 4 hours later. You are a great person, very kind, smart and supportive and I am happy to have you in my life.

Although originally, I just came to the Stainier lab to learn how to work with mice (no joke), I decided to do my PhD-thesis in this lab the second Didier offered me the choice to either work on a pre-existing project or to develop my own ideas. Thanks a lot for that opportunity, it is the kind of challenge I looked for for years. In hindsight, the option I took may not have been the smartest one, but even though I struggled for years to find a publishable project, the learning experience was intense and the lab perfect for this kind of search. Special thanks to founder male \#3 who allowed me to finally switch projects and go into the npas $4 l$ field.

While he gave me a lot of space to develop my own ideas, I also appreciate Didier's mentoring, especially since I started writing the paper last year. I find it challenging to stay focused or pay attention to details, but thanks to your feedback, I feel like I am moving in the right direction.

Apart from Didier, I also got great feedback from Bettina, Michelle, Seb and Ruben. Thank you guys for beta reading my thesis and giving me suggestions on how to improve it. And a special thanks to Saskia, again, for reading the entire thesis and correcting hundreds of commas (even in this sentence).

Thanks as well to Ingo Ebersberger, not only for agreeing to review this thesis but also for the collaboration and the many discussions about evolution and Bioinformatics. I really appreciate the perspective and feedback!

My colleagues in Bay 3, you made working in the Stainier lab a lot of fun over the years: Seb, who mentored me during my time as an intern and donated parts of his bench space when I did not have an own bench in the lab yet as well as Ayele, Deepika and Veronica. You guys were great and if you read this, Ayele: We should get some pizza slices again with enough chili to make the weird shop owner complain that we ruin him... Also the more recent inhabitants of Bay 3 make for great company. Thanks to Jialing and 
Shengnan! And we have two open spots and are looking forward to new bay mates!

Although it is futile trying to list all the amazing help I got from so many people over the years, I want to highlight at least a few of them: Thanks Sven for co-authoring the cloche project, it really helped point this project in the right direction. Sarah and Hans-Martin often helped me with injections and transplants and also taught me how to do them myself. I really appreciate the help and one day might even convince Hans-Martin that I am not a spy sent from the GDR. Radhan was always there if there were problems or questions concerning microscopy and we had a great time testing out some new and exciting imaging equipment together. Martin and Moni have always been taking great care of my fish in the system. Marianne, Beate, Carmen and Nana all were incredibly helpful as well. And last but now least I am very thankful to Simon and Sabine for helping me to comply with all kinds of regulations we have to deal with daily.

Every nerd needs some people to drink coffee with and go crazy about all kinds of things. Thanks for all the passionate discussions about biology and everything else to Srinath, Sarah, Sven, Michele, Seb, Giulia, Srini, Mohamed, Hadil and Thomas. You guys are great!

Zuletzt möchte ich noch meinen Eltern und Großeltern danken. Ihr habt mich immer unterstützt und mir sogar meine ersten Laborerfahrungen in Berlin ermöglicht. Vielen Dank dafür, dass ihr immer für mich da wart.

That is it, so everything that is left to say is:

So long, and thanks for all the fish!

(Douglas Adams, The fourth book in the Hitch-Hiker trilogy, 1984) 


\section{III curriculum vitae}

\section{General information}

Name: $\quad$ Kenny Mattonet

Date of birth: $\quad 22.02 .1993$

Place of birth: Berlin

Nationality: German

E-Mail: $\quad$ kenny.mattonet@mpi-bn.mpg.de

\section{Education}

2003-2011 "Otto-Nagel-Gymnasium" in Berlin (German Abitur, overall grade 1.4)

1999-2003 "Grundschule am Bürgerpark" in Berlin

\section{Academic training}

2017-now PhD-studies, Dept. III, Max-Planck-Institut for Heart and Lung Research

2014-2016 Studies of Cell Biology, Goethe University Frankfurt am Main, Germany, Master of Science (M.Sc.)

2011-2014 Studies of Biochemistry, University of Hamburg, Germany, Bachelor of Science (B.Sc.)

\section{Research experience}

2015-2016

2014

2012-2013 research assistant (6 months), Acker-Palmer lab, University of Frankfurt visiting researcher (6 months), Alitalo lab, Helsinki research assistant (6 months), Wienand lab, University of Hamburg 


\section{Awards}

2019

2014-2016

2014

2014
GRS Angiogenesis Poster Award "Deutschlandstipendium" (awarded twice)

research grant (3 months) provided by the Alitalo lab research grant (3 months) by the Finnish Norwegian Medical Foundation

\section{Publications}

Mattonet, K., and Jeltsch, M. (2015). Heterogeneity of the origin of the lymphatic system. LymphForsch19, 84-88.

Jha, S.K., Rauniyar, K., Chronowska, E., Mattonet, K., Maina, E.W., Koistinen, H., Stenman, U.-H., Alitalo, K., and Jeltsch, M. (2019). KLK3/PSA and cathepsin D activate VEGF-C and VEGF-D. Elife8, 1-30.

Batchu, K., Hokynar, K., Jeltsch, M., Mattonet, K., and Somerharju, P. (2015). Substrate efflux propensity is the key determinant of iPLA- $\beta$ mediated glycerophospholipid hydrolysis. The Journal of Biological Chemistry.

Burg, L., Palmer, N., Kikhi, K., Miroshnik, E.S., Rueckert, H., Gaddy, E., MacPherson Cunningham, C., Mattonet, K., Lai, S.-L., Marín-Juez, R., et al. (2018). Conditional mutagenesis by oligonucleotide-mediated integration of loxP sites in zebrafish. PLoS Genet. 14, e1007754. 


\section{Bibliography}

M. J. Abedin, A. Nguyen, N. Jiang, C. E. Perry, J. M. Shelton, D. K. Watson, and A. Ferdous. Fli1 acts downstream of etv2 to govern cell survival and vascular homeostasis via positive autoregulation. Circulation Research, 114(11):1690-1699, May 2014. ISSN 0009-7330. doi: 10.1161/CIRCRESAHA.1134303145. URL https://www.ahajournals . org/doi/10.1161/CIRCRESAHA.1134303145.

R. Agarwala, T. Barrett, J. Beck, D. A. Benson, C. Bollin, E. Bolton, D. Bourexis, J. R. Brister, S. H. Bryant, K. Canese, M. Cavanaugh, C. Charowhas, K. Clark, I. Dondoshansky, M. Feolo, L. Fitzpatrick, K. Funk, L. Y. Geer, V. Gorelenkov, A. Graeff, W. Hlavina, B. Holmes, M. Johnson, B. Kattman, V. Khotomlianski, A. Kimchi, M. Kimelman, M. Kimura, P. Kitts, W. Klimke, A. Kotliarov, S. Krasnov, A. Kuznetsov, M. J. Landrum, D. Landsman, S. Lathrop, J. M. Lee, C. Leubsdorf, Z. Lu, T. L. Madden, A. Marchler-Bauer, A. Malheiro, P. Meric, I. KarschMizrachi, A. Mnev, T. Murphy, R. Orris, J. Ostell, C. O'Sullivan, V. Palanigobu, A. R. Panchenko, L. Phan, B. Pierov, K. D. Pruitt, K. Rodarmer, E. W. Sayers, V. Schneider, C. L. Schoch, G. D. Schuler, S. T. Sherry, K. Siyan, A. Soboleva, V. Soussov, G. Starchenko, T. A. Tatusova, F. Thibaud-Nissen, K. Todorov, B. W. Trawick, D. Vakatov, M. Ward, E. Yaschenko, A. Zasypkin, and K. Zbicz. Database resources of the National Center for Biotechnology Information. Nucleic Acids Research, 46 (D1):D8-D13, jan 2018. ISSN 0305-1048. doi: 10.1093/nar/gkx1095. URL https ://academic .oup.com/nar/article/46/D1/D8/4621330.

S. F. Altschul, W. Gish, W. Miller, E. W. Myers, and D. J. Lipman. Basic local alignment search tool. Journal of Molecular Biology, 215 (3):403-410, oct 1990. ISSN 00222836. doi: 10.1016/S0022-2836(05) 80360-2. URL https://linkinghub.elsevier.com/retrieve/pii/ S0022283605803602.

K. Ando, S. Fukuhara, N. Izumi, H. Nakajima, H. Fukui, R. N. Kelsh, and N. Mochizuki. Clarification of mural cell coverage of vascular en- 
dothelial cells by live imaging of zebrafish. Development, pages 13281339, 2016. ISSN 0950-1991. doi: 10.1242/dev.132654. URL http: //dev.biologists.org/cgi/doi/10.1242/dev.132654.

K. Asakawa, M. L. Suster, K. Mizusawa, S. Nagayoshi, T. Kotani, A. Urasaki, Y. Kishimoto, M. Hibi, and K. Kawakami. Genetic dissection of neural circuits by Tol2 transposon-mediated Gal4 gene and enhancer trapping in zebrafish. Proceedings of the National Academy of Sciences of the United States of America, 105(4):1255-1260, 2008. ISSN 10916490. doi: 10.1073/ pnas.0704963105.

D. Balciunas, K. J. Wangensteen, A. Wilber, J. Bell, A. Geurts, S. Sivasubbu, X. Wang, P. B. Hackett, D. A. Largaespada, R. S. McIvor, and S. C. Ekker. Harnessing a high cargo-capacity transposon for genetic applications in vertebrates. PLoS genetics, 2(11): e169, nov 2006. ISSN 1553-7404. doi: 10.1371/journal.pgen.0020169. URL http://www.ncbi.nlm.nih.gov/pubmed/17096595http://www . pubmedcentral.nih.gov/articlerender.fcgi?artid=PMC1635535.

K. Baltrunaite, M. P. Craig, S. Palencia Desai, P. Chaturvedi, R. N. Pandey, R. S. Hegde, and S. Sumanas. ETS transcription factors Etv2 and Fli1b are required for tumor angiogenesis. Angiogenesis, 20(3):307-323, 2017. ISSN 15737209. doi: 10.1007/s10456-017-9539-8.

V. L. Bautch. Stem cells and the vasculature. Nature Medicine, 17(11): 1437-1443, 2011. ISSN 10788956. doi: 10.1038/nm.2539.

V. L. Bautch and K. M. Caron. Blood and lymphatic vessel formation. Cold Spring Harbor Perspectives in Biology, 7(3):1-14, 2015. ISSN 19430264. doi: 10.1101/cshperspect.a008268.

V. Bergen, M. Lange, S. Peidli, F. A. Wolf, and F. J. Theis. Generalizing RNA velocity to transient cell states through dynamical modeling. Nature Biotechnology, pages 1-26, 2020. ISSN 15461696. doi: 10.1038/s41587-020-0591-3.

D. C. Bersten, A. E. Sullivan, D. J. Peet, and M. L. Whitelaw. bHLH-PAS proteins in cancer. Nature Reviews Cancer, 13(12):827-841, dec 2013. ISSN 1474-175X. doi: 10.1038/nrc3621. URL http://www . nature.com/ articles/nrc3621.

M. Blum, H.-Y. Chang, S. Chuguransky, T. Grego, S. Kandasaamy, A. Mitchell, G. Nuka, T. Paysan-Lafosse, M. Qureshi, S. Raj, L. Richardson, G. A. Salazar, L. Williams, P. Bork, A. Bridge, J. Gough, D. H. 
Haft, I. Letunic, A. Marchler-Bauer, H. Mi, D. A. Natale, M. Necci, C. A. Orengo, A. P. Pandurangan, C. Rivoire, C. J. A. Sigrist, I. Sillitoe, N. Thanki, P. D. Thomas, S. C. E. Tosatto, C. H. Wu, A. Bateman, and R. D. Finn. The InterPro protein families and domains database: 20 years on. Nucleic Acids Research, 49(D1):D344-D354, 11 2020. ISSN 0305-1048. doi: 10.1093/nar/gkaa977. URL https://doi.org/10.1093/ nar/gkaa977.

L. A. Brown, A. R. Rodaway, T. F. Schilling, T. Jowett, P. W. Ingham, R. K. Patient, and A. D. Sharrocks. Insights into early vasculogenesis revealed by expression of the ETS-domain transcription factor Fli-1 in wild-type and mutant zebrafish embryos. Mechanisms of Development, 90(2):237-252, 2000. ISSN 09254773. doi: 10.1016/S0925-4773(99)00256-7.

L. Burg, K. Zhang, T. Bonawitz, V. Grajevskaja, G. Bellipanni, R. Waring, and D. Balciunas. Internal epitope tagging informed by relative lack of sequence conservation. Scientific Reports, 6:36986, 2016. ISSN 20452322. doi: 10.1038/srep36986. URL http://www.nature.com/articles/ srep36986.

J. Bussmann, J. Bakkers, and S. Schulte-Merker. Early endocardial morphogenesis requires Scl/Tal1. PLoS Genetics, 3(8):1425-1437, 2007. ISSN 15537390. doi: 10.1371/journal.pgen.0030140.

S. J. Capon and K. A. Smith. Endocardial differentiation in zebrafish occurs during early somitogenesis and is dependent on BMP and etv2 signalling. bioRxiv, 2019. doi: 10.1101/654525. URL https://www.biorxiv.org/ content/early/2019/05/30/654525.

S. Casie Chetty and S. Sumanas. Ets1 functions partially redundantly with Etv2 to promote embryonic vasculogenesis and angiogenesis in zebrafish. Developmental Biology, 465(1):11-22, sep 2020. ISSN 00121606. doi: 10.1016/j.ydbio.2020.06.007. URL https://linkinghub.elsevier.com/ retrieve/pii/S0012160620301822.

H. Chagraoui, M. S. Kristiansen, J. P. Ruiz, A. Serra-Barros, J. Richter, E. Hall-Ponselé, N. Gray, D. Waithe, K. Clark, P. Hublitz, E. Repapi, G. Otto, P. Sopp, S. Taylor, S. Thongjuea, P. Vyas, and C. Porcher. SCL/TAL1 cooperates with Polycomb RYBP-PRC1 to suppress alternative lineages in blood-fated cells. Nature Communications, 9(1):117, 2018. ISSN 20411723. doi: 10.1038/s41467-018-07787-6. URL http://dx.doi.org/10.1038/s41467-018-07787-6. 
B. Chestnut and S. Sumanas. Zebrafish etv2 knock-in line labels vascular endothelial and blood progenitor cells. Developmental Dynamics, 249(2): 245-261, feb 2020. ISSN 1058-8388. doi: 10.1002/dvdy.130. URL https: //onlinelibrary.wiley.com/doi/abs/10.1002/dvdy.130.

B. Chestnut, S. Casie Chetty, A. L. Koenig, and S. Sumanas. Single-cell transcriptomic analysis identifies the conversion of zebrafish Etv2-deficient vascular progenitors into skeletal muscle. Nature Communications, 11(1): 2796, 2020. ISSN 2041-1723. doi: 10.1038/s41467-020-16515-y. URL https://doi.org/10.1038/s41467-020-16515-y.

T. U. Consortium. UniProt: the universal protein knowledgebase in 2021. Nucleic Acids Research, 49(D1):D480-D489, 11 2020. ISSN 0305-1048. doi: 10.1093/nar/gkaa1100. URL https://doi.org/10.1093/nar/gkaa1100.

G. Couly, P. Coltey, A. Eichmann, and N. M. Le Douarin. The angiogenic potentials of the cephalic mesoderm and the origin of brain and head blood vessels. Mechanisms of Development, 53(1):97-112, 1995. ISSN 09254773. doi: 10.1016/0925-4773(95)00428-9.

M. P. Craig, V. Grajevskaja, H. K. Liao, J. Balciuniene, S. C. Ekker, J. S. Park, J. J. Essner, D. Balciunas, and S. Sumanas. Etv2 and Fli1b function together as key regulators of vasculogenesis and angiogenesis. Arteriosclerosis, Thrombosis, and Vascular Biology, 35(4):865-876, 2015. ISSN 15244636. doi: 10.1161/ATVBAHA.114.304768.

J. M. Davison, C. M. Akitake, M. G. Goll, J. M. Rhee, N. Gosse, H. Baier, M. E. Halpern, S. D. Leach, and M. J. Parsons. Transactivation from Gal4VP16 transgenic insertions for tissue-specific cell labeling and ablation in zebrafish. Developmental Biology, 304(2):811-824, 2007. ISSN 00121606. doi: 10.1016/j.ydbio.2007.01.033.

S. De Val, N. C. Chi, S. M. Meadows, S. Minovitsky, J. P. Anderson, I. S. Harris, M. L. Ehlers, P. Agarwal, A. Visel, S.-M. Xu, L. A. Pennacchio, I. Dubchak, P. A. Krieg, D. Y. Stainier, and B. L. Black. Combinatorial Regulation of Endothelial Gene Expression by Ets and Forkhead Transcription Factors. Cell, 135(6):1053-1064, dec 2008. ISSN 00928674. doi: 10.1016/j.cell.2008.10.049. URL https://linkinghub.elsevier. com/retrieve/pii/S0092867408013871.

A. Dobin, C. A. Davis, F. Schlesinger, J. Drenkow, C. Zaleski, S. Jha, P. Batut, M. Chaisson, and T. R. Gingeras. STAR: ultrafast universal 
RNA-seq aligner. Bioinformatics, 29(1):15-21, jan 2013. ISSN 13674803. doi: 10.1093/bioinformatics/bts635. URL https://doi.org/10. 1093/bioinformatics/bts635.

T. Dobrzycki, C. B. Mahony, M. Krecsmarik, C. Koyunlar, R. Rispoli, J. Peulen-Zink, K. Gussinklo, B. Fedlaoui, E. de Pater, R. Patient, and R. Monteiro. Deletion of a conserved Gata2 enhancer impairs haemogenic endothelium programming and adult Zebrafish haematopoiesis. Communications Biology, 3(1):71, dec 2020. ISSN 2399-3642. doi: 10.1038/s42003-020-0798-3. URL http://www.nature.com/articles/ s42003-020-0798-3.

K. A. Dooley, A. J. Davidson, and L. I. Zon. Zebrafish scl functions independently in hematopoietic and endothelial development. Developmental Biology, 277(2):522-536, 2005. ISSN 00121606. doi: 10.1016/j.ydbio.2004. 09.004 .

K. M. Draheim, N. Hermance, Y. Yang, E. Arous, J. Calvo, and M. A. Kelliher. A DNA-binding mutant of TAL1 cooperates with LMO2 to cause T cell leukemia in mice. Oncogene, 30(10):1252-1260, mar 2011. ISSN 0950-9232. doi: 10.1038/onc.2010.495. URL https://www.ncbi . nlm.nih.gov/pmc/articles/PMC3624763/pdf/nihms412728.pdfhttps: //linkinghub.elsevier.com/retrieve/pii/S0022202X15370834http: //www.nature.com/articles/onc2010495.

D. J. Dumont, G. J. Gradwohl, G. H. Fong, R. Auerbach, and M. L. Breitman. The endothelial-specific receptor tyrosine kinase, tek, is a member of a new subfamily of receptors. Oncogene, 8(5):1293-301, may 1993. ISSN 0950-9232. URL http://www.ncbi.nlm.nih.gov/pubmed/8386827.

D. J. Dumont, G. Gradwohl, G. H. Fong, M. C. Puri, M. Gertsenstein, A. Auerbach, and M. L. Breitman. Dominant-negative and targeted null mutations in the endothelial receptor tyrosine kinase, tek, reveal a critical role in vasculogenesis of the embryo. Genes \& Development, 8(16):18971909, aug 1994. ISSN 0890-9369. doi: 10.1101/gad.8.16.1897. URL http: //www.genesdev.org/cgi/doi/10.1101/gad.8.16.1897.

S. Dörfler. xmedia recode, 2020. URL https://www.xmedia-recode.de/ en/.

M. A. El-Brolosy, Z. Kontarakis, A. Rossi, C. Kuenne, S. Günther, N. Fukuda, K. Kikhi, G. L. M. Boezio, C. M. Takacs, S.-L. Lai, R. Fukuda, 
C. Gerri, A. J. Giraldez, and D. Y. R. Stainier. Genetic compensation triggered by mutant mRNA degradation. Nature, 568(7751):193197, apr 2019. ISSN 0028-0836. doi: 10.1038/s41586-019-1064-z. URL http://www . nature.com/articles/s41586-019-1064-z.

K. El Omari, S. J. Hoosdally, K. Tuladhar, D. Karia, P. Vyas, R. Patient, C. Porcher, and E. J. Mancini. Structure of the leukemia oncogene LMO2: Implications for the assembly of a hematopoietic transcription factor complex. Blood, 117(7):2146-2156, 2011. ISSN 00064971. doi: 10.1182/blood-2010-07-293357.

E. Ellertsdóttir, A. Lenard, Y. Blum, A. Krudewig, L. Herwig, M. Affolter, and H. G. Belting. Vascular morphogenesis in the zebrafish embryo. Developmental Biology, 341(1):56-65, 2010. ISSN 1095564X. doi: 10.1016/j.ydbio.2009.10.035.

J. Eriksson and J. Löfberg. Development of the hypochord and dorsal aorta in the zebrafish embryo (Danio rerio). Journal of Morphology, 244(3):167176, 2000. ISSN 03622525. doi: 10.1002/(SICI)1097-4687(200006)244: 3〈167::AID-JMOR2 $>3.0 . C O ; 2-J$.

P. Faloon, E. Arentson, A. Kazarov, C. X. Deng, C. Porcher, S. Orkin, and K. Choi. Basic fibroblast growth factor positively regulates hematopoietic development. Development, 127(9):1931-1941, 2000. ISSN 09501991.

N. Ferrara, K. Carver-Moore, H. Chen, M. Dowd, L. Lu, K. S. O'Shea, L. Powell-Braxton, K. J. Hillan, and M. W. Moore. Heterozygous embryonic lethality induced by targeted inactivation of the VEGF gene. Nature, 380(6573):439-442, apr 1996. ISSN 0028-0836. doi: 10.1038/380439a0. URL http: //www . nature.com/articles/380439a0.

N. Ferrara, H. P. Gerber, and J. LeCouter. The biology of VEGF and its receptors. Nat Med, 9(6):669-676, 2003. ISSN 1078-8956. doi: 10.1038/ nm0603-669. URL http://www.ncbi.nlm.nih.gov/pubmed/12778165.

V. Fortuna, L. Pardanaud, I. Brunet, R. Ola, E. Ristori, M. M. Santoro, S. Nicoli, and A. Eichmann. Vascular Mural Cells Promote Noradrenergic Differentiation of Embryonic Sympathetic Neurons. Cell Reports, 11(11): 1786-1796, 2015. ISSN 22111247. doi: 10.1016/j.celrep.2015.05.028. URL http://dx.doi.org/10.1016/j.celrep.2015.05.028.

J. L. Fribourgh and C. L. Partch. Assembly and function of bHLH-PAS complexes. Proceedings of the National Academy of Sciences, 114(21): 
5330-5332, may 2017. ISSN 0027-8424. doi: 10.1073/pnas.1705408114. URL http://www.pnas.org/lookup/doi/10.1073/pnas.1705408114.

J. A. Gagnon, E. Valen, S. B. Thyme, P. Huang, L. Ahkmetova, A. Pauli, T. G. Montague, S. Zimmerman, C. Richter, and A. F. Schier. Efficient mutagenesis by Cas9 protein-mediated oligonucleotide insertion and largescale assessment of single-guide RNAs. PLoS ONE, 9(5):5-12, 2014. ISSN 19326203. doi: 10.1371/journal.pone.0098186.

M. Gering, A. Rodaway, B. Göttgens, R. Patient, and A. Green. The SCL gene specifies haemangioblast development from early mesoderm. The EMBO Journal, 17(14):4029-4045, jul 1998. ISSN 0261-4189. doi: 10. 1093/emboj/17.14.4029. URL http://emboj.embopress.org/cgi/doi/ $10.1093 / \mathrm{emboj} / 17.14 .4029$.

M. Gering, Y. Yamada, T. H. Rabbitts, and R. K. Patient. Lmo2 and Scl/Tal1 convert non-axial mesoderm into haemangioblasts which differentiate into endothelial cells in the absence of Gata1. Development, 130 (25):6187-6199, 2003. ISSN 09501991. doi: 10.1242/dev.00875.

I. Geudens, B. Coxam, S. Alt, V. Gebala, A.-C. Vion, K. Meier, A. Rosa, and H. Gerhardt. Artery-vein specification in the zebrafish trunk is prepatterned by heterogeneous Notch activity and balanced by flow-mediated fine-tuning. Development, 146(16):dev181024, 2019. ISSN 0950-1991. doi: 10.1242/dev.181024.

F. Gunawan, A. Gentile, R. Fukuda, A. T. Tsedeke, V. Jiménez-Amilburu, R. Ramadass, A. Iida, A. Sehara-Fujisawa, and D. Y. Stainier. Focal adhesions are essential to drive zebrafish heart valve morphogenesis. Journal of Cell Biology, 218(3):1039-1054, mar 2019. ISSN 0021-9525. doi: 10.1083/ jcb.201807175. URL https://rupress.org/jcb/article/218/3/1039/ 120797/Focal-adhesions-are-essential-to-drive-zebrafish.

H. Habeck, J. Odenthal, B. Walderich, H.-M. Maischein, and S. SchulteMerker. Analysis of a Zebrafish VEGF Receptor Mutant Reveals Specific Disruption of Angiogenesis. Current Biology, 12(16):1405-1412, aug 2002. ISSN 09609822. doi: 10.1016/S0960-9822(02)01044-8. URL https:// linkinghub.elsevier.com/retrieve/pii/S0960982202010448.

M. E. Halpern, R. K. Ho, C. Walker, and C. B. Kimmel. Induction of muscle pioneers and floor plate is distinguished by the zebrafish no tail mutation. Cell, 75(1):99-111, oct 1993. ISSN 0092-8674. URL http: //www.ncbi.nlm.nih.gov/pubmed/8402905. 
T. Hasegawa, T. Nakajima, T. Ishida, A. Kudo, and A. Kawakami. A diffusible signal derived from hematopoietic cells supports the survival and proliferation of regenerative cells during zebrafish fin fold regeneration. Developmental Biology, 399(1):80-90, 2015. ISSN 1095564X. doi: 10.1016/j.ydbio.2014.12.015. URL http://dx.doi.org/10.1016/j . ydbio.2014.12.015.

C. S. Helker, A. Schuermann, T. Karpanen, D. Zeuschner, H. G. Belting, M. Affolter, S. Schulte-Merker, and W. Herzog. The zebrafish common cardinal veins develop by a novel mechanism: Lumen ensheathment. Development (Cambridge), 140(13):2776-2786, 2013. ISSN 09501991. doi: 10.1242/dev.091876.

C. S. Helker, A. Schuermann, C. Pollmann, S. C. Chng, F. Kiefer, B. Reversade, and W. Herzog. The hormonal peptide Elabela guides angioblasts to the midline during vasculogenesis. eLife, 4:1-13, 2015. doi: 10.7554/elife.06726.

J. A. Henry Oswald. Sharelatex, 2015. URL https://github.com/ overleaf/overleaf.

S. P. Herbert and D. Y. R. Stainier. Molecular control of endothelial cell behaviour during blood vessel morphogenesis. Nature Reviews. Molecular Cell Biology, 12(9):551-564, sep 2011. ISSN 1471-0080. doi: 10.1038/ nrm3176. URL http://www.ncbi.nlm.nih.gov/pubmed/21860391.

B. M. Hogan and S. Schulte-Merker. How to Plumb a Pisces: Understanding Vascular Development and Disease Using Zebrafish Embryos. Developmental Cell, 42(6):567-583, 2017. ISSN 18781551. doi: 10.1016/j.devcel.2017. 08.015. URL http://dx.doi.org/10.1016/j.devcel.2017.08.015.

E. J. Horstick, D. C. Jordan, S. A. Bergeron, K. M. Tabor, M. Serpe, B. Feldman, and H. A. Burgess. Increased functional protein expression using nucleotide sequence features enriched in highly expressed genes in zebrafish. Nucleic Acids Research, 43(7):e48-e48, 01 2015. ISSN 0305-1048. doi: 10.1093/nar/gkv035. URL https://doi .org/10.1093/nar/gkv035.

W. Y. Hwang, Y. Fu, D. Reyon, M. L. Maeder, S. Q. Tsai, J. D. Sander, R. T. Peterson, J.-R. J. Yeh, and J. K. Joung. Efficient genome editing in zebrafish using a CRISPR-Cas system. Nature Biotechnology, 31(3): 227-229, mar 2013. ISSN 1087-0156. doi: 10.1038/nbt.2501. URL http: //www.nature.com/articles/nbt. 2501. 
A. Inc. Anaconda software distribution, 2020. URL https://docs. anaconda.com/.

S. Isogai, M. Horiguchi, and B. M. Weinstein. The vascular anatomy of the developing zebrafish: an atlas of embryonic and early larval development. Developmental biology, 230(2):278-301, 2001. ISSN 00121606. doi: 10. 1006/dbio.2000.9995.

M. Jacomy, T. Venturini, S. Heymann, and M. Bastian. ForceAtlas2, a Continuous Graph Layout Algorithm for Handy Network Visualization Designed for the Gephi Software. PLOS ONE, 9(6):1-12, 2014. doi: 10. 1371/journal.pone.0098679. URL https://doi.org/10.1371/journal. pone. 0098679 .

L.-E. Jao, S. R. Wente, and W. Chen. Efficient multiplex biallelic zebrafish genome editing using a CRISPR nuclease system. Proceedings of the National Academy of Sciences, 110(34):1390413909, aug 2013. ISSN 0027-8424. doi: 10.1073/pnas.1308335110. URL http://www.ncbi.nlm.nih.gov/pubmed/3752207http: //www .pnas.org/lookup/doi/10.1073/pnas. 1308335110.

L. D. Jensen, Z. Cao, M. Nakamura, Y. Yang, L. Bräutigam, P. Andersson, Y. Zhang, E. Wahlberg, T. Länne, K. Hosaka, and Y. Cao. Opposing Effects of Circadian Clock Genes Bmal1 and Period2 in Regulation of VEGF-Dependent Angiogenesis in Developing Zebrafish. Cell Reports, 2(2):231-241, aug 2012. ISSN 22111247. doi: 10.1016/j.celrep. 2012.07.005. URL https://linkinghub.elsevier.com/retrieve/pii/ S221112471200201X.

Z. Jiang, T. Evans, A. M. Savage, M. Loose, T. J. Chico, F. J. van Eeden, and R. N. Wilkinson. foxc1a and foxc1b differentially regulate angiogenesis from arteries and veins by modulating vascular endothelial growth factor signalling. bioRxiv, 2018. doi: 10.1101/417931. URL https://www. biorxiv.org/content/early/2018/09/14/417931.

Z. Jiang, C. Carlantoni, S. Allanki, I. Ebersberger, and D. Y. R. Stainier. Tek (Tie2) is not required for cardiovascular development in zebrafish. Development, 147(19):dev193029, oct 2020. ISSN 0950-1991. doi: 10.1242/ dev.193029. URL http://dev.biologists.org/lookup/doi/10.1242/ dev. 193029.

S. W. Jin and C. Patterson. The opening act: Vasculogenesis and the origins of circulation. Arteriosclerosis, Thrombosis, and Vascular Biology, 29(5): 623-629, 2009. ISSN 10795642. doi: 10.1161/ATVBAHA.107.161539. 
S.-W. Jin, D. Beis, T. Mitchell, J.-N. Chen, and D. Y. R. Stainier. Cellular and molecular analyses of vascular tube and lumen formation in zebrafish. Development (Cambridge, England), 132(23):5199-5209, dec 2005. ISSN 0950-1991. doi: 10.1242/dev.02087. URL http://www.ncbi.nlm.nih. gov/pubmed/16251212.

J. B. Johnston and S. W. Johnson. The Course of the Blood Flow in Lumbricus. The American Naturalist, 36(424):317-328, apr 1902. ISSN 0003-0147. doi: 10.1086/278121. URL https://www.journals.uchicago.edu/doi/ $10.1086 / 278121$.

M. A. Juarez, F. Su, S. Chun, M. J. Kiel, and S. E. Lyons. Distinct roles for SCL in erythroid specification and maturation in zebrafish. Journal of Biological Chemistry, 280(50):41636-41644, 2005. ISSN 00219258. doi: 10.1074/jbc.M507998200.

A. Khan, O. Fornes, A. Stigliani, M. Gheorghe, J. A. Castro-Mondragon, R. van der Lee, A. Bessy, J. Chèneby, S. R. Kulkarni, G. Tan, D. Baranasic, D. J. Arenillas, A. Sandelin, K. Vandepoele, B. Lenhard, B. Ballester, W. W. Wasserman, F. Parcy, and A. Mathelier. JASPAR 2018: update of the open-access database of transcription factor binding profiles and its web framework. Nucleic acids research, 46 (D1):D260-D266, 2018. ISSN 1362-4962. doi: 10.1093/nar/gkx1126. URL http://www.ncbi.nlm.nih.gov/pubmed/29140473http://www . pubmedcentral.nih.gov/articlerender.fcgi?artid=PMC5753243.

Y. Kikuchi, A. Agathon, J. Alexander, C. Thisse, S. Waldron, D. Yelon, B. Thisse, and D. Y. Stainier. casanova encodes a novel Sox-related protein necessary and sufficient for early endoderm formation in zebrafish. Genes and Development, 15(12):1493-1505, 2001. ISSN 08909369. doi: 10.1101/ $\operatorname{gad} .892301$.

C. B. Kimmel, W. W. Ballard, S. R. Kimmel, B. Ullmann, and T. F. Schilling. Stages of embryonic development of the zebrafish. Developmental Dynamics: An Official Publication of the American Association of Anatomists, 203(3):253-310, jul 1995. ISSN 1058-8388. doi: 10.1002/aja.1002030302. URL http://www.ncbi.nlm.nih.gov/pubmed/8589427.

Y. Kishimoto, K. H. Lee, L. Zon, M. Hammerschmidt, and S. Schulte-Merker. The molecular nature of zebrafish swirl: BMP2 function is essential during early dorsoventral patterning. Development, 124(22):4457-4466, 1997. ISSN 0950-1991. URL https://dev.biologists.org/content/124/22/ 4457. 
F. O. Kok, M. Shin, C.-W. Ni, A. Gupta, A. S. Grosse, A. van Impel, B. C. Kirchmaier, J. Peterson-Maduro, G. Kourkoulis, I. Male, D. F. DeSantis, S. Sheppard-Tindell, L. Ebarasi, C. Betsholtz, S. Schulte-Merker, S. A. Wolfe, and N. D. Lawson. Reverse genetic screening reveals poor correlation between morpholino-induced and mutant phenotypes in zebrafish. Developmental Cell, 32(1):97-108, jan 2015. ISSN 1878-1551. doi: 10.1016/j.devcel.2014.11.018. URL http://www.ncbi.nlm.nih.gov/ pubmed/25533206.

N. Koyano-Nakagawa and D. J. Garry. Etv2 as an essential regulator of mesodermal lineage development. Cardiovascular Research, 113(11):12941306, 2017. ISSN 17553245. doi: 10.1093/cvr/cvx133.

S. W. Kuan, K. Proulx, M. S. Rost, and S. Sumanas. Identification of vasculature-specific genes by microarray analysis of Etsrp/Etv2 overexpressing zebrafish embryos. Developmental Dynamics, 238(7):1836-1850, 2009. ISSN 10588388. doi: 10.1002/dvdy.21990.

A. M. Küchler, E. Gjini, J. Peterson-Maduro, B. Cancilla, H. Wolburg, and S. Schulte-Merker. Development of the Zebrafish Lymphatic System Requires Vegfc Signaling. Current Biology, 16(12):12441248, jun 2006. ISSN 0960-9822. doi: 10.1016/j.cub.2006.05.026. URL http://www.sciencedirect.com/science/article/pii/ S0960982206016095http://www.sciencedirect.com/science/ article/pii/S0960982206016095\{\\#\}.

G. La Manno, R. Soldatov, A. Zeisel, E. Braun, H. Hochgerner, V. Petukhov, K. Lidschreiber, M. E. Kastriti, P. Lönnerberg, A. Furlan, J. Fan, L. E. Borm, Z. Liu, D. van Bruggen, J. Guo, X. He, R. Barker, E. Sundström, G. Castelo-Branco, P. Cramer, I. Adameyko, S. Linnarsson, and P. V. Kharchenko. RNA velocity of single cells. Nature, 560(7719):494-498, 2018. ISSN 14764687. doi: 10.1038/s41586-018-0414-6. URL http://dx . doi.org/10.1038/s41586-018-0414-6.

K. Labun, T. G. Montague, J. A. Gagnon, S. B. Thyme, and E. Valen. CHOPCHOP v2: a web tool for the next generation of CRISPR genome engineering. Nucleic acids research, 44(W1):W272-W276, 2016. ISSN 13624962. doi: 10.1093/nar/gkw398.

N. D. Lawson and B. M. Weinstein. In vivo imaging of embryonic vascular development using transgenic zebrafish. Developmental Biology, 248(2): 307-318, 2002. ISSN 00121606. doi: 10.1006/dbio.2002.0711. 
A. Lebcir, R. Hassan-Abdi, C. Yanicostas, and N. Soussi-Yanicostas. A Rapid and Efficient Method of Identifying G0 Males with Mosaic Germ Line Cells. Zebrafish, 13(6):535-536, 2016. ISSN 1557-8542. doi: 10.1089/ zeb.2016.1363. URL http://www.ncbi.nlm.nih.gov/pubmed/27673347.

D. Lee, C. Park, H. Lee, J. J. Lugus, S. H. Kim, E. Arentson, Y. S. Chung, G. Gomez, M. Kyba, S. Lin, R. Janknecht, D. S. Lim, and K. Choi. ER71 Acts Downstream of BMP, Notch, and Wnt Signaling in Blood and Vessel Progenitor Specification. Cell Stem Cell, 2(5):497-507, 2008. ISSN 19345909. doi: 10.1016/j.stem.2008.03.008.

I. Letunic, S. Khedkar, and P. Bork. SMART: recent updates, new developments and status in 2020. Nucleic Acids Research, 49(D1):D458D460, jan 2021. ISSN 0305-1048. doi: 10.1093/nar/gkaa937. URL https://academic.oup.com/nar/article/49/D1/D458/5940513.

J. Li, Y. Yue, X. Dong, W. Jia, K. Li, D. Liang, Z. Dong, X. Wang, X. Nan, Q. Zhang, and Q. Zhao. Zebrafish foxc1a Plays a Crucial Role in Early Somitogenesis by Restricting the Expression of aldh1a2 Directly. Journal of Biological Chemistry, 290(16):10216-10228, apr 2015. ISSN 00219258. doi: 10.1074/jbc.M114.612572. URL https://linkinghub.elsevier. com/retrieve/pii/S0021925820426730.

L. Li, D. Chen, J. Li, X. Wang, N. Wang, C. Xu, and Q. K. Wang. Aggf1 acts at the top of the genetic regulatory hierarchy in specification of hemangioblasts in zebrafish. Blood, 123(4):501-508, 2014. ISSN 15280020. doi: 10.1182/blood-2013-07-514612.

E. C. Liao, B. H. Paw, A. C. Oates, S. J. Pratt, J. H. Postlethwait, and L. I. Zon. SCL/Tal-1 transcription factor acts downstream of cloche to specify hematopoietic and vascular progenitors in zebrafish. Genes and Development, 12(5):621-626, 1998. ISSN 08909369. doi: 10.1101/gad.12. 5.621 .

W. Liao, B. W. Bisgrove, H. Sawyer, B. Hug, B. Bell, K. Peters, D. J. Grunwald, and D. Y. Stainier. The zebrafish gene cloche acts upstream of a flk-1 homologue to regulate endothelial cell differentiation. Development, 124(2):381-389, 1997. ISSN 09501991.

W. Liao, C. Y. Ho, Y. L. Yan, J. Postlethwait, and D. Y. Stainier. Hhex and scl function in parallel to regulate early endothelial and blood differentiation in zebrafish. Development, 127(20):4303-4313, 2000. ISSN 09501991. 
F. Liu, M. Walmsley, A. Rodaway, and R. Patient. Fli1 Acts at the Top of the Transcriptional Network Driving Blood and Endothelial Development. Current Biology, 18(16):1234-1240, 2008. ISSN 09609822. doi: 10.1016/j. cub.2008.07.048.

F. Liu, I. Kang, C. Park, L. W. Chang, W. Wang, D. Lee, D. S. Lim, D. Vittet, J. M. Nerbonne, and K. Choi. ER71 specifies Flk-1+ hemangiogenic mesoderm by inhibiting cardiac mesoderm and Wnt signaling. Blood, 119(14):3295-3305, 2012. ISSN 00064971. doi: 10.1182/ blood-2012-01-403766.

F. Liu, D. Li, Y. Y. L. Yu, I. Kang, M. Cha, J. Y. Kim, C. Park, D. K. Watson, T. Wang, and K. Choi. Induction of hematopoietic and endothelial cell program orchestrated by ETS transcription factor ER 71/ ETV 2 . EMBO reports, 16(5):654-669, 2015. ISSN 1469-221X. doi: 10.15252/embr.201439939.

N. Ma, Z. Huang, X. Chen, F. He, K. Wang, W. Liu, L. Zhao, X. Xu, W. Liao, H. Ruan, S. Luo, and W. Zhang. Characterization of a Weak Allele of Zebrafish cloche Mutant. PLoS ONE, 6(11):e27540, nov 2011. ISSN 1932-6203. doi: 10.1371/journal.pone.0027540. URL https://dx. plos.org/10.1371/journal.pone.0027540.

M. Marass, A. Beisaw, C. Gerri, F. Luzzani, N. Fukuda, S. Günther, C. Kuenne, S. Reischauer, and D. Y. R. Stainier. Genome-wide strategies reveal target genes of Npas4l associated with vascular development in zebrafish. Development, page dev.173427, 2019. ISSN 0950-1991. doi: 10.1242/dev.173427. URL http://dev.biologists.org/lookup/ doi/10.1242/dev.173427.

D. Marchi, K. Santhakumar, E. Markham, N. Li, K.-H. Storbeck, N. Krone, V. T. Cunliffe, and F. J. M. van Eeden. Bidirectional crosstalk between Hypoxia-Inducible Factor and glucocorticoid signalling in zebrafish larvae. PLOS Genetics, 16(5):e1008757, may 2020. ISSN 1553-7404. doi: 10.1371/ journal.pgen.1008757. URL https://dx.plos.org/10.1371/journal. pgen. 1008757.

M. E. Massari and C. Murre. Helix-Loop-Helix Proteins: Regulators of Transcription in Eucaryotic Organisms. Molecular and Cellular Biology, 20(2): 429-440, jan 2000. ISSN 1098-5549. doi: 10.1128/MCB.20.2.429-440.2000. URL https://mcb.asm.org/content/20/2/429. 
R. Mateus, R. Lourenço, Y. Fang, G. Brito, A. Farinho, F. Valerio, and A. Jacinto. Control of tissue growth by Yap relies on cell density and F-actin in zebrafish fin regeneration. Development (Cambridge), 142(16): 2752-2763, 2015. ISSN 14779129. doi: 10.1242/dev.119701.

G. Matrone, S. Meng, Q. Gu, J. Lv, L. Fang, K. Chen, and J. P. Cooke. Lmo2 (LIM-domain-only 2) modulates Sphk1 (Sphingosine kinase) and promotes endothelial cell migration. Arteriosclerosis, Thrombosis, and Vascular Biology, 37(10):1860-1868, 2017. ISSN 15244636. doi: 10.1161/ATVBAHA. 117.309609.

R. L. Matsuoka, M. Marass, A. Avdesh, C. S. Helker, H.-M. Maischein, A. S. Grosse, H. Kaur, N. D. Lawson, W. Herzog, and D. Y. Stainier. Radial glia regulate vascular patterning around the developing spinal cord. eLife, 5, nov 2016. ISSN 2050-084X. doi: 10.7554/eLife.20253. URL https://elifesciences.org/articles/20253.

A. C. McGarvey, W. Kopp, D. Vučićević, R. Kempfer, K. Mattonet, A. Hirsekorn, I. Bilić, A. Trinks, A. M. Merks, D. Panáková, A. Pombo, A. Akalin, J. P. Junker, D. Y. R. Stainier, D. Garfield, U. Ohler, and S. A. Lacadie. Single-cell-resolved dynamics of chromatin architecture delineate cell and regulatory states in wildtype and cloche/npas4l mutant zebrafish embryos. bioRxiv, page 2020.06.26.173377, jan 2020. doi: 10.1101/2020.06.26.173377. URL http://biorxiv.org/content/early/ 2020/06/26/2020.06.26.173377. abstract.

L. McInnes, J. Healy, N. Saul, and L. Großberger. UMAP: Uniform Manifold Approximation and Projection. Journal of Open Source Software, 3(29): 861, 2018. doi: 10.21105/joss.00861. URL https://doi.org/10.21105/ joss. 00861.

C. Megill, B. Martin, C. Weaver, S. Bell, S. Badajoz, M. Weiden, J. Kiggins, J. Freeman, Fionagriffin, and Bmccandless. chanzuckerberg/cellxgene: Release 0.15.0, 2019.

R. Monahan-Earley, A. M. Dvorak, and W. C. Aird. Evolutionary origins of the blood vascular system and endothelium. Journal of Thrombosis and Haemostasis, 11(SUPPL.1):46-66, 2013. ISSN 15387933. doi: 10.1111/ jth.12253.

M. A. Moreno-Mateos, C. E. Vejnar, J.-D. Beaudoin, J. P. Fernandez, E. K. Mis, M. K. Khokha, and A. J. Giraldez. CRISPRscan: designing highly efficient sgRNAs for CRISPR-Cas9 targeting in vivo. Nature Methods, 12 
(10):982-988, oct 2015. ISSN 1548-7091. doi: 10.1038/nmeth.3543. URL http://www . nature.com/articles/nmeth. 3543.

S. P. Mudumana, D. Hentschel, Y. Liu, A. Vasilyev, and I. A. Drummond. Odd skipped related1 reveals a novel role for endoderm in regulating kidney versus vascular cell fate. Development, 135(20):3355-3367, 2008. ISSN 09501991. doi: 10.1242/dev.022830.

M. C. Mullins, M. Hammerschmidt, D. A. Kane, J. Odenthal, M. Brand, F. J. van Eeden, M. Furutani-Seiki, M. Granato, P. Haffter, C. P. Heisenberg, Y. J. Jiang, R. N. Kelsh, and C. Nusslein-Volhard. Genes establishing dorsoventral pattern formation in the zebrafish embryo: the ventral specifying genes. Development, 123(1):81-93, 1996. ISSN 0950-1991. URL https://dev. biologists.org/content/123/1/81.

P. D. Nguyen, G. E. Hollway, C. Sonntag, L. B. Miles, T. E. Hall, S. Berger, K. J. Fernandez, D. B. Gurevich, N. J. Cole, S. Alaei, M. Ramialison, R. L. Sutherland, J. M. Polo, G. J. Lieschke, and P. D. Currie. Haematopoietic stem cell induction by somite-derived endothelial cells controlled by meox1. Nature, 512(7514):314-318, 2014. ISSN 14764687. doi: 10.1038/nature13678. URL http://dx.doi.org/10.1038/nature13678.

J. Nicenboim, G. Malkinson, T. Lupo, L. Asaf, Y. Sela, O. Mayseless, L. Gibbs-Bar, N. Senderovich, T. Hashimshony, M. Shin, A. Jerafi-Vider, I. Avraham-Davidi, V. Krupalnik, R. Hofi, G. Almog, J. W. Astin, O. Golani, S. Ben-Dor, P. S. Crosier, W. Herzog, N. D. Lawson, J. H. Hanna, I. Yanai, and K. Yaniv. Lymphatic vessels arise from specialized angioblasts within a venous niche. Nature, 522(7554):56-61, 2015. ISSN 14764687. doi: 10.1038/nature14425.

K. S. Okuda, J. W. Astin, J. P. Misa, M. V. Flores, K. E. Crosier, and P. S. Crosier. lyve1 expression reveals novel lymphatic vessels and new mechanisms for lymphatic vessel development in zebrafish. Development (Cambridge, England), 139(13):2381-2391, jul 2012. ISSN 1477-9129. doi: 10.1242/dev.077701. URL http://www.ncbi.nlm.nih.gov/pubmed/ 22627281.

N. A. O'Leary, M. W. Wright, J. R. Brister, S. Ciufo, D. Haddad, R. McVeigh, B. Rajput, B. Robbertse, B. Smith-White, D. Ako-Adjei, A. Astashyn, A. Badretdin, Y. Bao, O. Blinkova, V. Brover, V. Chetvernin, J. Choi, E. Cox, O. Ermolaeva, C. M. Farrell, T. Goldfarb, T. Gupta, D. Haft, E. Hatcher, W. Hlavina, V. S. Joardar, V. K. Kodali, W. Li, D. Maglott, P. Masterson, K. M. McGarvey, M. R. Murphy, K. O’Neill, 
S. Pujar, S. H. Rangwala, D. Rausch, L. D. Riddick, C. Schoch, A. Shkeda, S. S. Storz, H. Sun, F. Thibaud-Nissen, I. Tolstoy, R. E. Tully, A. R. Vatsan, C. Wallin, D. Webb, W. Wu, M. J. Landrum, A. Kimchi, T. Tatusova, M. DiCuccio, P. Kitts, T. D. Murphy, and K. D. Pruitt. Reference sequence (RefSeq) database at NCBI: current status, taxonomic expansion, and functional annotation. Nucleic Acids Research, 44(D1):D733D745, 11 2015. ISSN 0305-1048. doi: 10.1093/nar/gkv1189. URL https://doi.org/10.1093/nar/gkv1189.

H. Osada, G. Grutz, H. Axelson, A. Forster, and T. H. Rabbitts. Association of erythroid transcription factors: Complexes involving the LIM protein RBTN2 and the zinc-finger protein GATA1. Proceedings of the National Academy of Sciences of the United States of America, 92(21):9585-9589, 1995. ISSN 00278424. doi: 10.1073/pnas.92.21.9585.

B. Pak, C. E. Schmitt, W. Choi, J.-D. Kim, O. Han, J. Alsiö, D.-W. Jung, D. R. Williams, W. Coppieters, D. Y. R. Stainier, and S.-W. Jin. Analyses of Avascular Mutants Reveal Unique Transcriptomic Signature of Nonconventional Endothelial Cells, 2020. URL https://www.frontiersin. org/article/10.3389/fcell.2020.589717.

S. Palencia-Desai, V. Kohli, J. Kang, N. C. Chi, B. L. Black, and S. Sumanas. Vascular endothelial and endocardial progenitors differentiate as cardiomyocytes in the absence of Etsrp/Etv2 function. Development, 138(21): 4721-4732, 2011. ISSN 0950-1991. doi: 10.1242/dev.064998. URL http://dev.biologists.org/cgi/doi/10.1242/dev. 064998.

L. Parker and D. Y. Stainier. Cell-autonomous and non-autonomous requirements for the zebrafish gene cloche in hematopoiesis. Development (Cambridge, England), 126(12):2643-51, 1999. ISSN 0950-1991. URL http://www.ncbi.nlm.nih.gov/pubmed/10331976.

L. J. Patterson, M. Gering, C. E. Eckfeldt, A. R. Green, C. M. Verfaillie, S. C. Ekker, and R. Patient. The transcription factors Scl and Lmo2 act together during development of the hemangioblast in zebrafish. Blood, 109 (6):2389-2398, 2007. ISSN 00064971. doi: 10.1182/blood-2006-02-003087.

E. A. Perens, Z. V. Garavito-Aguilar, G. P. Guio-Vega, K. T. Peña, Y. L. Schindler, and D. Yelon. Hand2 inhibits kidney specification while promoting vein formation within the posterior mesoderm. eLife, 5(NOVEMBER2016):1-21, 2016. ISSN 2050084X. doi: 10.7554/eLife. 19941. 
V. N. Pham, N. D. Lawson, J. W. Mugford, L. Dye, D. Castranova, B. Lo, and B. M. Weinstein. Combinatorial function of ETS transcription factors in the developing vasculature. Developmental Biology, 303(2):772-783, 2007. ISSN 00121606. doi: 10.1016/j.ydbio.2006.10.030.

L. K. Phng and H. Gerhardt. Angiogenesis: A Team Effort Coordinated by Notch. Developmental Cell, 16(2):196-208, 2009. ISSN 15345807. doi: 10.1016/j.devcel.2009.01.015. URL http://dx.doi.org/10.1016/ j.devcel.2009.01.015.

K. Pociute, J. A. Schumacher, and S. Sumanas. Clec14a genetically interacts with Etv2 and Vegf signaling during vasculogenesis and angiogenesis in zebrafish. BMC Developmental Biology, 19(1):6, dec 2019. ISSN 1471-213X. doi: 10.1186/s12861-019-0188-6. URL https://bmcdevbiol. biomedcentral.com/articles/10.1186/s12861-019-0188-6.

M. Posner, M. S. McDonald, K. L. Murray, and A. J. Kiss. Why does the zebrafish cloche mutant develop lens cataract? PLoS ONE, 14(3):1-16, 2019. ISSN 19326203. doi: 10.1371/journal.pone.0211399.

I. Project. Inkscape, 2020. URL https://inkscape.org.

K. Proulx, A. Lu, and S. Sumanas. Cranial vasculature in zebrafish forms by angioblast cluster-derived angiogenesis. Developmental Biology, 348(1): 34-46, 2010. ISSN 1095564X. doi: 10.1016/j.ydbio.2010.08.036. URL http://dx.doi.org/10.1016/j.ydbio.2010.08.036.

K. D. Prummel, C. Hess, S. Nieuwenhuize, H. J. Parker, K. W. Rogers, I. Kozmikova, C. Racioppi, E. C. Brombacher, A. Czarkwiani, D. Knapp, S. Burger, E. Chiavacci, G. Shah, A. Burger, J. Huisken, M. H. Yun, L. Christiaen, Z. Kozmik, P. Müller, M. Bronner, R. Krumlauf, and C. Mosimann. A conserved regulatory program initiates lateral plate mesoderm emergence across chordates. Nature Communications, 10(1): 1-15, 2019. ISSN 20411723. doi: 10.1038/s41467-019-11561-7. URL http://dx.doi .org/10.1038/s41467-019-11561-7.

F. Qian, F. Zhen, C. Ong, S. W. Jin, H. M. Soo, D. Y. Stainier, S. Lin, J. Peng, and Z. Wen. Microarray analysis of zebrafish cloche mutant using amplified cDNA and identification of potential downstream target genes. Developmental Dynamics, 233(3):1163-1172, 2005. ISSN 10588388. doi: 10.1002/dvdy.20444. 
A. Quillien, J. C. Moore, M. Shin, A. F. Siekmann, T. Smith, L. Y. Pan, C. B. Moens, M. J. Parsons, and N. D. Lawson. Distinct Notch signaling outputs pattern the developing arterial system. Development, 141(7):1544-1552, 2014. ISSN 1477-9129. doi: Doi10.1242/Dev.099986. URL http://dev.biologists.org/content/ 141/7/1544\\%5Cn\\%3CGotoISI \\%3E://000333184500013\\%5Cnhttp: //dev.biologists.org/content/141/7/1544.full.pdf.

R Core Team. R: A Language and Environment for Statistical Computing. R Foundation for Statistical Computing, Vienna, Austria, 2020. URL https://www.R-project.org/.

J. P. Rasmussen, G. S. Sack, S. M. Martin, and A. Sagasti. Vertebrate Epidermal Cells Are Broad-Specificity Phagocytes That Clear Sensory Axon Debris. The Journal of Neuroscience, 35(2):559-570, jan 2015. ISSN 0270-6474. doi: 10.1523/JNEUROSCI.3613-14.2015. URL http://www . jneurosci.org/lookup/doi/10.1523/JNEUROSCI . 3613-14.2015.

S. Reischauer, O. A. Stone, A. Villasenor, N. Chi, S. W. Jin, M. Martin, M. T. Lee, N. Fukuda, M. Marass, A. Witty, I. Fiddes, T. Kuo, W. S. Chung, S. Salek, R. Lerrigo, J. Alsio, S. Luo, D. Tworus, S. M. Augustine, S. Mucenieks, B. Nystedt, A. J. Giraldez, G. P. Schroth, O. Andersson, and D. Y. Stainier. Cloche is a bHLH-PAS transcription factor that drives haemato-vascular specification. Nature, 535(7611):294-298, 2016. ISSN 14764687. doi: 10.1038/nature18614. URL http://dx.doi.org/10.1038/ nature18614.

P. Rice, I. Longden, and A. Bleasby. EMBOSS: The European Molecular Biology Open Software Suite. Trends in Genetics, 16(6):276-277, jun 2000. ISSN 01689525. doi: 10.1016/S0168-9525(00)02024-2.

L. Robb, I. Lyons, R. Li, L. Hartley, F. Köntgen, R. P. Harvey, D. Metcalf, and C. G. Begley. Absence of yolk sac hematopoiesis from mice with a targeted disruption of the scl gene. Proceedings of the National Academy of Sciences of the United States of America, 92(15):7075-7079, 1995. ISSN 00278424. doi: 10.1073/pnas.92.15.7075.

S. M. Robertson, M. Kennedy, J. M. Shannon, and G. Keller. A transitional stage in the commitment of mesoderm to hematopoiesis requiring the transcription factor SCL/tal-1. Development, 127(11):2447-2459, 2000. ISSN 09501991. 
L. A. Rohde, A. C. Oates, and R. K. Ho. A crucial interaction between embryonic red blood cell progenitors and paraxial mesoderm revealed in spadetail embryos. Developmental Cell, 7(2):251-262, 2004. ISSN 15345807. doi: 10.1016/j.devcel.2004.07.010.

B. L. Roman, V. N. Pham, N. D. Lawson, M. Kulik, S. Childs, A. C. Lekven, D. M. Garrity, R. T. Moon, M. C. Fishman, R. J. Lechleider, and B. M. Weinstein. Disruption of acvrl1 increases endothelial cell number in zebrafish cranial vessels. Development, 129(12):3009-3019, 2002. ISSN 0950-1991. URL https://dev.biologists .org/content/129/12/3009.

A. Rossi, Z. Kontarakis, C. Gerri, H. Nolte, S. Hölper, M. Krüger, and D. Y. R. Stainier. Genetic compensation induced by deleterious mutations but not gene knockdowns. Nature, 524(7564):230-233, aug 2015. ISSN 0028-0836. doi: 10.1038/nature14580. URL http://www.nature.com/ articles/nature14580.

J. M. Rowlinson and M. Gering. Hey2 acts upstream of Notch in hematopoietic stem cell specification in zebrafish embryos. Blood, 116(12):20462056, 2010. ISSN 15280020. doi: 10.1182/blood-2009-11-252635.

L. Ruzicka, D. G. Howe, S. Ramachandran, S. Toro, C. E. Van Slyke, Y. M. Bradford, A. Eagle, D. Fashena, K. Frazer, P. Kalita, P. Mani, R. Martin, S. T. Moxon, H. Paddock, C. Pich, K. Schaper, X. Shao, A. Singer, and M. Westerfield. The Zebrafish Information Network: new support for non-coding genes, richer Gene Ontology annotations and the Alliance of Genome Resources. Nucleic acids research, 47 (D1):D867-D873, 2019. ISSN 1362-4962. doi: 10.1093/nar/gky1090. URL http://www.ncbi.nlm.nih.gov/pubmed/30407545http://www . pubmedcentral.nih.gov/articlerender.fcgi?artid=PMC6323962.

P. Sahai-Hernandez, C. Pouget, O. Svoboda, and D. Traver. Dermomyotomederived endothelial cells migrate to the dorsal aorta to support hematopoietic stem cell emergence. bioRxiv, page 2020.05.14.096305, jan 2020. doi: 10.1101/2020.05.14.096305. URL http://biorxiv.org/content/ early/2020/05/15/2020.05.14.096305. abstract.

M. M. Santoro, G. Pesce, and D. Y. Stainier. Characterization of Vascular Mural Cells During Zebrafish Development. Mechanisms of development, 126(8-9):638-649, 2009. ISSN 0925-4773. doi: 10.1016/j.mod.2009.06.1080. URL http://www.ncbi.nlm.nih.gov/pmc/articles/PMC2732398/. 
J. Schindelin, I. Arganda-Carreras, E. Frise, V. Kaynig, M. Longair, T. Pietzsch, S. Preibisch, C. Rueden, S. Saalfeld, B. Schmid, J.-Y. Tinevez, D. J. White, V. Hartenstein, K. Eliceiri, P. Tomancak, and A. Cardona. Fiji: an open-source platform for biological-image analysis. Nature Methods, 9 (7):676-682, jul 2012. ISSN 1548-7091. doi: 10.1038/nmeth.2019. URL http://www . nature.com/articles/nmeth. 2019.

J. J. Schoenebeck, B. R. Keegan, and D. Yelon. Vessel and Blood Specification Override Cardiac Potential in Anterior Mesoderm. Developmental Cell, 13(2):254-267, 2007. ISSN 15345807. doi: 10.1016/j.devcel.2007.05. 012 .

S. Schulte-Merker, F. J. van Eeden, M. E. Halpern, C. B. Kimmel, and C. Nüsslein-Volhard. no tail (ntl) is the zebrafish homologue of the mouse T (Brachyury) gene. Development (Cambridge, England), 120(4):100915, apr 1994. ISSN 0950-1991. URL http://www.ncbi.nlm.nih.gov/ pubmed/7600949.

J. Schultz, F. Milpetz, P. Bork, and C. P. Ponting. SMART, a simple modular architecture research tool: Identification of signaling domains. Proceedings of the National Academy of Sciences, 95(11):5857-5864, may 1998. ISSN 0027-8424. doi: 10.1073/pnas.95.11.5857. URL http://www.pnas.org/ cgi/doi/10.1073/pnas.95.11.5857.

J. A. Schumacher, J. Bloomekatz, Z. V. Garavito-Aguilar, and D. Yelon. Tal1 regulates the formation of intercellular junctions and the maintenance of identity in the endocardium. Developmental Biology, 383(2):214-226, 2013. ISSN 1095564X. doi: 10.1016/j.ydbio.2013.09.019. URL http: //dx.doi.org/10.1016/j.ydbio.2013.09.019.

F. Shalaby, J. Rossant, T. P. Yamaguchi, M. Gertsenstein, X.-F. Wu, M. L. Breitman, and A. C. Schuh. Failure of blood-island formation and vasculogenesis in Flk-1-deficient mice. Nature, 376(6535):62-66, jul 1995. ISSN 0028-0836. doi: 10.1038/376062a0. URL https ://www . nature.com/ articles/376062a0.pdfhttp://www . nature.com/articles/376062a0.

R. A. Shivdasanl, E. L. Mayer, and S. H. Orkin. Absence of blood formation in mice lacking the T-cell leukaemia oncoprotein tal-1/SCL. Nature, 373 (6513):432-434, 1995. ISSN 00280836. doi: 10.1038/373432a0.

A. F. Siekmann and N. D. Lawson. Notch signalling limits angiogenic cell behaviour in developing zebrafish arteries. Nature, 445(7129):781-784, 2007. ISSN 14764687. doi: 10.1038/nature05577. 
D. D. Spyropoulos, P. N. Pharr, K. R. Lavenburg, P. Jackers, T. S. Papas, M. Ogawa, and D. K. Watson. Hemorrhage, impaired hematopoiesis, and lethality in mouse embryos carrying a targeted disruption of the Fli1 transcription factor. Molecular and cellular biology, 20(15): 5643-52, aug 2000. ISSN 0270-7306. doi: 10.1128/mcb.20.15.5643-5652. 2000. URL http://www.ncbi.nlm.nih.gov/pubmed/10891501http:// www . pubmedcentral . nih.gov/articlerender . fcgi?artid=PMC86032.

D. Y. Stainier, B. M. Weinstein, H. W. Detrich, L. I. Zon, and M. C. Fishman. Cloche, an early acting zebrafish gene, is required by both the endothelial and hematopoietic lineages. Development (Cambridge, England), 121(10): 3141-50, oct 1995. ISSN 0950-1991. doi: 10.5604/01.3001.0010.8250. URL http://www.ncbi.nlm.nih.gov/pubmed/7588049.

D. Y. Stainier, B. Fouquet, J. N. Chen, K. S. Warren, B. M. Weinstein, S. E. Meiler, M. A. P. Mohideen, S. C. Neuhauss, L. Solnica-Krezel, A. F. Schier, F. Zwartkruis, D. L. Stemple, J. Malicki, W. Driever, and M. C. Fishman. Mutations affecting the formation and function of the cardiovascular system in the zebrafish embryo. Development, 123:285-292, 1996. ISSN 09501991. doi: 10.5167/uzh-237.

O. A. Stone and D. Y. Stainier. Paraxial Mesoderm Is the Major Source of Lymphatic Endothelium. Developmental Cell, pages 1-9, 2019. ISSN 15345807. doi: 10.1016/j.devcel.2019.04.034. URL https://linkinghub . elsevier.com/retrieve/pii/S1534580719303314.

F. Strobl, S. Klees, and E. H. K. Stelzer. Light Sheet-based Fluorescence Microscopy of Living or Fixed and Stained Tribolium castaneum Embryos. Journal of visualized experiments : JoVE, (122), 2017. ISSN 1940-087X. doi: 10.3791/55629. URL http://www.ncbi.nlm.nih.gov/pubmed/28518097http://www . pubmedcentral.nih.gov/articlerender.fcgi?artid=PMC5565123.

S. Sumanas, G. Gomez, Y. Zhao, C. Park, K. Choi, and S. Lin. Interplay among Etsrp ER71, Scl, and Alk8 signaling controls endothelial and myeloid cell formation. Blood, 111(9):4500-4510, 2008. ISSN 00064971. doi: 10.1182/blood-2007-09-110569.

S. Sumanas and S. Lin. Ets1-related protein is a key regulator of vasculogenesis in zebrafish. PLoS Biology, 4(1):0060-0069, 2006. ISSN 15457885. doi: 10.1371/journal.pbio.0040010. 
S. Sumanas, T. Jorniak, and S. Lin. Identification of novel vascular endothelial-specific genes by the microarray analysis of the zebrafish cloche mutants. Blood, 106(2):534-541, 2005. ISSN 00064971. doi: 10.1182/blood-2004-12-4653.

M. Takasato and M. H. Little. A strategy for generating kidney organoids: Recapitulating the development in human pluripotent stem cells. Developmental Biology, 420(2):210-220, 2016. ISSN 1095564X. doi: 10.1016/ j.ydbio.2016.08.024. URL http://dx.doi.org/10.1016/j ·ydbio. 2016 . 08.024 .

M. Takeuchi, Y. Fuse, M. Watanabe, C. S. Andrea, M. Takeuchi, H. Nakajima, K. Ohashi, H. Kaneko, M. Kobayashi-Osaki, M. Yamamoto, and M. Kobayashi. LSD1/KDM1A promotes hematopoietic commitment of hemangioblasts through downregulation of Etv2. Proceedings of the $\mathrm{Na}$ tional Academy of Sciences of the United States of America, 112(45): 13922-13927, 2015. ISSN 10916490. doi: 10.1073/pnas.1517326112.

C. Thisse and B. Thisse. High-resolution in situ hybridization to whole-mount zebrafish embryos. Nature Protocols, 3(1):59-69, 2008. ISSN 17542189. doi: 10.1038/nprot.2007.514.

M. A. Thompson, D. G. Ransom, S. J. Pratt, H. MacLennan, M. W. Kieran, H. W. Detrich, B. Vail, T. L. Huber, B. Paw, A. J. Brownlie, A. C. Oates, A. Fritz, M. A. Gates, A. Amores, N. Bahary, W. S. Talbot, H. Her, D. R. Beier, J. H. Postlethwait, and L. I. Zon. The cloche and spadetail genes differentially affect hematopoiesis and vasculogenesis. Developmental Biology, 197(2):248-269, 1998. ISSN 00121606. doi: 10.1006/dbio.1998. 8887.

H. Thorvaldsdóttir, J. T. Robinson, and J. P. Mesirov. Integrative Genomics Viewer (IGV): high-performance genomics data visualization and exploration. Briefings in Bioinformatics, 14(2):178-192, 04 2012. ISSN 14675463. doi: 10.1093/bib/bbs017. URL https://doi.org/10.1093/bib/ bbs017.

V. A. Traag, L. Waltman, and N. J. van Eck. From Louvain to Leiden: guaranteeing well-connected communities. Scientific Reports, 9(1):5233, 2019. ISSN 2045-2322. doi: 10.1038/s41598-019-41695-z. URL https: //doi.org/10.1038/s41598-019-41695-z.

G. Van Rossum and F. L. Drake Jr. Python tutorial. Centrum voor Wiskunde en Informatica Amsterdam, The Netherlands, 1995. 
M. B. Veldman and S. Lin. Etsrp/Etv2 is directly regulated by Foxc1a/b in the zebrafish angioblast. Circulation Research, 110(2):220-229, 2012. ISSN 00097330. doi: 10.1161/CIRCRESAHA.111.251298.

M. B. Veldman, C. Zhao, G. A. Gomez, A. G. Lindgren, H. Huang, H. Yang, S. Yao, B. L. Martin, D. Kimelman, and S. Lin. Transdifferentiation of Fast Skeletal Muscle Into Functional Endothelium in Vivo by Transcription Factor Etv2. PLoS Biology, 11(6), 2013. ISSN 15449173. doi: 10.1371/ journal.pbio.1001590.

K. M. Vogeli, S. W. Jin, G. R. Martin, and D. Y. Stainier. A common progenitor for haematopoietic and endothelial lineages in the zebrafish gastrula. Nature, 443(7109):337-339, 2006. ISSN 14764687. doi: $10.1038 /$ nature05045.

D. E. Wagner, C. Weinreb, Z. M. Collins, J. A. Briggs, S. G. Megason, and A. M. Klein. Single-cell mapping of gene expression landscapes and lineage in the zebrafish embryo. Science, 360(6392):981-987, 2018. ISSN 10959203. doi: $10.1126 /$ science.aar4362.

M. Wang, Z. Zhong, Y. Zhong, W. Zhang, and H. Wang. The Zebrafish Period2 Protein Positively Regulates the Circadian Clock through Mediation of Retinoic Acid Receptor (RAR)-related Orphan Receptor $\alpha$ (Ror $\alpha$ ). Journal of Biological Chemistry, 290(7):4367-4382, feb 2015. ISSN 00219258. doi: 10.1074/jbc.M114.605022. URL https://linkinghub. elsevier.com/retrieve/pii/S0021925819470233.

Q. Wang, Y. Lan, E.-S. Cho, K. M. Maltby, and R. Jiang. Oddskipped related 1 (Odd 1) is an essential regulator of heart and urogenital development. Developmental biology, 288(2):582594, dec 2005. ISSN 0012-1606. doi: 10.1016/j.ydbio.2005.09. 024. URL https://pubmed.ncbi.nlm.nih.gov/16223478https://www . ncbi.nlm.nih.gov/pmc/articles/PMC3869089/.

Y. Wang, L. Pan, C. B. Moens, and B. Appel. Notch3 establishes brain vascular integrity by regulating pericyte number. Development (Cambridge, England), 141(2):307-17, 2014. ISSN 1477-9129. doi: 10.1242/dev. 096107. URL http://www.pubmedcentral.nih.gov/articlerender. fcgi?artid=3879812\\&tool=pmcentrez \\&rendertype=abstract.

R. M. Warga, R. L. Mueller, R. K. Ho, and D. A. Kane. Zebrafish Tbx16 regulates intermediate mesoderm cell fate by attenuating Fgf activity. Developmental Biology, 383(1):75-89, 2013. ISSN 0012-1606. doi: https:// 
doi.org/10.1016/j.ydbio.2013.08.018. URL http://www.sciencedirect. com/science/article/pii/S0012160613004430.

A. J. Warren, W. H. Colledge, M. B. Carlton, M. J. Evans, A. J. Smith, and T. H. Rabbitts. The Oncogenic Cysteine-rich LIM domain protein Rbtn2 is essential for erythroid development. Cell, 78(1):45-57, 1994. ISSN 00928674. doi: 10.1016/0092-8674(94)90571-1.

P. Wasteson, B. R. Johansson, T. Jukkola, S. Breuer, L. M. Akydsurek, J. Partanen, and P. Lindahl. Developmental origin of smooth muscle cells in the descending aorta in mice. Development, 135(10):1823-1832, 2008. ISSN 09501991. doi: 10.1242/dev.020958.

Wei Weng, Hiroki Nagai, Sofiane Hamidi, and Guojun Sheng. NPAS4L is involved in avian hemangioblast specification. Haematologica, 105(11 SE - Letters to the Editor):2647-2650, feb 2020. doi: 10.3324/haematol.2019. 239434. URL https://haematologica.org/article/view/9651.

O. Weiss, R. Kaufman, N. Michaeli, and A. Inbal. Abnormal vasculature interferes with optic fissure closure in lmo2 mutant zebrafish embryos. Developmental Biology, 369(2):191-198, 2012. ISSN 1095564X. doi: 10.1016/j.ydbio.2012.06.029. URL http://dx.doi.org/10.1016/j . ydbio.2012.06.029.

W. A. Wierson, J. M. Welker, M. P. Almeida, C. M. Mann, D. A. Webster, M. E. Torrie, T. J. Weiss, S. Kambakam, M. K. Vollbrecht, M. Lan, K. C. McKeighan, J. Levey, Z. Ming, A. Wehmeier, C. S. Mikelson, J. A. Haltom, K. M. Kwan, C. B. Chien, D. Balciunas, S. C. Ekker, K. J. Clark, B. R. Webber, B. S. Moriarity, S. L. Solin, D. F. Carlson, D. L. Dobbs, M. McGrail, and J. Essner. Efficient targeted integration directed by short homology in zebrafish and mammalian cells. eLife, 9:1-25, 2020. ISSN 2050084X. doi: 10.7554/eLife.53968.

D. M. Wiley, J. D. Kim, J. Hao, C. C. Hong, V. L. Bautch, and S. W. Jin. Distinct signalling pathways regulate sprouting angiogenesis from the dorsal aorta and the axial vein. Nature Cell Biology, 13(6):686-693, 2011. ISSN 14657392. doi: 10.1038/ncb2232. URL http://dx.doi.org/10. 1038/ncb2232.

J. Wilting, M. Papoutsi, M. Schneider, and B. Christ. The lymphatic endothelium of the avian wing is of somitic origin. Developmental Dynamics: An Official Publication of the American Association of Anatomists, 217(3):271-278, mar 2000. ISSN 1058-8388. doi: 10.1002/ 
(SICI)1097-0177(200003)217:3〈271::AID-DVDY5>3.0.CO;2-2. URL http: //www.ncbi.nlm.nih.gov/pubmed/10741421.

F. A. Wolf, P. Angerer, and F. J. Theis. SCANPY: large-scale single-cell gene expression data analysis. Genome Biology, 19(1):15, dec 2018. ISSN 1474760X. doi: 10.1186/s13059-017-1382-0. URL https://genomebiology . biomedcentral.com/articles/10.1186/s13059-017-1382-0.

F. A. Wolf, F. K. Hamey, M. Plass, J. Solana, J. S. Dahlin, B. Göttgens, N. Rajewsky, L. Simon, and F. J. Theis. PAGA: graph abstraction reconciles clustering with trajectory inference through a topology preserving map of single cells. Genome Biology, 20(1):59, 2019. ISSN 1474760X. doi: 10.1186/s13059-019-1663-x. URL https ://doi.org/10.1186/ s13059-019-1663-x.

P. Xu, G. Zhu, Y. Wang, J. Sun, X. Liu, Y.-G. Chen, and A. Meng. Maternal Eomesodermin regulates zygotic nodal gene expression for mesendoderm induction in zebrafish embryos. Journal of molecular cell biology, 6(4): 272-85, aug 2014. ISSN 1759-4685. doi: 10.1093/jmcb/mju028. URL http://www.ncbi.nlm.nih.gov/pubmed/24924767.

P. Xu, B. Balczerski, A. Ciozda, K. Louie, V. Oralova, A. Huysseune, and J. G. Crump. Fox proteins are modular competency factors for facial cartilage and tooth specification. Development, 145(12), 2018. ISSN 09501991. doi: 10.1242/dev.165498. URL https://dev.biologists.org/ content/145/12/dev165498.

K. Yano, D. Gale, S. Massberg, P. K. Cheruvu, R. Monahan-Earley, E. S. Morgan, D. Haig, U. H. von Andrian, A. M. Dvorak, and W. C. Aird. Phenotypic heterogeneity is an evolutionarily conserved feature of the endothelium. Blood, 109(2):613-615, jan 2007. ISSN 0006-4971. doi: 10.1182/blood-2006-05-026401. URL https://ashpublications.org/blood/article/109/2/613/23515/ Phenotypic-heterogeneity-is-an-evolutionarily.

A. D. Yates, P. Achuthan, W. Akanni, J. Allen, J. Allen, J. Alvarez-Jarreta, M. R. Amode, I. M. Armean, A. G. Azov, R. Bennett, J. Bhai, K. Billis, S. Boddu, J. C. Marugán, C. Cummins, C. Davidson, K. Dodiya, R. Fatima, A. Gall, C. G. Giron, L. Gil, T. Grego, L. Haggerty, E. Haskell, T. Hourlier, O. G. Izuogu, S. H. Janacek, T. Juettemann, M. Kay, I. Lavidas, T. Le, D. Lemos, J. G. Martinez, T. Maurel, M. McDowall, A. McMahon, S. Mohanan, B. Moore, M. Nuhn, D. N. Oheh, A. Parker, A. Parton, M. Patricio, M. P. Sakthivel, A. I. Abdul Salam, B. M. 
Schmitt, H. Schuilenburg, D. Sheppard, M. Sycheva, M. Szuba, K. Taylor, A. Thormann, G. Threadgold, A. Vullo, B. Walts, A. Winterbottom, A. Zadissa, M. Chakiachvili, B. Flint, A. Frankish, S. E. Hunt, G. IIsley, M. Kostadima, N. Langridge, J. E. Loveland, F. J. Martin, J. Morales, J. M. Mudge, M. Muffato, E. Perry, M. Ruffier, S. J. Trevanion, F. Cunningham, K. L. Howe, D. R. Zerbino, and P. Flicek. Ensembl 2020. Nucleic Acids Research, nov 2019. ISSN 0305-1048. doi: 10.1093/nar/ gkz966. URL https://academic.oup.com/nar/advance-article/doi/ 10.1093/nar/gkz966/5613682.

J. Ye, G. Coulouris, I. Zaretskaya, I. Cutcutache, S. Rozen, and T. L. Madden. Primer-BLAST: A tool to design target-specific primers for polymerase chain reaction. BMC Bioinformatics, 13(1): 134, dec 2012. ISSN 1471-2105. doi: 10.1186/1471-2105-13-134. URL https://bmcbioinformatics.biomedcentral.com/articles/10. 1186/1471-2105-13-134.

Y. Yue, M. Jiang, L. He, Z. Zhang, Q. Zhang, C. Gu, M. Liu, N. Li, and Q. Zhao. The transcription factor Foxcla in zebrafish directly regulates expression of nkx2.5, encoding a transcriptional regulator of cardiac progenitor cells. Journal of Biological Chemistry, 293(2):638-650, jan 2018. ISSN 00219258. doi: 10.1074/jbc.RA117.000414. URL https: //linkinghub.elsevier.com/retrieve/pii/S0021925820404442.

X. Y. Zhang and A. R. Rodaway. SCL-GFP transgenic zebrafish: In vivo imaging of blood and endothelial development and identification of the initial site of definitive hematopoiesis. Developmental Biology, 307(2):179194, 2007. ISSN 00121606. doi: 10.1016/j.ydbio.2007.04.002. 